\title{
Titchmarsh-Sims-Weyl theory for complex Hamiltonian systems of arbitrary order
}

\author{
Zur Erlangung des akademischen \\ Grades eines \\ DOKTORS DER NATURWISSENSCHAFTEN \\ der Fakultät für Mathematik der \\ Universität Karlsruhe vorgelegte \\ DISSERTATION \\ von \\ Dipl.-Math. Marco Muzzulini \\ aus Pforzheim
}

Tag der mündlichen Prüfung: 14.11.2007

Referent:

Prof. Dr. Michael Plum

Korreferent:

Prof. Dr. Don B. Hinton

Korreferent:

HDoz. Dr. Peer C. Kunstmann 


\section{Contents}

1 Introduction $\quad 4$

2 Weyl-circle analysis $\quad 12$

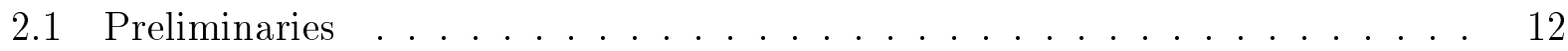

2.2 Generalized Weyl-circles . . . . . . . . . . . . . . 16

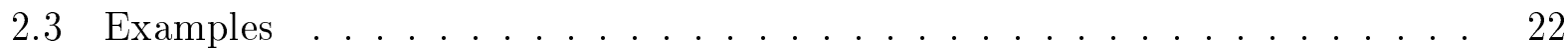

$\begin{array}{lll}3 & \text { The adjoint problem } & 31\end{array}$

3.1 Some linear algebraic statements . . . . . . . . . . . . . . 31

3.2 A suitable pair $(\widehat{V}, \widehat{E})$ for the adjoint Problem $\ldots \ldots \ldots \ldots$

3.3 Weyl-Sims circles in the adjoint setting . . . . . . . . . . . . 42

3.4 Smoothness of $\lambda \mapsto Y_{\lambda} \ldots \ldots \ldots \ldots \ldots \ldots$

$\begin{array}{lll}4 & \text { A differential operator } & 47\end{array}$

4.1 Hilbert spaces . . . . . . . . . . . . . . . . 47

4.2 The $M$-function . . . . . . . . . . . . . . . 50

4.3 The resolvent operator . . . . . . . . . . . . . . . 55

4.4 Definition and properties of the differential operator . . . . . . . . . . 59

4.5 The maximal operator and deficiency indices . . . . . . . . . . . 67 
5.1 Dimensions of the solution spaces . . . . . . . . . . . 73

5.2 Connection between the limit sets and the deficiency index of the maximal

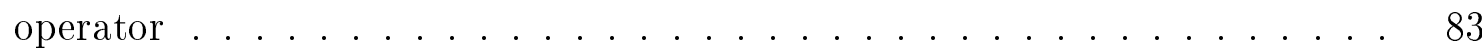

5.3 Properties of the M-function . . . . . . . . . . . . . 84

5.4 Further spectral estimates . . . . . . . . . . . . . . 87

5.4.1 Restriction of the problem to $[\tilde{a}, b) \subseteq[a, b) \ldots \ldots . \ldots 87$

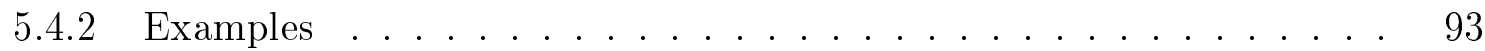

5.4 .3 Variation of the admissible matrix $V \ldots \ldots . \ldots . \ldots 94$

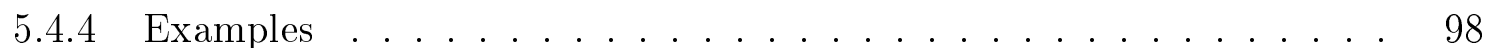

6 Final considerations $\quad 104$

6.1 On the choice of an admissible $V \ldots \ldots \ldots \ldots \ldots$

6.2 On the wedges $\Lambda_{V}$ and the convex sets $W_{V} \ldots \ldots \ldots \ldots$

6.3 An augmentation method . . . . . . . . . . . . 110

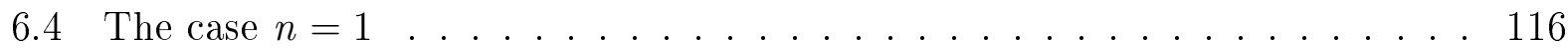




\title{
Chapter 1
}

\section{Introduction}

\author{
Abstract. \\ We consider the formal Hamiltonian system

$$
J Y^{\prime}=(\lambda A+B) Y
$$

where $J J=-I_{n}, A$ and $B$ are $\mathbb{C}^{n, n}$-valued functions on $[a, b), A$ is pointwise self-adjoint and positive semidefinite, but $B$ need not be self-adjoint. The order $n$ is arbitrary.

Our aim is to construct closed differential operators with non-empty resolvent set, which realize the formal system within an appropriate Hilbert space. Therefor we first construct the corresponding resolvent operator via a matrix-valued Titchmarsh-Sims-Weyl type $M$ function.

It turns out that the analysis of the adjoint problem is essential for the theory. Fortunately this analysis does not generate any further assumptions.

Besides the norm generated by $A$, another Sobolev-type norm appears which seems to be some kind of natural for the problem. The geometry of the generalised Weyl limit-sets depend strongly on the number of linearly independent solutions of (1.1), of which this second norm is finite. The limit-point and limit-circle classification is transferred, whereas the nature of this problem yields several cases to be distinguished.

Furthermore a connection between the geometry of the limit-sets and the deficiency indices is presented.

1. The classical Titchmarsh-Weyl theory (see [23], [20], [8, Chapter XIII]) analyses the Sturm-Liouville eigenvalue equation

$$
-y^{\prime \prime}+q y=\lambda y
$$

on $[a, b)$, where $q:[a, b) \rightarrow \mathbb{R}$ is continuous and $b=\infty$ is allowed, with the following results: 
For $\alpha \in[0,2 \pi)$ let $\theta_{\lambda}, \phi_{\lambda}:[a, b) \rightarrow \mathbb{R}$ the unique solutions of (1.2) with initial values

$$
\begin{aligned}
\theta_{\lambda}(a) & =\cos \alpha, & \theta_{\lambda}^{\prime}(a) & =\sin \alpha, \\
\phi_{\lambda}(a) & =\sin \alpha, & \phi_{\lambda}^{\prime}(a) & =-\cos \alpha .
\end{aligned}
$$

For $c \in(a, b)$ and $\lambda$ within the upper (or the lower) halfplane of $\mathbb{C}$, the set of all $l \in \mathbb{C}$ such that

$$
\int_{a}^{c}\left|\theta_{\lambda}+l \phi_{\lambda}\right|^{2} d x \leq-\frac{\operatorname{Im} l}{\operatorname{Im} \lambda}
$$

is a closed disc in $\mathbb{C}$. Let us call it $D_{\lambda}(c)$.

Equation (1.3) shows two things: For $[a, b) \ni \widetilde{c} \geq c$ we have $D_{\lambda}(\widetilde{c}) \subseteq D_{\lambda}(c)$, so the discs are nested. Hence

$$
D_{\lambda}(b):=\bigcap_{c \in[a, b)} D_{\lambda}(c)
$$

is not empty.

Furthermore, for $l \in D_{\lambda}(b)$, the function $\theta_{\lambda}+l \phi_{\lambda}$ is square integrable over $(a, b)$.

There are two geometric possibilities for $D_{\lambda}(b)$ :

It may consist of one point. This is called the limit-point case. The element $l$ of $D_{\lambda}(b)$ is called the limit-point. In this case there exists no further linearly independent solution of (1.2) in $L^{2}(a, b)$ besides $\theta_{\lambda}+\phi_{\lambda} l$.

Alternatively $D_{\lambda}(b)$ could be a disc, which is called the limit-circle case. The boundary of $D_{\lambda}(b)$ is called the limit-circle. In this case every solution of $(1.2)$ is in $L^{2}(a, b)$.

The case is independent of the choice of $\lambda \in \mathbb{C} \backslash \mathbb{R}$.

For fixed $c \in(a, b)$ and for $\beta \in[0,2 \pi)$, one can choose $l \in \partial D_{\lambda}(c)$ by the modified Möbius transformation

$$
l_{\lambda}(c):=\frac{\theta_{\lambda}(c) \cot \beta+\theta_{\lambda}^{\prime}(c)}{\phi_{\lambda}(c) \cot \beta+\phi_{\lambda}^{\prime}(c)} .
$$

(The mapping $\beta \mapsto l_{\lambda}(c)$ maps $[0,2 \pi)$ onto $\partial D_{\lambda}(c)$.) In each case $l_{\lambda}$ converges to some point $m(\lambda) \in \partial D_{\lambda}(b)$ as $c$ tends to $b$, when $\beta$ is kept fixed.

The resulting function $m$ is called the Titchmarsh-Weyl $m$-coefficient. $m$ is holomorphic on the upper halfplane of $\mathbb{C}$, even meromorphic on $\mathbb{C}$ in the limit-circle case.

We choose some $\beta \in[0,2 \pi)$ and therewith some function $m$. (In the limit-point case there is only one $m$-function, independent of $\beta$.)

We set $\psi_{\lambda}:=\theta_{\lambda}+m(\lambda) \phi_{\lambda} \in L^{2}(a, b)$. The function

$$
G_{\lambda}:[a, b)^{2} \rightarrow \mathbb{R}, G_{\lambda}(x, y):= \begin{cases}\psi_{\lambda}(y) \phi_{\lambda}(x) & \text { for } x \leq y, \\ \psi_{\lambda}(x) \phi_{\lambda}(y) & \text { for } y \leq x,\end{cases}
$$

is a Green's function for (1.2). This means that for $f \in L^{2}(a, b)$, the function $g$ defined by

$$
g(x):=\int_{a}^{b} G_{\lambda}(x, y) f(y) d y
$$


solves $-g^{\prime \prime}+(q-\lambda) g=f$, where $g, g^{\prime} \in A C_{\text {loc }}$. Let $\mu$ in the upper halfplane. For the operator $L$, defined by

$$
\begin{aligned}
D(L)=\left\{h \in L^{2}(a, b) \cap A C_{\mathrm{loc}}[a, b):\right. & h^{\prime} \in A C_{\mathrm{loc}}[a, b),-h^{\prime \prime}+q h \in L^{2}(a, b), \\
& \left.h(a) \cos \alpha+h^{\prime}(a) \sin \alpha=0, \text { and } \lim _{x \rightarrow b} \psi_{\mu}(x) h(x)=0\right\}, \\
L h=-h^{\prime \prime}+q h \text { for } h \in D(L) &
\end{aligned}
$$

we have furthermore $g \in D(L)$ with $(L-\lambda I) g=f$. Hence (1.4) defines the resolvent of $L$ at $\lambda$. The definition of $L$ does not depend on the actual choice of $\mu$.

It is shown that $L$ is self-adjoint. To achieve this, the choice of $m$ on the boundary of $D_{\lambda}(b)$ is crucial.

In the limit-point case, the boundary condition at $b$ is redundant.

2. The theory was generalized to the equation

$$
-\left(p y^{\prime}\right)^{\prime}+q y=\lambda w y
$$

with $\frac{1}{p}, q, w \in L_{\mathrm{loc}}^{1}[a, b), w \geq 0$ on $[a, b)$ and $w>0$ on a set of positive measure, further to the higher order formally symmetric scalar differential equation

$$
\sum_{j=0}^{h}(-1)^{j}\left(p_{j} y^{(j)}\right)^{(j)}+\frac{1}{2} i \sum_{j=0}^{k-1}(-1)^{j}\left(\left(q_{j} y^{(j)}\right)^{(j+1)}+\left(q_{j} y^{(j+1)}\right)^{(j)}\right)=\lambda w y
$$

with real coefficient functions $p_{j} \in C^{j}(a, b), q_{j} \in C^{j+1}(a, b)$. See [9, section 3], [10], and for an historical survey, [11].

Here a larger set of linearly independent solutions $\theta_{\lambda, j}$ and $\phi_{\lambda, j}$ is required, fulfilling

$$
\left[\theta_{\lambda, j}, \theta_{\lambda, r}\right](a)=\left[\phi_{\lambda, j}, \phi_{\lambda, r}\right](a)=0 \text { and }\left[\phi_{\lambda, j}, \theta_{\lambda, r}\right](a)=\delta_{j r}
$$

for some symplectic product $[\cdot, \cdot]$, except, if the highest derivative in (1.7) is odd; then the requirement (1.8) becomes a little more involved. Furthermore the function $m$ is matrixvalued.

More cases in between limit-point and limit-circle are possible.

It should be remarked that in this setting, the symmetric operator, created by the theory, is not always self-adjoint. Some criteria even yield that no self-adjoint representation of (1.7) exists (cf. $[17, \S 14,17])$.

For a more general version of (1.7) with $L_{\text {loc }}^{1}$ coefficient functions see [18, section 15 and 16], [21] and the survey paper [24].

3. Equation (1.7) may be transformed into a first-order system of differential equations. So it is a natural question, if the Titchmarsh-Weyl theory can be transferred to systems. Important results are for example in [1], [13], [14] and [16]. 
For this thesis, the most important paper for formally self-adjoint systems is [12], where Hinton and Schneider have investigated the Hamiltonian System

$$
J Y^{\prime}=(\lambda A+B) Y
$$

with matrix-valued, self-adjoint, locally integrable functions $A$ and $B$. $A$ is positive semidefinite on $[a, b)$. Furthermore $i J$ is unitary and self-adjoint.

$\Theta_{\lambda}$ and $\Phi_{\lambda}$ are matrix-valued functions, such that $\left(\Theta_{\lambda} \mid \Phi_{\lambda}\right)$ is a solution of (1.9), where the number of columns of these functions equals the dimensions of the eigenspaces of $J$ to the eigenvalues plus and minus $i$, respectively. These numbers interchange when $\operatorname{Im}(\lambda)$ changes its sign. So does the number of rows and columns of the $M$-function (see below).

The property, that

$$
\left(\Theta_{\lambda} \mid \Phi_{\lambda}\right)(a) \text { is unitary and of a special shape, }
$$

takes the place of (1.8).

With a limit-circle assumption, the existence of a unique (in general non-square) matrixvalued $M$-function is shown, such that every column of $\Theta_{\lambda}+\Phi_{\lambda} M(\lambda)$ is in $L_{A}^{2}(a, b)$. This means

$$
\int_{a}^{b}\left(\Theta_{\lambda}+\Phi_{\lambda} M(\lambda)\right)^{*} A\left(\Theta_{\lambda}+\Phi_{\lambda} M(\lambda)\right) d x<\infty .
$$

The $M$-function is holomorphic on $\mathbb{C} \backslash \mathbb{R}$.

Using the $M$-function, a resolvent is constructed, and therewith a closed operator $\mathbf{A}$ and its adjoint $\mathbf{B}$ within a suitable Hilbert space. The upper halfplane is in the resolvent set of $\mathbf{A}$, the lower halfplane in the resolvent set of $\mathbf{B}$. There are further statements on the spectrum of $\mathbf{A}$ and $\mathbf{B}$ within the respective other halfplane.

4. Another generalization of (1.2) is to allow $q$ to be complex. Sims has examined this problem in [19]. He required, besides continuity of $q$, that the imaginary part of $q$ is restricted to one sign. For $\lambda \in \mathbb{C} \backslash \mathbb{R}$, where $\operatorname{Im}(\lambda)$ is of the opposite sign, the two cases (limit-point or limit-circle) appear. Again they depend on $q$ alone, not on the choice of $\lambda$.

Yet now the limit-point case does not imply that only one solution of (1.2) is square integrable.

In both cases there exist analytic $m$-functions. In the limit-circle case, for fixed $\lambda$ and any point $z$ on the corresponding limit-circle, there exists an $m$-function with $m(\lambda)=z$.

Furthermore, if there are two square integrable linearly independent solutions of (1.2), then any $m$-function is meromorphic on $\mathbb{C}$.

5. In [6], equation (1.6) is considered, again with $\frac{1}{p}, q, w \in L_{\mathrm{loc}}^{1}[a, b), w \geq 0$ on $[a, b)$ and $w>0$ on a set of positive measure. Now $p$ and $q$ are allowed to have complex values.

To get the complex quantities $p$ and $q$ under control, some adequate rotation parameter $\eta$ 
and some shift parameter $K$ are introduced. The additional requirement that, for every positive $r$ and every $x \in[a, b)$,

$$
\boldsymbol{\operatorname { R e }}\left(\left(r p(x)+\frac{q(x)}{w(x)}-K\right) e^{i \eta}\right) \geq 0
$$

is now necessary to transfer the Titchmarsh-Sims-Weyl theory.

$\lambda$ is chosen in the rotated and shifted halfplane

$$
\Lambda_{\eta, K}:=K+\left\{\nu \in \mathbb{C}: \operatorname{Re}\left(\nu e^{i \eta}\right)<0\right\}
$$

Besides the weighted Hilbert space $L_{w}^{2}$ with norm $\left(\int_{a}^{b} w|f|^{2} d x\right)^{-1 / 2}$ a second Sobolev-type norm was introduced, which dominates the first norm. Therewith the limit-point case with yet two linearly independent solutions in $L_{w}^{2}$ could be explained:

The limit-point case appears if and only if there is only one solution of (1.6) of which the second (Sobolev-type) norm is finite.

An $m$-function is constructed, analytic in $\Lambda_{\eta, K}$ and, if all solutions of (1.6) are in $L_{w}^{2}$, meromorphic on $\mathbb{C}$.

Therewith a closed operator $L$ is constructed. $\Lambda_{\eta, K}$ is a subset of the resolvent set of $L$. Varying the constant $K$, some other rotation parameter $\eta$ may be possible.

Also further choices of $(K, \eta)$ may be possible, if (1.11) should only hold for all $x \in[\tilde{a}, b)$ with some $\tilde{a} \in(a, b)$.

The investigation of these further choices improves the estimations of the spectrum of $L$.

6. This thesis mainly bases on the paper [5] by Brown, Evans and Plum.

Therein the Hamiltonian system (1.9) is considered with $J=\left(\begin{array}{cc}0 & -I_{n} \\ I_{n} & 0\end{array}\right)$ and locally integrable functions $A, B:[a, b) \rightarrow \mathbb{C}^{2 n, 2 n}$, where $A$ is self-adjoint and positive semidefinite on $[a, b)$. Yet $B$ need not be self-adjoint.

Additional assumptions on $A$ and $B$ are made.

The place of $(\eta, K)$ in [6] is taken by some adequate matrix $\mathcal{U}_{2 n}$ and again some constant $k$. In the following, for any square matrix $X$ we use $\operatorname{Re} X$ for the self-adjoint matrix $\frac{1}{2}\left(X+X^{*}\right)$. The aforementioned additional assumptions yield that

$$
C_{k}:=\operatorname{Re}\left(\mathcal{U}_{2 n}(k A+B)\right)
$$

is positive semidefinite on $[a, b)$, and that

$$
\begin{aligned}
\Lambda\left(k, \mathcal{U}_{2 n}\right):=k+\{\lambda \in \mathbb{C}: & \operatorname{Re}\left(\lambda \mathcal{U}_{2 n} A\right)-\delta \mathcal{U}_{2 n} A \mathcal{U}_{2 n}^{*} \\
& \text { is positive semidefinite on }[a, b) \text { for some } \delta>0\}
\end{aligned}
$$

is not empty.

$\lambda$ is now chosen in $\Lambda\left(k, \mathcal{U}_{2 n}\right)$. Again $\Theta_{\lambda}$ and $\Phi_{\lambda}$ are $\mathbb{C}^{2 n, n}$ solutions of (1.9), with initial 
condition $\left(\Theta_{\lambda} \mid \Phi_{\lambda}\right)(a)=E$ for some unitary matrix $E$.

Furthermore the adjoint problem

$$
J Z^{\prime}=\left(\bar{\lambda} A+B^{*}\right) Z
$$

is considered, with $\mathbb{C}^{2 n, n}$ solutions $\eta_{\lambda}$ and $\chi_{\lambda}$, such that $\left(\eta_{\lambda} \mid \chi_{\lambda}\right)(a)=\widehat{E}$, for a certain matrix $\widehat{E}$. The new version of (1.8) reads

$$
[E, \widehat{E}]=\widehat{E}^{*} J E=\left(\begin{array}{cc}
0 & -I_{n} \\
I_{n} & 0
\end{array}\right)
$$

The Weyl-Sims-Titchmarsh sets, corresponding to the circular disks, are defined by

$$
D_{x}(\lambda):=\left\{l \in \mathbb{C}^{n, n}:\left(\Theta_{\lambda}+\Phi_{\lambda} l\right)^{*} \mathcal{U}_{2 n} J\left(\Theta_{\lambda}+\Phi_{\lambda} l\right)(x) \text { is negative semidefinite }\right\} .
$$

It is shown that these sets are non-empty, closed, and eventually nested. The geometry of the limit-set correlates to the number of independent solutions $y$ of (1.9) such that

$$
\|y\|_{C_{\lambda}}^{2}:=\int_{a}^{b} y^{*} C_{\lambda} y d x
$$

is finite, respectively to the corresponding quantity of the adjoint problem (which need not be the same). $\|\cdot\|_{C_{k}}$ and the respective norm for the adjoint problem correspond to the Sobolev-type norm in [6] that determines the limit-point or the limit-circle case.

An $M$-function is constructed, holomorphic at least in $\Lambda\left(k, \mathcal{U}_{2 n}\right)$, furthermore, with an important additional assumption, a resolvent. An operator $L$ for (1.9) is defined via boundary conditions, while the just mentioned assumption yields well-definedness. The corresponding operator for (1.12) is adjoint to $L . \Lambda\left(k, \mathcal{U}_{2 n}\right)$ is a subset of the resolvent set of $L$.

7. This thesis is mainly a generalization of [5] from the even to the arbitrary order. Furthermore it is partially a generalization of [12] to the non self-adjoint case. Some results therein depend on $B$ being self-adjoint and hence could not be achieved here.

In chapter 2 the setting and the fundamental requirements are stated:

(1.9) is considered with locally integrable functions $A, B:[a, b) \rightarrow \mathbb{C}^{n, n}$, where $A$ is selfadjoint and positive semidefinite on $[a, b)$. $i J$ is unitary and self-adjoint.

The existence of some self-adjoint matrix $V$ is assumed (which takes the role of $\mathcal{U}_{2 n} J$ in [5]), such that

$$
C_{k, V}:=-\boldsymbol{R e}(V J(k A+B))
$$

is positive semidefinite a.e. on $[a, b)$ for some $k \in \mathbb{C}$ (which, in contrast to [5], is allowed to vary here), and the set

$$
\Lambda_{V}:=\{\lambda \in \mathbb{C}:-\boldsymbol{R e}(\lambda V J A)-\delta A \text { is positive semidefinite a.e. on }[a, b) \text { for some } \delta>0\}
$$


is not empty.

For a fixed $V$ like this, the number of its positive and negative eigenvalues $\left(n^{+}\right.$and $\left.n^{-}\right)$determines the number of rows of $\Theta_{\lambda}$ and $\Phi_{\lambda}$, and also the number of rows and columns of the $M$-function.

In this non-self-adjoint setting $E=\left(\Theta_{\lambda}(a) \mid \Phi_{\lambda}(a)\right)$ need not be unitary. To omit this requirement is crucial in one proof and useful for an example.

The theory follows the footsteps of [5] to construct the generalized Weyl-circles, now as subsets of $\mathbb{C}^{n^{+}, n^{-}}$.

In chapter 3, the adjoint problem is considered. Using linear algebraic theory, it is shown that no further assumption is necessary for the existence of some corresponding self-adjoint matrix $\widehat{V}$. The constants $n^{+}$and $n^{-}$exchange their parts in the adjoint setting.

In chapter 4 an appropriate $M$-function is constructed. Just one further assumption is required to obtain the corresponding resolvents and operators $L$, respectively $\widehat{L}$ for the adjoint problem. $\widehat{L}$ is adjoint to $L$. There are $n^{-}$, respectively $n^{+}$, boundary conditions at $a$ in the domains of definition of $L$, respectively of $\widehat{L}$.

A connection of the shape of the limit-set to the deficiency indices of the maximal operator (cp. [17, §14]) closes this chapter.

In chapter 5 the classification into limit-circle and limit-point by the geometrical shape of the Weyl-limit-set is transferred. The nature of (1.9) yields more essentially different possibilities, whence more cases are established. Also the number of linearly independant solutions of (1.9), respectively of (1.12), that are in $L_{A}^{2}(a, b)$ was used for further case distinction.

Further investigations of the $M$-function and of the number of linearly independent solutions that are in $L_{A}^{2}$, respectively in $L_{C_{\lambda}}^{2}$, have been transferred from [5] and supplemented.

By restricting the problem to $[\tilde{a}, b)$ for some $\tilde{a} \in(a, b)$, the spectral estimates are improved. This result corresponds to the ansatz in [6] of posing assumptions on $(K, \eta)$ only on the interval $[\tilde{a}, b)$.

Furthermore the variation of the self-adjoint matrix $V$ yields better spectral estimates under some strict limit-point condition.

In chapter 6 , finally, some linear algebra theory provides an insight into various possibilities of choosing parameters (like $V$ ). An augmentation method, which augments the problem to an equivalent problem of higher order yields further miscellaneous results.

Since this thesis is based on [5], some statements are transferred. To point this out, the corresponding statements are marked as follows:

"Statement. ${ }^{* 1 "}$ means the statement and the proof have been essentially transferred from $[5]$.

"Statement. ${ }^{*} "$ means the statement has been transferred from [5]. The proof has partially been varied.

"Statement.. ${ }^{*}$ " means the statement and the proof have been partially transferred from [5]. "Statement.*4" means little parts of the statement and the proof have been transferred from [5]. 


\section{Acknowledgments.}

The author is grateful

to Michael Plum for very helpful discussions and remarks and for his accurate refereeing, to Don Hinton for acting as external referee, for the idea of a connection between the geometry of the Weyl limit-set and the deficiency index, and for further assistance, to Peer Kunstmann for his support and for acting as referee, to Malcolm Brown for the idea of this thesis, to Des Evans for encouraging discussions, to Stefan Kühnlein for the proof of the important Lemma 3.1.1, finally to Ludwig Elsner, Peer Kunstmann, Christoph Schmoeger and Gerd Herzog for their support. 


\section{Chapter 2}

\section{Weyl-circle analysis}

In this chapter we introduce the general settings of the problem, we are concerned with. We define the generalisations of the Weyl-circles as subsets of $\mathbb{C}^{n^{+}, n^{-}}$for some $n^{+}, n^{-} \in \mathbb{N}_{0}$. Finally, we introduce scalar equations that lead to Hamiltonian systems as an example for the theory of this thesis.

\section{$2.1 \quad$ Preliminaries}

We are concerned with the Hamiltonian system

$$
J y_{\lambda}^{\prime}(x)=(\lambda A(x)+B(x)) y_{\lambda}(x)
$$

with $J, A(x)$ and $B(x) \in \mathbb{C}^{n, n}$ for $x \in[a, b) . \lambda \in \mathbb{C}$ is the spectral parameter.

We assume that

(i) $A(x)$ is self-adjoint and positive semidefinite for a.e. $x \in[a, b), A \neq 0$ on a set of positive measure,

(ii) $J$ is unitary and $i J$ is self-adjoint,

(iii) $A$ and $B$ are locally integrable on $[a, b)$.

Our aim is to construct closed differential operators with non-empty resolvent set, realizing the formal equation (2.1), via a matrix-valued M-function, and to give estimates for their spectra. 
In our approach we need a fundamental matrix $Y$ for the differential equation (2.1) with initial conditions at $a$. Not every initial condition is suitable for our approach. Further there is no canonical size for the M-function. This question is equivalent to where the fundamental matrix should be split into two sub-matrices

$$
Y=(\Theta \mid \Phi)
$$

In [5], where the even-order case is examined, $\Phi$ and $\Theta$ have the same size. In our more general approach $n$ is an arbitrary natural number. Also if $n$ is even we do not only consider the "canonical" splitting into two equal parts. Later we will see that sometimes there is no choice of how $Y$ can be split except exchanging the sizes of $\Phi$ and $\Theta$, such that the following theory will lead to a result. Yet for every successful choice there will occur an own operator realization of (2.1) with an own number of boundary conditions at $a$.

Lemma 2.1.1. Equivalent to assumption (iii) is each of the following statements:

$\left(i i i^{\prime}\right):$ For $\lambda \in \mathbb{C}, \xi \in \mathbb{C}^{n}, c \in[a, b)$, there is exactly one solution y of $(2.1)$ in $A C_{\text {loc }}[a, b)$ with $y(c)=\xi$.

$\left(i i i^{\prime \prime}\right):$ For $\lambda \in \mathbb{C}, \Xi \in \mathbb{C}^{n, n}$, there is exactly one solution $Y$ of $(2.1)$ in $A C_{\text {loc }}[a, b)$ with $Y(a)=\Xi$; If $\Xi$ is regular then $Y(c)$ is regular for all $c \in[a, b)$.

Proof. In $\left[22, \S 10\right.$, Theorem XVIII], the implication $(i i i) \Rightarrow\left(i i i^{\prime}\right)$ on $[c, b)$ is shown. On $[a, c]$ we substitute $\tilde{x}:=a+c-x$ and use the same Theorem again.

If $(\text { iii })^{\prime}$ holds, for $\Xi \in \mathbb{C}^{n, n}$ there is exactly one solution $Y$ of $(2.1)$ with $Y(a)=\Xi$.

Let $c \in[a, b)$ and $\xi \in \mathbb{C}^{n}$ with $Y(c) \xi=0$. Thus $Y \xi$ solves $(2.1)$ and $(Y \xi)(c)=0$. With the assumed uniqueness, $(\text { iii })^{\prime}$ implies $Y \xi=0$ on $[a, b)$, in particular $\Xi \xi=0$. Hence (iii $)^{\prime \prime}$ holds.

Now we suppose $\left(i i^{\prime \prime}\right)$ to hold.

Let $c \in(a, b)$ arbitrary. We have to show that $A, B \in L^{1}(a, c)$.

Therefor let $Y$ a regular solution of (2.1) for $\lambda=0$. We know $|\operatorname{det} Y|>\varepsilon$ on $[a, c]$ for some $\varepsilon>0$, because $Y$ is continuous and nowhere zero. Thus $Y^{-1}$ is continuous. Since $Y$ is in $A C_{\text {loc }}[a, b)$, we know that $-J B Y=Y^{\prime}$ lies in $L^{1}(a, c)$, and thus also $B=J(-J B Y) Y^{-1}$.

If we put $\lambda=1$, with the same argument we obtain $-J A-J B Y \in L^{1}(a, c)$ and therefore $A \in L^{1}(a, c)$.

Since $B$ need not be self-adjoint, we introduce some kind of generalized rotation matrix for the M-function theory. This matrix corresponds to the rotation parameter $\eta$ in [6], where the non-self-adjoint scalar case is considered.

In [5] the regular matrix $\mathcal{U}_{2 n}$ was introduced for this purpose, with the property that $\mathcal{U}_{2 n} J$ is self-adjoint with as many positive eigenvalues as negative ones. Here the matrix $V$ below takes the place of $\mathcal{U}_{2 n} J$. 
Let $V \in \mathbb{C}^{n, n}$ be self-adjoint and regular. We call $V$ admissible if there exists some $\lambda, k \in \mathbb{C}$ such that the following two conditions hold:

$$
\begin{gathered}
C_{k, V}:=-\operatorname{Re}(V J(k A+B)) \succcurlyeq 0, \\
-\operatorname{Re}(\lambda V J A) \succcurlyeq A .
\end{gathered}
$$

Here, for matrix-valued functions $F, G$, the pre-order relation $F \succcurlyeq G$ means that there exists some $\delta>0$ such that $F \geq \delta G$ almost everywhere on $[a, b)$. Thus $F \succcurlyeq 0$ means $F(x) \geq 0$ for a.e. $x \in[a, b)$.

Assumption 1. There exists an admissible $V \in \mathbb{C}^{n, n}$.

This is the only assumption we need to construct an M-function, at least on the set $\operatorname{Adm}_{V}+\Lambda_{V}$, with

$$
\begin{gathered}
\operatorname{Adm}_{V}:=\left\{k \in \mathbb{C}: C_{k, V} \succcurlyeq 0\right\}, \\
\Lambda_{V}:=\{\lambda \in \mathbb{C}:-\operatorname{Re}(\lambda V J A) \succcurlyeq A\} .
\end{gathered}
$$

Admissibility of $V$ is equivalent to $\operatorname{Adm}_{V}+\Lambda_{V}$ not being empty. Sometimes we also use the short notation $W_{V}:=\operatorname{Adm}_{V}+\Lambda_{V}$.

Following the lines of [5], the condition in the definition of $\Lambda_{V}$ would read $-\boldsymbol{R e}(\lambda V J A) \succcurlyeq$ $V J A J^{*} V$. But as we will see in Theorem 3.1.3, this will amount to the same condition.

For any admissible $V$, let $n^{+}=n^{+}(V)$ and $n^{-}=n^{-}(V)$ be the numbers of positive, respectively negative eigenvalues of $V$ (counted by multiplicity). Note that $n^{+}+n^{-}=n$, since $V$ is regular.

For a regular matrix $E \in \mathbb{C}^{n, n}$, let $Y_{\lambda, E}$ be the fundamental matrix of (2.1) which satisfies $Y_{\lambda, E}(a)=E$. Furthermore, let

$$
\left(\Theta_{\lambda, n^{-}, E} \mid \Phi_{\lambda, n^{+}, E}\right):=Y_{\lambda, E}
$$

with $\Theta_{\lambda, n^{-}, E}:[a, b) \rightarrow \mathbb{C}^{n, n^{-}}$and $\Phi_{\lambda, n^{+}, E}:[a, b) \rightarrow \mathbb{C}^{n, n^{+}}$. Moreover let

$$
P_{\lambda, V, E}:=\frac{1}{2} \Phi_{\lambda, n^{+}, E}^{*} V \Phi_{\lambda, n^{+}, E} .
$$

It should be mentioned that $n^{+}=0$ or $n^{-}=0$ is allowed (with straightforward calculus). If we recall $\mathbb{C}^{0}=\{0\}$, then for arbitrary $n_{1}, n_{2} \in \mathbb{N}_{0}$, we can associate $\mathbb{C}^{n_{1}, n_{2}}$ with the set of all 
linear mappings $\mathbb{C}^{n_{2}} \rightarrow \mathbb{C}^{n_{1}}$. For a detailed definition of $\mathbb{C}^{n_{1}, n_{2}}$, see (even in more generality) [4, Chapter II, §10].

For $m \in \mathbb{N}$ we use the notation $0_{0,0}, 0_{0, m}, 0_{m, 0}$ for the only element in $\mathbb{C}^{0,0}, \mathbb{C}^{0, m}$ or $\mathbb{C}^{m, 0}$, respectively. Note that $0_{0,0}$ is regular.

We call a pair $(V, E)$ suitable if $V$ is admissible,

$$
P_{\lambda, V, E}(a)=P_{V, E}(a) \geq 0
$$

and for every $\zeta \in \mathbb{C}^{n^{+}}, \lambda \in \operatorname{Adm}_{V}+\Lambda_{V}$,

$$
\left(P_{V, E}(a) \zeta=0 \text { and }\left(C_{\lambda, V} \Phi_{\lambda, n^{+}, E}\right)(x) \zeta=0 \text { for a.e. } \mathrm{x} \in[a, b)\right) \Rightarrow \zeta=0 .
$$

Note that $P_{\lambda, V, E}(a)$ does not depend on $\lambda$.

Remark 2.1.2. Both assumptions (2.9) and (2.10) obviously hold if $P_{V, E}(a)$ is positive definite.

Lemma 2.1.3. For every admissible $V$, there exists a regular $E \in \mathbb{C}^{n, n}$ such that $(V, E)$ is suitable.

Proof. Let $\left\{\xi_{1}, \ldots, \xi_{n^{-}}, \psi_{1}, \ldots, \psi_{n^{+}}\right\}$be an orthonormal basis of eigenvectors of $V$, where $\psi_{1}, \ldots, \psi_{n^{+}}$correspond to the positive eigenvalues. If we set

$$
E:=\left(\xi_{1}|\cdots| \xi_{n^{-}}\left|\psi_{1}\right| \cdots \mid \psi_{n^{+}}\right)
$$

then $\Phi_{\lambda, n^{+}, E}(a)=\left(\psi_{1}|\cdots| \psi_{n^{+}}\right)$, and so for any $\zeta \in \mathbb{C}^{n^{+}} \backslash\{0\}$ we have

$$
\Phi_{\lambda, n^{+}, E}(a) \zeta \in \operatorname{span}\left(\psi_{1}, \ldots, \psi_{n^{+}}\right) \backslash\{0\}
$$

which implies

$$
\zeta^{*} P_{\lambda, V, E}(a) \zeta=\left(\Phi_{\lambda, n^{+}, E}(a) \zeta\right)^{*} V \Phi_{\lambda, n^{+}, E}(a) \zeta>0 .
$$

This means that $P_{V, E}(a)$ is positive definite and hence $(V, E)$ is suitable.

Now we fix a suitable pair $(V, E)$. All dependence on $V$ and on $E$ shall refer to this pair from now on unless otherwise stated. So we can omit the indices $V, E, n^{-}$and $n^{+}$.

Remark 2.1.4. Let $z \in \mathbb{C} \backslash\{0\}$. As one can easily verify, $(V, z E)$ is suitable. The whole theory below is not essentially changed by such a scalar factor for $E$, in the sense that we obtain the same $M$-function, the same differential operator and the same estimates. 


\subsection{Generalized Weyl-circles}

We define the Weyl-Sims sets for (2.1) by

$$
D_{\lambda}(x):=\left\{l \in \mathbb{C}^{n^{+}, n^{-}}:\left[\left(\Theta_{\lambda}+\Phi_{\lambda} l\right)^{*} V\left(\Theta_{\lambda}+\Phi_{\lambda} l\right)\right](x) \leq 0\right\} .
$$

These sets are obviously closed and it will turn out that they are nonempty, inclusiondecreasing in $x$ and eventually compact. For $\lambda \in \operatorname{Adm}_{V}+\Lambda_{V}$ and $M \in \bigcup_{c \in[a, b)} \bigcap_{x \in(c, b)} D_{\lambda}(x)$ we will see that $\Theta_{\lambda}+\Phi_{\lambda} M$ lies in $L_{A}^{2}$.

For the following we need the equation*1

$$
\begin{aligned}
\left.Y_{\lambda}^{*} V Y_{\lambda}\right|_{a} ^{c} & =\int_{a}^{c}\left(Y_{\lambda}^{*} V Y_{\lambda}\right)^{\prime} d x \\
& =\int_{a}^{c} Y_{\lambda}^{*} V Y_{\lambda}^{\prime}+\left(Y_{\lambda}^{*} V^{*} Y_{\lambda}^{\prime}\right)^{*} d x \\
& =2 \int_{a}^{c} \operatorname{Re}\left(Y_{\lambda}^{*} V Y_{\lambda}^{\prime}\right) d x \\
& =2 \int_{a}^{c} \operatorname{Re}\left(Y_{\lambda}^{*} V(-J) J Y_{\lambda}^{\prime}\right) d x \\
& =2 \int_{a}^{c} \operatorname{Re}\left(Y_{\lambda}^{*} V(-J)(\lambda A+B) Y_{\lambda}\right) d x \\
& =2 \int_{a}^{c} Y_{\lambda}^{*} \operatorname{Re}(-V J(\lambda A+B)) Y_{\lambda} d x \\
& =2 \int_{a}^{c} Y_{\lambda}^{*} C_{\lambda} Y_{\lambda} d x,
\end{aligned}
$$

and furthermore

Corollary 2.2.1. For $\lambda \in A d m_{V}+\Lambda_{V}$, we have

$$
C_{\lambda} \succcurlyeq A \succcurlyeq 0
$$

which is obvious by the definition of $C_{\lambda}$ and equations (2.5), (2.6). This yields

$$
\operatorname{Adm}_{V} \supseteq \operatorname{Adm}_{V}+\Lambda_{V}
$$

If we define

$$
\mathcal{A}_{\lambda}(l):=-\frac{1}{2}\left[\left(\Theta_{\lambda}+\Phi_{\lambda} l\right)^{*} V\left(\Theta_{\lambda}+\Phi_{\lambda} l\right)\right](a),
$$


for $l \in \mathbb{C}^{n^{+}, n^{-}}$, we obtain an alternative representation of $D_{\lambda}(x)$ in

\section{Lemma 2.2.2. ${ }^{{ }_{1}}$}

$$
D_{\lambda}(c)=\left\{l \in \mathbb{C}^{n^{+}, n^{-}}: \int_{a}^{c}\left(\Theta_{\lambda}+\Phi_{\lambda} l\right)^{*} C_{\lambda}\left(\Theta_{\lambda}+\Phi_{\lambda} l\right) d x \leq \mathcal{A}_{\lambda}(l)\right\} .
$$

Proof. Let $l \in \mathbb{C}^{n^{+}, n^{-}}$. Equation (2.12) yields

$$
\begin{aligned}
\left.\frac{1}{2}\left[\left(\Theta_{\lambda}+\Phi_{\lambda} l\right)^{*} V\left(\Theta_{\lambda}+\Phi_{\lambda} l\right)\right]\right|_{a} ^{c} & =\left.\frac{1}{2}\left(I_{n^{-}} \mid l^{*}\right)\left[Y_{\lambda}^{*} V Y_{\lambda}\right]\right|_{a} ^{c}\left(\begin{array}{c}
I_{n^{-}} \\
l
\end{array}\right) \\
& =\left(I_{n^{-}} \mid l^{*}\right) \int_{a}^{c} Y_{\lambda}^{*} C_{\lambda} Y_{\lambda} d x\left(\begin{array}{c}
I_{n^{-}} \\
l
\end{array}\right) \\
& =\int_{a}^{c}\left(\Theta_{\lambda}+\Phi_{\lambda} l\right)^{*} C_{\lambda}\left(\Theta_{\lambda}+\Phi_{\lambda} l\right) d x
\end{aligned}
$$

Subtracting $\mathcal{A}_{\lambda}(l)$ shows that

$$
\int_{a}^{c}\left(\Theta_{\lambda}+\Phi_{\lambda} l\right)^{*} C_{\lambda}\left(\Theta_{\lambda}+\Phi_{\lambda} l\right) d x-\mathcal{A}_{\lambda}(l) \leq 0
$$

if and only if

$$
\left[\left(\Theta_{\lambda}+\Phi_{\lambda} l\right)^{*} V\left(\Theta_{\lambda}+\Phi_{\lambda} l\right)\right](c) \leq 0
$$

By definition, this is equivalent to $l \in D_{\lambda}(c)$.

Using this Lemma, we obtain

Corollary 2.2.3. ${ }^{*_{1}}$ For $\lambda \in A d m_{V}+\Lambda_{V}$ and $c, d \in[a, b)$ holds

$$
c<d \Longrightarrow D_{\lambda}(c) \supseteq D_{\lambda}(d) \text {. }
$$

Proof. Let $l \in D_{\lambda}(d)$. Using (2.16) and $C_{\lambda} \geq 0$ almost everywhere on $[a, b)$, we can estimate

$$
\mathcal{A}_{\lambda}(l) \geq \int_{a}^{d}\left(\Theta_{\lambda}+\Phi_{\lambda} l\right)^{*} C_{\lambda}\left(\Theta_{\lambda}+\Phi_{\lambda} l\right) d x \geq \int_{a}^{c}\left(\Theta_{\lambda}+\Phi_{\lambda} l\right)^{*} C_{\lambda}\left(\Theta_{\lambda}+\Phi_{\lambda} l\right) d x
$$

Again (2.16) shows $l \in D_{\lambda}(c)$.

As in [5] we define correspondingly

$$
\left(\begin{array}{cc}
S_{\lambda} & T_{\lambda} \\
T_{\lambda}^{*} & P_{\lambda}
\end{array}\right):=\frac{1}{2} Y_{\lambda}^{*} V Y_{\lambda}=\frac{1}{2}\left(\begin{array}{cc}
\Theta_{\lambda}^{*} V \Theta_{\lambda} & \Theta_{\lambda}^{*} V \Phi_{\lambda} \\
\Phi_{\lambda}^{*} V \Theta_{\lambda} & \Phi_{\lambda}^{*} V \Phi_{\lambda}
\end{array}\right)
$$


with $P_{\lambda}(x) \in \mathbb{C}^{n^{+}, n^{+}}, S_{\lambda}(x) \in \mathbb{C}^{n^{-}, n^{-}}$and $T_{\lambda}(x) \in \mathbb{C}^{n^{-}, n^{+}}$for $x \in[a, b)$.

Note that $P_{\lambda}$ and $S_{\lambda}$ are self-adjoint, and (2.18) is consistent with the former definition of $P_{\lambda}$. So equation $(2.12)$ reads

$$
\left.\left(\begin{array}{cc}
S_{\lambda} & T_{\lambda} \\
T_{\lambda}^{*} & P_{\lambda}
\end{array}\right)\right|_{a} ^{c}=\int_{a}^{c} Y_{\lambda}^{*} C_{\lambda} Y_{\lambda} d x=\int_{a}^{c}\left(\begin{array}{cc}
\Theta_{\lambda}^{*} C_{\lambda} \Theta_{\lambda} & \Theta_{\lambda}^{*} C_{\lambda} \Phi_{\lambda} \\
\Phi_{\lambda}^{*} C_{\lambda} \Theta_{\lambda} & \Phi_{\lambda}^{*} C_{\lambda} \Phi_{\lambda}
\end{array}\right) d x
$$

Lemma 2.2.4. ${ }^{*}$ For $\lambda \in A d m_{V}+\Lambda_{V}$ :

(i) $P_{\lambda}$ is non-decreasing,

(ii) $P_{\lambda}(c) \geq 0$ for $c \in[a, b)$,

(iii) There exists $c_{0}(\lambda)$ such that $P_{\lambda}(c)$ is regular for $c \geq c_{0}(\lambda)$.

Proof. We can assume $n^{+}>0$ w.l.o.g. because otherwise $P_{\lambda}=0_{0,0}$ and thus all statements are true.

Equation (2.19) implies

$$
\left.P_{\lambda}\right|_{a} ^{c}=\int_{a}^{c} \Phi_{\lambda}^{*} C_{\lambda} \Phi_{\lambda} d x
$$

which shows that $P_{\lambda}$ is nondecreasing since $C_{\lambda}(x) \geq 0$ for a.e. $x \in[a, b)$ by (2.13). Due to $P_{\lambda}(a) \geq 0$, we conclude that $P_{\lambda}(c) \geq 0$ for all $c \in[a, b)$.

To prove that $P_{\lambda}>0$ eventually, we assume, on the contrary, that there exist sequences $\left(x_{m}\right)$ with $x_{m} \rightarrow b$ and $\left(\zeta_{m}\right)$ such that $P_{\lambda}\left(x_{m}\right) \zeta_{m}=0$ and $\zeta_{m}^{*} \zeta_{m}=1$ for $m \in \mathbb{N}$. W.l.o.g., $\left(\zeta_{m}\right)$ converges (otherwise we could extract a convergent subsequence).

Let $\zeta \in \mathbb{C}^{n^{+}}$the limit, which is nonzero. Since $P_{\lambda}$ is positive semi-definite and nondecreasing, $\zeta_{m}$ lies in the null-space of $P_{\lambda}(x)$ for every $m \in \mathbb{N}$ and $x \in\left[a, x_{m}\right]$ and so $\zeta$ lies in the nullspace of $P_{\lambda}(x)$ for every $x \in[a, b)$.

Thus, using $C_{\lambda}(x) \geq 0$ and (2.20) we have

$$
P_{\lambda}(a) \zeta=0, \quad \zeta^{*}\left(\Phi_{\lambda}^{*} C_{\lambda} \Phi_{\lambda}\right)(x) \zeta=0 \text { for a.e. } \mathrm{x} \in[a, b) .
$$

Taking into account that $C_{\lambda}$ is self-adjoint, this creates a contradiction to (2.10).

Lemma 2.2.5. ${ }^{* 1}$ For $\lambda \in A d m_{V}+\Lambda_{V}$ and $x \in[a, b)$ the set $D_{\lambda}(x)$ is nonempty.

Proof. We can assume w.l.o.g $n^{-}>0$. Otherwise $0_{n, 0} \in D_{\lambda}(x)$, since the inequality in the brackets of $(2.11)$ reads $0_{0,0} \leq 0_{0,0}$. 
Definition (2.18) shows that $n^{-}$eigenvalues of $\left(\begin{array}{ll}S_{\lambda} & T_{\lambda} \\ T_{\lambda}^{*} & P_{\lambda}\end{array}\right)(x)$ are negative since this holds for $V$ and $Y_{\lambda}$ is regular.

Let the columns of $\left(\begin{array}{l}\Xi_{1} \\ \Xi_{2}\end{array}\right) \in \mathbb{C}^{n, n^{-}}$be corresponding orthonormal eigenvectors with $\Xi_{1} \in \mathbb{C}^{n^{-}, n^{-}}$ and $\Xi_{2} \in \mathbb{C}^{n^{+}, n^{-}}$. Then,

$$
\left(\Xi_{1}^{*} \mid \Xi_{2}^{*}\right)\left(\begin{array}{cc}
S_{\lambda} & T_{\lambda} \\
T_{\lambda}^{*} & P_{\lambda}
\end{array}\right)(x)\left(\begin{array}{c}
\Xi_{1} \\
\Xi_{2}
\end{array}\right)<0
$$

If $\Xi_{1}$ was not regular, some non-trivial $\zeta \in \mathbb{C}^{n^{-}}$would exist with $\Xi_{1} \zeta=0$. Since $\zeta$ is not zero, (2.21) and Lemma 2.2.4 (ii) would yield

$$
\begin{aligned}
0 & >\zeta^{*}\left(\Xi_{1}^{*} \mid \Xi_{2}^{*}\right)\left(\begin{array}{ll}
S_{\lambda} & T_{\lambda} \\
T_{\lambda}^{*} & P_{\lambda}
\end{array}\right)(x)\left(\begin{array}{c}
\Xi_{1} \\
\Xi_{2}
\end{array}\right) \zeta \\
& =\left(0 \mid \zeta^{*} \Xi_{2}^{*}\right)\left(\begin{array}{ll}
S_{\lambda} & T_{\lambda} \\
T_{\lambda}^{*} & P_{\lambda}
\end{array}\right)(x)\left(\begin{array}{c}
0 \\
\Xi_{2} \zeta
\end{array}\right) \\
& =\zeta^{*} \Xi_{2}^{*} P_{\lambda}(x) \Xi_{2} \zeta \geq 0 .
\end{aligned}
$$

This contradiction implies that $\Xi_{1}$ is regular.

On multiplying (2.21) by $\left(\Xi_{1}^{-1}\right)^{*}$ and $\Xi_{1}^{-1}$ from the left and the right, respectively, we obtain

$$
\left(I_{n^{-}} \mid l^{*}\right)\left(\begin{array}{cc}
S_{\lambda} & T_{\lambda} \\
T_{\lambda}^{*} & P_{\lambda}
\end{array}\right)(x)\left(\begin{array}{c}
I_{n^{-}} \\
l
\end{array}\right) \leq 0
$$

with $l:=\left(\Xi_{2} \Xi_{1}^{-1}\right)(x)$. Thus, $l \in D_{\lambda}(x)$ by $(2.18)$ and $(2.11)$.

Recall equation (2.11):

$$
D_{\lambda}(x)=\left\{l \in \mathbb{C}^{n^{+}, n^{-}}:\left[\left(\Theta_{\lambda}+\Phi_{\lambda} l\right)^{*} V\left(\Theta_{\lambda}+\Phi_{\lambda} l\right)\right](x) \leq 0\right\} .
$$

Using (2.18), we obtain

$$
\frac{1}{2}\left(\Theta_{\lambda}+\Phi_{\lambda} l\right)^{*} V\left(\Theta_{\lambda}+\Phi_{\lambda} l\right)=\left(l^{*} P_{\lambda} l+T_{\lambda} l+l^{*} T_{\lambda}^{*}+S_{\lambda}\right) .
$$

For $\lambda \in \operatorname{Adm}_{V}+\Lambda_{V}$, on $\left[c_{0}(\lambda), b\right)$ we use the notation

$$
\begin{gathered}
\mathcal{C}_{\lambda}:=-P_{\lambda}^{-1} T_{\lambda}^{*}, \\
\mathcal{R}_{\lambda}:=T_{\lambda} P_{\lambda}^{-1} T_{\lambda}^{*}-S_{\lambda},
\end{gathered}
$$


in which case

$$
\left(\Theta_{\lambda}+\Phi_{\lambda} l\right)^{*} V\left(\Theta_{\lambda}+\Phi_{\lambda} l\right)=2\left(\left(l-\mathcal{C}_{\lambda}\right)^{*} P_{\lambda}\left(l-\mathcal{C}_{\lambda}\right)-\mathcal{R}_{\lambda}\right)
$$

Thus, for $x \in\left[c_{0}(\lambda), b\right)$,

$$
\begin{aligned}
D_{\lambda}(x) & =\mathcal{C}_{\lambda}(x)+\left\{l \in \mathbb{C}^{n^{+}, n^{-}}: l^{*} P_{\lambda}(x) l \leq \mathcal{R}_{\lambda}(x)\right\} \\
& =\mathcal{C}_{\lambda}(x)+\left\{P_{\lambda}^{-1 / 2}(x) N \mathcal{R}_{\lambda}^{1 / 2}(x): N \in \mathbb{C}^{n^{+}, n^{-}} \text {with } N^{*} N \leq I_{n^{-}}\right\} .
\end{aligned}
$$

Note that the last expression makes sense because of the following

Lemma 2.2.6. ${ }^{* 1}$ Let $\lambda \in A d m_{V}+\Lambda_{V}$. $\mathcal{R}_{\lambda}$ is non-increasing on $\left[c_{0}(\lambda), b\right)$. If $n^{-}>0$ then $\mathcal{R}_{\lambda}(x)>0$ for $x \in\left[c_{0}(\lambda), b\right)$.

Proof. On using (2.25), (2.19) and (2.24), we obtain

$$
\begin{aligned}
\mathcal{R}_{\lambda}^{\prime} & =T_{\lambda}^{\prime} P_{\lambda}^{-1} T_{\lambda}^{*}+T_{\lambda} P_{\lambda}^{-1}\left(T_{\lambda}^{*}\right)^{\prime}-T_{\lambda} P_{\lambda}^{-1} P_{\lambda}^{\prime} P_{\lambda}^{-1} T_{\lambda}^{*}-S_{\lambda}^{\prime} \\
& =\Theta_{\lambda}^{*} C_{\lambda} \Phi_{\lambda} P_{\lambda}^{-1} T_{\lambda}^{*}+T_{\lambda} P_{\lambda}^{-1} \Phi_{\lambda}^{*} C_{\lambda} \Theta_{\lambda}-T_{\lambda} P_{\lambda}^{-1} \Phi_{\lambda}^{*} C_{\lambda} \Phi_{\lambda} P_{\lambda}^{-1} T_{\lambda}^{*}-\Theta_{\lambda}^{*} C_{\lambda} \Theta_{\lambda} \\
& =-\left(\Theta_{\lambda}+\Phi_{\lambda} \mathcal{C}_{\lambda}\right)^{*} C_{\lambda}\left(\Theta_{\lambda}+\Phi_{\lambda} \mathcal{C}_{\lambda}\right) .
\end{aligned}
$$

It follows from (2.13) that $\mathcal{R}_{\lambda}^{\prime}(x) \leq 0$ for a.e. $x \in[a, b)$, and consequently $\mathcal{R}_{\lambda}$ is nonincreasing.

Moreover, $\mathcal{R}_{\lambda} \geq 0$ by (2.26) since $D_{\lambda}(x)$ is nonempty and $P_{\lambda}(x) \geq 0$.

It remains to show that $\mathcal{R}_{\lambda}$ is regular. $\left(\begin{array}{ll}S_{\lambda} & T_{\lambda} \\ T_{\lambda}^{*} & P_{\lambda}\end{array}\right)$ is pointwise regular by definition (2.18) as a product of regular matrices. Let

$$
\left(\begin{array}{l}
N^{-} \\
N^{+}
\end{array}\right) \in \mathbb{C}^{n, n^{-}}
$$

be such that

$$
\left(\begin{array}{ll}
S_{\lambda} & T_{\lambda} \\
T_{\lambda}^{*} & P_{\lambda}
\end{array}\right)\left(\begin{array}{l}
N^{-} \\
N^{+}
\end{array}\right)=\left(\begin{array}{l}
I_{n^{-}} \\
0_{n^{+}}
\end{array}\right) .
$$

This yields

$$
N^{+}=-P_{\lambda}^{-1} T_{\lambda}^{*} N^{-}
$$

and with (2.25),

$$
\mathcal{R}_{\lambda} N^{-}=-I_{n^{-}} .
$$

So $\mathcal{R}_{\lambda}$ is invertible, whence $\mathcal{R}_{\lambda}>0$. 
Theorem 2.2.7. ${ }^{*_{2}}$ Let $\lambda \in A d m_{V}+\Lambda_{V}$. For $c, d \in\left[c_{0}(\lambda), b\right)$ holds:

(i) $D_{\lambda}(c)$ is compact and convex,

(ii) $c<d \Longrightarrow D_{\lambda}(c)-\mathcal{C}_{\lambda}(c) \supseteq D_{\lambda}(d)-\mathcal{C}_{\lambda}(d)$,

(iii) $\mathcal{C}_{\lambda}(b):=\lim _{x \rightarrow b} \mathcal{C}_{\lambda}(x)$ exists,

(iv) $\bigcap_{x \in\left[c_{0}, b\right)}\left(D_{\lambda}(x)-\mathcal{C}_{\lambda}(x)\right)=D_{\lambda}(b)-\mathcal{C}_{\lambda}(b)$ with $D_{\lambda}(b):=\bigcap_{x \in\left[c_{0}, b\right)} D_{\lambda}(x)$,

(v) $\mathcal{C}_{\lambda}(b) \in D_{\lambda}(b)$

Proof. By Lemma 2.2.5, the cases $n^{-}=0$ or $n^{+}=0$ are trivial, so we can exclude them for the proof.

(i) Equation (2.27) yields closedness and boundedness of $D_{\lambda}(c)$. So it is compact as a subset of a finite dimensional space.

Using the triangle inequality in $\mathbb{C}^{n^{+}}$, we obtain convexity of $\left\{N \in \mathbb{C}^{n^{+}, n^{-}}: N^{*} N \leq I_{n^{-}}\right\}$and, with (2.27) again, convexity of $D_{\lambda}(c)$.

(ii) Let $l \in D_{\lambda}(c)-\mathcal{C}_{\lambda}(c)$; then (2.26) gives $l^{*} P_{\lambda}(c) l \leq \mathcal{R}_{\lambda}(c)$. Since $P_{\lambda}$ is increasing and $\mathcal{R}_{\lambda}$ is decreasing by Lemmata 2.2.4 and 2.2.6 we have $l^{*} P_{\lambda}(d) l \leq l^{*} P_{\lambda}(c) l \leq \mathcal{R}_{\lambda}(c) \leq \mathcal{R}_{\lambda}(d)$. Again equation (2.26) implies $l \in D_{\lambda}(d)-\mathcal{C}_{\lambda}(d)$.

(iii) We associate $\mathbb{C}^{n^{+}, n^{-}}$with $\mathbb{C}^{n^{+} n^{-}}$(for the scalar product).

If $\left(\mathcal{C}_{\lambda}(x)\right)$ would not converge for $x \rightarrow b$, there would be an ascending sequence $\left(x_{m}\right)$ in $\left[c_{0}, b\right)$ with $\mathcal{C}_{\lambda}\left(x_{2 m}\right) \rightarrow \mathcal{C}_{1}$ and $\mathcal{C}_{\lambda}\left(x_{2 m-1}\right) \rightarrow \mathcal{C}_{2}$ for two points $\mathcal{C}_{1} \neq \mathcal{C}_{2} \in \mathbb{C}^{n^{+}, n^{-}}$.

We define the continuous linear mapping $\left.\varphi: \mathbb{C}^{n^{+}, n^{-}} \rightarrow \mathbb{R}, x \mapsto \operatorname{Re}\left(<x, \overline{\mathcal{C}_{1}-\mathcal{C}_{2}}\right\rangle\right)$.

Since $\varphi\left(\mathcal{C}_{1}\right)-\varphi\left(\mathcal{C}_{2}\right)=\varphi\left(\mathcal{C}_{1}-\mathcal{C}_{2}\right)>0$ we can assume w.l.o.g. that $\left|\varphi\left(\mathcal{C}_{\lambda}\left(x_{m}\right)\right)-\varphi\left(\mathcal{C}_{\lambda}\left(x_{m+1}\right)\right)\right| \geq$ $\varepsilon>0$ for all $m \in \mathbb{N}$.

$\varphi\left(D_{\lambda}\left(x_{1}\right)\right)$ is a bounded interval. From $(i i)$ we know

$$
D_{\lambda}\left(x_{m+1}\right) \subseteq D_{\lambda}\left(x_{m}\right)-\mathcal{C}_{\lambda}\left(x_{m}\right)+\mathcal{C}_{\lambda}\left(x_{m+1}\right)
$$

With Corollary 2.2.3 we can conclude

$$
\varphi\left(D_{\lambda}\left(x_{m+1}\right)\right) \subseteq \varphi\left(D_{\lambda}\left(x_{m}\right)\right) \cap\left(\varphi\left(D_{\lambda}\left(x_{m}\right)\right)-\left(\varphi\left(\mathcal{C}_{\lambda}\left(x_{m}\right)\right)-\varphi\left(\mathcal{C}_{\lambda}\left(x_{m+1}\right)\right)\right)\right),
$$

where the right-hand side is an interval of length at least $\varepsilon$ less than the length of $\varphi\left(D_{\lambda}\left(x_{m}\right)\right)$. So there is an $m \in \mathbb{N}$ such that $\varphi\left(D_{\lambda}\left(x_{m}\right)\right)$ is empty which means $D_{\lambda}\left(x_{m}\right)$ is empty. This contradicts Lemma 2.2.5.

Thus $\left(\mathcal{C}_{\lambda}(x)\right)$ converges as $x \rightarrow b$. 
(iv) Let $\mu \in \bigcap_{x \in\left[c_{0}, b\right)}\left(D_{\lambda}(x)-\mathcal{C}_{\lambda}(x)\right)$. Then for $x \in\left[c_{0}, b\right)$,

$$
\mu=l_{x}-C_{\lambda}(x)
$$

for some $l_{x} \in D_{\lambda}(x)$. Since $\mathcal{C}_{\lambda}(x) \rightarrow \mathcal{C}_{\lambda}(b)$ as $x \rightarrow b$ we obtain $l_{x} \rightarrow l_{b}:=\mu+\mathcal{C}_{\lambda}(b)$ and $l_{b} \in \bigcap_{x \in\left[c_{0}, b\right)} D_{\lambda}(x)$ in view of Corollary 2.2.3 and the closedness of $D_{\lambda}(x)$. Thus

$$
\mu=l_{b}-\mathcal{C}_{\lambda}(b) \in D_{\lambda}(b)-\mathcal{C}_{\lambda}(b)
$$

Conversely let $\mu=l_{b}-\mathcal{C}_{\lambda}(b)$ with $l_{b} \in \bigcap_{x \in\left[c_{0}, b\right)} D_{\lambda}(x)$.

Then $\nu(x):=l_{b}-\mathcal{C}_{\lambda}(x) \rightarrow \mu$ as $x \rightarrow b$, and $\nu(x) \in D_{\lambda}(x)-\mathcal{C}_{\lambda}(x)$ for all $x \in\left[c_{0}, b\right)$. By $(i i)$ and the closedness of $D_{\lambda}(x)$, we obtain $\mu \in \bigcap_{x \in\left[c_{0}, b\right)}\left(D_{\lambda}(x)-\mathcal{C}_{\lambda}(x)\right)$.

(v) By $(2.27), \mathcal{C}_{\lambda}(x) \in D_{\lambda}(x)$ (choose $N=0$ ). So, with $(i v)$, we know that $0 \in D_{\lambda}(b)-$ $\mathcal{C}_{\lambda}(b)$, which yields the statement.

Theorem 2.2.7 and Corollary 2.2.3 show the behavior of these generalized Weyl-Sims circles. Already in [1, section 9.10] Atkinson observed the intersection of these kinds of Weyl-Sims sets. Those set replaces the circular disk.

According to the shape of $D_{\lambda}(b)$ we will later classify the problem. As we will see this classification does not depend on the actual choice of $\lambda$ in $\operatorname{Adm}_{V}+\Lambda_{V}$.

We will say that problem (2.1) is in the limit-point case, if $D_{\lambda}(b)$ consists of one point.

We will say that problem $(2.1)$ is in the limit-circle case, if $n^{+}>0$ and the span of $\{\Xi \xi: \Xi \in$ $\left.D_{\lambda}(b)-C_{\lambda}(b), \xi \in \mathbb{C}^{n^{-}}\right\}$is the whole $\mathbb{C}^{n^{+}}$.

It will turn out that there are cases in between, and even further distinctions are useful.

\subsection{Examples}

Throughout this thesis we will repeatedly consider the special case that $B(x)$ is self-adjoint for all $x \in[a, b)$. This is the setting of [12]. The theory therein does not require any matrix corresponding to $V$. To achieve the same results as in [12], we can choose the admissible matrices $V= \pm i J$, which we call the canonical choices of $V$ for the self-adjoint case (i.e. for the case that $B$ is self-adjoint). 
With this choice, we obtain $C_{0, V_{1 / 2}}=0$ and $\Lambda_{V_{1 / 2}}=\mathrm{Adm}_{V_{1 / 2}}+\Lambda_{V_{1 / 2}}$, which is respectively the lower or the upper halfplane. For the calculations see sections 6.1 and 6.2 below.

We furthermore consider the even-order scalar differential equation

$$
\tau_{e}[v]:=p_{0} v+\sum_{k=1}^{m}(-1)^{k}\left(\left(p_{k} v^{(k)}\right)^{(k)}-\left(q_{k} v^{(k)}\right)^{(k-1)}-\left(r_{k} v^{(k-1)}\right)^{(k)}\right)=\lambda w v
$$

and the odd-order scalar differential equation

$$
\begin{aligned}
\tau_{o}[v]: & =p_{0} v+(-1)^{m} i\left(s\left(s v^{(m)}\right)^{\prime}\right)^{(m)}+\sum_{k=1}^{m}(-1)^{k}\left(\left(p_{k} v^{(k)}\right)^{(k)}-\left(q_{k} v^{(k)}\right)^{(k-1)}-\left(r_{k} v^{(k-1)}\right)^{(k)}\right) \\
& =\lambda w v
\end{aligned}
$$

where all coefficient functions (except those for the highest derivate) are locally integrable over $[a, b)$. The coefficient function for the highest derivate $s$, respectively $p_{m}$ in the even case, is a.e. non-zero. Furthermore $\frac{1}{s}$, respectively $\frac{1}{p_{m}}$ in the even case, is locally integrable. $w$ is a non-negative weight function on $[a, b)$, furthermore $w>0$ on a set of positive measure.

We first examine the even case (2.31).

In the style of [3], to obtain a Hamiltonian system, we introduce the following quasiderivatives:

$$
\begin{aligned}
v^{[j]}:=v^{(j)} & \text { for } 0 \leq j \leq m-1, \\
v^{[m]}:=p_{m} v^{(m)}-r_{m} v^{(m-1)}, &
\end{aligned}
$$

and for $1 \leq j \leq m-1$

$$
\begin{aligned}
v^{[m+j]} & :=-\left(v^{[m+j-1]}\right)^{\prime}+p_{m-j} v^{(m-j)}-r_{m-j} v^{(m-j-1)}+q_{m-j+1} v^{(m-j+1)} \\
& =\sum_{k=m-j}^{m}(-1)^{k-m+j}\left(p_{k} v^{(k)}-r_{k} v^{(k-1)}\right)^{(k-m+j)}+\sum_{k=m-j+1}^{m}(-1)^{k-1-m+j}\left(q_{k} v^{(k)}\right)^{(k-1-m+j)} .
\end{aligned}
$$

This yields

$$
\tau_{e}[v]=-\left(v^{[2 m-1]}\right)^{\prime}+p_{0} v^{[0]}+q_{1} v^{[1]},
$$

except for $m=1$, since here $v^{[1]} \neq v^{(1)}$. In this case,

$$
\tau_{e}[v]=-\left(v^{[1]}\right)^{\prime}+\left(p_{0}+\frac{q_{1} r_{1}}{p_{1}}\right) v^{[0]}-\frac{q_{1}}{p_{1}} v^{[1]} .
$$

We set $y:=\left(v^{[0]}, v^{[1]}, \ldots, v^{[2 m-1]}\right)^{T}$ (here we deviate from [3], where the shape of $J$ is prescribed and therefore this setting would not match). So the equation $\tau_{e}[v]=\lambda w v$ is equivalent to $y^{\prime}=\lambda M_{A}^{e} y+M_{B}^{e} y$ for certain matrices $M_{A}^{e}, M_{B}^{e}$. 
Indeed, using the abbreviation

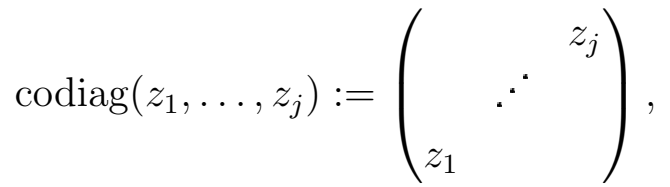

we have, for $m \geq 2, M_{A}^{e}=\operatorname{codiag}(-w, 0, \ldots, 0)$ and

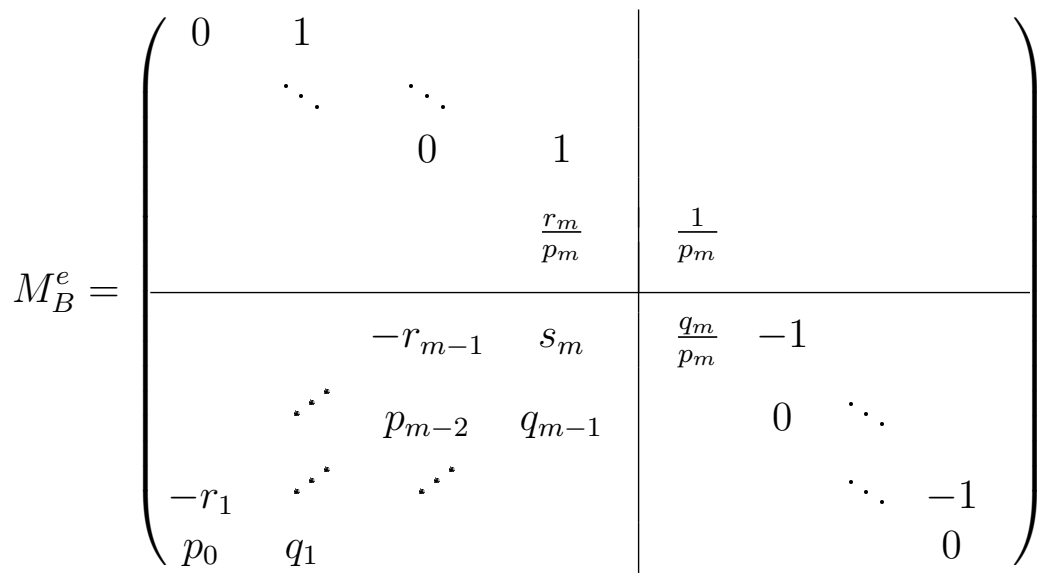

with $s_{m}:=\frac{q_{m} r_{m}}{p_{m}}+p_{m-1}$.

We set

$$
J_{1}:=\operatorname{codiag}(1, \ldots, 1,-1, \ldots,-1),
$$

obtaining the required Hamiltonian System

$$
J_{1} y^{\prime}=\left(\lambda A_{1}+B_{1}\right) y,
$$

with

$$
A_{1}:=J_{1} M_{A}^{e}=\operatorname{diag}(w, 0, \ldots, 0),
$$

and

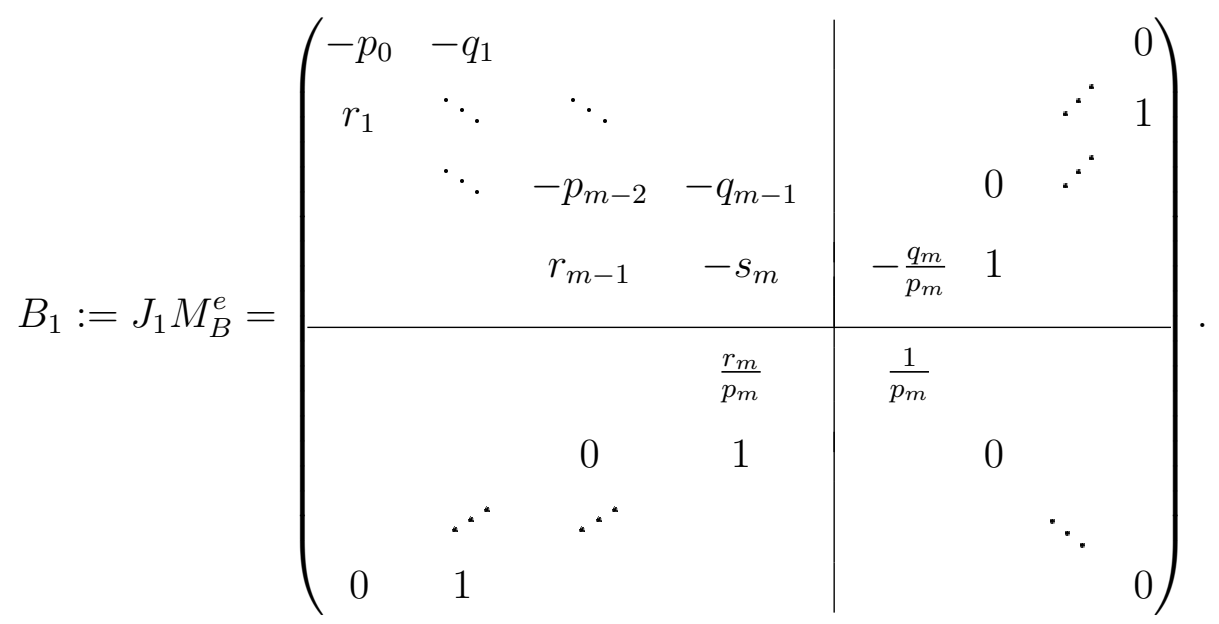


For $m=1$ the matrices $M_{A}^{e}$ and $A_{1}$ are of the same shape, with $J_{1}:=\left(\begin{array}{cc}0 & -1 \\ 1 & 0\end{array}\right)$, but

$$
M_{B}^{e}=\frac{1}{p_{1}}\left(\begin{array}{cc}
r_{1} & 1 \\
q_{1} r_{1}+p_{1} p_{0} & q_{1}
\end{array}\right) \quad \text { and } \quad B_{1}=\frac{1}{p_{1}}\left(\begin{array}{cc}
-q_{1} r_{1}-p_{1} p_{0} & -q_{1} \\
r_{1} & 1
\end{array}\right)
$$

For the odd case, we slightly deviate from [3], defining the quasi-derivatives as follows:

$$
\begin{aligned}
v^{[j]} & :=v^{(j)} \quad \text { for } 0 \leq j \leq m-1, \\
v^{[m]} & :=i s v^{(m)}, \\
v^{[m+1]} & :=i s\left(v^{[m]}\right)^{\prime}+i\left(\frac{p_{m}}{i s} v^{[m]}-r_{m} v^{[m-1]}\right)=-s\left(s v^{(m)}\right)^{\prime}+i p_{m} v^{(m)}-i r_{m} v^{(m-1)}
\end{aligned}
$$

and for $1 \leq j \leq m$

$$
\begin{aligned}
v^{[m+j+1]}:= & -\left(v^{[m+j]}\right)^{\prime}+i\left(p_{m-j} v^{(m-j)}-r_{m-j} v^{(m-j-1)}+q_{m-j+1} v^{(m-j+1)}\right) \\
= & (-1)^{j+1}\left(s\left(s v^{(m)}\right)^{\prime}\right)^{(j)}+i\left(\sum_{k=m-j}^{m}(-1)^{k-m+j}\left(p_{k} v^{(k)}-r_{k} v^{(k-1)}\right)^{(k-m+j)}\right. \\
& \left.+\sum_{k=m-j+1}^{m}(-1)^{k-1-m+j}\left(q_{k} v^{(k)}\right)^{(k-1-m+j)}\right) .
\end{aligned}
$$

This yields

$$
\tau_{o}[v]=i\left(v^{[2 m]}\right)^{\prime}+p_{0} v^{[0]}+q_{1} v^{(1)} .
$$

Finally we set $y:=\left(v^{[0]}, \ldots, v^{[2 m]}\right)^{T}$ and

$$
J_{1}:=\operatorname{codiag}(i, \ldots, i)
$$

to obtain

$$
\tau_{o}[v]=\lambda w \quad \Longleftrightarrow \quad y^{\prime}=\left(\lambda M_{o}^{1}+M_{o}^{2}\right) y \quad \Longleftrightarrow \quad J_{1} y^{\prime}=\left(\lambda A_{1}+B_{1}\right) y
$$

where $M_{A}^{o}=\operatorname{codiag}(-i w, 0, \ldots, 0)$ and $A_{1}:=J_{1} M_{A}^{o}=\operatorname{diag}(w, 0, \ldots, 0)$, furthermore 


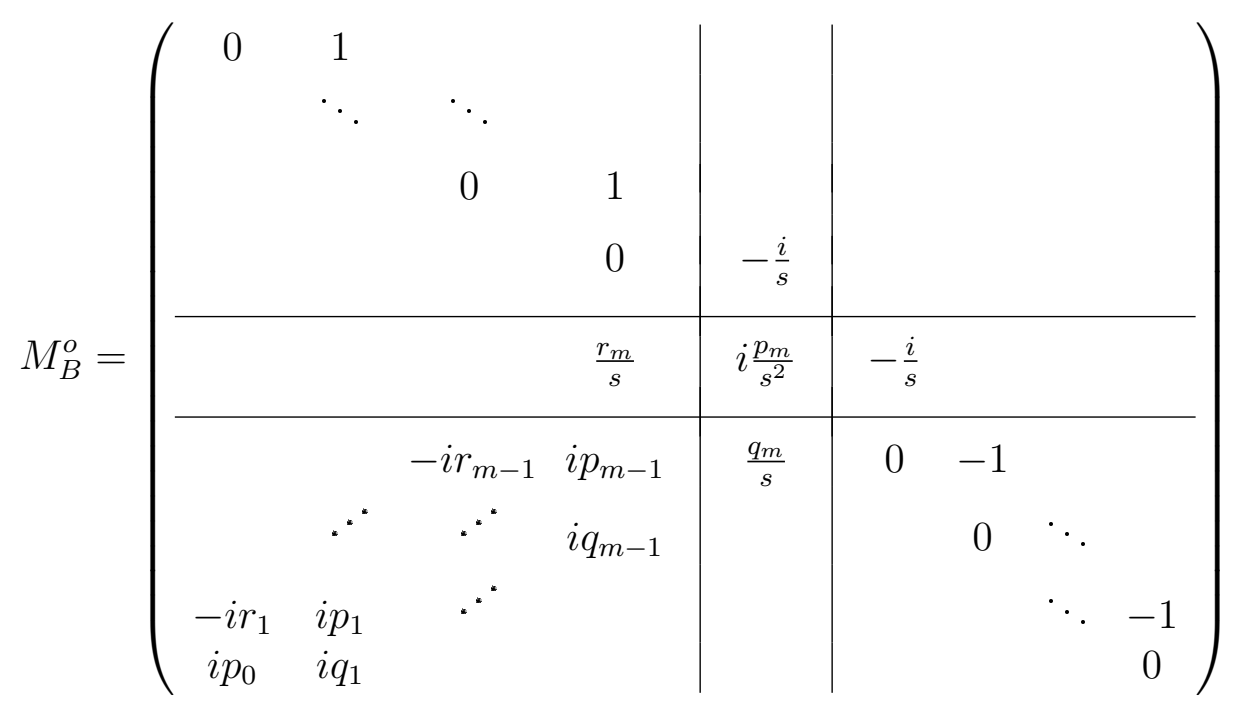

and

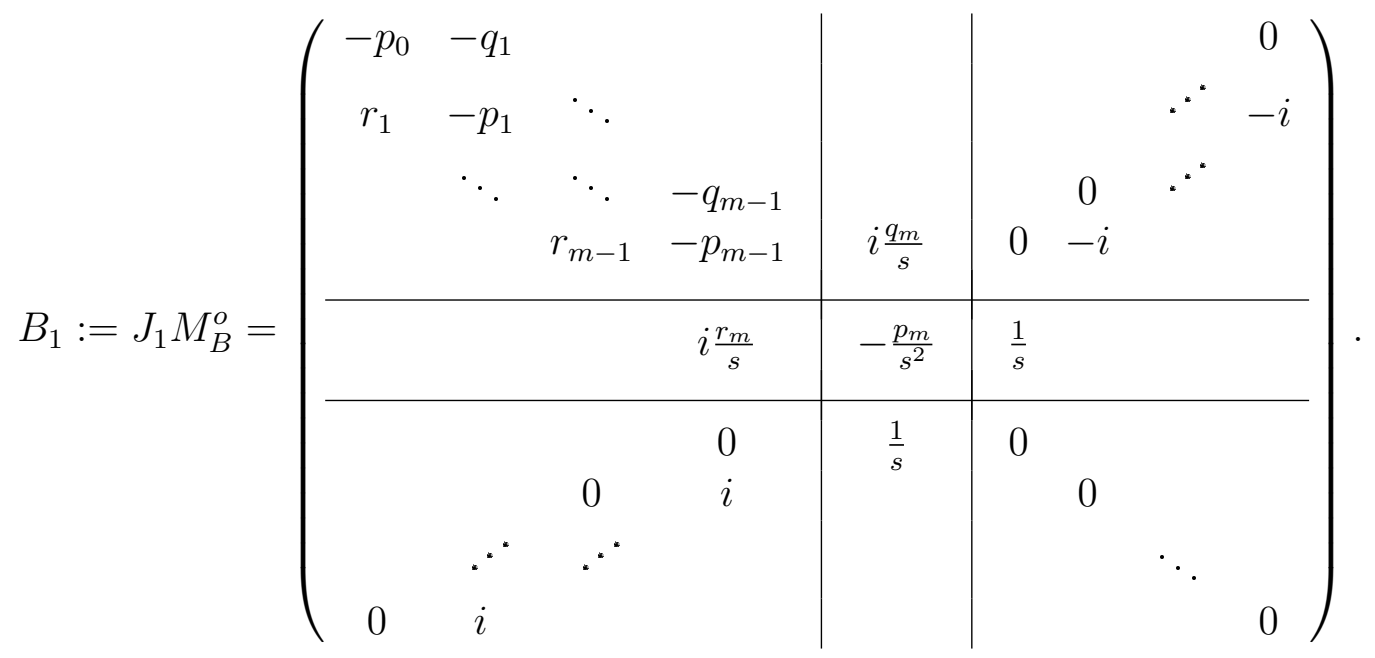

The case $m=1$ is no exception for the structure of $M_{B}^{o}$ and $B_{1}$. They consist of the $3 \times 3$ matrices in the middle of the matrices above.

In the following we like to examine the two problems in several cases with regard to the existence of suitable pairs. For each suitable pair we estimate the set $\operatorname{Adm}_{V}+\Lambda_{V}$.

In the first part we consider the case that $B$ is self-adjoint, firstly for the odd-order case, then for the even-order case. We furthermore show, within an example, that the actual choice of $J$ is less important for the theory of this thesis.

In the second part the case $m=1$ for the odd-order problem is considered, and in most generality the case $m=1$ for the even-order case in the third part. 
1. We like to consider the case that $B$ is self-adjoint. So we are in the setting of [12].

We already know that the canonical choices $V= \pm i J$ are admissible. For these choices we further know that $\operatorname{Adm}_{V}+\Lambda_{V}$ is the lower, respectively the upper halfplane.

For the choice $V=+i J$ (thus $\operatorname{Adm}_{V}+\Lambda_{V}$ is the lower halfplane), we are now searching for regular matrices $E$, such that $(V, E)$ is suitable. Such matrices $E$ exist by Lemma 2.1.3. The proof of this Lemma is constructive. Hence we can, and will use the construction therein.

1.1 In the odd case, the matrix $B_{1}$ is self-adjoint if and only if $s$ is real on $[a, b)$ and

$$
\text { for all } j \in\{1, \ldots, m\}, x \in[a, b) \text { holds } \bar{r}_{j}(x)=-q_{j}(x), p_{j}(x) \in \mathbb{R} \text {, and } p_{0}(x) \in \mathbb{R} \text {. }
$$

All this holds true in the setting of [3].

With $J_{1}$ chosen according to $(2.37)$, we obtain $V_{c}:=V_{c, 1}:=\operatorname{codiag}(-1, \ldots,-1)$, with eigenvectors $e_{j}+e_{2 m+2-j}$ (for $j \leq m$ ) and $e_{m+1}$ associated with the eigenvalue -1 , and eigenvectors $e_{j}-e_{2 m+2-j}($ for $j \leq m)$ associated with the eigenvalue +1 .

Thus we have $n^{+}=m, n^{-}=m+1$. Furthermore, with $E_{c}:=E_{c, 1}:=\operatorname{diag}(1, \ldots, 1,0,-1, \ldots,-1)+$ $\operatorname{codiag}(1, \ldots, 1)$ the pair $\left(V_{c}, E_{c}\right)$ is suitable according to the construction in the proof of Lemma 2.1.3.

1.2 In the even case, the matrix $B_{1}$ is self-adjoint if and only if (2.38) holds (cp. again $[3])$.

With $J_{1}$ chosen according to $(2.34)$, we obtain $V_{c}:=V_{c, 1}:=\operatorname{codiag}(i, \ldots, i,-i, \ldots,-i)$, with eigenvectors $e_{j}-i e_{2 m+1-j}$, which are associated with the eigenvalue -1 for $j \leq m$, and with the eigenvalue +1 for $m<j \leq 2 m$. Thus we have $n^{+}=n^{-}=m$. Furthermore, with $E_{c}:=E_{c, 1}:=\operatorname{diag}(1, \ldots, 1)+\operatorname{codiag}(-i, \ldots,-i)$, the pair $\left(V_{c}, E_{c}\right)$ is suitable.

Unfortunately, it will turn out that this choice of a suitable pair cannot lead to a selfadjoint operator. For this reason we introduce the following matrix $E_{s}:=\operatorname{diag}(1, \ldots, 1)+$ $\operatorname{codiag}(0, \ldots, 0,1, \ldots, 1)$ as an alternative to $E_{c, 1}$. To assure that the pair $\left(V_{c}, E_{s}\right)$ is suitable, we pose the additional assumption $w>0$ on an interval $(\tilde{a}, \tilde{b}) \subset[a, b)$.

Obviously, $E_{s}$ is regular. Furthermore $P_{\lambda}(a)=\frac{1}{2} \Phi_{\lambda}^{*}(a) V_{c} \Phi_{\lambda}(a)=0$. (As we will see, $P_{\lambda}(a)=$ 0 is the crucial point for the resulting operator to be possibly self-adjoint.) It remains to show, that $\zeta \in \mathbb{C}^{n^{+}}$with

$$
C_{\lambda, V_{c}} \Phi_{\lambda} \zeta=0 \text { a.e. in }[a, b)
$$

implies $\zeta=0$.

So let $\zeta \in \mathbb{C}^{n^{+}}$such that $(2.39)$ holds. The relation $C_{\lambda, V_{c}} \succcurlyeq A_{1}$ yields

$$
A_{1} \Phi_{\lambda} \zeta=0 \text { a.e. in }[a, b) \text {. }
$$

Let $v$ the first component of $\Phi_{\lambda} \zeta$. Recall $A_{1}=\operatorname{diag}(w, 0, \ldots, 0)$. Therewith (2.40) implies $v w=0$ a.e. on $[a, b)$. By continuity, $v$ is zero on $(\tilde{a}, \tilde{b})$, the interval where we assumed $w$ to 
be positive.

Let $v_{j}$ the $j^{\text {th }}$ component of $\Phi_{\lambda} \zeta$ for $0 \leq j \leq 2 m-1$. Since $\Phi_{\lambda}$ solves $J_{1} \Phi_{\lambda}^{\prime}=\left(\lambda A_{1}+B_{1}\right) \Phi_{\lambda}$, which is equivalent to $\Phi_{\lambda}^{\prime}=\left(\lambda M_{A}^{e}+M_{B}^{e}\right) \Phi_{\lambda}$, we also have

$$
\left(\Phi_{\lambda} \zeta\right)^{\prime}=\left(\lambda M_{A}^{e}+M_{B}^{e}\right) \Phi_{\lambda} \zeta
$$

where for $\left(a_{j, k}\right)_{j, k=1, \ldots, m}:=\left(\lambda M_{A}^{e}+M_{B}^{e}\right)$ holds $a_{j, j+1} \neq 0$ for $j<2 m$ and $a_{j, k}=0$ for $k>j+1$. We already know $v_{1}=v=0$ on $(\tilde{a}, \tilde{b})$, hence also $v_{1}^{\prime}=0$ on $(\tilde{a}, \tilde{b})$. By induction, using (2.41), we obtain $v_{j}=0$ on $(\tilde{a}, \tilde{b})$ for all $j \leq 2 m$. Thus $\Phi_{\lambda} \zeta=0$ on $(\tilde{a}, \tilde{b})$. By regularity of the differential equation $J_{1} Y^{\prime}=\left(\lambda A_{1}+B_{1}\right) Y$, of which $\Phi_{\lambda} \zeta$ is a solution, we obtain $\zeta=0$.

Hence $\left(V_{c}, E_{s}\right)$ is suitable. As we will see, this choice can lead to a self-adjoint operator.

1.3 For $B_{1}$ to be self-adjoint in the even case, we required $\bar{r}_{j}=-q_{j}$.

Yet now, besides $p_{j} \in \mathbb{R}$, we assume $\bar{r}_{j}=+q_{j}$ (for all $j \leq m$ ).

To obtain a self-adjoint $B$, we consider an alternative choice for $J$, namely

$$
J_{2}:=\operatorname{codiag}(1,-1,1,-1, \ldots, 1,-1) .
$$

Straightforward calculation shows $A_{2}:=J_{2} M_{A}^{e}=A_{1}$, furthermore that $B_{2}:=J_{2} M_{B}^{e}$ is selfadjoint. Thus, in this case, we could canonically choose $V= \pm i J_{2}$. Again we fix $V_{c, 2}:=+i J_{2}$. We obtain $n^{+}=n^{-}=m$.

The construction $E_{c, 2}:=\operatorname{diag}(1, \ldots, 1)+\operatorname{codiag}\left(-i, i, \ldots,(-1)^{m} i,(-1)^{m} i, \ldots, i,-i\right)$ yields the suitable pair $\left(V_{c, 2}, E_{c, 2}\right)$, where $\operatorname{Adm}_{V_{c, 2}}+\Lambda_{V_{c, 2}}$ is the lower halfplane.

Now we return to the original choice (2.34) of $J$. The calculations

$$
\begin{gathered}
C_{k, V_{c, 2}}=-\mathbf{R e}\left(V_{c, 2} J_{1}\left(k A_{1}+B_{1}\right)\right)=-\mathbf{R e}\left(V_{c, 2}\left(k M_{A}^{e}+M_{B}^{e}\right)\right)=-\mathbf{R e}\left(V_{c, 2} J_{2}\left(k A_{2}+B_{2}\right)\right), \\
-\mathbf{R e}\left(\lambda V_{c, 2} J_{1} A_{1}\right)=-\mathbf{R e}\left(\lambda V_{c, 2} M_{A}^{e}\right)=-\mathbf{R e}\left(\lambda V_{c, 2} J_{2} A_{2}\right)
\end{gathered}
$$

show that $\left(V_{c, 2}, E_{c, 2}\right)$ is still suitable and $\operatorname{Adm}_{V_{c, 2}}+\Lambda_{V_{c, 2}}$ is the lower halfplane. The actual choice of $J$ does not influent these properties.

Other assumptions on the coefficient functions $p_{j}, q_{j}$ and $r_{j}$ may be possible, under which we obtain the self-adjoint case for some choice of $J$.

2. We want to go into more detail in the odd-order case for $m=1$. So we have

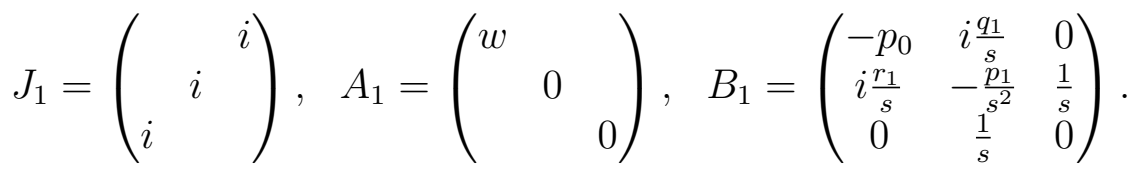


We examine the case that $s$ is real, furthermore the case that $e^{\frac{\pi}{4} i} s$ is real on $[a, b)$.

2.1 So first we assume that the highest order coefficient $s$ is real valued.

The requirement $-\boldsymbol{R e}(\lambda V J A) \succcurlyeq A$ for some $\lambda \in \mathbb{C}$ implies $V_{23}=V_{33}=V_{32}=0$ as a necessary condition. Hence $-\boldsymbol{R e}(V J(\lambda A+B)) \succcurlyeq 0$ yields further, by straightforward calculation, that $V_{13}=V_{22} \in \mathbb{R}$ and $V_{21}=V_{12}=0$, and for

$$
V=\left(\begin{array}{ccc}
v_{0} & 0 & v \\
0 & v & 0 \\
v & 0 & 0
\end{array}\right),
$$

with $v_{0}, v \in \mathbb{R}$, finally that

(i) $\operatorname{Re} i v p_{1} \succcurlyeq 0$

(ii) $4 v^{2} \boldsymbol{R e}\left(-i \lambda w+i p_{0}\right) \boldsymbol{R e}\left(i p_{1}\right)-\left|v\left(q_{1}+\bar{r}_{1}\right)-i v_{0}\right|^{2} \succcurlyeq 0$.

It is easily seen that (2.45), together with $(i),(i i)$, is indeed also sufficient for admissibility. $\operatorname{Adm}_{V}$ is the set of all $\lambda \in \mathbb{C}$ such that $(i i)$ holds. $\Lambda_{V}$ is the set of all $\lambda \in \mathbb{C}$, such that $\boldsymbol{\operatorname { R e }}(-i \lambda v w) \succcurlyeq w$; this is the upper or the lower halfplane, since $v$ is real.

2.2 Now we assume that $e^{\frac{\pi}{4} i} s$ is real.

For an admissible $V$ again we obtain $V_{12}=V_{21}=V_{23}=V_{32}=V_{33}=0$. But now we have $i V_{13}=V_{22} \in \mathbb{R}$, and thus

$$
V=\left(\begin{array}{ccc}
v_{0} & 0 & -i v \\
0 & v & 0 \\
i v & 0 & 0
\end{array}\right)
$$

for some $v_{0}, v \in \mathbb{R}$.

Again there are further requirements, analogous to $(i)$ and $(i i)$ above. Now $\Lambda_{V}$ is either the left or the right complex half-plane.

If $s$ is real on $[a, b)$, we could achieve the required assumption of $e^{\frac{\pi}{4}} i s$ being real by just multiplying the original equation with $-i$ and setting $\tilde{s}:=e^{-\frac{\pi}{4} i} s$. In this way we obtain the result of two non-intersecting halfplanes for $\Lambda_{V}$ also by the previous example 2.1. Obviously multiplying by $-i$ just rotates the problem by this factor (because $\tau_{o}[y]=\lambda w y$ becomes $\left.-i \tau_{o}[y]=(-i \lambda) w y\right)$. One might conjecture that a corresponding rotation argument holds true also for multiplication by other factors than $-i$. But if we multiply with by, say, $e^{-\frac{\pi}{4} i}$ (and set $\tilde{s}:=e^{-\frac{\pi}{8} i} s$ ), we obtain $e^{\frac{\pi}{4} i} V_{13}=V_{22} \in \mathbb{R}$ as a necessary condition for admissibility, and finally that $\Lambda_{V}$ is one of two halfplanes, which do intersect (their relative rotation is $\frac{\pi}{2}$ ). 
3. In most generality, we study the even-order case for $m=1$. To simplify the formulas, we set $p:=p_{1}, q:=q_{1}$, and $r:=r_{1}$. Thus the scalar equation reads

$$
p_{0} v-\left(p v^{\prime}\right)^{\prime}+q v^{\prime}+(r v)^{\prime}=\lambda w v .
$$

The modeling leads to the Hamiltonian System $J_{1} Y^{\prime}=\left(\lambda A_{1}+B_{1}\right) Y$ with

$$
J_{1}=\left(\begin{array}{ll} 
& -1 \\
1 &
\end{array}\right), \quad A_{1}=\left(\begin{array}{ll}
w & \\
& 0
\end{array}\right), \quad B_{1}=\frac{1}{p}\left(\begin{array}{cc}
-q r-p p_{0} & -q \\
r & 1
\end{array}\right) .
$$

As we have already realized, the existence of some $\lambda \in \mathbb{C}$ such that $-\operatorname{Re} \lambda V J_{1} A_{1} \succcurlyeq A_{1}$, implies that $V$ has some zero-entries since the rank of $A$ is one. In this two-dimensional case we obtain $V_{22}=0$. Thus any admissible matrix is of the shape

$$
V=\left(\begin{array}{cc}
v_{0} & \bar{v} \\
v & 0
\end{array}\right)
$$

for some real $v_{0}$ and some non-zero $v \in \mathbb{C}$.

Therefore, $V$ has a negative determinant. Hence we obtain $n^{+}=n^{-}=1$, and furthermore

$$
C_{\lambda, V}=-\boldsymbol{R e}\left(V J_{1}\left(\lambda A_{1}+B_{1}\right)\right)=\frac{1}{2}\left(\begin{array}{cc}
2 \boldsymbol{R e}\left(\frac{v_{0} r}{p}+\bar{v}\left(\frac{q r}{p}+p_{0}\right)-\lambda \bar{v} w\right) & \frac{v_{0}}{p}+\bar{v}\left(\frac{q}{p}+\frac{\bar{r}}{\bar{p}}\right) \\
\frac{v_{0}}{\bar{p}}+v\left(\frac{\bar{q}}{\bar{p}}+\frac{r}{p}\right) & 2 \boldsymbol{R e} \frac{v}{p}
\end{array}\right)
$$

The requirement $C_{\lambda, V} \succcurlyeq 0$ is equivalent to

$$
\begin{aligned}
& \boldsymbol{\operatorname { R e }}\left(\frac{v_{0} r}{p}+\bar{v}\left(\frac{q r}{p}+p_{0}\right)-\lambda \bar{v} w\right) \succcurlyeq 0, \\
& \operatorname{Re} \frac{v}{p} \succcurlyeq 0, \text { and } \\
& 4 \boldsymbol{\operatorname { R e }} \frac{v}{p} \boldsymbol{\operatorname { R e }}\left(\frac{v_{0} r}{p}+\bar{v}\left(\frac{q r}{p}+p_{0}\right)-\lambda \bar{v} w\right) \geq\left|\frac{v_{0}}{p}+\bar{v}\left(\frac{q}{p}+\frac{\bar{r}}{\bar{p}}\right)\right|^{2} \text { a.e. on }[a, b) .
\end{aligned}
$$

$\operatorname{Adm}_{V}$ is the set of all $\lambda \in \mathbb{C}$ for which these inequalities hold. $\Lambda_{V}$ is the halfplane $\{\lambda \in \mathbb{C}: \operatorname{Re} \lambda \bar{v}<0\}$. Hence $V$ is admissible if and only if $\mathrm{Adm}_{V}$ is not empty.

In [5], the scalar equation (2.46) was considered with $q=r=0$ and modeled in the same way to achieve a Hamiltonian system. The choice $\mathcal{U}_{2 n}=\operatorname{diag}(-u, \bar{u})$ in [5], corresponds to $V=\mathcal{U}_{2 n} J_{1}=\operatorname{codiag}(\bar{u}, u)$. This is of the form (2.47) with $v_{0}=0$ and $v=\bar{u}$. With all these choices, (2.49) reads

$$
\boldsymbol{\operatorname { R e }} \frac{\bar{u}}{p} \succcurlyeq 0, \text { and } \boldsymbol{\operatorname { R e }}\left(u\left(p_{0}-\lambda w\right)\right) \succcurlyeq 0 .
$$

An equivalent condition is given in [5, page 425]. 


\section{Chapter 3}

\section{The adjoint problem}

In this chapter we introduce the adjoint problem.

In the previous chapter most definitions and conclusions were mainly transferred from [5] to a more general situation. However in this chapter, using some linear algebraic theory, we are able to omit any further assumptions on the corresponding functions in the adjoint setting (like they were posed in [5]).

\subsection{Some linear algebraic statements}

The theory in this section provides us with some general knowledge on matrices. There is no direct statement on operator theory. But the statements in this section are very useful for the following theory.

Lemma 3.1.1. Let $U \in \mathbb{C}^{n, n}$ with

$$
U^{*} A U \succcurlyeq A \text {. }
$$

Then also

$$
A \succcurlyeq U^{*} A U \text {. }
$$

The proof of this Lemma is essentially due to Stefan Kühnlein.

Proof. Let $\delta>0$ such that $U^{*} A(x) U \geq \delta A(x)$ for a.e. $x \in[a, b)$. W.l.o.g. this inequality holds for all $x \in[a, b)$ and furthermore $\delta \leq 1$. 
First we assume that $A(x)>0$ for all $x \in[a, b)$. Then (3.1) implies that $U$ is regular.

Now we set

$$
\varepsilon:=\frac{\delta^{n-1}}{|\operatorname{det} U|^{2}}>0
$$

and

$$
N:=A^{-1 / 2} U^{*} A U A^{-1 / 2}
$$

$N$ is selfadjoint with $|\operatorname{det} N|=|\operatorname{det} U|^{2}$. Let $\lambda_{1} \leq \lambda_{2} \leq \cdots \leq \lambda_{n}$ denote the eigenvalues of $N$. On $[a, b)$ holds:

$$
\begin{aligned}
U^{*} A U-\delta A \geq 0 & \Rightarrow N-\delta I_{n} \geq 0 \\
& \Rightarrow \lambda_{1} \geq \delta \\
& \Rightarrow \operatorname{det} N=\lambda_{1} \cdot \ldots \cdot \lambda_{n} \geq \delta^{n-1} \lambda_{n} \\
& \Rightarrow \lambda_{n} \leq \frac{|\operatorname{det} U|^{2}}{\delta^{n-1}}=\frac{1}{\varepsilon} \\
& \Rightarrow I_{n}-\varepsilon N \geq 0 \\
& \Rightarrow A-\varepsilon U^{*} A U \geq 0 .
\end{aligned}
$$

Before examining the more general cases, we consider some simple implications.

For $x \in[a, b)$ with (3.1) we know that $\operatorname{ker} U^{*} A U \subseteq \operatorname{ker} A$. On the other hand the finite dimension of the null-space of $U^{*} A U$ is not smaller than the dimension of the null-space of $A$. So we can conclude

$$
\operatorname{ker} U^{*} A U=\operatorname{ker} A \text {. }
$$

Thus for $\xi \in \operatorname{ker} A$ we have

$$
0=\xi^{*} U^{*} A U \xi=\left(A^{1 / 2} U \xi\right)^{*}\left(A^{1 / 2} U \xi\right),
$$

and hence

$$
U(\operatorname{ker} A) \subseteq \operatorname{ker} A^{1 / 2}=\operatorname{ker} A .
$$

Now we only assume that $U$ is regular.

Let $x \in[a, b)$. Since $A(x)$ is self-adjoint, there exists a unitary matrix $W$ such that

$$
W^{*} A(x) W=\left(\begin{array}{cc}
\tilde{A} & 0 \\
0 & 0
\end{array}\right)
$$


with $\tilde{A}$ regular. From the inclusion (3.5) we see that

$$
W^{*} U W=\left(\begin{array}{cc}
\tilde{U} & 0 \\
* & X
\end{array}\right)
$$

with regular matrices $\tilde{U}$ and $X$, where $\tilde{U}$ and $\tilde{A}$ are of the same size. Assumption (3.1) implies

$$
\tilde{U}^{*} \tilde{A} \tilde{U} \geq \delta \tilde{A}
$$

Since $\tilde{A}$ is regular and $\delta \leq 1$, we obtain

$$
\tilde{A} \geq \frac{\delta^{n-1}}{|\operatorname{det} \tilde{U}|^{2}} \tilde{U}^{*} \tilde{A} \tilde{U}
$$

which implies

$$
W^{*} A(x) W \geq \frac{\delta^{n-1}}{|\operatorname{det} \tilde{U}|^{2}} W^{*} U^{*} A(x) U W
$$

and thus

$$
A(x) \geq \frac{\delta^{n-1}}{|\operatorname{det} \tilde{U}|^{2}} U^{*} A(x) U
$$

Since $\tilde{U}$ may depend on $x$, we consider characteristic polynomials. For those holds:

$$
\mathrm{CP}(U)=\operatorname{CP}(\tilde{U}) \operatorname{CP}(X) \text {. }
$$

Thus, $\mathrm{CP}(X)$ is a normalized divisor of $\mathrm{CP}(U)$, of which there are only finitely many. Since further $\operatorname{det} X=(\mathrm{CP}(X))(0)$, the definition

$$
\varepsilon:=\frac{\delta^{n-1}}{|\operatorname{det} U|^{2}} \min \left\{|\operatorname{det} \Xi|^{2}: \Xi \in \mathbb{C}^{n, n}, \mathrm{CP}(\Xi) \mid \mathrm{CP}(U)\right\}>0
$$

makes sense. This definition is independent of the actual choice of $x \in[a, b)$. As $\operatorname{det} U=\operatorname{det} X \operatorname{det} \tilde{U}$, it holds

$$
\varepsilon \leq \frac{\delta^{n-1}}{|\operatorname{det} \tilde{U}|^{2}}
$$

So with (3.8), we have for all $x \in[a, b)$,

$$
A(x) \geq \varepsilon U^{*} A(x) U .
$$


The last case to consider is where $U$ is singular, which we treat for completeness although it does not occur later. We assume w.l.o.g.

$$
U=\left(\begin{array}{ll}
\tilde{U} & 0 \\
* & 0
\end{array}\right)
$$

with $\tilde{U}$ regular. (Otherwise this structure of $U$ can be achieved by a unitary basis transformation.)

From equations (3.4) and since $A$ is self-adjoint we see that

$$
A=\left(\begin{array}{cc}
\tilde{A} & 0 \\
0 & 0
\end{array}\right) \text {. }
$$

(3.1) implies

$$
\tilde{U}^{*} \tilde{A} \tilde{U} \succcurlyeq \tilde{A} \text {. }
$$

Let $\varepsilon$ as in the case where $U$ is regular, with $\tilde{U}$ instead of $U$. So we have

$$
\tilde{A} \geq \varepsilon \tilde{U}^{*} \tilde{A} \tilde{U}
$$

which yields

$$
A(x) \geq \varepsilon U^{*} A(x) U
$$

for every $x \in[a, b)$.

Lemma 3.1.2. Let $U \in \mathbb{C}^{n, n}$ be such that

$$
\boldsymbol{R e}(A U) \succcurlyeq A
$$

Then,

$$
U^{*} A U \succcurlyeq A \text {. }
$$

Proof. Let $\delta>0$ such that $\boldsymbol{\operatorname { R e }}(A(x) U) \geq \delta A(x)$ for a.e. $x \in[a, b)$. For these $x$ we can estimate

$$
\begin{aligned}
U^{*} A(x) U & =(U-\delta I+\delta I)^{*} A(x)(U-\delta I+\delta I) \\
& =(U-\delta I)^{*} A(x)(U-\delta I)+\delta U^{*} A(x)+\delta A(x) U-\delta^{2} A(x)-\delta^{2} A(x)+\delta^{2} A(x) \\
& =(U-\delta I)^{*} A(x)(U-\delta I)+2 \delta \mathbf{R e}(A(x) U)-\delta^{2} A(x) \\
& \geq 0+2 \delta^{2} A(x)-\delta^{2} A(x)=\delta^{2} A(x) .
\end{aligned}
$$

Thus $U^{*} A(x) U \geq \delta^{2} A(x)$ for a.e. $x \in[a, b)$. 
The following Theorem is significant for many simplifications in the subsequent analysis.

Theorem 3.1.3. Let $U \in \mathbb{C}^{n, n}$ regular and $\lambda \in \mathbb{C}$. For the statements

(i) $\boldsymbol{\operatorname { R e }}(\lambda U A) \succcurlyeq U A U^{*}$,

(ii) $\boldsymbol{\operatorname { R e }}\left(\bar{\lambda} U^{-1} A\right) \succcurlyeq A$,

(iii) $\operatorname{Re}(\lambda U A) \succcurlyeq A$,

(iv) $A \asymp U A U^{*}$,

(v) $\forall \mu \in \mathbb{C}: A \succcurlyeq \boldsymbol{R e}(\mu U A)$,

we have

$$
(i) \Leftrightarrow(i i) \Leftrightarrow(i i i) \Rightarrow(i v) \Rightarrow(v),
$$

where $F \asymp G: \Leftrightarrow(F \succcurlyeq G$ and $G \succcurlyeq F)$ for functions $F, G:[a, b) \rightarrow \mathbb{C}^{n, n}$.

Proof. First of all we remark that, for $F, G:[a, b) \rightarrow \mathbb{C}^{n, n}$ and $H \in \mathbb{C}^{n, n}$,

$$
F \succcurlyeq G \Rightarrow H F H^{*} \succcurlyeq H G H^{*} \text {. }
$$

For $\lambda=0$, each of the statements $(i),($ ii $)$, or $(i i i)$ implies $A(x)=0$ for a.e. $x \in[a, b)$, whence the Theorem is trivial. So we can assume that $\lambda \neq 0$.

With (3.10) we obtain statement $(i i)$ from $(i)$ by multiplication with $U^{-1}$ and $\left(U^{*}\right)^{-1}$ from left and right, respectively. The other direction follows in the same way, multiplying by $U$ and $U^{*}$. Thus, we have $(i) \Leftrightarrow(i i)$.

Now let $(i)$ and $(i i)$ hold. Since $\boldsymbol{\operatorname { R e }}\left(\bar{\lambda} U^{-1} A\right)=\boldsymbol{\operatorname { R e }}\left(A\left(\bar{\lambda} U^{-1}\right)^{*}\right)$, (ii) together with Lemma 3.1 .2 yields

$$
|\lambda|^{2} U^{-1} A\left(U^{-1}\right)^{*} \succcurlyeq A \text {. }
$$

Using Lemma 3.1.1 we obtain

$$
A \succcurlyeq U^{-1} A\left(U^{-1}\right)^{*}
$$

Multiplication of $U$ and $U^{*}$ from left and right, respectively, gives

$$
U A U^{*} \succcurlyeq A \text {. }
$$

Again Lemma 3.1.1 yields

$$
A \succcurlyeq U A U^{*}
$$


These two equations are statement $(i v)$. So we have shown $(i) \Rightarrow(i v)$.

Statement $(i)$ and (3.11) imply statement (iii). Thus, $(i) \Rightarrow(i i i)$.

Now let (iii) hold. With Lemma 3.1.2 we know that $U A U^{*} \succcurlyeq A$, whence Lemma 3.1.1 yields $A \succcurlyeq U A U^{*}$. Statement (iii) together with this relation implies $(i)$. Thus, $(i i i) \Rightarrow(i)$.

Finally let $(i v)$ hold, and let $\mu \in \mathbb{C}$. W.l.o.g. we can assume $|\mu|=1$ since $A \succcurlyeq 0$. From

$$
\begin{aligned}
0 & \preccurlyeq(\mu U-I) A(\mu U-I)^{*} \\
& =A+U A U^{*}-\mu U A-A(\mu U)^{*} \\
& =A+U A U^{*}-2 \operatorname{Re}(\mu U A)
\end{aligned}
$$

we can conclude

$$
\boldsymbol{R e}(\mu U A) \preccurlyeq \frac{1}{2} A+\frac{1}{2} U A U^{*} \asymp \frac{1}{2} A+\frac{1}{2} A=A,
$$

which is statement $(v)$.

We finally need one more technical

Lemma 3.1.4. Let $F \in \mathbb{C}^{m, m}$ self-adjoint and regular with exactly $p$ positive eigenvalues. Further let $H$ be a p-dimensional subspace of $\mathbb{C}^{m}$ with $x^{*} F x \geq 0$ for all $x \in H$. Then it holds

$$
\forall y \in H^{\perp}: \quad y^{*} F^{-1} y \leq 0 .
$$

Proof. We assume there exists $y \in H^{\perp}$ with $y^{*} F^{-1} y>0$ and lead this to a contradiction. For $z:=F^{-1} y$ holds

$$
z^{*} F z=y^{*} F^{-1} y>0 .
$$

For $x \in H$ we have

$$
x^{*} F z=x^{*} y=0 .
$$

Together with (3.13), this shows that $z \in \mathbb{C}^{m} \backslash H$. Thus, $H+\operatorname{span}(z)$ is $(p+1)$-dimensional. The min-max principle therefore yields the existence of some

$$
w=x+\kappa z \in H+\operatorname{span}(z),
$$

with $x \in H$ and $\kappa \in \mathbb{C}$, such that

$$
w^{*} F w<0 .
$$

Moreover with (3.13) and (3.14) we obtain

$$
w^{*} F w=(x+\kappa z)^{*} F(x+\kappa z)=x^{*} F x+|\kappa|^{2} z^{*} F z \geq 0,
$$

a contradiction. Thus, the Lemma follows. 


\subsection{A suitable pair $(\widehat{V}, \widehat{E})$ for the adjoint Problem}

To achieve a suitable initial condition for the adjoint fundamental system we use the matrix $J_{0}$ defined in (3.18) below. It is remarkable that, in the setting of [5], $J_{0}=J$.

Recall that we have already fixed a suitable pair $(V, E)$ whose existence we have assumed.

For the formal adjoint problem

$$
J z_{\lambda}^{\prime}(x)=\left(\bar{\lambda} A(x)+B^{*}(x)\right) z_{\lambda}(x),
$$

we define

$$
\widehat{V}:=J V^{-1} J=-J V^{-1} J^{*}
$$

and

$$
\widehat{E}:=-J\left(E^{*}\right)^{-1} J_{0}
$$

with the unitary matrix

$$
J_{0}:=\left(\begin{array}{cc}
0 & -I_{n^{-}} \\
I_{n^{+}} & 0
\end{array}\right)
$$

We will see that $(\widehat{V}, \widehat{E})$ is suitable for (3.15). First we observe from (3.16) that $\widehat{V}$ is selfadjoint with $n^{-}$positive and $n^{+}$negative eigenvalues since $-V^{-1}$ is. This means that in the adjoint problem, $n^{+}$and $n^{-}$have exchanged their roles.

Note that the adjoint problem for (3.15) is the original problem, $V=J \widehat{V}^{-1} J$ and $E=$ $J\left(\widehat{E}^{*}\right)^{-1} J_{0}^{*}$, where $-J_{0}^{*}$ is of the form (3.18) with exchanged roles of $n^{+}$and $n^{-}$.

Let $Z_{\lambda}$ the fundamental matrix for (3.15) with $Z(a)=\widehat{E}$, and let

$$
\left(\eta_{\lambda} \mid \chi_{\lambda}\right):=Z_{\lambda}
$$

with $\eta_{\lambda}:[a, b) \rightarrow \mathbb{C}^{n, n^{+}}$and $\chi_{\lambda}:[a, b) \rightarrow \mathbb{C}^{n, n^{-}}$.

Lemma 3.2.1. ${ }^{*_{1}}$

$$
Z=-J\left(Y^{-1}\right)^{*} J_{0} .
$$

Proof. The right-hand side of (3.20) fulfills the initial condition. Furthermore,

$$
\begin{aligned}
0 & =\left((J Y)^{*} J\left(Y^{-1}\right)^{*}\right)^{\prime} \\
& =\left(J Y^{\prime}\right)^{*} J\left(Y^{-1}\right)^{*}+(J Y)^{*}\left(J\left(Y^{-1}\right)^{*}\right)^{\prime} \\
& =((\lambda A+B) Y)^{*} J\left(Y^{-1}\right)^{*}+Y^{*} J^{*}\left(J\left(Y^{-1}\right)^{*}\right)^{\prime} \\
& =Y^{*}\left(\bar{\lambda} A+B^{*}\right) J\left(Y^{-1}\right)^{*}-Y^{*} J\left(J\left(Y^{-1}\right)^{*}\right)^{\prime},
\end{aligned}
$$


thus $J\left(J\left(Y^{-1}\right)^{*}\right)^{\prime}=\left(\bar{\lambda} A+B^{*}\right) J\left(Y^{-1}\right)^{*}$. This shows that the right-hand side of (3.20) also fulfills the differential equation. With the assumed regularity, the initial value problem is uniquely solvable, whence the statement holds.

Equivalent to this Lemma is

$$
Y^{*} J Z=J_{0}
$$

Taking the adjoint on both sides, and multiplying with $-J Y J_{0}$ from the left and with $(J Y)^{-1}$ from the right we obtain

$$
J Y J_{0} Z^{*}=-I_{n} .
$$

We will need these equations later on.

Now we want to proof admissibility of $\widehat{V}$, which means there exist $\lambda, k \in \mathbb{C}$ such that

$$
\widehat{C_{k}}:=-\operatorname{Re}\left(\widehat{V} J\left(\bar{k} A+B^{*}\right)\right) \succcurlyeq 0
$$

and

$$
-\boldsymbol{R e}(\bar{\lambda} \widehat{V} J A) \succcurlyeq A
$$

We show even more:

Theorem 3.2.2. ${ }^{* 4}$ Equations (3.24) and (3.25) are equivalent to (2.3) and (2.4), respectively, the corresponding equations for $V$.

Proof. Since $\boldsymbol{\operatorname { R e }}(F)=\boldsymbol{\operatorname { R e }}\left(F^{*}\right)$ and $\boldsymbol{\operatorname { R e }}\left(G^{*} F G\right)=G^{*} \boldsymbol{\operatorname { R e }}(F) G$ for any matrices $F$ and $G$, it holds

$$
\begin{aligned}
\widehat{C_{k}} & =-\boldsymbol{R e}\left((k A+B) J^{*} \widehat{V}\right) \\
& =-\boldsymbol{R e}\left((k A+B) V^{-1} J\right) \\
& =-\boldsymbol{R e}\left(\left(V^{-1} J\right)^{*} V J(k A+B) V^{-1} J\right) \\
& =\left(V^{-1} J\right)^{*} C_{k}\left(V^{-1} J\right) .
\end{aligned}
$$

$\left(V^{-1} J\right)$ is regular and so $\widehat{C_{k}} \succcurlyeq 0$ if and only if $C_{k} \succcurlyeq 0$.

The second equivalence follows from Theorem 3.1.3 (ii) $\Leftrightarrow$ (iii) with $U:=-V J$ (and hence $\left.U^{-1}=-\widehat{V} J\right)$.

An immediate consequence of this Theorem is 
Corollary 3.2.3. $\widehat{V}$ is admissible for the adjoint Problem and

$$
\begin{gathered}
\widehat{A d m_{\widehat{V}}}:=\left\{k \in \mathbb{C}: \widehat{C_{k}}(x) \succcurlyeq 0\right\}=A d m_{V}, \\
\widehat{\Lambda_{\widehat{V}}}:=\{\lambda \in \mathbb{C}:-\operatorname{Re}(\bar{\lambda} \widehat{V} J A) \succcurlyeq A\}=\Lambda_{V} .
\end{gathered}
$$

We define, in analogy to (2.18),

$$
\left(\begin{array}{cc}
\widehat{S_{\lambda}} & \widehat{T_{\lambda}} \\
{\widehat{T_{\lambda}}}^{*} & \widehat{P_{\lambda}}
\end{array}\right):=\frac{1}{2} Z_{\lambda}^{*} \widehat{V} Z_{\lambda},
$$

with $\widehat{P_{\lambda}} \in \mathbb{C}^{n^{-}, n^{-}}, \widehat{S_{\lambda}} \in \mathbb{C}^{n^{+}, n^{+}}$and $\widehat{T_{\lambda}} \in \mathbb{C}^{n^{+}, n^{-}}$.

Then,

$$
\widehat{P_{\lambda}}=\frac{1}{2} \chi_{\lambda}^{*} \widehat{V} \chi_{\lambda}
$$

which corresponds to (2.8).

\section{Lemma 3.2.4.}

$$
\widehat{P_{\lambda}}(a) \geq 0
$$

Proof. $P_{\lambda}(a) \geq 0$ and the definition of $P_{\lambda}$ yield

$$
\forall x \in \operatorname{span}\left\{e_{n^{-}+1}, \ldots, e_{n}\right\}: x^{*} E^{*} V E x \geq 0,
$$

with $e_{j}:=(\underbrace{0, \ldots, 0}_{j-1}, 1,0, \ldots, 0)^{T}$. Since $E^{*} V E$ has exactly $n^{+}=n-n^{-}$positive eigenvalues, Lemma 3.1.4 implies

$$
\forall y \in \operatorname{span}\left\{e_{1}, \ldots, e_{n^{-}}\right\}: y^{*}\left(E^{*} V E\right)^{-1} y \leq 0 .
$$

Furthermore, by definition,

$$
\begin{aligned}
2\left(\begin{array}{cc}
\widehat{S_{\lambda}} & \widehat{T_{\lambda}} \\
\widehat{T_{\lambda}} & \widehat{P_{\lambda}}
\end{array}\right)(a) & =\widehat{E}^{*} \widehat{V} \widehat{E} \\
& =\left(J\left(E^{*}\right)^{-1} J_{0}\right)^{*} J V^{-1} J J\left(E^{*}\right)^{-1} J_{0} \\
& =-J_{0}{ }^{*} E^{-1} V^{-1}\left(E^{*}\right)^{-1} J_{0} \\
& =-J_{0}{ }^{*}\left(E^{*} V E\right)^{-1} J_{0} .
\end{aligned}
$$

By the form of $J_{0}$, for $x \in \operatorname{span}\left\{e_{n^{+}+1}, \ldots, e_{n}\right\}$ we have $J_{0} x \in \operatorname{span}\left\{e_{1}, \ldots, e_{n^{-}}\right\}$. Thus (3.30) yields

$$
\forall x \in \operatorname{span}\left\{e_{n^{+}+1}, \ldots, e_{n}\right\}:-x^{*} J_{0}{ }^{*}\left(E^{*} V E\right)^{-1} J_{0} x \geq 0,
$$

which means $\widehat{P_{\lambda}}(a) \geq 0$ by (3.31). 
The last statement to prove for $(\widehat{V}, \widehat{E})$ to be suitable for the adjoint problem is

$$
\left(\widehat{P_{\lambda}}(a) \zeta=0 \text { and }\left(\widehat{C_{\lambda}} \chi_{\lambda}\right)(x) \zeta=0 \text { for a.e. } \mathrm{x} \in[a, b)\right) \Rightarrow \zeta=0,
$$

for all $\zeta \in \mathbb{C}^{n^{-}}$and $\lambda \in \operatorname{Adm}_{V}+\Lambda_{V}$.

Theorem 3.2.5.*2 For $\lambda \in \mathbb{C},(2.10)$ and (3.32) are equivalent.

Proof. By symmetry it suffices to show that (3.32) implies (2.10). So let (3.32) hold and let $\zeta \in \mathbb{C}^{n^{+}}$satisfy the left-hand side of (2.10).

By assumption, a.e. on $[a, b)$,

$$
0=2 C_{\lambda} \Phi_{\lambda} \zeta=-2 \operatorname{Re}(V J(\lambda A+B)) \Phi_{\lambda} \zeta=\left(\bar{\lambda} A+B^{*}\right) J V \Phi_{\lambda} \zeta-V J(\lambda A+B) \Phi_{\lambda} \zeta,
$$

and hence

$$
J\left(J V \Phi_{\lambda} \zeta\right)^{\prime}=-V \Phi_{\lambda}^{\prime} \zeta=V J\left(J \Phi_{\lambda}^{\prime}\right) \zeta=V J(\lambda A+B) \Phi_{\lambda} \zeta=\left(\bar{\lambda} A+B^{*}\right) J V \Phi_{\lambda} \zeta .
$$

This shows that $J V \Phi_{\lambda} \zeta$ solves the adjoint problem (3.15). Thus there exist $\xi_{1} \in \mathbb{C}^{n^{+}}, \xi_{2} \in \mathbb{C}^{n^{-}}$ such that

$$
J V \Phi_{\lambda} \zeta=Z_{\lambda}\left(\begin{array}{l}
\xi_{1} \\
\xi_{2}
\end{array}\right) .
$$

Using (2.18) and (3.22) we have (on $[a, b))$

$$
2\left(\begin{array}{c}
T_{\lambda} \\
P_{\lambda}
\end{array}\right) \zeta=Y_{\lambda}^{*} V \Phi_{\lambda} \zeta=-Y_{\lambda}^{*} J J V \Phi_{\lambda} \zeta=-Y_{\lambda}^{*} J Z_{\lambda}\left(\begin{array}{l}
\xi_{1} \\
\xi_{2}
\end{array}\right)=-J_{0}\left(\begin{array}{l}
\xi_{1} \\
\xi_{2}
\end{array}\right)=\left(\begin{array}{c}
\xi_{2} \\
-\xi_{1}
\end{array}\right) .
$$

$P_{\lambda}(a) \zeta=0$ yields $\xi_{1}=0$, and thus (3.33) reads

$$
J V \Phi_{\lambda} \zeta=\chi_{\lambda} \xi_{2}
$$

Since $C_{\lambda} \Phi_{\lambda} \zeta=0$ for a.e. $x \in[a, b)$, we obtain with $(3.26)$

$$
\widehat{C_{\lambda}} \chi_{\lambda} \xi_{2}=0 \quad \text { for a.e. } x \in[a, b) \text {. }
$$

Equation (3.20) implies $Z_{\lambda} J_{0}^{*}=-J\left(Y_{\lambda}^{-1}\right)^{*}$ and thus

$$
J Z_{\lambda} J_{0}^{*} Y_{\lambda}^{*}=I_{n}
$$


Furthermore (3.16) gives

$$
\widehat{V} J V J=I_{n} .
$$

With the equations above and (3.22) we obtain

$$
\begin{aligned}
J_{0}^{*} & =Z_{\lambda}^{*} J^{*} Y_{\lambda} \\
& =Z_{\lambda}^{*}\left(\widehat{V} J\left(J Z_{\lambda} J_{0}^{*} Y_{\lambda}^{*}\right) V J\right) J^{*} Y_{\lambda} \\
& =-\left(Z_{\lambda}^{*} \widehat{V} Z_{\lambda}\right) J_{0}^{*}\left(Y_{\lambda}^{*} V Y_{\lambda}\right) \\
& =-4\left(\begin{array}{cc}
\widehat{S_{\lambda}} & \widehat{T_{\lambda}} \\
{\widehat{T_{\lambda}}}^{*} & \widehat{P_{\lambda}}
\end{array}\right) J_{0}^{*}\left(\begin{array}{ll}
S_{\lambda} & T_{\lambda} \\
T_{\lambda}^{*} & P_{\lambda}
\end{array}\right) .
\end{aligned}
$$

Let $\widehat{N}^{+} \in \mathbb{C}^{n^{+}, n^{+}}$and $\widehat{N}^{-} \in \mathbb{C}^{n^{-}, n^{+}}$(cp. Lemma 2.2.6) such that

$$
\left(\begin{array}{cc}
\widehat{S_{\lambda}} & \widehat{T_{\lambda}} \\
{\widehat{T_{\lambda}}}^{*} & \widehat{P_{\lambda}}
\end{array}\right)\left(\begin{array}{l}
\widehat{N}^{+} \\
\widehat{N}^{-}
\end{array}\right)=\left(\begin{array}{c}
I_{n^{+}} \\
0
\end{array}\right) .
$$

This yields

$$
\widehat{P_{\lambda}} \widehat{N}^{-}=-\widehat{T}_{\lambda}^{*} \widehat{N}^{+}
$$

With (3.37) we obtain

$$
\begin{aligned}
\left(\begin{array}{c}
\widehat{N}^{-} \\
-\widehat{N}^{+}
\end{array}\right) & =-J_{0}\left(\begin{array}{l}
\widehat{N}^{+} \\
\widehat{N}^{-}
\end{array}\right) \\
& =4\left(\begin{array}{ll}
S_{\lambda} & T_{\lambda} \\
T_{\lambda}^{*} & P_{\lambda}
\end{array}\right) J_{0}\left(\begin{array}{cc}
\widehat{S_{\lambda}} & \widehat{T_{\lambda}} \\
\widehat{T}_{\lambda}^{*} & \widehat{P_{\lambda}}
\end{array}\right)\left(\begin{array}{c}
\widehat{N}^{+} \\
\widehat{N}^{-}
\end{array}\right) \\
& =4\left(\begin{array}{ll}
S_{\lambda} & T_{\lambda} \\
T_{\lambda}^{*} & P_{\lambda}
\end{array}\right) J_{0}\left(\begin{array}{c}
I_{n^{+}} \\
0
\end{array}\right) \\
& =4\left(\begin{array}{ll}
S_{\lambda} & T_{\lambda} \\
T_{\lambda}^{*} & P_{\lambda}
\end{array}\right)\left(\begin{array}{c}
0 \\
I_{n^{+}}
\end{array}\right) \\
& =4\left(\begin{array}{l}
T_{\lambda} \\
P_{\lambda}
\end{array}\right)
\end{aligned}
$$

Using (3.39) we obtain

$$
\widehat{P_{\lambda}} T_{\lambda}={\widehat{T_{\lambda}}}^{*} P_{\lambda}
$$

Thus (3.34) yields

$$
\widehat{P_{\lambda}}(a) \xi_{2}=2 \widehat{P_{\lambda}}(a) T_{\lambda}(a) \zeta=2 \widehat{T_{\lambda}}(a)^{*} P_{\lambda}(a) \zeta=0 .
$$

(3.36) and (3.42) is the premise of (3.32) for $\xi_{2}$, whence $\xi_{2}=0$ by assumption. Consequently $J V \Phi_{\lambda} \zeta=0$ by (3.35), implying $\zeta=0$ since $J V \Phi_{\lambda}$ has rank $n^{+}$. 
Now, all required estimates are proven for

Corollary 3.2.6. $(\widehat{V}, \widehat{E})$ is suitable for the adjoint problem.

\subsection{Weyl-Sims circles in the adjoint setting}

We define for $\lambda \in \operatorname{Adm}_{V}+\Lambda_{V}$, in analogy to section 2.2,

$$
\widehat{D_{\lambda}}(x):=\left\{l \in \mathbb{C}^{n^{-}, n^{+}}:\left[\left(\eta_{\lambda}+\chi_{\lambda} l\right)^{*} \widehat{V}\left(\eta_{\lambda}+\chi_{\lambda} l\right)\right](x) \leq 0\right\},
$$

and for $x$ large enough

$$
\begin{gathered}
\widehat{\mathcal{C}_{\lambda}}:=-{\widehat{P_{\lambda}}}^{-1}{\widehat{T_{\lambda}}}^{*}, \\
\widehat{\mathcal{R}_{\lambda}}:={\widehat{T_{\lambda}}}^{-1}{\widehat{T_{\lambda}}}^{*}-\widehat{S_{\lambda}} .
\end{gathered}
$$

By symmetry all statements of section 2.2 hold correspondingly.

We can assume w.l.o.g. that for $\lambda \in \operatorname{Adm}_{V}+\Lambda_{V}$ and $x \geq c_{0}(\lambda)$ also $\widehat{P_{\lambda}}(x)>0$.

Let $\lambda \in \operatorname{Adm}_{V}+\Lambda_{V}$. Using (3.41) and (2.30), we obtain, on $\left[c_{0}(\lambda), b\right)$,

$$
\begin{gathered}
\widehat{\mathcal{C}_{\lambda}}=-{\widehat{P_{\lambda}}}^{-1}{\widehat{T_{\lambda}}}^{*}=-{\widehat{P_{\lambda}}}^{-1}{\widehat{T_{\lambda}}}^{*} P_{\lambda} P_{\lambda}^{-1}=-T_{\lambda} P_{\lambda}^{-1}=\mathcal{C}_{\lambda}^{*}, \\
\mathcal{R}_{\lambda}=\frac{1}{4}{\widehat{P_{\lambda}}}^{-1}
\end{gathered}
$$

and by symmetry

$$
\widehat{\mathcal{R}_{\lambda}}=\frac{1}{4} P_{\lambda}^{-1}
$$

Therewith we can determine the connection between the Weyl-Sims sets of the original problem and those of the adjoint problem.

Lemma 3.3.1. ${ }^{*}$ For $\lambda \in A d m_{V}+\Lambda_{V}$ and $x \geq c_{0}(\lambda)$ it holds

$$
\widehat{D_{\lambda}}(x)=D_{\lambda}^{*}(x)
$$


Proof. We use the notation (2.27) for $D_{\lambda}(x)$. The corresponding "adjoint" statement reads

$$
\widehat{D_{\lambda}}(x)=\widehat{\mathcal{C}_{\lambda}}(x)+\left\{{\widehat{P_{\lambda}}}^{-1 / 2}(x) N{\widehat{\mathcal{R}_{\lambda}}}^{1 / 2}(x): N \in \mathbb{C}^{n^{-}, n^{+}} \text {with } N^{*} N \leq I_{n^{+}}\right\}
$$

Let $\mathcal{C}_{\lambda}(x)+P_{\lambda}^{-1 / 2}(x) N \mathcal{R}_{\lambda}^{1 / 2}(x) \in D_{\lambda}(x)$ with $N \in \mathbb{C}^{n^{+}, n^{-}}, N^{*} N \leq I_{n^{-}}$.

The last property means that the norm of $N$ as an operator $\left(\mathbb{C}^{n^{-}},\|\cdot\|_{2}\right) \rightarrow\left(\mathbb{C}^{n^{+}},\|\cdot\|_{2}\right)$ is not bigger than 1 . This is equivalent to the corresponding statement for $N^{*}$. Thus, by $(3.49)$,

$$
\widehat{\mathcal{C}_{\lambda}}(x)+{\widehat{P_{\lambda}}}^{-1 / 2}(x) N^{*}{\widehat{\mathcal{R}_{\lambda}}}^{1 / 2}(x) \in \widehat{D_{\lambda}}(x)
$$

Since $P_{\lambda}(\mathrm{x})$ and $\mathcal{R}_{\lambda}(x)$ are self-adjoint, (3.47) and (3.48) yield

$$
P_{\lambda}^{-1 / 2}(x) N \mathcal{R}_{\lambda}^{1 / 2}(x)=\widehat{\mathcal{R}}_{\lambda}^{1 / 2}(x) N{\widehat{P_{\lambda}}}^{-1 / 2}(x)=\left({\widehat{P}_{\lambda}}^{-1 / 2}(x) N^{*} \widehat{\mathcal{R}}_{\lambda}^{1 / 2}(x)\right)^{*} .
$$

With equation (3.46), and symmetry, the assertion follows.

\subsection{Smoothness of $\lambda \mapsto Y_{\lambda}$}

We will show pointwise (in $x$ ) analyticity of $Y_{\lambda}$ with respect to $\lambda$, furthermore continuity thereof within $L_{A}^{2}$. The author presumes that this is a statement known already, but could not find a suitable direct reference. We will use it further below, to show some spectral estimates.

First we need the following

Lemma 3.4.1. ${ }^{*}$ For $\lambda_{0}, \lambda \in \mathbb{C}, c \in[a, b)$, the problem

$$
J y^{\prime}=(\lambda A+B) y
$$

is equivalent to the following problem:

There exists some $\xi \in \mathbb{C}^{n}$ such that, for all $x \in[a, b)$,

$$
y(x)=Y_{\lambda_{0}}(x) \xi-\left(\lambda-\lambda_{0}\right) Y_{\lambda_{0}}(x) J_{0} \int_{c}^{x} Z_{\lambda_{0}}^{*}(t) A(t) y(t) d t .
$$


Proof. Since the solution-space of (3.50) has dimension $n$, it suffices to show that any solution of (3.51) solves (3.50).

So let $y$ a solution of (3.51). From (3.23) we see that $y$ solves

$$
y(x)=Y_{\lambda_{0}}(x) \xi-\left(\lambda-\lambda_{0}\right) Y_{\lambda_{0}}(x) \int_{c}^{x} Y_{\lambda_{0}}^{-1}(t) J A(t) y(t) d t .
$$

Pre-multiplication with $J$, differentiation, and use of $J J=-I_{n}$ shows that $y$ solves

$$
J y^{\prime}=\left(\lambda_{0} A+B\right) y+\left(\lambda-\lambda_{0}\right) A y=(\lambda A+B) y .
$$

Now we are able to prove

Theorem 3.4.2. (i) For fixed $x \in[a, b)$, the function $\lambda \mapsto Y_{\lambda}(x)$ is (componentwise) analytic.

(ii) If all solutions of (2.1) and all solutions of (3.15) are in $L_{A}^{2}$ for all $\lambda \in \mathbb{C}$, then the mapping

$$
\mathbb{C} \rightarrow \mathbb{R}, \quad \lambda \mapsto\left\|\left(Y_{\lambda}\right)_{j}\right\|_{L_{A}^{2}}
$$

is continuous for any $j \leq n$, where $\left(Y_{\lambda}\right)_{j}$ denotes the $j$-th column of $Y_{\lambda}$.

(For the proper definition of $L_{A}^{2}$ see section 4.1 below.)

Proof. The main idea of the proof of $(i)$ is due to Michael Plum.

Let $\lambda_{0} \in \mathbb{C}$ and $c:=a$ in view of Lemma 3.4.1. For every $\lambda \in \mathbb{C}$, equation (3.51) implies

$$
Y_{\lambda}(x)=Y_{\lambda_{0}}(x) \Xi-\left(\lambda-\lambda_{0}\right) Y_{\lambda_{0}}(x) J_{0} \int_{a}^{x} Z_{\lambda_{0}}^{*}(s) A(s) Y_{\lambda}(s) d s
$$

Evaluation at $a$ yields $\Xi=I_{n}$. Now we pick an arbitrary column of $Y_{\lambda}$, which we call $y_{\lambda}$. Let $y_{\lambda_{0}}$ the corresponding column of $Y_{\lambda_{0}}$. For statement $(i)$ it suffices to show analyticity of $y_{\lambda}$. We already know

$$
y_{\lambda}(x)=y_{\lambda_{0}}(x)-\left(\lambda-\lambda_{0}\right) Y_{\lambda_{0}}(x) J_{0} \int_{a}^{x} Z_{\lambda_{0}}^{*}(s) A(s) y_{\lambda}(s) d s .
$$

Let $d \in(a, b)$. We define the operator

$$
\begin{aligned}
& F: L_{A}^{2}(a, d) \rightarrow L_{A}^{2}(a, d) \\
& (F z)(x):=Y_{\lambda_{0}}(x) J_{0} \int_{a}^{x} Z_{\lambda_{0}}^{*}(s) A(s) z(s) d s .
\end{aligned}
$$


Now we show that $F$ is well-defined and bounded:

Therefor we use the abbreviations $\Omega:=Y_{\lambda_{0}} J_{0},\|\cdot\|_{x}:=\|\cdot\|_{A,(a, x)}$, and $\xi_{j}$ for the $j$-th row of $Z_{\lambda_{0}}^{*}$. For $z \in L_{A}^{2}(a, d)$ we can estimate

$$
\begin{aligned}
\|F z\|_{d}^{2} & =\int_{a}^{d} \int_{a}^{x} z^{*}(s) A(s) Z_{\lambda_{0}}(s) d s \quad \Omega^{*}(x) A \Omega(x) \quad \int_{a}^{x} Z_{\lambda_{0}}^{*}(s) A(s) z(s) d s d x \\
& \leq \int_{a}^{d}\left|\int_{a}^{x} z^{*}(s) A(s) Z_{\lambda_{0}}(s) d s\right|_{2}\left|\Omega^{*}(x) A \Omega(x)\right|_{2}\left|\int_{a}^{x} Z_{\lambda_{0}}^{*}(s) A(s) z(s) d s\right|_{2} d x \\
(3.54) & =\int_{a}^{d}\left|\Omega^{*}(x) A \Omega(x)\right|_{2}\left|\int_{a}^{x} Z_{\lambda_{0}}^{*}(s) A(s) z(s) d s\right|_{2}^{2} d x \\
& =\sum_{j=1}^{n} \int_{a}^{d}\left|\Omega^{*}(x) A \Omega(x)\right|_{2}\left(\int_{a}^{x} \xi_{j}(s) A(s) z(s) d s\right)^{2} d x \\
& \leq \sum_{j=1}^{n} \int_{a}^{d}\left|\Omega^{*}(x) A \Omega(x)\right|_{2}\left\|\xi_{j}\right\|_{x}^{2}\|z\|_{x}^{2} d x \\
& \leq \sum_{j=1}^{n} \int_{a}^{d}\left|\Omega^{*}(x) A \Omega(x)\right|_{2}\left\|\xi_{j}\right\|_{d}^{2}\|z\|_{d}^{2} d x \leq\|z\|_{d}^{2}\left(\sum_{j=1}^{n}\left\|\xi_{j}\right\|_{d}^{2} \int_{a}^{d}\left|\Omega^{*}(x) A \Omega(x)\right|_{2} d x\right) .
\end{aligned}
$$

Using (3.52) we know that $y_{\lambda}$ satisfies

$$
y_{\lambda}=y_{\lambda_{0}}-\left(\lambda-\lambda_{0}\right) F y_{\lambda}
$$

This is equivalent to

$$
\left(I_{n}-\left(\lambda_{0}-\lambda\right) F\right) y_{\lambda}=y_{\lambda_{0}}
$$

Let $\delta:=\frac{1}{2\|F\|}$. For $\lambda \in \mathbb{C}$ with $\left|\lambda_{0}-\lambda\right| \leq \delta$ there exists exactly one function in $L_{A}^{2}(a, d)$ solving (3.55). So this equation uniquely characterizes $y_{\lambda}$, which is given by the Neumann series:

$$
y_{\lambda}=\sum_{j=0}^{\infty}\left(\lambda_{0}-\lambda\right)^{j} F^{j} y_{\lambda_{0}} .
$$

The series converges in $L_{A}^{2}(a, d)$. But it also converges uniformly, i.e. in $\left(C[a, d]^{n},\|\cdot\|_{\infty}\right)$ : For $j \in \mathbb{N}$ we have

$$
\left\|\left(\lambda_{0}-\lambda\right)^{j} F^{j} y_{\lambda_{0}}\right\|_{L_{A}^{2}(a, d)} \leq 2^{-j}\left\|y_{\lambda_{0}}\right\|_{L_{A}^{2}(a, d)},
$$

by assumption on $\delta$. By definition of $F$, the Cauchy-Schwarz inequality yields

$$
\|F z\|_{\infty,[a, d]} \leq K\|z\|_{A,(a, d)} \quad \text { for all } z \in L_{A}^{2}(a, d),
$$


with a constant $\mathrm{K}$, only depending on $Y_{\lambda_{0}}$ and $\left\|Z_{\lambda_{0}}\right\|_{L_{A}^{2}(a, d)}$. Thus, (3.57) gives

$$
\left\|\left(\lambda-\lambda_{0}\right)^{j+1} F^{j+1} y_{\lambda_{0}}\right\|_{\infty,[a, d]} \leq \widetilde{K} 2^{-j}
$$

proving the desired $\|\cdot\|_{\infty}$-convergence of the series (3.56).

Since $\|\cdot\|_{\infty}$-convergence implies convergence in $L_{A}^{2}(a, d)$, the limit coincides with $y_{\lambda}$.

With $\|F\|=\frac{1}{2 \delta}$, the Neumann series shows that, for each fixed $x, \lambda \mapsto y_{\lambda}(x)$ is analytic on $\left\{\lambda \in \mathbb{C}:\left|\lambda-\lambda_{0}\right|<\delta\right\}$ and therefore analytic on $\mathbb{C}$, since $\lambda_{0}$ is arbitrary.

For proving $(i i)$ we choose again some $\lambda_{0} \in \mathbb{C}$. If we set $y_{\lambda}:=\left(Y_{\lambda}\right)_{j}$, we obtain $(3.52)$ again.

With the stronger assumption made now we can choose $d:=b$ to define the operator $F$ : $L_{A}^{2} \rightarrow L_{A}^{2}$ via (3.53). The estimation (3.54) still holds, implying that $F$ is bounded with $\|F\|_{L_{A}^{2}}<Q$ for some constant $Q$ depending on $\lambda_{0}$ but not on $\lambda$.

For all $\lambda \in \mathbb{C}$ with $\left|\lambda_{0}-\lambda\right|<\frac{1}{2 Q}$, equation (3.56) yields

$$
\left\|y_{\lambda}-y_{\lambda_{0}}\right\|_{L_{A}^{2}} \leq \sum_{j=1}^{\infty}\left\|\left(\lambda_{0}-\lambda\right)^{j} F^{j} y_{\lambda_{0}}\right\|_{L_{A}^{2}} \leq \sum_{j=1}^{\infty}\left|\lambda_{0}-\lambda\right|^{j} Q^{j}\left\|y_{\lambda_{0}}\right\|_{L_{A}^{2}} .
$$

The right-hand side tends to 0 as $\lambda$ tends to $\lambda_{0}$. Hence statement $(i i)$ follows. 


\section{Chapter 4}

\section{A differential operator}

In this chapter we define an $M$-function, operators $R_{\lambda}$ and $\widehat{R}_{\lambda}$, and, with one further assumption, differential operators ( $L$ for the original problem and $\widehat{L}$ for the adjoint problem). It turns out that $L^{*}=\widehat{L}$ and $R_{\lambda}=(L-\lambda)^{-1}$ for $\lambda \in \operatorname{Adm}_{V}+\Lambda_{V}$.

Finally, we define the maximal operator for (2.1) and examine the deficiency indices.

\subsection{Hilbert spaces}

Definition 4.1.1. Let $\tilde{a}, \tilde{b} \in[a, b) \cup\{b\}$ with $\tilde{a}<\tilde{b}$. For $F:[\tilde{a}, \tilde{b}) \rightarrow \mathbb{C}^{n, n}$ such that $F(x)$ is self-adjoint and $F(x) \geq 0$ for a.e. $x \in[\tilde{a}, \tilde{b})$, let $L_{F}^{2}(\tilde{a}, \tilde{b})$ denote the Hilbert space of (equivalence classes of) measurable functions $f:[\tilde{a}, \tilde{b}) \rightarrow \mathbb{C}^{n}$ such that

$$
\|f\|_{F}^{2}:=\int_{\tilde{a}}^{\tilde{b}} f^{*} F f d x<\infty,
$$

where functions $f, g$ are regarded as equivalent if their difference lies in the null-space of $F$ almost everywhere, which means $\|f-g\|_{F}=0$.

We further use the abbreviation $L_{F}^{2}$ for $L_{F}^{2}(a, b)$.

Thus for such $F$ and $G$ we have

$$
F \succcurlyeq G \Rightarrow L_{F}^{2} \subseteq L_{G}^{2}
$$

and

$$
F \succcurlyeq G \Rightarrow\|f\|_{F} \geq \delta\|f\|_{G}
$$

for some $\delta>0$ and all $f \in L_{F}^{2}$. 
Besides $L_{A}^{2}$, also $L_{C_{\lambda}}^{2}$ and $L_{\widehat{C_{\lambda}}}^{2}$ occur in the following theory. In this section we give some estimates showing relations between these spaces.

For $U=-V J$ statement (iii) in Theorem 3.1.3 is equivalent to $\lambda \in \Lambda_{V}$. With (3.16) we obtain

Corollary 4.1.2. For $\nu \in \Lambda_{V}$ and $\mu \in \mathbb{C}$,

(i) $-\operatorname{Re}(\nu V J A) \succcurlyeq V J A J^{*} V$

(ii) $-\boldsymbol{\operatorname { R e }}(\bar{\nu} \widehat{V} J A) \succcurlyeq A$,

(iii) $-\boldsymbol{\operatorname { R e }}(\nu V J A) \succcurlyeq A$,

(iv) $A \asymp V J A J^{*} V$,

(v) $A \succcurlyeq \boldsymbol{R e}(\mu V J A)$.

This Corollary is essential for many conclusions in the further examination.

Corollary 2.2.1 states $C_{\lambda} \succcurlyeq A$ for $\lambda \in \operatorname{Adm}_{V}+\Lambda_{V}$. We can extend this statement to

Lemma 4.1.3. For $\lambda \in A d m_{V}+\Lambda_{V}$ holds $C_{\lambda} \succcurlyeq A$, in particular $L_{C_{\lambda}}^{2} \subseteq L_{A}^{2}$.

There is even a function $\delta: A d m_{V}+\Lambda_{V} \rightarrow \mathbb{R}^{+}$with $C_{\lambda}-\delta(\lambda) A \succcurlyeq 0$ such that $\lambda \mapsto \frac{1}{\delta(\lambda)}$ is locally bounded.

Proof. Let $k \in \operatorname{Adm}_{V}$ and $\nu \in \Lambda_{V}$ with $\lambda=k+\nu$. With $C_{k} \succcurlyeq 0$ and $-\boldsymbol{\operatorname { R e }}(\nu V J A) \succcurlyeq A$ we can estimate

$$
C_{\lambda}=C_{k}-\boldsymbol{\operatorname { R e }}(\nu V J A) \succcurlyeq-\boldsymbol{R e}(\nu V J A) \succcurlyeq A .
$$

$A \nprec 0$ and (4.3) ensure that for $\lambda \in \operatorname{Adm}_{V}+\Lambda_{V}$ we can choose some $\delta(\lambda)>0$ such that $C_{\lambda}-\delta(\lambda) A \succcurlyeq 0$ and $C_{\lambda}-2 \delta(\lambda) A \nsucc 0$.

Let $\delta(\lambda)$ be chosen like this for every $\lambda \in \operatorname{Adm}_{V}+\Lambda_{V}$.

Corollary 4.1.2 $(v)$ ensures that there exists some $\varepsilon>0$ such that

$$
A \pm \varepsilon \mathbf{R e} V J A \succcurlyeq 0 \quad \text { and } \quad A \pm \varepsilon \operatorname{Re} i V J A \succcurlyeq 0 .
$$

Let $\mu \in \operatorname{Adm}_{V}+\Lambda_{V}$.

For every $\lambda \in \operatorname{Adm}_{V}+\Lambda_{V}$ with $|\lambda-\mu| \leq \frac{1}{4} \delta(\mu) \varepsilon$ and a.e. $x \in[a, b)$ we can calculate:

$$
\begin{aligned}
C_{\lambda}(x) & =C_{\mu}(x)-\boldsymbol{\operatorname { R e }}((\lambda-\mu) V J A(x)) \\
& =C_{\mu}(x)-\boldsymbol{\operatorname { R e }}(\lambda-\mu) \operatorname{Re}(V J A(x))-\mathbf{I m}(\lambda-\mu) \operatorname{Re}(i V J A(x)) \\
& \geq \delta(\mu) A(x)-|\boldsymbol{R e}(\lambda-\mu)|_{\varepsilon}^{1} A(x)-|\mathbf{I m}(\lambda-\mu)|_{\varepsilon}^{1} A(x) \geq \frac{1}{2} \delta(\mu) A(x) .
\end{aligned}
$$


Thus $\delta(\lambda) \geq \frac{1}{4} \delta(\mu)$, which yields the assertion.

From now on we use the already mentioned shorter notation $W_{V}=\operatorname{Adm}_{V}+\Lambda_{V}$.

Lemma 4.1.4. Let $\lambda, \mu \in W_{V}$. Then $C_{\lambda} \asymp C_{\mu}$. In particular, $L_{C_{\lambda}}^{2}$ and $L_{C_{\mu}}^{2}$ contain the same functions and have equivalent norms.

Proof. Using Corollary 4.1.2 $(v)$ and Lemma 4.1.3, we can estimate

$$
C_{\lambda}=C_{\mu}+\operatorname{Re}((\mu-\lambda) V J A) \preccurlyeq C_{\mu}+A \preccurlyeq C_{\mu}+C_{\mu} \preccurlyeq C_{\mu} .
$$

By symmetry, the statement follows.

Lemma 4.1.5. Let $\lambda_{1}, \lambda_{2}, \lambda_{3}, \lambda_{4} \in W_{V}$. Then

$$
C_{\lambda_{1}} \asymp A \Longleftrightarrow \widehat{C_{\lambda_{2}}} \asymp A \Longrightarrow C_{\lambda_{3}} \asymp \widehat{C_{\lambda_{4}}} \text {. }
$$

Proof. With Lemma 4.1.4, the corresponding statement for the adjoint problem, and the transitivity of $\asymp$, it suffices to show the assertion for $\lambda_{1}=\lambda_{2}=\lambda_{3}=\lambda_{4}=: \lambda$.

Let $\widehat{C_{\lambda}} \asymp A$. Using (3.26), Corollary 4.1.2 (iv) and (3.10), we obtain

$$
C_{\lambda}=V J \widehat{C_{\lambda}} J^{*} V \asymp V J A J^{*} V \asymp A \asymp \widehat{C_{\lambda}} .
$$

With the symmetric argument, the whole assertion follows.

Finally we have

Lemma 4.1.6. If $B$ is self-adjoint and $V= \pm i J$, then $A \asymp C_{\lambda}$ for $\lambda \in W_{V}$.

Proof. For $\lambda \in W_{V}$ we know $C_{\lambda} \succcurlyeq A$. The statement follows by the calculation

$$
C_{\lambda}=-\operatorname{Re} V J(\lambda A+B)= \pm \boldsymbol{R e} i(\lambda A+B)= \pm \mathbf{R e}(i \lambda) A \preccurlyeq A .
$$

So in all our self-adjoint examples with the canonical choice of $V$ we have $A \asymp C_{\lambda}$. In particular in Example $\mathbf{1 . 3}$ on page 28 with $J=J_{2}$ we obtain

$$
A_{2} \asymp C_{\lambda, V_{c, 2}}=C_{\lambda, V_{c, 2}, J_{2}}
$$

for $\lambda$ in the lower halfplane. But also for the original choice, $J=J_{1}$ (see (2.34)), as in equation (2.43), we can calculate

$C_{\lambda, V_{c, 2}}=C_{\lambda, V_{c, 2}, J_{1}}=-\boldsymbol{R e}\left(V_{c, 2} J_{1}\left(k A_{1}+B_{1}\right)\right)=-\boldsymbol{R e}\left(V_{c, 2} J_{2}\left(k A_{2}+B_{2}\right)\right)=C_{\lambda, V_{c, 2}, J_{2}} \asymp A_{2}=A_{1}$. 
Again, the actual choice of $J$ has no influence on this matter.

In Example 3 on page 30 we have the even order problem with $m=1$. We know $A_{1}=$ $\operatorname{diag}(w, 0)$. Furthermore any suitable matrix is of the shape $V=\left(\begin{array}{cc}v_{0} & \bar{v} \\ v & 0\end{array}\right)$. For such a suitable $V$ with $\operatorname{Re} v>0,(2.48)$ shows $A \nprec C_{\lambda}$.

\subsection{The $M$-function}

In this section we want to construct an analytic $M$-function with $M(\lambda) \in D_{\lambda}(b)$ (with $D_{\lambda}(b)$ defined in Theorem 2.2.7) for $\lambda \in W_{V}$.

For $\lambda \in W_{V}$ and $l \in D_{\lambda}(b)$, we define

$$
\begin{aligned}
\Psi_{\lambda, l} & :=\Theta_{\lambda}+\Phi_{\lambda} l, \\
\zeta_{\lambda, l} & :=\eta_{\lambda}+\chi_{\lambda} l^{*} .
\end{aligned}
$$

By definition, $l \in D_{\lambda}(c)$ for every $c \in[a, b)$, and so (2.16) yields

$$
\int_{a}^{c} \Psi_{\lambda, l}^{*} C_{\lambda} \Psi_{\lambda, l} d x \leq \mathcal{A}_{\lambda}(l)
$$

Thus, for every $\xi \in \mathbb{C}^{n^{-}}$, we obtain

$$
\Psi_{\lambda, l} \xi \in L_{C_{\lambda}}^{2}
$$

and furthermore, using Lemma 4.1.3,

$$
\Psi_{\lambda, l} \xi \in L_{A}^{2}
$$

Since $\widehat{D_{\lambda}}(b)=D_{\lambda}(b)^{*}$ by Lemma 3.3.1, with the same argument we obtain, for $\xi \in \mathbb{C}^{n^{+}}$,

$$
\zeta_{\lambda, l} \xi \in L_{\widehat{C_{\lambda}}}^{2} \subseteq L_{A}^{2}
$$

For arbitrary $n_{f}, n_{g} \in \mathbb{N}_{0}$ and functions $f:[a, b) \rightarrow \mathbb{C}^{n, n_{f}}, g:[a, b) \rightarrow \mathbb{C}^{n, n_{g}}$, we use the notations

$$
[f, g](x):=g^{*}(x) J f(x) \quad(x \in[a, b))
$$


and

$$
[f, g](b):=\lim _{x \rightarrow b}[f, g](x) \quad \text { if the limit exists. }
$$

Using equation (3.22), we obtain

\section{Corollary 4.2.1.}

$$
\left(\begin{array}{cc}
0 & -I_{n^{+}} \\
I_{n^{-}} & 0
\end{array}\right)=-J_{0}^{*}=Z_{\lambda}^{*} J Y_{\lambda}=\left[Y_{\lambda}, Z_{\lambda}\right]=\left(\begin{array}{cc}
{\left[\Theta_{\lambda}, \eta_{\lambda}\right]} & {\left[\Phi_{\lambda}, \eta_{\lambda}\right]} \\
{\left[\Theta_{\lambda}, \chi_{\lambda}\right]} & {\left[\Phi_{\lambda}, \chi_{\lambda}\right]}
\end{array}\right)
$$

We need one more technical

Lemma 4.2.2. ${ }^{* 2}$ Let $\lambda, \mu \in W_{V}$ and $l \in D_{\mu}(b)$. For $c \in\left[c_{0}(\lambda), b\right)$, the regular boundary value problem

$$
\begin{aligned}
& J y^{\prime}=(\lambda A+B) y \quad \text { on }(a, c) \\
& {\left[y, \chi_{\mu}\right](a)=0,} \\
& {\left[y, \zeta_{\mu, l}\right](c)=0}
\end{aligned}
$$

has only the trivial solution.

Proof. Let $y$ be a solution of (4.12). Since $\left(\Theta_{\lambda} \mid \Phi_{\lambda}\right)$ is a fundamental matrix for the differential equation, there exists $\xi_{1} \in \mathbb{C}^{n^{-}}, \xi_{2} \in \mathbb{C}^{n^{+}}$such that $y=\Theta_{\lambda} \xi_{1}+\Phi_{\lambda} \xi_{2}$.

Since $\chi_{\lambda}(a)=\chi_{\mu}(a),(4.11)$ and the boundary condition at $a$ imply

$$
0=\left[y, \chi_{\lambda}\right](a)=\left[\Theta_{\lambda}, \chi_{\lambda}\right](a) \xi_{1}+\left[\Phi_{\lambda}, \chi_{\lambda}\right](a) \xi_{2}=\xi_{1} .
$$

Using (4.11) again (with $\lambda$ replaced by $\mu$ ), we can conclude

$$
\left[\Psi_{\mu, l}, \zeta_{\mu, l}\right](c)=\left(I_{n^{+}}, l\right)\left[Y_{\mu}, Z_{\mu}\right](c)\left(\begin{array}{c}
I_{n^{-}} \\
l
\end{array}\right)=\left(I_{n^{+}}, l\right)\left(\begin{array}{cc}
0 & -I_{n^{+}} \\
I_{n^{-}} & 0
\end{array}\right)\left(\begin{array}{c}
I_{n^{-}} \\
l
\end{array}\right)=0 .
$$

Since $\Psi_{\mu, l}$ and $\zeta_{\mu, l}$ have full ranks $n^{-}$and $n^{+}$, respectively, the boundary condition at $c$ implies $y=\Psi_{\mu, l} \xi$ for some $\xi \in \mathbb{C}^{n^{-}}$.

Thus, since $l \in D_{\mu}(b) \subseteq D_{\mu}(c)$, equation (2.11) yields

$$
y^{*}(c) V y(c) \leq 0
$$

If $\xi_{2}$ were not zero, Lemma 2.2.4 (iii) would imply

$$
y^{*}(c) V y(c)=\xi_{2}^{*} \Phi_{\lambda}^{*}(c) V \Phi_{\lambda}(c) \xi_{2}=2 \xi_{2}^{*} P(c) \xi_{2}>0,
$$

a contradiction. Hence $\xi_{2}=0$ and thus $y=0$. 
Now we can state the existence of an $M$-function in

Theorem 4.2.3. ${ }^{* 2}$ For any $\mu \in W_{V}$ and $M_{0} \in D_{\mu}(b)$, there exists a function

$$
M: W_{V} \longrightarrow \mathbb{C}^{n^{+}, n^{-}}
$$

such that

(i) $M(\mu)=M_{0}$

and, for every $\lambda \in W_{V}$,

(ii) $M(\lambda) \in D_{\lambda}(b)$,

$$
\begin{aligned}
M(\lambda)-M_{0} & =(\lambda-\mu) \int_{a}^{b} \zeta_{\mu, M_{0}}^{*} A \Psi_{\lambda, M(\lambda)} d x \\
& =(\lambda-\mu) \int_{a}^{b} \zeta_{\lambda, M(\lambda)}^{*} A \Psi_{\mu, M_{0}} d x .
\end{aligned}
$$

Proof. We first show that, for $\lambda \in W_{V}$ and $c \in\left[c_{0}(\lambda), b\right)$, the matrix $\left[\Phi_{\lambda}, \zeta_{\mu, M_{0}}\right](c)=$ $\zeta_{\mu, M_{0}}(c)^{*} J \Phi_{\lambda}(c)$ is invertible:

Let $\xi \in \mathbb{C}^{n^{+}}$be in its null-space. The function $\Phi_{\lambda} \xi$ solves the boundary value problem (4.12), since $\Phi_{\lambda}$ solves the differential equation; (4.11) yields the boundary condition at $a$; note that $\chi_{\lambda}(a)=\chi_{\mu}(a)$. Thus $\Phi_{\lambda} \xi=0$ by Lemma 4.2 .2 , which yields $\xi=0$ because $\Phi_{\lambda}$ has rank $n^{+}$.

Therefore we can define, on $\left[c_{0}(\lambda), b\right)$,

$$
l_{\lambda, c}:=-\left(\left[\Phi_{\lambda}, \zeta_{\mu, M_{0}}\right](c)\right)^{-1}\left[\Theta_{\lambda}, \zeta_{\mu, M_{0}}\right](c) .
$$

By this definition we obtain, for $c \in\left[c_{0}(\lambda), b\right)$,

$$
\left[\Psi_{\lambda, l_{\lambda, c}}, \zeta_{\mu, M_{0}}\right](c)=\left[\Theta_{\lambda}, \zeta_{\mu, M_{0}}\right](c)+\left[\Phi_{\lambda}, \zeta_{\mu, M_{0}}\right](c) l_{\lambda, c}=0
$$

whereas (4.13) gives

$$
\left[\Psi_{\mu, M_{0}}, \zeta_{\mu, M_{0}}\right](c)=0
$$

(4.15) and (4.16) yield that the ranges of both $\Psi_{\mu, M_{0}}(c)$ and $\Psi_{\lambda, l_{\lambda, c}}(c)$ are in the null-space of $\zeta_{\mu, M_{0}}^{*}(c) J$, which has dimension $n^{-}$because $\zeta_{\mu, M_{0}}$ has rank $n^{+}$. Furthermore $\Psi_{\mu, M_{0}}(c)$ and $\Psi_{\lambda, l_{\lambda, c}}(c)$ both have rank $n^{-}$, hence their ranges coincide. Thus we obtain

$$
\forall \lambda \in W_{V}, c \in\left[c_{0}(\lambda), b\right): \operatorname{range}\left(\Psi_{\lambda, l_{\lambda, c}}(c)\right)=\operatorname{range}\left(\Psi_{\mu, M_{0}}(c)\right) \text {. }
$$


Consequently, there exists $\Xi_{\lambda} \in \mathbb{C}^{n^{-}, n^{-}}$such that

$$
\Psi_{\lambda, l_{\lambda, c}}(c)=\Psi_{\mu, M_{0}}(c) \Xi_{\lambda}
$$

Since $M_{0} \in D_{\mu}(c)$, Definition (2.11) yields

$$
\Psi_{\lambda, l_{\lambda, c}}^{*}(c) V \Psi_{\lambda, l_{\lambda, c}}(c)=\Xi_{\lambda}^{*} \Psi_{\mu, M_{0}}^{*}(c) V \Psi_{\mu, M_{0}}(c) \Xi_{\lambda} \leq 0
$$

so, again by (2.11),

$$
l_{\lambda, c} \in D_{\lambda}(c)
$$

With corresponding arguments for the adjoint problem (including an adjoint version of Lemma 4.2.2) we conclude that for all $\lambda \in W_{V}$ the ranges of $\zeta_{\lambda, l_{\lambda, c}}(c)$ and of $\zeta_{\mu, M_{0}}(c)$ coincide. Therewith, equations (4.17) and (4.16) imply

$$
\forall \nu, \lambda \in W_{V}, c \in\left[c_{0}(\lambda), b\right) \cap\left[c_{0}(\nu), b\right): \quad\left[\Psi_{\lambda, l_{\lambda, c}}, \zeta_{\nu, l_{\nu, c}}\right](c)=0 .
$$

For $\nu, \lambda \in W_{V}$ and $c \in\left[c_{0}(\lambda), b\right) \cap\left[c_{0}(\nu), b\right)$, we obtain by (2.1), (3.15), and $J^{*}=-J$ that

$$
\left[\Psi_{\lambda, l_{\lambda, c}}, \zeta_{\nu, l_{\nu, c}}\right]^{\prime}=\left(\zeta_{\nu, l_{\nu, c}}^{*} J \Psi_{\lambda, l_{\lambda, c}}\right)^{\prime}=(\lambda-\nu) \zeta_{\nu, l_{\nu, c}}^{*} A \Psi_{\lambda, l_{\lambda, c}}
$$

Integrating from $a$ to $c$ and use of (4.19), (4.11) and $Z_{\nu}(a)=\widehat{E}=Z_{\lambda}(a)$ yields

$$
\begin{aligned}
(\lambda-\nu) \int_{a}^{c} \zeta_{\nu, l_{\nu, c}}^{*} A \Psi_{\lambda, l_{\lambda, c}} d x & =-\left[\Psi_{\lambda, l_{\lambda, c}}, \zeta_{\nu, l_{\nu, c}}\right](a) \\
& =-\left(I_{n^{+}}, l_{\nu, c}\right)\left[Y_{\lambda}, Z_{\lambda}\right](a)\left(\begin{array}{l}
I_{n^{-}} \\
l_{\lambda, c}
\end{array}\right) \\
& =\left(I_{n^{+}}, l_{\nu, c}\right)\left(\begin{array}{cc}
0 & I_{n^{+}} \\
-I_{n^{-}} & 0
\end{array}\right)\left(\begin{array}{l}
I_{n^{-}} \\
l_{\lambda, c}
\end{array}\right)=l_{\lambda, c}-l_{\nu, c} .
\end{aligned}
$$

The same calculation with (4.15) and $\left(\mu, M_{0}\right)$ instead of $(4.19)$ and $\left(\nu, l_{\nu, c}\right)$ yields

$$
l_{\lambda, c}-M_{0}=(\lambda-\mu) \int_{a}^{c} \zeta_{\mu, M_{0}}^{*} A \Psi_{\lambda, l_{\lambda, c}} d x .
$$

With $\lambda=\mu$, this implies $l_{\mu, c}=M_{0}$.

So (4.21), with changed roles of $\nu$ and $\lambda$, and then $\nu$ put equal to $\mu$, reads

$$
l_{\lambda, c}-M_{0}=(\lambda-\mu) \int_{a}^{c} \zeta_{\lambda, l_{\lambda, c}}^{*} A \Psi_{\mu, M_{0}} d x
$$

We fix some $\lambda \in W_{V}$ for the rest of the proof. 
Using Lemma 4.1.3 and (2.12), we conclude that there exists a constant $k$ such that, for $c \in\left[c_{0}(\lambda), b\right)$,

$$
0 \leq \int_{a}^{c} \Psi_{\lambda, l_{\lambda, c}}^{*} A \Psi_{\lambda, l_{\lambda, c}} d x \leq k \int_{a}^{c} \Psi_{\lambda, l_{\lambda, c}}^{*} C_{\lambda} \Psi_{\lambda, l_{\lambda, c}} d x=\left.\frac{1}{2} k \Psi_{\lambda, l_{\lambda, c}}^{*} V \Psi_{\lambda, l_{\lambda, c}}\right|_{a} ^{c} .
$$

The boundary term at $c$ is non-positive by (2.11) and (4.18). The term at $a$ is bounded with respect to $c \in\left[c_{0}(\lambda), b\right)$ by (4.5), since $l_{\lambda, c} \in D_{\lambda}(c) \subseteq D_{\lambda}\left(c_{0}(\lambda)\right)$ and the latter set is bounded by Theorem 2.2.7.

If we set $\tilde{\Psi}_{c}:=1_{[a, c]} \Psi_{\lambda, l_{\lambda, c}}$ the estimation above yields boundedness, and thus weak convergence of $\tilde{\Psi}_{c}$ in $L_{A}^{2}$ to some $F \in L_{A}^{2}$ at least along a sequence $\left(c_{m}\right)$ tending to $b$.

Since $\left(l_{\lambda, c_{m}}\right)$ is bounded we can assume w.l.o.g. that

$$
l_{\lambda, c_{m}} \rightarrow M(\lambda) \quad \text { as } \quad m \rightarrow \infty
$$

for some $M(\lambda) \in \mathbb{C}^{n^{+}, n^{-}}$.

Using Corollary 2.2.3, Theorem 2.2.7 and (4.18), we obtain

$$
M(\lambda) \in D_{\lambda}(b) .
$$

Thus, by (4.7) and (4.8), we know that all columns of $\Psi_{\lambda, M(\lambda)}$ and $\zeta_{\lambda, M(\lambda)}$ are in $L_{A}^{2}$.

By definition of $\tilde{\Psi}_{c},(4.25)$ yields locally uniform convergence of $\tilde{\Psi}_{c}$ to $\Psi_{\lambda, M(\lambda)}$. The compactly supported test-functions are dense in $L_{A}^{2}$, thus $F=\Psi_{\lambda, M(\lambda)}$ in $L_{A}^{2}$.

Because $\zeta_{\mu, M_{0}} \in L_{A}^{2}$, the weak convergence $\tilde{\Psi}_{c_{m}} \rightarrow \Psi_{\lambda, M(\lambda)}$ shows in particular that

$$
\int_{a}^{b} \zeta_{\mu, M_{0}}^{*} A \tilde{\Psi}_{c_{m}} d x \rightarrow \int_{a}^{b} \zeta_{\mu, M_{0}}^{*} A \Psi_{\lambda, M(\lambda)} d x \quad \text { as } \quad m \rightarrow \infty
$$

Using (4.22) and (4.25), we obtain

$$
\begin{aligned}
M(\lambda)-M_{0} & =\lim _{m \rightarrow \infty} l_{\lambda, c_{m}}-M_{0} \\
& =\lim _{m \rightarrow \infty}(\lambda-\mu) \int_{a}^{c_{m}} \zeta_{\mu, M_{0}}^{*} A \Psi_{\lambda, l_{\lambda, c_{m}}} d x \\
& =\lim _{m \rightarrow \infty}(\lambda-\mu) \int_{a}^{b} \zeta_{\mu, M_{0}}^{*} A \tilde{\Psi}_{c_{m}} d x=(\lambda-\mu) \int_{a}^{b} \zeta_{\mu, M_{0}}^{*} A \Psi_{\lambda, M(\lambda)} d x .
\end{aligned}
$$

The second equation of statement (iii) can be shown analogously, with exchanged roles of $\zeta$ and $\Psi$ and use of (4.23). W.l.o.g. we can assume that the limit $M(\lambda)$ is the same as before. 


\subsection{The resolvent operator}

In this section we define (at least on $W_{V}$ ) the $\lambda$-dependent functions $R_{\lambda}$ via a kernel $G_{\lambda}$. It will turn out that $R_{\lambda}$ is the resolvent of the differential operator we are looking for.

For the following we fix some $\mu \in W_{V}, M_{0} \in D_{\mu}(b)$, and a corresponding $M$-function given by Theorem 4.2.3.

For $\lambda \in W_{V}$, let

$$
\begin{aligned}
\Psi_{\lambda}:=\Psi_{\lambda, M(\lambda)} & =\Theta_{\lambda}+\Phi_{\lambda} M(\lambda), \\
\zeta_{\lambda}:=\zeta_{\lambda, M(\lambda)} & =\eta_{\lambda}+\chi_{\lambda} M(\lambda)^{*} .
\end{aligned}
$$

Lemma 4.3.1. ${ }^{*_{2}}$ For $\lambda \in W_{V}$,

$$
\begin{aligned}
& {\left[\Psi_{\lambda}, \zeta_{\mu}\right](b)=0,} \\
& {\left[\Psi_{\mu}, \zeta_{\lambda}\right](b)=0 .}
\end{aligned}
$$

Proof. Using $Z_{\lambda}(a)=\widehat{E}=Z_{\mu}(a)$, we obtain from (4.11), by multiplication with $\left(\begin{array}{c}I_{n^{-}} \\ M(\lambda)\end{array}\right)$ from the right and with $\left(I_{n^{+}} \mid M(\mu)\right)$ from the left,

$$
\left[\Psi_{\lambda}, \zeta_{\mu}\right](a)=\left(I_{n^{+}} \mid M(\mu)\right)\left(\begin{array}{cc}
0 & -I_{n^{+}} \\
I_{n^{-}} & 0
\end{array}\right)\left(\begin{array}{c}
I_{n^{-}} \\
M(\lambda)
\end{array}\right)=M(\mu)-M(\lambda)
$$

Since $\Psi_{\lambda}$ and $\zeta_{\mu}$ solve (2.1) and (3.15), respectively, and $J^{*}=-J$,

$$
\left[\Psi_{\lambda}, \zeta_{\mu}\right]^{\prime}=\left(\zeta_{\mu}^{*} J \Psi_{\lambda}\right)^{\prime}=(\lambda-\mu) \zeta_{\mu}^{*} A \Psi_{\lambda} .
$$

Integration provides, for all $c \in[a, b)$,

$$
\begin{aligned}
{\left[\Psi_{\lambda}, \zeta_{\mu}\right](c) } & =\left[\Psi_{\lambda}, \zeta_{\mu}\right](a)+(\lambda-\mu) \int_{a}^{c} \zeta_{\mu}^{*} A \Psi_{\lambda} d x \\
& =M(\mu)-M(\lambda)+(\lambda-\mu) \int_{a}^{c} \zeta_{\mu}^{*} A \Psi_{\lambda} d x
\end{aligned}
$$

The right-hand side converges to zero by Theorem 4.2 .3 (iii), as $c \rightarrow b$, which proves (4.30).

Equation (4.31) can be proven correspondingly, using the second equation of Theorem $4.2 .3($ iii) . 
We further define

$$
\begin{aligned}
& G_{\lambda}(x, y):= \begin{cases}\Phi_{\lambda}(x) \zeta_{\lambda}^{*}(y) & \text { if } a \leq x \leq y<b, \\
\Psi_{\lambda}(x) \chi_{\lambda}^{*}(y) & \text { if } a \leq y<x<b,\end{cases} \\
& \widehat{G}_{\lambda}(x, y):= \begin{cases}\chi_{\lambda}(x) \Psi_{\lambda}^{*}(y) & \text { if } a \leq x<y<b, \\
\zeta_{\lambda}(x) \Phi_{\lambda}^{*}(y) & \text { if } a \leq y \leq x<b\end{cases} \\
& =G_{\lambda}^{*}(y, x),
\end{aligned}
$$

and for $f \in L_{A}^{2}$

$$
\begin{aligned}
& R_{\lambda} f(x):=\int_{a}^{b} G_{\lambda}(x, y) A(y) f(y) d y, \\
& \widehat{R}_{\lambda} f(x):=\int_{a}^{b} \widehat{G}_{\lambda}(x, y) A(y) f(y) d y .
\end{aligned}
$$

By definition,

$$
R_{\lambda} f(x)=Y_{\lambda}(x) \int_{a}^{b}\left(\begin{array}{cc}
0 & 1_{(a, x)} I_{n^{-}} \\
1_{(x, b)} I_{n^{+}} & M(\lambda)
\end{array}\right)(y) Z_{\lambda}^{*}(y) A(y) f(y) d y .
$$

With (3.23), this yields

Corollary 4.3.2. ${ }^{*_{1}} \quad R_{\lambda} f \in A C_{l o c}[a, b)$ with

$$
J\left(R_{\lambda} f\right)^{\prime}=(\lambda A+B)\left(R_{\lambda} f\right)-J Y_{\lambda} J_{0} Z_{\lambda}^{*} A f=(\lambda A+B)\left(R_{\lambda} f\right)+A f .
$$

Using (4.36), (4.11), and (4.8), we obtain*1

$$
\begin{aligned}
{\left[R_{\lambda} f, \chi_{\lambda}\right](a) } & =0 \\
{\left[R_{\lambda} f, \zeta_{\lambda}\right](b) } & =\lim _{x \rightarrow b}\left(I_{n^{+}} \mid M(\lambda)\right)\left[Y_{\lambda}, Z_{\lambda}\right](x) \int_{a}^{b}\left(\begin{array}{cc}
0 & 1_{(a, x)} I_{n^{-}} \\
1_{(x, b)} I_{n^{+}} & M(\lambda)
\end{array}\right)(y) Z_{\lambda}^{*}(y) A(y) f(y) d y \\
& =\lim _{x \rightarrow b}\left(I_{n^{+}} \mid M(\lambda)\right)\left(\begin{array}{cc}
0 & -I_{n^{+}} \\
I_{n^{-}} & 0
\end{array}\right) \int_{a}^{b}\left(\begin{array}{cc}
0 & 1_{(a, x)} I_{n^{-}} \\
1_{(x, b)} I_{n^{+}} & M(\lambda)
\end{array}\right)(y) Z_{\lambda}^{*}(y) A(y) f(y) d y \\
& =\lim _{x \rightarrow b} \int_{a}^{b}\left(-1_{(x, b)} I_{n^{+}} \mid 1_{(a, x)} M(\lambda)-M(\lambda)\right)(y) Z_{\lambda}^{*}(y) A(y) f(y) d y \\
& =-\lim _{x \rightarrow b} \int_{x}^{b} \zeta_{\lambda}^{*}(y) A(y) f(y) d y=0 .
\end{aligned}
$$


Analogous calculations for the adjoint problem yield ${ }^{*_{1}}$

$$
\begin{aligned}
& J\left(\widehat{R}_{\lambda} f\right)^{\prime}=\left(\bar{\lambda} A+B^{*}\right)\left(\widehat{R}_{\lambda} f\right)+A f, \\
& {\left[\widehat{R}_{\lambda} f, \Phi_{\lambda}\right](a)=0,} \\
& {\left[\widehat{R}_{\lambda} f, \Psi_{\lambda}\right](b)=0 .}
\end{aligned}
$$

Theorem 4.3.3.*3 For each $\lambda \in W_{V}, R_{\lambda}$ is a bounded operator $L_{A}^{2} \rightarrow L_{A}^{2}, L_{C_{\lambda}}^{2} \rightarrow L_{C_{\lambda}}^{2}$, and $L_{A}^{2} \rightarrow L_{C_{\lambda}}^{2}$.

Furthermore, $\lambda \mapsto\left\|R_{\lambda}\right\|_{A}$ and $\lambda \mapsto\left\|R_{\lambda}\right\|_{C_{\lambda}}$ are locally bounded.

Proof. By Corollary 4.1.2 (iv) there exists $\varepsilon>0$ with $A-\varepsilon V J A J^{*} V \succcurlyeq 0$.

Lemma 4.1 .3 yields a function $\delta: W_{V} \rightarrow \mathbb{R}^{+}$with $C_{\lambda}-\delta(\lambda) A \succcurlyeq 0$ on $W_{V}$, such that $\frac{1}{\delta}$ is locally bounded on $W_{V}$. Thus,

$$
C_{\lambda}-\varepsilon \delta(\lambda) V J A J^{*} V \succcurlyeq 0 .
$$

We will show that $\left\|R_{\lambda}\right\|_{A},\left\|R_{\lambda}\right\|_{C_{\lambda}} \leq \frac{1}{\sqrt{\varepsilon \delta(\lambda)}}$, which proves the desired locally boundedness.

Let $\lambda \in W_{V}$ and let $f \in L_{A}^{2}$. For $c \in[a, b)$ we set $f_{c}:=1_{[a, c]} f$ and $\Upsilon:=R_{\lambda} f_{c}$. Using (4.37) and the chain rule, we obtain

$$
\begin{aligned}
2 \int_{a}^{c} \Upsilon^{*} C_{\lambda} \Upsilon d x & =\int_{a}^{c}-\left(R_{\lambda} f_{c}\right)^{*} 2 \mathbf{R e}(V J(\lambda A+B))\left(R_{\lambda} f_{c}\right) d x \\
& =-2 \mathbf{R e} \int_{a}^{c}\left(R_{\lambda} f_{c}\right)^{*} V J(\lambda A+B)\left(R_{\lambda} f_{c}\right) d x \\
& =-2 \mathbf{R e} \int_{a}^{c}\left(R_{\lambda} f_{c}\right)^{*} V J\left(J\left(R_{\lambda} f_{c}\right)^{\prime}-A f_{c}\right) d x \\
& =2 \mathbf{R e} \int_{a}^{c} \Upsilon^{*} V \Upsilon^{\prime}+\Upsilon^{*} V J A f_{c} d x \\
& =\int_{a}^{c} \Upsilon^{*} V \Upsilon^{\prime}+\left(\Upsilon^{*}\right)^{\prime} V \Upsilon+2 \mathbf{R e}\left(\Upsilon^{*} V J A f_{c}\right) d x \\
& =\left.\Upsilon^{*} V \Upsilon\right|_{a} ^{c}+2 \mathbf{R e} \int_{a}^{c} \Upsilon^{*} V J A f_{c} d x
\end{aligned}
$$

Moreover, since $\operatorname{supp}\left(f_{c}\right) \subseteq[a, c],(4.36)$ implies

$$
\Upsilon(c)=Y_{\lambda}(c)\left(\begin{array}{c}
I_{n^{-}} \\
M(\lambda)
\end{array}\right) F
$$

for $F:=\int_{a}^{c} \chi_{\lambda}^{*} A f_{c} d x$. Thus,

$$
\left(\Upsilon^{*} V \Upsilon\right)(c)=F^{*}\left(\Theta_{\lambda}+\Phi_{\lambda} M(\lambda)\right)^{*} V\left(\Theta_{\lambda}+\Phi_{\lambda} M(\lambda)\right) F \leq 0,
$$


by $(2.11)$, since $M(\lambda) \in D_{\lambda}(c)$.

Using $Y_{\lambda}(a)=E$ and (4.36) again, we obtain

$$
\Upsilon(a)=E\left(\begin{array}{cc}
0 & 0 \\
I_{n^{+}} & M(\lambda)
\end{array}\right) G
$$

for $G:=\int_{a}^{c} Z_{\lambda}^{*} A f_{c} d x$. Thus, by (2.18) and (2.9)

$$
\left(\Upsilon^{*} V \Upsilon\right)(a)=2 G^{*}\left(\begin{array}{c}
I_{n^{+}} \\
M(\lambda)^{*}
\end{array}\right) P_{\lambda}(a)\left(I_{n^{+}} \mid M(\lambda)\right) G \geq 0 .
$$

Using (4.41), (4.43), (4.45) and (4.40), together with the Cauchy-Schwarz inequality in $L_{A}^{2}(a, c)$, we can estimate

$$
\begin{aligned}
\int_{a}^{c} \Upsilon^{*} C_{\lambda} \Upsilon d x & \leq\left|\int_{a}^{c} \Upsilon^{*} V J A f_{c} d x\right| \\
& \leq\left(\int_{a}^{c} f_{c}^{*} A f_{c} d x\right)^{1 / 2}\left(\int_{a}^{c} \Upsilon^{*} V J A J^{*} V \Upsilon d x\right)^{1 / 2} \\
& \leq \frac{1}{2 \varepsilon \delta(\lambda)}\|f\|_{A}^{2}+\frac{1}{2} \varepsilon \delta(\lambda)\|\Upsilon\|_{V J A J^{*} V,[a, c]}^{2} \\
& \leq \frac{1}{2 \varepsilon \delta(\lambda)}\|f\|_{A}^{2}+\frac{1}{2}\|\Upsilon\|_{C_{\lambda},[a, c]}^{2} \\
& =\frac{1}{2 \varepsilon \delta(\lambda)}\|f\|_{A}^{2}+\frac{1}{2} \int_{a}^{c} \Upsilon^{*} C_{\lambda} \Upsilon d x
\end{aligned}
$$

Thus we have, for $d \in[a, b)$ and $c \in[d, b)$,

$$
\int_{a}^{d}\left(R_{\lambda} f_{c}\right)^{*} C_{\lambda} R_{\lambda} f_{c}=\int_{a}^{d} \Upsilon^{*} C_{\lambda} \Upsilon d x \leq \int_{a}^{c} \Upsilon^{*} C_{\lambda} \Upsilon d x \leq \frac{1}{\varepsilon \delta(\lambda)}\|f\|_{A}^{2} .
$$

If we define

$$
k(c):=\int_{c}^{b} \zeta_{\lambda}^{*}(y) A(y) f(y) d y
$$

then $k(c) \rightarrow 0$ for $c \rightarrow b$, since $\zeta_{\lambda}, f \in L_{A}^{2}$. By (4.34) and (4.35), we obtain $R_{\lambda}\left(f-f_{c}\right)=$ $\Phi_{\lambda} k(c)$ on $[a, d]$, for $d<c$.

Therefore, on $[a, d]$,

$$
\begin{aligned}
& \left(R_{\lambda} f\right)^{*} C_{\lambda} R_{\lambda} f-\left(R_{\lambda} f_{c}\right)^{*} C_{\lambda} R_{\lambda} f_{c} \\
& =\left(R_{\lambda}\left(f-f_{c}\right)\right)^{*} C_{\lambda} R_{\lambda}(f)+\left(R_{\lambda} f\right)^{*} C_{\lambda} R_{\lambda}\left(f-f_{c}\right)-\left(R_{\lambda}\left(f-f_{c}\right)\right)^{*} C_{\lambda} R_{\lambda}\left(f-f_{c}\right) \\
& =k^{*}(c) \Phi_{\lambda}^{*} C_{\lambda} R_{\lambda}(f)+\left(R_{\lambda} f\right)^{*} C_{\lambda} \Phi_{\lambda} k(c)-k^{*}(c) \Phi_{\lambda}^{*} C_{\lambda} \Phi_{\lambda} k(c) .
\end{aligned}
$$


The integral over $[a, d]$ of this term tends to zero as $c$ tends to $b$. Thus (4.47) yields

$$
\int_{a}^{d}\left(R_{\lambda} f\right)^{*} C_{\lambda} R_{\lambda} f d x \leq \frac{1}{\varepsilon \delta(\lambda)}\|f\|_{A}^{2}
$$

for arbitrary $d \in[a, b)$. This shows $R_{\lambda} f \in L_{C_{\lambda}}^{2}$, and

$$
\left\|R_{\lambda} f\right\|_{C_{\lambda}}^{2} \leq \frac{1}{\varepsilon \delta(\lambda)}\|f\|_{A}^{2}
$$

Thus $R_{\lambda}$ is even a bounded operator $L_{A}^{2} \rightarrow L_{C_{\lambda}}^{2}$. Using $C_{\lambda} \succcurlyeq A$, we see that $R_{\lambda}$ is a bounded operator $L_{A}^{2} \rightarrow L_{A}^{2}$ and $L_{C_{\lambda}}^{2} \rightarrow L_{C_{\lambda}}^{2}$. More precisely, we can estimate

$$
\left\|R_{\lambda} f\right\|_{A}^{2} \leq \frac{1}{\delta(\lambda)}\left\|R_{\lambda} f\right\|_{C_{\lambda}}^{2} \leq \frac{1}{\varepsilon \delta^{2}(\lambda)}\|f\|_{A}^{2}
$$

and, for $f \in L_{C_{\lambda}}^{2} \subseteq L_{A}^{2}$,

$$
\left\|R_{\lambda} f\right\|_{C_{\lambda}}^{2} \leq \frac{1}{\varepsilon \delta(\lambda)}\|f\|_{A}^{2} \leq \frac{1}{\varepsilon \delta^{2}(\lambda)}\|f\|_{C_{\lambda}}^{2}
$$

Hence the assertions follows.

Theorem 4.3.3 and $\widehat{G}_{\lambda}(x, y)=G_{\lambda}^{*}(y, x)$ imply

Corollary 4.3.4. ${ }^{* 1} \widehat{R}_{\lambda}$ is the $L_{A^{-}}^{2}$ adjoint of $R_{\lambda}$ and vice versa.

\subsection{Definition and properties of the differential operator}

To define an operator for the formal problems (2.1) and (3.15), respectively, we need one more

Assumption 2. The operators $R_{\mu}, \widehat{R}_{\mu}: L_{A}^{2} \rightarrow L_{A}^{2}$ are one-to-one.

Theorem 4.4.8 below shows that under this assumption, for every $\lambda \in W_{V}$, the operators $R_{\lambda}$ and $\widehat{R}_{\lambda}$ are one-to-one from $L_{A}^{2}$ into itself.

The following remark is slightly technical but sometimes well applicable. 
Remark 4.4.1. If the sets $N_{m}$, given by

$$
\begin{aligned}
N_{1} & :=\operatorname{ker}(A) \\
N_{m+1} & :=N_{m} \cap B^{-1}\left(J N_{m}+\text { range } A\right),
\end{aligned}
$$

are constant on $[a, b)$ for all $m \in\{1, \ldots n\}$, and $N_{n}=\{0\}$, then $R_{\mu}$ is one-to-one for any $\mu \in W_{V}$. The corresponding statement holds for $\widehat{R}_{\mu}$ with $B^{*}$ in place of $B$.

Proof. Let $f \in L_{A}^{2}$ with $R_{\mu} f=0$ in $L_{A}^{2}$.

We set $\Upsilon:=R_{\mu} f$. Thus $\Upsilon$ is continuous and $A \Upsilon=0$ a.e. on $[a, b)$. Since ker $A$ is constant, we obtain $A \Upsilon(c)=0$ for every $c \in[a, b)$.

We show $\Upsilon(c) \in N_{m}$ for every $c \in[a, b)$ and $m \in \mathbb{N}$ by induction. The case $m=1$ holds by definition. Now let the assertion hold for $m \in \mathbb{N}$, and let $c \in[a, b)$.

For $\Upsilon(c)$ to be in $N_{m+1}$, we need to show

$$
\Upsilon(c) \in B^{-1}\left(J N_{m}+\text { range } A\right) .
$$

Since $N_{m}$ is constant, $\Upsilon^{\prime}(c) \in N_{m}$.

Using (4.37), we obtain $J \Upsilon^{\prime}=(\mu A+B) \Upsilon+A f=B \Upsilon+A f$.

Thus $B \Upsilon(c)=J \Upsilon^{\prime}(c)-A f \in J N_{m}+$ range $A$, which yields (4.50).

So in particular $\Upsilon(c) \in N_{n}=\{0\}$, and hence $\Upsilon(c)=\Upsilon^{\prime}(c)=0$ for every $c \in[a, b)$. Thus, $A f=J \Upsilon^{\prime}-B \Upsilon=0$ on $[a, b)$.

Another sufficient condition for Assumption 2 is given by

Remark 4.4.2. If, for almost every $x \in[a, b)$, either $A(x)=0$ or $A(x)>0$, then Assumption 2 is fulfilled.

Proof. It suffices to show injectivity of $R_{\mu}: L_{A}^{2} \rightarrow L_{A}^{2}$, by symmetry.

We assume the existence of some $f \in L_{A}^{2}, A f \neq 0$ on a set of positive measure, with $R_{\mu} f=0$ in $L_{A}^{2}$, i.e. $A R_{\mu} f=0$ a.e..

Let $a \leq c_{0}<c_{1}<b$ such that $A f=0$ a.e. on $\left[a, c_{0}\right]$ and

$$
\int_{c_{0}}^{c} A f d x \neq 0 \text { for all } c \in\left(c_{0}, c_{1}\right)
$$


We set $\mathcal{D}:=\left\{c \in\left(c_{0}, c_{1}\right): A(c)>0\right\}$. For a.e. $c \in \mathcal{D}$ we have $A(c) R_{\mu} f(c)=0$ and thus, by $(4.36)$,

$$
0=R_{\mu} f(c)=Y_{\mu}(c) \int_{a}^{b}\left(\begin{array}{cc}
0 & 1_{(a, c)} I_{n^{-}} \\
1_{(c, b)} I_{n^{+}} & M(\mu)
\end{array}\right)(x) Z_{\mu}^{*}(x) A(x) f(x) d x .
$$

Since $Y_{\mu}(c)$ is regular we even obtain

$$
0=\int_{a}^{b}\left(\begin{array}{cc}
0 & 1_{(a, c)} I_{n^{-}} \\
1_{(c, b)} I_{n^{+}} & M(\mu)
\end{array}\right)(x) Z_{\mu}^{*}(x) A(x) f(x) d x .
$$

Thus, for a.e. $c, \tilde{c} \in \mathcal{D}$,

$$
\begin{aligned}
0 & =\int_{a}^{b}\left(\begin{array}{cc}
0 & 1_{(a, \tilde{c})} I_{n^{-}} \\
1_{(\tilde{c}, b)} I_{n^{+}} & M(\mu)
\end{array}\right)(x) Z_{\mu}^{*}(x) A(x) f(x) d x-\int_{a}^{b}\left(\begin{array}{cc}
0 & 1_{(a, c)} I_{n^{-}} \\
1_{(c, b)} I_{n^{+}} & M(\mu)
\end{array}\right)(x) Z_{\mu}^{*}(x) A(x) f(x) d x \\
& =\int_{c}^{\tilde{c}}\left(\begin{array}{cc}
0 & I_{n^{-}} \\
-I_{n^{+}} & 0
\end{array}\right) Z_{\mu}^{*}(x) A(x) f(x) d x \\
& =\left(\begin{array}{cc}
0 & I_{n^{-}} \\
-I_{n^{+}} & 0
\end{array}\right) \int_{c}^{\tilde{c}} Z_{\mu}^{*}(x) A(x) f(x) d x
\end{aligned}
$$

and hence $0=\int_{c}^{\tilde{c}} Z_{\mu}^{*}(x) A(x) f(x) d x$. By (4.51) and the assumption on $A$ of this Remark, we can chose $c>c_{0}$ arbitrarily close to $c_{0}$, whence we obtain

$$
0=\int_{c_{0}}^{\tilde{c}} Z_{\mu}^{*}(x) A(x) f(x) d x
$$

for a.e. $\tilde{c} \in \mathcal{D}$ and therewith for all $\tilde{c} \in\left(c_{0}, c_{1}\right)$. (Otherwise, by continuity of the integral term with respect to $\tilde{c}$, there is an interval $\left(\tilde{c}_{0}, \tilde{c}_{1}\right) \subseteq\left(c_{0}, c_{1}\right)$ such that the integral term is zero for $\tilde{c}=\tilde{c}_{0}$, but is not zero for all $\tilde{c} \in\left(\tilde{c}_{0}, \tilde{c}_{1}\right)$. But the integrand is zero a.e. in $\left(\tilde{c}_{0}, \tilde{c}_{1}\right)$, because for $x \in\left(c_{0}, c_{1}\right) \backslash \mathcal{D}$ holds $A(x)=0$.)

This implies, for a.e. $x \in\left(c_{0}, c_{1}\right), Z_{\mu}^{*}(x) A(x) f(x)=0$, and thus $A(x) f(x)=0$ by the regularity of $Z_{\mu}$. This causes a contradiction to (4.51).

We are going to define the differential operator. Since $L_{A}^{2}$ consists of equivalent classes, we have to take care of well-definedness. For this purpose, we will use

Lemma 4.4.3.*2 Let $y_{1}, y_{2}:[a, b) \rightarrow \mathbb{C}^{n}$, with

(i) $y_{1}, y_{2} \in L_{A}^{2}, y_{1}=y_{2}$ in $L_{A}^{2}$,

(ii) $y_{1}, y_{2} \in A C_{l o c}[a, b)$,

(iii) $\left[y_{j}, \chi_{\mu}\right](a)=\left[y_{j}, \zeta_{\mu}\right](b)=0$ for $j \in\{1,2\}$. 
Then, for $f_{1}, f_{2} \in L_{A}^{2}$ with $J y_{j}^{\prime}-B y_{j}=A f_{j}$ a.e. on $[a, b)$ (for $j \in\{1,2\}$ ),

$$
f_{1}=f_{2} \quad \text { in } L_{A}^{2} \text {. }
$$

Proof. If we set $y:=y_{1}-y_{2}$, we obtain $A y=0$ a.e. on $[a, b)$.

Further we define $f:=f_{1}-f_{2}$. Therewith,

$$
J y^{\prime}=B y+A f=(\mu A+B) y+A f \text { a.e. on }[a, b),
$$

whence $y=R_{\mu} f+\Theta_{\mu} \xi_{1}+\Phi_{\mu} \xi_{2}$ by $(4.37)$, for some $\xi_{1} \in \mathbb{C}^{n^{-}}$and $\xi_{2} \in \mathbb{C}^{n^{+}}$.

Using (4.11) and (4.38), the boundary condition $\left[y, \chi_{\mu}\right](a)$ yields $\xi_{1}=0$, and so the boundary condition $\left[y, \zeta_{\mu}\right](b)$ implies $\xi_{2}=0$ since $\zeta_{\mu}=\eta_{\mu}+\chi_{\mu} M^{*}(\mu)$.

Thus we know $y=R_{\mu} f$. Since $y=0$ in $L_{A}^{2}$, Assumption 2 yields $f=0$ in $L_{A}^{2}$ and hence $f_{1}=f_{2}$ in $L_{A}^{2}$.

Now we can define

$$
\begin{gathered}
D(L):=\left\{y \in L_{A}^{2} \cap A C_{\mathrm{loc}}[a, b):\left[y, \chi_{\mu}\right](a)=0,\left[y, \zeta_{\mu}\right](b)=0,\right. \\
\quad \begin{array}{l}
\text { and there exists } \left.f \in L_{A}^{2}: J y^{\prime}-B y=A f \text { a.e. }\right\}, \\
L y:=f \quad \text { for } y \in D(L), f \in L_{A}^{2} \text { with } J y^{\prime}-B y=A f .
\end{array} \\
\begin{array}{c}
\text { and there exists } \left.f \in L_{A}^{2}: J z^{\prime}-B^{*} z=A f \text { a.e. }\right\}, \\
\widehat{L} z:=f \quad \text { for } z \in D(L), f \in L_{A}^{2} \text { with } J z^{\prime}-B^{*} z=A f .
\end{array}
\end{gathered}
$$

More precisely $D(L)$ and $\widehat{D}(L)$ consist of all equivalence classes in $L_{A}^{2}$ such that at least one representative of the class satisfies the conditions after the colons. Lemma 4.4.3 and an analogous statement for the adjoint problem ensure that $L$ and $\widehat{L}$ are well-defined.

Note that the boundary conditions at $a$ are independent of $\mu$, because so are $\chi_{\mu}(a)$ and $\Phi_{\mu}(a)$.

Lemma 4.4.4. ${ }^{* 1}$ For $\lambda \in W_{V}$ and $f \in L_{A}^{2}$ holds $R_{\lambda} f \in D(L)$ and $(L-\lambda) R_{\lambda} f=f$.

Proof. Theorem 4.3.3 implies $R_{\lambda} f \in L_{A}^{2}$.

Corollary 4.3.2 yields $R_{\lambda} f \in A C_{\text {loc }}$ and

$$
J\left(R_{\lambda} f\right)^{\prime}=B R_{\lambda} f+A\left(\lambda R_{\lambda} f+f\right) .
$$


Since $\chi_{\lambda}(a)=\chi_{\mu}(a)$, equation (4.38) shows that $R_{\lambda} f$ satisfies the boundary condition at $a$ required in $D(L)$.

For the second boundary condition we first assume $f$ has compact support.

Let $c$ large enough, such that $\operatorname{supp}(f) \subset[a, c]$. On $[c, b)$, equations (4.34) and (4.35) yield

$$
R_{\lambda} f=\Psi_{\lambda} \xi
$$

for some $\xi \in \mathbb{C}^{n^{-}}$. Thus, by (4.30),

$$
\left[R_{\lambda} f, \zeta_{\mu}\right](b)=\left[\Psi_{\lambda}, \zeta_{\mu}\right](b) \xi=0 .
$$

To obtain the boundary condition in the general case, we first calculate, for arbitrary $g \in L_{A}^{2}$,

$$
\begin{aligned}
{\left[R_{\lambda} g, \zeta_{\mu}\right]^{\prime} } & =\zeta_{\mu}^{*} J\left(R_{\lambda} g\right)^{\prime}-\left(J \zeta_{\mu}^{\prime}\right)^{*} R_{\lambda} g \\
& =\zeta_{\mu}^{*}\left((\lambda A+B) R_{\lambda} g+A g\right)-\left(\left(\bar{\mu} A+B^{*}\right) \zeta_{\mu}\right)^{*} R_{\lambda} g \\
& =(\lambda-\mu) \zeta_{\mu}^{*} A R_{\lambda} g+\zeta_{\mu}^{*} A g .
\end{aligned}
$$

Now let $f \in L_{A}^{2}$ and let $\left(f_{m}\right)$ a sequence of compact support functions converging to $f$ in $L_{A}^{2}$. Using (4.55) and (4.56), we obtain

$$
\begin{aligned}
{\left[R_{\lambda} f, \zeta_{\mu}\right](b)=} & {\left[R_{\lambda}\left(f-f_{m}\right), \zeta_{\mu}\right](b) } \\
= & {\left[R_{\lambda}\left(f-f_{m}\right), \zeta_{\mu}\right](a)+\int_{a}^{b}\left((\lambda-\mu) \zeta_{\mu}^{*} A R_{\lambda}\left(f-f_{m}\right)+\zeta_{\mu}^{*} A\left(f-f_{m}\right)\right) d x } \\
= & \zeta_{\mu}^{*}(a) J \Phi_{\lambda}(a) \int_{a}^{b} \zeta_{\lambda}^{*} A\left(f-f_{m}\right) d x+ \\
& (\lambda-\mu) \int_{a}^{b} \zeta_{\mu}^{*} A R_{\lambda}\left(f-f_{m}\right) d x+\int_{a}^{b} \zeta_{\mu}^{*} A\left(f-f_{m}\right) d x .
\end{aligned}
$$

Since $R_{\lambda}$ is bounded, $f-f_{m} \rightarrow_{L_{A}^{2}} 0$ implies $R_{\lambda}\left(f-f_{m}\right) \rightarrow_{L_{A}^{2}} 0$ for $m \rightarrow \infty$. Since $\zeta_{\mu} \in L_{A}^{2}$, the right-hand side of (4.57) tends to zero for $m \rightarrow \infty$. Hence $\left[R_{\lambda} f, \zeta_{\mu}\right](b)=0$.

In view of (4.54), we have shown $R_{\lambda} f \in D(L)$, and $L R_{\lambda} f=f+\lambda R_{\lambda} f$, and thus $(L-$ ג) $R_{\lambda} f=f$.

Lemma 4.4.5.*2 $D(L)$ and $D(\widehat{L})$ are dense in $L_{A}^{2}$.

Proof. Let $f \in L_{A}^{2}$ be orthogonal to $D(L)$. For all $g \in L_{A}^{2}$,

$$
<g, \widehat{R}_{\mu} f>=<R_{\mu} g, f>=0
$$


by Corollary 4.3.4 and Lemma 4.4.4. This shows $\widehat{R}_{\mu} f=0$ and hence $f=0$ by Assumption 2 .

With a corresponding argument, the denseness of $D(\widehat{L})$ follows.

We denote the resolvent set of of an operator by $\rho(\cdot)$.

We will see that $W_{V} \subseteq \rho(L)$, with $R_{\lambda}=(L-\lambda)^{-1}$ for $\lambda \in W_{V}$. We first show this assertion for $\lambda=\mu$ :

Lemma 4.4.6. ${ }^{*} \mu \in \rho(L)$ with $(L-\mu)^{-1}=R_{\mu}$, and $\bar{\mu} \in \rho(\widehat{L})$ with $(\widehat{L}-\bar{\mu})^{-1}=\widehat{R}_{\mu}$.

Proof. For $y \in D(L)$ with $(L-\mu) y=0$ holds $L y=\mu y$ and thus $J y^{\prime}=(\mu A+B) y$. This yields $y=\Theta_{\mu} \xi_{1}+\Phi_{\mu} \xi_{2}$ for some $\xi_{1} \in \mathbb{C}^{n^{-}}$and $\xi_{2} \in \mathbb{C}^{n^{+}}$.

The boundary condition for $y$ at $a$, together with (4.11), implies $\xi_{1}=0$.

Using $\zeta_{\mu}=\eta_{\mu}+\chi_{\mu} M_{0}^{*}$, the boundary condition at $b$ and (4.11) yield $\xi_{2}=0$, thus $y=0$.

Hence $L-\mu$ is one-to-one.

Let $f \in L_{A}^{2}$ and $y \in D(L)$. Lemma 4.4 .4 yields $R_{\mu} f \in D(L),(L-\mu) R_{\mu} f=f$, and

$$
(L-\mu) R_{\mu}(L-\mu) y=(L-\mu) y,
$$

which implies $R_{\mu}(L-\mu) y=y$.

Together we conclude: $(L-\mu): D(L) \rightarrow L_{A}^{2}$ is bijective and $R_{\mu}$ is its inverse, which is bounded by Theorem 4.3.3.

With a corresponding argument, the statement for $\widehat{L}$ and $\widehat{R}_{\mu}$ follows.

Lemma 4.4.6 and Corollary 4.3.4 yield $(\widehat{L}-\bar{\mu})^{-1}=\left((L-\mu)^{-1}\right)^{*}$, and thus

Corollary 4.4.7. ${ }^{* 1} L$ is the $L_{A^{-}}^{2}$ adjoint of $\widehat{L}$ and vice versa.

Now we are able to show

Theorem 4.4.8. ${ }^{*}{ }_{V} \subseteq \rho(L)$, and $R_{\lambda}=(L-\lambda)^{-1}$ for $\lambda \in W_{V}$. Analogously for the adjoint problem $\left\{\bar{\lambda}: \lambda \in W_{V}\right\} \subseteq \rho(\widehat{L})$, and $\widehat{R_{\lambda}}=(\widehat{L}-\bar{\lambda})^{-1}$ for $\lambda \in W_{V}$.

Proof. Let $\lambda \in W_{V}$.

For $f \in L_{A}^{2}$, the adjoint analog of Lemma 4.4.4 yields $\widehat{R_{\lambda}} f \in D(\widehat{L})$ and $(\widehat{L}-\bar{\lambda}) \widehat{R_{\lambda}} f=f$, whence in particular the range of $\widehat{L}-\bar{\lambda}$ is $L_{A}^{2}$.

Corollary 4.4.7 implies $(L-\lambda)^{*}=\widehat{L}-\bar{\lambda}$. Hence $L-\lambda$ is one-to-one. 
The rest of the proof is exactly the same as the second part of the proof of Lemma 4.4.6 with $\lambda$ instead of $\mu$.

Because their resolvent sets are not empty, we conclude

Corollary 4.4.9. ${ }^{*}$ L and $\widehat{L}$ are closed.

Now we take a look at the scalar examples on pages 23 to 30 .

From now on we assume for all scalar examples that $w>0$ on $[a, b)$.

We use Remark 4.4.1 to show that Assumption 2 is satisfied for all scalar examples.

Remark 4.4.1 poses assumptions only on $J, A$, and $B$, whence we need not distinguish different choices of $V$ and $E$.

We study the odd case with $m=1$ in detail. We have $A=A_{1}=\operatorname{diag}(w, 0,0), J=J_{1}=$ $\operatorname{codiag}(i, i, i)$ and

$$
B=B_{1}=\frac{1}{s}\left(\begin{array}{ccc}
-p_{0} & i q & 0 \\
i r & -\frac{p_{1}}{s} & -1 \\
0 & -1 & 0
\end{array}\right) .
$$

This leads to

$$
\begin{aligned}
& N_{1}=\operatorname{ker}(A)=\operatorname{span}\left\{e_{2}, e_{3}\right\}, \\
& N_{2}=N_{1} \cap B^{-1}\left(J N_{1}+\operatorname{range}(A)\right)=N_{1} \cap B^{-1}\left(\operatorname{span}\left\{e_{1}, e_{2}\right\}\right)=N_{1} \cap \operatorname{span}\left\{e_{1}, e_{3}\right\}=\operatorname{span}\left\{e_{3}\right\}, \\
& N_{3}=N_{2} \cap B^{-1}\left(J N_{2}+\operatorname{range}(A)\right)=\operatorname{span}\left\{e_{3}\right\} \cap B^{-1}\left(\operatorname{span}\left\{e_{1}\right\}\right)=\{0\} .
\end{aligned}
$$

The same calculation holds for $B^{*}$ in place of $B$. All requirements of Remark 4.4 .1 are fulfilled, hence Assumption 2 holds.

In the same way, for arbitrary $m$ and both cases (even and odd) and in the even case for both choices of $J\left(J_{1}\right.$ or $\left.J_{2}\right)$, with $n=2 m$, or $n=2 m+1$, respectively, we obtain

$$
N_{j}=\operatorname{span}\left\{e_{j+1}, \ldots e_{n}\right\}
$$

for $j<n$ and $N_{n}=\{0\}$. Hence Assumption 2 holds for all of our scalar examples by Remark 4.4.1.

Now we give some thoughts to the question in which cases the constructed operator $L$ is self-adjoint. Certainly, we cannot expect $L$ to be self-adjoint in the non-self-adjoint case $B \neq B^{*}$. But even in the self-adjoint case $B=B^{*}$, with the canonical choice of $V$, the resulting operator $L$ need not be self-adjoint.

Indeed, in this case, $L y=\widehat{L} y=L^{*} y$ for $y \in D(L) \cap D(\widehat{L})$. But the number of boundary 
conditions at $a$ for $L$ and $\widehat{L}$ may not coincide; in the odd case it is even impossible for them to coincide.

Furthermore, in the even case, also if $n^{+}=n^{-}$, the boundary conditions at a need not be the same. Straightforward calculations show that they are the same if and only if $E^{*} J E\left(\begin{array}{c}0 \\ I_{n^{+}}\end{array}\right)=\left(\begin{array}{c}\Xi \\ 0\end{array}\right)$ for some $\Xi \in \mathbb{C}^{n^{-}, n^{-}}$. By regularity of $J$ and $E$, this is equivalent to $\left(0 \mid I_{n^{+}}\right) E^{*} J E\left(\begin{array}{c}0 \\ I_{n^{+}}\end{array}\right)=0$. For the canonical choice $V= \pm i J$ this is finally equivalent to

$$
P_{\lambda}(a)=\frac{1}{2} \Phi_{\lambda}^{*}(a) V \Phi_{\lambda}(a)=0
$$

In our examples for the even case this does not hold for the suitable pairs $\left(V_{c}, E_{c}\right)$ and $\left(V_{c, 2}, E_{c, 2}\right)$, but it does hold for $\left(V_{c}, E_{s}\right)$.

The same boundary condition at $a$ still does not imply $L=\widehat{L}$. We will return to this subject at the end of this chapter.

Now we examine the set $W_{V}$, knowing that this is a subset of the resolvent set of the corresponding operator $L$.

In the self-adjoint setting with the canonical choice $V= \pm i J$, we already know that $W_{V}$ is the lower, respectively the upper halfplane.

In all our scalar examples we have $A=\operatorname{diag}(w, 0, \ldots, 0)$. The condition $-\boldsymbol{R e}(\lambda V J A) \succcurlyeq A$ for some $\lambda \in \mathbb{C}$ requires that the first column of $-V J$ is of the form $(\nu, 0, \ldots, 0)^{T}$ for some $\nu \in \mathbb{C} \backslash\{0\}$. (Otherwise $-\boldsymbol{R e}(\lambda V J A)$ would be indefinite or zero.) Thus,

$$
-\boldsymbol{R e}(\lambda V J A)=\boldsymbol{R e}(\lambda \operatorname{diag}(\nu w, 0, \ldots, 0))=\operatorname{diag}(\mathbf{R e}(\lambda \nu) w, 0, \ldots, 0)=\boldsymbol{\operatorname { R e }}(\lambda \nu) A,
$$

and therewith

$$
\Lambda_{V}=\{\lambda \in \mathbb{C}: \operatorname{Re}(\lambda \nu)>0\}
$$

which is a halfplane.

$W_{V}=\operatorname{Adm}_{V}+\Lambda_{V}$ is a (possibly) shifted halfplane. This holds, because $\Lambda_{V}$ is a halfplane, and therewith

$$
W_{V}=\bigcup_{\lambda \in \operatorname{Adm}_{V}}\left(\lambda+\Lambda_{V}\right)
$$

is a halfplane or the whole complex plane.

We exclude $W_{V}=\mathbb{C}$ as follows: Let $\nu$ again the first entry of $-V J$, we assume w.l.o.g $|\nu|=1$.

Since $\bar{\nu} \in \Lambda_{V}$, there exists some large $k>0$ such that $C_{0, V}+k \mathbf{R e}(\bar{v} V J A) \nsucc 0$. 
Let $\lambda \in \mathbb{C}$ with $\boldsymbol{\operatorname { R e }}(\lambda \nu) \leq-k$. We set $\lambda_{1}:=\operatorname{Re}(\lambda v) \bar{v}$ and $\lambda_{2}:=\lambda-\lambda_{1}$.

Therefore, $-\boldsymbol{R e}\left(\lambda_{2} V J A\right)=\boldsymbol{R e}\left(\lambda_{2} v\right) A=0$.

Using $\boldsymbol{R e}(\lambda v) \leq-k$ and $-\boldsymbol{R e}(\bar{v} V J A) \succcurlyeq 0$, we can calculate

$$
\begin{aligned}
C_{\lambda, V} & =C_{0, V}-\boldsymbol{\operatorname { R e }}(\lambda V J A) \\
& =C_{0, V}-\boldsymbol{\operatorname { R e }}\left(\lambda_{1} V J A\right)-\boldsymbol{\operatorname { R e }}\left(\lambda_{2} V J A\right) \\
& =C_{0, V}-\boldsymbol{\operatorname { R e }}(\lambda \nu) \operatorname{Re}(\bar{v} V J A)-0 \\
& \preccurlyeq C_{0, V}+k \mathbf{R e}(\bar{v} V J A) \nsucc 0 .
\end{aligned}
$$

Accordingly, $\lambda \notin \operatorname{Adm}_{V}$, whence $\lambda \notin W_{V}$ by (4.58).

\subsection{The maximal operator and deficiency indices}

This section mainly bases on Don Hinton's idea that the geometric shape of $D_{\lambda}(b)$ (in particular the quantity $\mathbf{r}$ defined on page 75 below) is related to the deficiency indices of the maximal operator (see $[17, \S 14-18]$ ).

In this section we are going to define the maximal operator for (2.1). The relation of its domain of definition to $D(L)$ provides a better understanding of the boundary conditions posed in $D(L)$.

We set

$$
\begin{aligned}
& D\left(L_{\text {max }}\right):=\left\{y \in L_{A}^{2} \cap A C_{\mathrm{loc}}[a, b): \text { there exists } f \in L_{A}^{2}: J y^{\prime}-B y=A f \text { a.e. }\right\} \\
& D\left(\widehat{L}_{\max }\right):=\left\{z \in L_{A}^{2} \cap A C_{\mathrm{loc}}[a, b): \text { there exists } f \in L_{A}^{2}: J z^{\prime}-B^{*} z=A f \text { a.e. }\right\}
\end{aligned}
$$

and

$$
\begin{aligned}
& D_{1}:=\left\{y \in D\left(L_{\max }\right):\left[y, \chi_{\mu}\right](a)=0\right\} \\
& \widehat{D}_{1}:=\left\{z \in D\left(\widehat{L}_{\max }\right):\left[z, \Phi_{\mu}\right](a)=0\right\} .
\end{aligned}
$$

As in the definition of $D(L)$ and $D(\widehat{L})$, these sets are meant as subsets of $L_{A}^{2}$. I.e. $y \in L_{A}^{2}$ is in one of these sets if and only if one representative of its class fulfills the required assumptions.

To define the maximal operator for (2.1) and for (3.15), we need one more

Assumption 3. For every $y \in A C_{\text {loc }}\left([a, b), \mathbb{C}^{n}\right)$ and $f \in L_{A}^{2}$, such that $y=0$ in $L_{A}^{2}$ and $y$ solves one of the equations $J y^{\prime}-B y=A f$ or $J y^{\prime}-B^{*} y=A f$ a.e. on $[a, b)$, holds $f=0$ in $L_{A}^{2}$. 
This assumption is equivalent to uniqueness of a corresponding function $f \in L_{A}^{2}$ for every $y \in D\left(L_{\max }\right)$ and every $z \in D\left(L_{\max }\right)$. Assumption 2 demands the same, but only for functions $y$, that fulfill the boundary conditions in $D(L)$, respectively in $D(\widehat{L})$. Hence Assumption 3 is stronger than Assumption 2.

Fortunately, the proofs of Remarks 4.4.1 and 4.4.2 (which give criteria that assure Assumption 2 to hold), only use, that $R_{\lambda} f$ fulfills $J\left(R_{\lambda} f\right)^{\prime}-B R_{\lambda} f=A f$ for some $f \in L_{A}^{2}$, to show $R_{\lambda} f=0$ in $L_{A}^{2} \Rightarrow f=0$ in $L_{A}^{2}$. It does not require the boundary conditions, that $R_{\lambda} f$ fulfills. Whence the same proofs, with $y \in D\left(L_{\max }\right)$ in place of $R_{\mu} f$, yield that the premises of these Remarks even imply Assumption 3. So we obtain

Corollary 4.5.1. If the premises of Remark 4.4.1 together with corresponding premises for the adjoint problem are fulfilled, or if the premises of Remark 4.4 .2 are fulfilled, then Assumption 3 holds true.

For the rest of this section we assume that Assumption 3 holds true.

\section{Lemma 4.5.2. ${ }^{* 3}$}

$$
\begin{aligned}
& D_{1}=D(L) \dot{+}\left\{\Phi_{\mu} \xi: \xi \in \mathbb{C}^{n^{+}} \text {and } \Phi_{\mu} \xi \in L_{A}^{2}\right\} \\
& \begin{aligned}
D\left(L_{\text {max }}\right) & =D_{1}+\left\{\Psi_{\mu} \xi: \xi \in \mathbb{C}^{n^{-}}\right\} \\
& =D(L) \dot{+}\left\{\Psi_{\mu} \xi_{1}+\Phi_{\mu} \xi_{2}: \xi_{1} \in \mathbb{C}^{n^{-}}, \xi_{2} \in \mathbb{C}^{n^{+}}, \text {and } \Phi_{\mu} \xi_{2} \in L_{A}^{2}\right\}
\end{aligned} \\
& \widehat{D}_{1}=D(\widehat{L}) \dot{+}\left\{\chi_{\mu} \xi: \xi \in \mathbb{C}^{n^{-}} \text {and } \chi_{\mu} \xi \in L_{A}^{2}\right\}, \\
& \begin{aligned}
D\left(\widehat{L}_{\text {max }}\right) & =D_{1}+\left\{\zeta_{\mu} \xi: \xi \in \mathbb{C}^{n^{+}}\right\} \\
& =D(L) \dot{+}\left\{\zeta_{\mu} \xi_{1}+\chi_{\mu} \xi_{2}: \xi_{1} \in \mathbb{C}^{n^{+}}, \xi_{2} \in \mathbb{C}^{n^{-}}, \text {and } \chi_{\mu} \xi_{2} \in L_{A}^{2}\right\},
\end{aligned}
\end{aligned}
$$

Proof. We only show (4.62), (4.63), and (4.64). The other equations can be proven analogously.

We first show (4.63). Therefor let $y \in D_{1}$ with corresponding $f \in L_{A}^{2}$ such that $J y^{\prime}-B y=$ Af a.e. on $[a, b)$, and let $\tilde{y}:=y+\Psi_{\mu} \xi$ for some $\xi \in \mathbb{C}^{n^{-}}$. Since $\Psi_{\mu}$ solves $(2.1)$ and every column of $\Psi_{\mu}$ is in $L_{A}^{2}$ by (4.7), we conclude that $\tilde{y}$ is in $D_{\max }$, with corresponding $\tilde{f}:=f+\mu \Psi_{\mu} \xi \in L_{A}^{2}$.

On the other hand, for arbitrary $y \in D\left(L_{\max }\right)$, we set $\xi:=\left[y, \chi_{\mu}\right](a)$. Using $\Psi_{\mu}=$ $\Theta_{\mu}+\Phi_{\mu} M_{0}$ (and hence $\left[\Psi_{\mu} \xi, \chi_{\mu}\right](a)=\xi$ by (4.11)) and the above knowledge again, we realize that $y-\Psi_{\mu} \xi$ is in $D_{1}$. This proves (4.63) 
Now we show (4.62), except that the sum is direct. This statement follows by (4.64). Therefor let $\tilde{y}=y+\Phi_{\mu} \xi \in L_{A}^{2}$ with $y \in D(L)$.

$D_{1}$ is obviously a superset of $D(L)$, thus it contains $y$.

By (4.11), $\left[\Phi_{\mu} \xi, \chi_{\mu}\right](a)=0$ and for $f:=\mu \Phi_{\mu} \xi \in L_{A}^{2}$ holds

$$
J \Phi_{\mu}^{\prime} \xi=A f+B \Phi_{\mu} \xi
$$

So $D_{1}$ contains $\Phi_{\mu} \xi$, too, and thus also $\tilde{y}$.

Now let $\tilde{y} \in D_{1}$, i.e. $\tilde{y} \in L_{A}^{2} \cap A C_{\text {loc }}[a, b)$ with $\left[\tilde{y}, \chi_{\mu}\right](a)=0$, and $f \in L_{A}^{2}$ such that $J \tilde{y}^{\prime}-B \tilde{y}=A f$.

Lemma 4.4.4 yields $y:=R_{\mu}(f-\mu \tilde{y}) \in D(L)$.

It suffices to show that $\varphi:=y-\tilde{y}=\Phi_{\mu} \xi$ for some $\xi \in \mathbb{C}^{n^{+}}$. Equation (4.37) yields

$$
J \varphi^{\prime}=(\mu A+B) y+A(f-\mu \tilde{y})-B \tilde{y}-A f=(\mu A+B) \varphi .
$$

Thus $\varphi=\Theta_{\mu} \xi_{1}+\Phi_{\mu} \xi_{2}$ for some $\xi_{1} \in \mathbb{C}^{n^{-}}, \xi_{2} \in \mathbb{C}^{n^{+}}$. Using $y \in D(L)$, we obtain

$$
\left[\varphi, \chi_{\mu}\right](a)=\left[y, \chi_{\mu}\right](a)-\left[\tilde{y}, \chi_{\mu}\right](a)=0
$$

which implies $\xi_{1}=0$ by (4.11). Hence $\varphi \in\left\{\Phi_{\mu} \xi: \xi \in \mathbb{C}^{n^{+}}\right.$and $\left.\Phi_{\mu} \xi \in L_{A}^{2}\right\}$.

It remains to show that the sum in (4.64) is direct.

So let $y:=\left(\Psi_{\mu} \mid \Phi_{\mu}\right) \xi \in D(L)$ for some $\xi \in \mathbb{C}^{n}$ (in particular $y \in L_{A}^{2}$ ). We have to show that $y=0$ in $L_{A}^{2}$.

We know, that $y$ solves $J y^{\prime}-B y=\mu A y$. Furthermore there exist some $\widetilde{y} \in L_{A}^{2}$ that vanishes in $L_{A}^{2}$, such that $y+\widetilde{y}$ fulfills the premises in the definition of $D(L)$. Whence there is some $f=L y$ with

$$
J(y+\widetilde{y})^{\prime}-B(y+\widetilde{y})=A f .
$$

Thus, for $\tilde{f}:=f-\mu y$, we obtain

$$
J \widetilde{y}^{\prime}-B \widetilde{y}=A \widetilde{f} .
$$

Assumption 3 yields $\widetilde{f}=0$ in $L_{A}^{2}$, whence $f=\mu y$ in $L_{A}^{2}$. So we obtain $L y=f=\mu y$. We know that $\mu$ is in the resolvent set of $L$. Thus we conclude $y=0$ in $L_{A}^{2}$.

Lemma 4.5.2 shows, in particular, that, if there is no $L_{A}^{2}$-function $\Phi_{\mu} \xi$ for some non-trivial $\xi \in \mathbb{C}^{n^{+}}$, then there are no boundary conditions in $D(L)$ at $b$ (i.e., $\left[y, \zeta_{\mu}\right](b)=0$ follows automatically from the other conditions posed in $D(L))$. Later we will call this the strict 
limit-point case.

We want to define the maximal operator for (2.1). With Assumption 3, the following operator is well-defined:

$$
L_{\text {max }}:\left\{\begin{array}{l}
D\left(L_{\max }\right) \rightarrow L_{A}^{2} \\
y \mapsto f \text { with } J y^{\prime}-B y=A f \text { a.e. on }[a, b) .
\end{array}\right.
$$

We call $L_{\max }$ the maximal operator for (2.1). Furthermore, for $\lambda \in \mathbb{C}$, let

$$
N_{\lambda}:=\operatorname{ker}\left(L_{\max }-\lambda\right)
$$

denote the deficiency space, and its dimension $d_{\lambda}$ the deficiency index of $L_{\max }$.

For $\xi_{1} \in \mathbb{C}^{n^{-}}$and $\xi_{2} \in \mathbb{C}^{n^{+}}$such that $\Phi_{\mu} \xi_{2} \in L_{A}^{2}$, we have $L_{\max }\left(\Psi_{\mu} \xi_{1}+\Phi_{\mu} \xi_{2}\right)=\mu\left(\Psi_{\mu} \xi_{1}+\right.$ $\left.\Phi_{\mu} \xi_{2}\right)$. Since $\mu$ is in the resolvent set of $L$, (4.64) yields

\section{Corollary 4.5.3.}

$$
N_{\mu}=\left\{\Psi_{\mu} \xi: \xi \in \mathbb{C}^{n^{-}}\right\}+\left\{\Phi_{\mu} \xi: \xi \in \mathbb{C}^{n^{+}} \text {and } \Phi_{\mu} \xi \in L_{A}^{2}\right\} \subseteq L_{A}^{2} .
$$

If only the trivial solution of (2.1) (with $\lambda=\mu$ ) vanishes in $L_{A}^{2}$, then, since $\left(\Psi_{\mu} \mid \Phi_{\mu}\right)$ has full rank,

$$
d_{\mu}=n^{-}+\operatorname{dim}\left\{\Phi_{\mu} \xi: \xi \in \mathbb{C}^{n^{+}} \text {and } \Phi_{\mu} \xi \in L_{A}^{2}\right\}
$$

$\mu \in W_{V}$ was chosen arbitrarily, furthermore the maximal operator and the deficiency indices do not depend on this choice. Thus we can conclude

Corollary 4.5.4. For every $\lambda \in W_{V}$,

$$
N_{\lambda}=\left\{\Psi_{\lambda} \xi: \xi \in \mathbb{C}^{n^{-}}\right\}+\left\{\Phi_{\lambda} \xi: \xi \in \mathbb{C}^{n^{+}} \text {and } \Phi_{\lambda} \xi \in L_{A}^{2}\right\} \subseteq L_{A}^{2} .
$$

If only the trivial solution of (2.1) vanishes in $L_{A}^{2}$, then

$$
d_{\lambda}=n^{-}+\operatorname{dim}\left\{\Phi_{\lambda} \xi: \xi \in \mathbb{C}^{n^{+}} \text {and } \Phi_{\lambda} \xi \in L_{A}^{2}\right\} .
$$

Now we consider the scalar examples. For the differential expressions $\tau_{e}[v]$ and $\tau_{o}[v]$ in $L_{w}^{2}$ on page 23 , with arbitrary coefficient functions in $L_{l o c}^{1}$, we define the maximal operators by

$$
\begin{aligned}
& D\left(L_{e}^{\max }\right):=\left\{v \in L_{w}^{2}(a, b): v^{[j]} \in A C_{\mathrm{loc}}[a, b) \text { for } 0 \leq j \leq 2 m-1\right. \\
& \text { and } \left.\tau_{e}[v]=w f \text { for some } f \in L_{w}^{2}(a, b)\right\} \text {, } \\
& L_{e}^{\max } v:=f \in L_{w}^{2} \quad \text { such that } \tau_{e}[v]=w f \text {, } \\
& D\left(L_{o}^{\max }\right):=\left\{v \in L_{w}^{2}(a, b): v^{[j]} \in A C_{\mathrm{loc}}[a, b) \text { for } 0 \leq j \leq 2 m\right. \\
& \text { and } \left.\tau_{o}[v]=w f \text { for some } f \in L_{w}^{2}(a, b)\right\} \text {, } \\
& L_{o}^{\max } v:=f \in L_{w}^{2} \quad \text { such that } \tau_{o}[v]=w f .
\end{aligned}
$$


Since $w>0$ on $[a, b)$, and $w f=w \tilde{f}$ implies $w(f-\tilde{f})=0$, we see that the maximal operators are well-defined.

We like to compare these maximal operators with the maximal operator for (2.1) with corresponding functions. If we choose for example $J=J_{1}$, we obtain $A=A_{1}=\operatorname{diag}(w, 0, \ldots, 0)$ and $B=B_{1}$ (see pages 24 to 25 for the even and pages 25 to 26 for the odd case. Again, as we will see, the actual choice of $J$ has no influence in the following.)

We have shown that Assumption 2 holds true by showing the premises of Remark 4.4.1. Hence Corollary 4.5.1 yields, that also Assumption 3 holds true.

Furthermore, since $w>0$ on $[a, b)$, the following shows that only the trivial solution of (2.1) vanishes in $L_{A}^{2}$ : Let $Y=\left(v_{1}, \ldots, v_{n}\right)^{T}$ a solution of $J Y^{\prime}=\left(\lambda A_{1}+B_{1}\right)$ with $A_{1} Y=0$ a.e. on $[a, b)$. Then $v_{1}$ is a solution of one of the scalar equations (2.31), respectively (2.32). Furthermore $w v_{1}=0$ a.e. on $[a, b)$ (whence, by continuity, $v_{1}=0$ ) and $v_{j}=v_{1}^{[j-1]}=0$ for $j \leq n$. Thus follows $Y=0$.

For simplicity, now we only consider the even case. The following results hold correspondingly for the odd case with analog proofs.

Let $L_{\text {max }}$ the maximal operator for $J_{1} Y^{\prime}=\left(\lambda A_{1}+B_{1}\right) Y$.

For functions $f, g \in L_{A}^{2}$ let $f_{1}, g_{1}$ denote the first components. We have

$$
<f, g>_{L_{A}^{2}}=\int_{a}^{b} g^{*} A f d x=\int_{a}^{b} g_{1} w f_{1} d x=<f_{1}, g_{1}>_{L_{w}^{2}} .
$$

This shows that $\Pi: L_{A}^{2} \rightarrow L_{w}^{2}, f \mapsto f_{1}$ is well-defined and isometric. Obviously $\Pi$ is also surjective and therewith bijective.

Lemma 4.5.5. $\Pi$ maps $L_{\max }$ to $L_{e}^{\max }$, i.e. $\Pi L_{\max } \Pi^{-1}=L_{e}^{\max }$. (The corresponding statement for the odd case can be shown analogously.)

Proof. For $y \in D\left(L_{\max }\right)$ let $f:=L_{\max } y$. By definition, we know that $y$ and $f$ are in $L_{A}^{2}$, hence $v:=y_{1}=\Pi y$ and $f_{1}=\Pi f$ are in $L_{w}^{2}$. Furthermore each component of $y$ is in $A C_{\text {loc }}[a, b)$ and it holds $J_{1} y^{\prime}=B_{1} y+A_{1} f$. By multiplication with $-J_{1}$ we obtain

$$
y^{\prime}=M_{B}^{e} y+M_{A}^{e} f=M_{B}^{e} y+\left(0 \cdots 0-w f_{1}\right)^{T} .
$$

By construction of $M_{B}^{e}$ and $M_{A}^{e}$ on pages 23 to $25,(4.70)$ is equivalent to $y_{j}=y_{1}^{[j-1]}=v^{[j-1]}$ for $2 \leq j \leq 2 m$ and $\tau_{e}\left[y_{1}\right]=w f_{1}$.

Hence all quasi-derivatives of $v$ are in $A C_{\mathrm{loc}}$, and $\tau_{e}[v]=\tau_{e}\left[y_{1}\right]=w f_{1}$. Thus we conclude that $\Pi y=v \in D\left(L_{e}^{\max }\right)$ and

$$
L_{e}^{\max }(\Pi y)=L_{e}^{\max } v=f_{1}=\Pi f=\Pi L_{\max } y
$$


Since $\Pi$ is injective, it remains to show that $\Pi$ maps $D\left(L_{\max }\right)$ onto $D\left(L_{e}^{\max }\right)$. For $v \in D\left(L_{e}^{\max }\right)$, we set $y:=\left(v^{[j-1]}\right)_{1 \leq j \leq 2 m}$ and $f:=\left(L_{e}^{\max } v, 0, \ldots, 0\right)^{T}$. Using (4.70) again, we see that $y \in D\left(L_{\max }\right)$, furthermore $\Pi y=v^{[0]}=v$. Thus

$$
\Pi\left(D\left(L_{\max }\right)\right)=D\left(L_{e}^{\max }\right)
$$

We conclude that the deficiency index of $L_{\max }$ is equal to that of $L_{e}^{\max }$.

Now we consider again the self-adjoint even order Example $\mathbf{1 . 2}$ on page 27, with the suitable pair $\left(V_{c}, E_{s}\right)$. This is the self-adjoint example with canonical choice of $V=V_{c}$, $n^{+}=n^{-}$, and $E=E_{s}$ is chosen such that the corresponding operators $L$ and $\widehat{L}$ have the same boundary condition at $a\left(\right.$ since $\left.P_{\lambda}(a)=0\right)$. Thus we have $D_{1}=\widehat{D}_{1}$.

If we furthermore assume that $\Phi_{\mu} \xi_{1}, \chi_{\mu} \xi_{2} \in L_{A}^{2}$ for some $\xi_{1} \in \mathbb{C}^{n^{+}}, \xi_{2} \in \mathbb{C}^{n^{-}}$implies $\Phi_{\mu} \xi_{1}=$ $\chi_{\mu} \xi_{2}=0$ in $L_{A}^{2}$, then (4.62) and (4.65) yield $D(L)=D_{1}=\widehat{D}_{1}=D(\widehat{L})$. This would mean $L=\widehat{L}=L^{*}$.

In particular $d_{\bar{\mu}}=d_{\mu}=n^{-}$implies $L=L^{*}$. (Since $B$ is self-adjoint in this example, $d_{\bar{\mu}}$ is the deficiency index of the maximal operator for the adjoint problems.) 


\section{Chapter 5}

\section{Results}

This chapter contains various results.

Relations between the geometrical shape of $D_{\lambda}(b)-C_{\lambda}(b)$ and the dimensions of the solution spaces of (2.1) within certain Hilbert spaces are examined. According to those quantities we introduce some limit-point and limit-circle type classification.

Properties of the $M$-function may yield further information about the spectrum of $L$.

With the aim to obtain yet further estimations for the spectrum of $L$, we restrict (2.1) to some smaller interval $[\tilde{a}, b) \subset[a, b)$, furthermore we vary the choice of an admissible matrix $V$.

\subsection{Dimensions of the solution spaces}

In this section we examine connections between the shape of $D_{\lambda}(b)-C_{\lambda}(b)$ and the dimension of the solution spaces of (2.1) and (3.15), respectively, intersected with $L_{A}^{2}$ or with $L_{C_{\lambda}}^{2}$, respectively with $L_{\widehat{C_{\lambda}}}^{2}$.

We will recover the two common cases of limit-point and limit-circle. But, since we consider the higher order and non-self-adjoint situation, these cases require a more sophisticated distinction.

Every statement and definition in this section, formulated for problem (2.1), also holds correspondingly for the adjoint problem. Recall the exchanged roles of $n^{+}$and $n^{-}$there.

For $F \in\left\{A, C_{\lambda}\right\}, m \in \mathbb{N}_{0}$ and any matrix-valued function $\Upsilon:[a, b) \rightarrow \mathbb{C}^{n, m}$ we use the notation

$$
[\Upsilon]_{F}:=\left\{\Upsilon \xi: \xi \in \mathbb{C}^{m} \text { and } \Upsilon \xi \in L_{F}^{2}\right\}
$$


Lemma 5.1.1. ${ }^{* 2}$ For $\lambda \in W_{V}, F \in\left\{A, C_{\lambda}\right\}$ let

$$
m_{F}:=\operatorname{dim}\left[\Phi_{\lambda}\right]_{F} .
$$

There are exactly $n^{-}+m_{F}$ linearly independent solution of (2.1) which lie in $L_{F}^{2}$.

Proof. From

$$
\Psi_{\lambda}=Y_{\lambda}\left(\begin{array}{c}
I_{n^{-}} \\
M(\lambda)
\end{array}\right)
$$

we see that $\Psi_{\lambda}$ has rank $n^{-}$. The columns of $\Psi_{\lambda}$ are in $L_{C_{\lambda}}^{2}$ and in $L_{A}^{2}$ by (4.6) and (4.7). Let $F \in\left\{A, C_{\lambda}\right\}$. Using $\Phi_{\lambda}=Y_{\lambda}\left(\begin{array}{c}0 \\ I_{n^{+}}\end{array}\right)$, with (5.1) we conclude the directness of the sum $\left[\Psi_{\lambda}\right]_{F} \dot{+}\left[\Phi_{\lambda}\right]_{F}$. Thus this space, consisting of functions in $L_{F}^{2}$, has dimension $n^{-}+m_{F}$. Therefore, with $n^{-}+m$ denoting the exact number of linearly independent solutions of (2.1) in $L_{F}^{2}$, we have $m \geq m_{F}$.

On the other hand, $n^{-}+m$ independent solutions of (2.1) are of the form $Y_{\lambda} \Xi$ with a matrix $\Xi \in \mathbb{C}^{n, n^{-}+m}=\mathbb{C}^{n^{-}+n^{+}, n^{-}+m}$ of full rank $n^{-}+m$. W.l.o.g. let $\Xi$ a block matrix of the shape

$$
\left(\begin{array}{cc}
\Xi_{1} & 0_{n^{-}, m} \\
\Xi_{2} & \Xi_{3}
\end{array}\right)
$$

with $\Xi_{3} \in \mathbb{C}^{n^{+}, m}$. This implies that every column of $\Phi_{\lambda} \Xi_{3}$ lies in $\left[\Phi_{\lambda}\right]_{F}$. Thus $m_{F} \geq m$.

Lemma 5.1.2. ${ }^{*_{2}}$ For $\lambda \in W_{V}$ let $m$ the number of eigenvalues of $P_{\lambda}(c)$ that remain bounded as $c \rightarrow b$.

There are exactly $n^{-}+m$ linearly independent solutions of $(2.1)$ which lie in $L_{C_{\lambda}}^{2}$.

Proof. If there are $n^{-}+m$ linearly independent solution of (2.1) which lie in $L_{C_{\lambda}}^{2}$, by Lemma 5.1.1 there exists $\Xi \in \mathbb{C}^{n, m}$ with rank $m$, such that the columns of $\Phi_{\lambda} \Xi$ are in $L_{C_{\lambda}}^{2}$. Using $(2.20)$ and $(2.13)$, we obtain for every $\xi \in \mathbb{C}^{m}$

$$
\xi^{*} \Xi^{*} P_{\lambda}(c) \Xi \xi=\xi^{*} \Xi^{*} P_{\lambda}(a) \Xi \xi+\int_{a}^{c}\left(\Phi_{\lambda} \Xi \xi\right)^{*} C_{\lambda} \Phi_{\lambda} \Xi \xi d x \leq \xi^{*} \Xi^{*} P_{\lambda}(a) \Xi \xi+\left\|\Phi_{\lambda} \Xi \xi\right\|_{C_{\lambda}}^{2} .
$$

The space $\left\{\Xi \xi: \xi \in \mathbb{C}^{m}\right\}$ has dimension $m$. Hence the min-max-principle yields that the $m$ smallest eigenvalues of $P_{\lambda}(c)$ remain bounded as $c$ tends to $b$.

Conversely, let $0 \leq \lambda_{1}(c) \leq \lambda_{2}(c) \leq \cdots \leq \lambda_{m}(c)$ the smallest eigenvalues of $P_{\lambda}(c)$ with corresponding orthonormal eigenvectors $\xi_{1}(c), \ldots, \xi_{m}(c)$. We set $\Upsilon:=\left(\xi_{1}\left|\xi_{2}\right| \ldots \mid \xi_{m}\right)$. Since $\mathbb{C}^{n, m}$ has a compact unit sphere, there exists some increasing sequence $\left(c_{p}\right)$ tending to $b$ such that $\Upsilon\left(c_{p}\right)$ converges to some $\Xi \in \mathbb{C}^{n, m}$ for $p \rightarrow \infty$. The columns of $\Xi$ are orthonormal, thus $\Xi$ has rank $m$. 
If $m$ eigenvalues of $P_{\lambda}$ are bounded, then $\lambda_{m} \leq k$ on $[a, b)$ for some constant $k$. Using $P_{\lambda}(a) \geq 0$ and (2.20) again, this yields for any $p, \widetilde{p} \in \mathbb{N}$ with $p \geq \widetilde{p}$ :

$$
\begin{aligned}
\left(\Upsilon\left(c_{p}\right)\right)^{*} \int_{a}^{c_{\widetilde{p}}} \Phi_{\lambda}^{*} C_{\lambda} \Phi_{\lambda} d x \Upsilon\left(c_{p}\right) & \leq\left(\Upsilon\left(c_{p}\right)\right)^{*} \int_{a}^{c_{p}} \Phi_{\lambda}^{*} C_{\lambda} \Phi_{\lambda} d x \Upsilon\left(c_{p}\right) \\
& =\left(\Upsilon\left(c_{p}\right)\right)^{*} P_{\lambda}\left(c_{p}\right) \Upsilon\left(c_{p}\right)-\Upsilon\left(c_{p}\right)^{*} P_{\lambda}(a) \Upsilon\left(c_{p}\right) \\
& \leq k\left(\Upsilon\left(c_{p}\right)\right)^{*} \Upsilon\left(c_{p}\right)=k I_{m}
\end{aligned}
$$

Convergence of $\Upsilon\left(c_{p}\right)$ to $\Xi$ yields

$$
\int_{a}^{c_{\widetilde{p}}}\left(\Phi_{\lambda}(x) \Xi\right)^{*} C_{\lambda}(x) \Phi_{\lambda}(x) \Xi d x=\Xi^{*} \int_{a}^{c_{\widetilde{p}}} \Phi_{\lambda}^{*} C_{\lambda} \Phi_{\lambda} d x \Xi \leq k I_{m},
$$

for any $\widetilde{p} \in \mathbb{N}$. With $c_{\widetilde{p}} \rightarrow b($ as $\widetilde{p} \rightarrow \infty)$ and $C_{\lambda} \succcurlyeq 0$, we conclude $\Phi_{\lambda} \Xi \in L_{C_{\lambda}}^{2}$.

Note that Lemmata 5.1.1 and 5.1.2 do not state that the solutions are linearly independent within $L_{C_{\lambda}}^{2}$ or $L_{A}^{2}$, respectively. The number of linearly independent solutions of (2.1) within $L_{C_{\lambda}}^{2}$, or within $L_{A}^{2}$, respectively, may be smaller.

For $\lambda \in W_{V}$ we define the geometrical variables

$$
\begin{gathered}
\mathcal{L}_{\lambda}:=D_{\lambda}(b)-C_{\lambda}(b), \\
\mathbf{r}:=\operatorname{dim}\left(\operatorname{span}\left(\left\{\boldsymbol{\Xi} \xi: \boldsymbol{\Xi} \in \mathcal{L}_{\lambda}, \xi \in \mathbb{C}^{\mathbf{n}^{-}}\right\}\right)\right) .
\end{gathered}
$$

Theorem 5.1.3. ${ }^{* 1}$ Let $\lambda \in W_{V}$.

(i) There are at least $n^{-}+\mathbf{r}$ linearly independent solutions of $(2.1)$ which lie in $L_{C_{\lambda}}^{2}$.

(ii) If $\mathcal{R}_{\lambda}(x) \nrightarrow 0$ as $x \rightarrow b$, there are exactly $n^{-}+\mathbf{r}$ linearly independent solutions of $(2.1)$ which lie in $L_{C_{\lambda}}^{2}$.

Proof. The proof is completely analogous to the proof of [5, Theorem 4.2 and Lemma 4.3], with the exception that $n$ must be replaced by $n^{-}$. The required corresponding assertions are Theorem 2.2.7, Lemma 5.1.2, Corollary 2.2.3, (2.26) and (2.27).

Lemmata 5.1.1, 5.1.2 and Theorem 5.1.3 together yield 
Corollary 5.1.4. Let $\lambda \in W_{V}$.

The number of linearly independent solutions of (2.1) which lie in $L_{C_{\lambda}}^{2}$, minus $n^{-}$, is equal to $\operatorname{dim}\left[\Phi_{\lambda}\right]_{C_{\lambda}}$ and equal to the number of bounded eigenvalues of $P_{\lambda}$.

If $\mathcal{R}_{\lambda}(x) \nrightarrow 0$ as $x \rightarrow b$, then it is also equal to $\mathbf{r}$.

For further conclusions we need the following topological properties of $\operatorname{Adm}_{V}$ and $\Lambda_{V}$ (see $(2.5)$ and $(2.6))$.

Lemma 5.1.5. Adm $m_{V}$ is convex, $\Lambda_{V}$ is an open wedge. Thus $W_{V}=A d m_{V}+\Lambda_{V}$ is open and convex. Furthermore $W_{V}$ is the set of the interior points of $A d m_{V}$.

Proof. Let $k_{1}, k_{2} \in \operatorname{Adm}_{V}$ and $\varrho \in(0,1)$. Definition (2.5) yields

$$
-\boldsymbol{R e}\left(k_{j} V J A\right) \geq \boldsymbol{R e}(V J B) \quad \text { a.e. on }[a, b)
$$

for $j=1,2$. Thus for $k:=\varrho k_{1}+(1-\varrho) k_{2}$, we obtain

$$
-\boldsymbol{\operatorname { R e }}(k V J A) \geq \varrho \mathbf{R e}(V J B)+(1-\varrho) \boldsymbol{R e}(V J B)=\boldsymbol{\operatorname { R e }}(V J B) \quad \text { a.e. on }[a, b),
$$

which means $k \in \operatorname{Adm}_{V}$. Hence $\operatorname{Adm}_{V}$ is convex.

Let $\lambda_{1}, \lambda_{2} \in \Lambda_{V}$ with corresponding $\delta_{1}, \delta_{2}>0$ from Definition (2.6) such that

$$
-\boldsymbol{R e}\left(\lambda_{j} V J A\right) \geq \delta_{j} A \quad \text { a.e. on }[a, b)
$$

for $j=1,2$. Furthermore let $\varrho \in(0,1)$. For $\lambda:=\varrho \lambda_{1}+(1-\varrho) \lambda_{2}$ and $\delta:=\min \left\{\delta_{1}, \delta_{2}\right\}$ we obtain

$$
-\boldsymbol{R e}(\lambda V J A) \geq \varrho \delta_{1} A+(1-\varrho) \delta_{2} A \geq \varrho \delta A+(1-\varrho) \delta A=\delta A .
$$

Thus we know $\lambda \in \Lambda_{V}$, which shows convexity of $\Lambda_{V}$.

For $\varrho>0$ and $\lambda \in \Lambda_{V}$,

$$
-\boldsymbol{R e}(\varrho \lambda V J A)=\varrho(-\boldsymbol{\operatorname { R e }}(\lambda V J A)) \succcurlyeq-\boldsymbol{R e}(\lambda V J A) \succcurlyeq A
$$

shows $\varrho \lambda \in \Lambda_{V}$.

Finally let $\lambda \in \Lambda_{V}$ with corresponding $\delta>0$, such that $-\boldsymbol{R e}(\lambda V J A) \geq \delta A$ a.e. on $[a, b)$. By Corollary 4.1.2 $(v)$ there exists $\varepsilon>0$ with

$$
A \geq \pm \varepsilon \operatorname{Re}(V J A), \quad A \geq \pm \varepsilon \operatorname{Re}(i V J A) \quad \text { a.e. on }[a, b) .
$$

Thus for $\nu \in \mathbb{C}$ with $|\nu-\lambda|<\frac{1}{3} \delta \varepsilon$ we have

$$
\begin{aligned}
-\boldsymbol{R e}(\nu V J A) & \geq-\boldsymbol{R e}(\nu V J A)-(-\mathbf{R e}(\lambda V J A)-\delta A) \\
& =-\boldsymbol{R e}((\nu-\lambda) V J A)+\delta A \\
& =-\boldsymbol{R e}(\nu-\lambda) \mathbf{R e}(V J A)-\mathbf{I m}(\nu-\lambda) \mathbf{R e}(i V J A)+\delta A \\
& \geq-\frac{1}{3} \delta \varepsilon \frac{1}{\varepsilon} A-\frac{1}{3} \delta \varepsilon \frac{1}{\varepsilon} A+\delta A=\frac{1}{3} \delta A \quad \text { a.e. on }[a, b) .
\end{aligned}
$$


Hence $\nu \in \Lambda_{V}$, which shows that $\Lambda_{V}$ is open.

We have shown that $\operatorname{Adm}_{V}$ is convex and $\Lambda_{V}$ is an open wedge. Thus $W_{V}=\operatorname{Adm}_{V}+\Lambda_{V}$ is open and convex.

Since $W_{V} \subseteq \operatorname{Adm}_{V}$, by (2.14), we obtain that every element of the open set $W_{V}$ is an interior point of $\mathrm{Adm}_{V}$.

On the other hand, let $\lambda$ an interior point of $\operatorname{Adm}_{V}$. Let $\varepsilon>0$, such that an $\varepsilon$-neighborhood of $\lambda$ lies in $\operatorname{Adm}_{V}$. We chosen some $\lambda_{0} \in \Lambda_{V}$ with $\left|\lambda_{0}\right|<\varepsilon$. Therewith $\lambda-\lambda_{0} \in \operatorname{Adm}_{V}$, whence, by definition, $\lambda=\lambda-\lambda_{0}+\lambda_{0} \in \operatorname{Adm}_{V}+\Lambda_{V}=W_{V}$.

Now we can conclude

Theorem 5.1.6. ${ }^{* 3}$ Let $N_{A}(\lambda), N_{C_{\lambda}}(\lambda)$ the number of linearly independent solutions of (2.1) that are in $L_{A}^{2}$ or in $L_{C_{\lambda}}^{2}$, respectively. Then $N_{A}$ and $N_{C_{\lambda}}$ are constant on $W_{V}$.

Proof. Let $F \in\left\{A, C_{\lambda}\right\}$.

By Lemma 5.1.5, the set $W_{V}$ is convex and open. Therewith it suffices to show that $N_{F}$ is locally constant. The assertion then follows by standard connectivity arguments.

Let $\lambda_{0} \in W_{V}$. By Theorem 4.3.3 there exists some $k, \varepsilon_{0}>0$, such that $\left\|R_{\lambda}\right\|_{F} \leq k$ for all $\lambda \in W_{V}$ with $\left|\lambda_{0}-\lambda\right|<\varepsilon_{0}$.

Let $\varepsilon:=\min \left\{\varepsilon_{0}, \frac{1}{k}\right\}>0$.

Furthermore let $\lambda_{1}, \lambda_{2} \in W_{V}$ with $\lambda_{1} \neq \lambda_{2}$ and $\left|\lambda_{0}-\lambda_{j}\right|<\varepsilon$ for $j=1,2$.

By symmetry it suffices to show $N_{F}\left(\lambda_{1}\right) \leq N_{F}\left(\lambda_{2}\right)$.

We set $m:=N_{F}\left(\lambda_{1}\right)-n^{-}$. We assume w.l.o.g. $m>0$, for otherwise the assertion follows by Lemma 5.1.1.

The same Lemma yields some matrix $\Xi \in \mathbb{C}^{n^{-}, m}$ with rank $m$ such that the columns of $\Phi_{\lambda_{1}} \Xi$ are in $L_{F}^{2}$. With

$$
J\left(\Phi_{\lambda_{1}} \Xi\right)^{\prime}=\left(\lambda_{2} A+B\right) \Phi_{\lambda_{1}} \Xi+\left(\lambda_{1}-\lambda_{2}\right) A \Phi_{\lambda_{1}} \Xi
$$

and (4.37), we obtain

$$
\Phi_{\lambda_{1}} \Xi=\left(\lambda_{1}-\lambda_{2}\right) R_{\lambda_{2}}\left(\Phi_{\lambda_{1}} \Xi\right)+\Theta_{\lambda_{2}} \Omega_{1}+\Phi_{\lambda_{2}} \Omega_{2}
$$

for some matrices $\Omega_{1} \in \mathbb{C}^{n^{-}, m}, \Omega_{2} \in \mathbb{C}^{n^{+}, m}$. Multiplication with $\chi_{\lambda_{1}}^{*} J$ from the left and evaluation at $a$ shows $\Omega_{1}=0$ by (4.11), (4.38), and $\chi_{\lambda_{1}}(a)=\chi_{\lambda_{2}}(a)$.

Hence the columns of $\Phi_{\lambda_{2}} \Omega_{2}$ are in $L_{F}^{2}$, because so are the columns of all other terms in (5.5) by assumption on $\Xi$ and Theorem 4.3.3. 
If $\Omega_{2}$ would have rank less than $m$, some nontrivial $\xi \in \mathbb{C}^{m}$ would exist with $\Omega_{2} \xi=0$. Whereas $\Xi \xi \neq 0$ since $\Xi$ has rank $m$. With (5.5) we could calculate

$$
R_{\lambda_{2}}\left(\Phi_{\lambda_{1}} \Xi \xi\right)=R_{\lambda_{2}}\left(\Phi_{\lambda_{1}} \Xi\right) \xi=\frac{1}{\lambda_{1}-\lambda_{2}} \Phi_{\lambda_{1}} \Xi \xi
$$

This would imply $\left\|R_{\lambda_{2}}\right\|_{F} \geq\left(\lambda_{1}-\lambda_{2}\right)^{-1}>k$, a contradiction.

Thus $\Omega_{2}$ has full rank. This shows that $\operatorname{dim}\left[\Phi_{\lambda_{2}}\right]_{F} \geq m$. Hence Lemma 5.1.1 yields $N_{F}\left(\lambda_{2}\right) \geq n^{-}+m=N_{F}\left(\lambda_{1}\right)$.

Since the number of linearly independent solutions of (2.1) that are in $L_{A}^{2}$ does not depend on the choice of a suitable pair, we obtain

Corollary 5.1.7. ${ }^{* 2}$ The number of linearly independent solutions of (2.1) that are in $L_{A}^{2}$ is constant on every connected component of the open set

$$
\bigcup_{\widetilde{V} \text { admissible }} W_{\widetilde{V}} .
$$

Now we want to classify the problem. Therefor let $\lambda \in W_{V}$.

In view of Theorem 5.1.6, Theorem 5.1.10, Lemma 5.1.12, and Theorem 5.1.14 below, in the following classifications there is no dependence on the actual choice of this $\lambda$ in $W_{V}$. Thus we do not note any dependences on $\lambda$. Yet it should be remarked, that the classification may depend on $V$.

We say, that problem (2.1) is in the limit-point case, if $\mathcal{L}_{\lambda}=\{0\}$. That is equivalent to $D_{\lambda}(b)$ consisting of exactly one point, respectively to $\mathbf{r}$ being zero.

The occurrence of an intermediate case, neither limit-point nor limit-circle is well known (e.g. see [16]). This naturally arises in higher order systems. $\mathcal{L}_{\lambda} \neq\{0\}$ does not imply that $\mathcal{L}_{\lambda}$ is an open set in $\mathbb{C}^{n^{+}, n^{-}}$(as long as $n^{+} n^{-}>1$ ).

Therefore we say that problem (2.1) is in the non-limit-point case, if it is not in the limitpoint case. To emphasis the geometrical role of $\mathbf{r}$ (which is at least 1 in the non-limit-point case), we also call it the r-non-limit-point case.

For the $n^{+}$-non-limit-point case we use the notation limit-circle case. Theorem 5.1.10 below shows that (2.1) is in the limit-circle case, if and only if all solutions of (2.1) are in $L_{C_{\lambda}}^{2}$.

An even stronger geometric assumption on $D_{\lambda}(b)$ is to have interior points within $\mathbb{C}^{n^{+}, n^{-}}$ and $n^{+} n^{-}>0$. We name this the full limit-circle case. 
If $\mathbf{r}<n^{+}$, then there may exist more than $n^{-}+\mathbf{r}$ linearly independent solution of (2.1) that are in $L_{A}^{2}$. There are three reasons for this.

First of all $L_{A}^{2}$ may be larger than $L_{C_{\lambda}}^{2}$, while the behavior of $D_{\lambda}(x)$, and therewith the value of $\mathbf{r}$, depends on $C_{\lambda}$ rather than on $A$. Already 1957, Sims observed this phenomenon in the two-dimensional case in [19].

The second reason is the freedom in $n^{+}$and $n^{-}$. For example if $n^{-}=0$, only the limit-point case can occur because $D_{\lambda}(b) \subset \mathbb{C}^{n^{+}, n^{-}}$, where $\mathbb{C}^{n^{+}, n^{-}}$is a vector space of dimension zero. Yet there may exist solutions of $(2.1)$ in $L_{A}^{2}$ (for an example see section 6.4 below). Thus, also if $A \asymp C_{\lambda}$ (e.g. in the self-adjoint case with canonical choice of $V$ ), there could exist more than $n^{-}$linearly independent solution of (2.1) that are in $L_{A}^{2}$.

The third reason is the following: As it will turn out, if $\mathcal{R}_{\lambda}$ tends to 0 , then $\mathbf{r}=\mathbf{0}$. But the amount of linearly independent solutions of (2.1) that are in $L_{A}^{2}$ depends on the behavior of $P_{\lambda}$ and may be larger than $n^{+}$(cp. Theorem $5.1 .3(i i)$ ).

To distinguish further characteristics of the problem (which are mainly of importance if $A \nsucc C_{\lambda}$ ), we also use the following classifications:

If there are exactly $n^{-}$independent solutions of (2.1) that are in $L_{A}^{2}$, we say that problem (2.1) is in the strict limit-point case.

We say that problem (2.1) is in the weak limit-circle case, if all solutions of (2.1) are in $L_{A}^{2}$.

Since $C_{\lambda} \succcurlyeq A$, the limit-circle case implies the weak limit-circle case, and, by definition, the non-limit-point case.

If $A \asymp C_{\lambda}$ and $n^{+} n^{-}>0$, then the weak limit-circle case is equivalent to the limit-circle case.

In the following, we use the notation $n^{+}(\cdot)$ for the number of positive eigenvalues of a self-adjoint matrix counted in their algebraic multiplicity.

Theorem 5.1.6 yields

Corollary 5.1.8. If (2.1) is in the weak limit-circle case, then this holds for every suitable $\operatorname{pair}(\widetilde{V}, \widetilde{E})$ with $W_{V} \cap W_{\widetilde{V}} \neq \emptyset$.

If (2.1) is in the strict limit-point case, then this holds for every suitable pair $(\widetilde{V}, \widetilde{E})$ with $W_{V} \cap W_{\widetilde{V}} \neq \emptyset$ and $n^{-}(\widetilde{V})=n^{-}$.

Furthermore holds

Lemma 5.1.9.*3 If $\mathcal{R}_{\lambda}(x) \rightarrow 0$ as $x \rightarrow b$, then $(2.1)$ is in the limit-point case.

Proof. Let $l \in D_{\lambda}(b)-C_{\lambda}(b)$. We show that $l=0 \in \mathbb{C}^{n^{+}, n^{-}}$, which gives $\mathcal{L}_{\lambda}=\{0\}$. 
Theorem 2.2.7 (iv) yields $l \in D_{\lambda}(c)-C_{\lambda}(c)$ for each $c \in\left[c_{0}(\lambda), b\right)$. By (2.26) this means $l^{*} P_{\lambda}(c) l \leq \mathcal{R}_{\lambda}(c)$.

Using Lemma 2.2.4 we obtain

$$
l^{*} P_{\lambda}\left(c_{0}(\lambda)\right) l \leq l^{*} P_{\lambda}(x) l \leq \mathcal{R}_{\lambda}(x)
$$

for every $x \in\left[c_{0}(\lambda), b\right)$. Since the right-hand side tends to zero, and $P_{\lambda}\left(c_{0}(\lambda)\right)>0$, we obtain $l=0$.

Theorem 5.1.3 and Lemma 5.1.9 yield

Theorem 5.1.10. ${ }^{* 2}$ In the $\mathbf{r}-n o n$-limit-point case there are exactly $n^{-}+\mathbf{r}$ independent solutions of $(2.1)$ in $L_{C_{\lambda}}^{2}$.

In the non-limit-point case, $\mathbf{r}$ equals to the number of eigenvalues of $P_{\lambda}$ that are bounded, by Corollary 5.1.4. Since $P_{\lambda}$ is increasing, we can conclude

Corollary 5.1.11. In the non-limit-point case, equation (2.1) is in the limit-circle case if and only if $P_{\lambda}(x)$ converges as $x$ tends to $b$.

This helps us to show

Lemma 5.1.12. Equation (2.1) is in the full limit-circle case if and only if both (2.1) and (3.15) are in the limit-circle case.

Proof. If $D_{\lambda}(b)$ has interior points, then also $D_{\lambda}(b)-C_{\lambda}(b)$ and $\widehat{D_{\lambda}}(b)-\widehat{C_{\lambda}}(b)=\left(D_{\lambda}(b)-\right.$ $\left.C_{\lambda}(b)\right)^{*}$ have interior points. Hence the ranges of $D_{\lambda}(b)-C_{\lambda}(b)$ and of $\widehat{D_{\lambda}}(b)-\widehat{C_{\lambda}}(b)$ are the whole $\mathbb{C}^{n^{+}}$, respectively the whole $\mathbb{C}^{n^{-}}$.

On the other hand, if (2.1) is in the limit-circle case, then the previous Corollary shows that $P_{\lambda}$ converges to some $P_{\lambda, b}$. If (3.15) is in the limit-circle case, we obtain the same result for $\widehat{P_{\lambda}}$. Therewith (3.47) shows that $\mathcal{R}_{\lambda}$ converges to some $\mathcal{R}_{\lambda}(b)>0$. Since $P_{\lambda}^{-1}$ and $\mathcal{R}_{\lambda}$ are decreasing, any element of

$$
\mathcal{D}:=P_{\lambda, b}^{-1 / 2}\left\{N \in \mathbb{C}^{n^{+}, n^{-}}: N^{*} N \leq I_{n^{-}}\right\} \mathcal{R}_{\lambda}(b)^{1 / 2}
$$

is in $D_{\lambda}(x)-C_{\lambda}(x)$ by (2.27) for every $x \in[a, b)$ and therewith in $D_{\lambda}(b)-C_{\lambda}(b)$ by Theorem 2.2.7.

$\mathcal{D}$ has interior points because $P_{\lambda, b}^{-1 / 2}>\varepsilon I_{n^{+}}$and $\mathcal{R}_{\lambda}(b)^{1 / 2}>\varepsilon I_{n^{-}}$for some $\varepsilon>0$. Thus also $D_{\lambda}(b)$ has interior points. 
It should be mentioned that $\mathbf{r}$ and the analogous value $\widehat{\mathbf{r}}$ for the adjoint case need not coincide. Yet there is an important connection: $\mathbf{r}=\mathbf{0}$ is equivalent to $D_{\lambda}(b)$ consisting of one point. Thus, by Lemma 3.3.1, $\widehat{D_{\lambda}}(b)$ also consists of one point, which means $\widehat{\mathbf{r}}=0$. We obtain

Corollary 5.1.13. (2.1) is in the limit-point case if and only if (3.15) is in the limit-point case.

Furthermore holds

Theorem 5.1.14. (2.1) is in the limit-point case if and only if at least one of the following assertions holds:

(i) There are exactly $n^{-}$independent solutions of $(2.1)$ that are in $L_{C_{\lambda}}^{2}$.

(ii) There are exactly $n^{+}$independent solutions of $(3.15)$ that are in $L_{\widehat{C_{\lambda}}}^{2}$.

Proof. If $\mathcal{R}_{\lambda}(x) \rightarrow 0$ as $x \rightarrow b$, then Lemma 5.1.9 states that (2.1) is in the limit-point case, furthermore (3.47) implies that no eigenvalue of $\widehat{P_{\lambda}}(x)$ is bounded as $x$ tends to $b$. Thus the corresponding assertion of Lemma 5.1.2 for the adjoint case yields that there are exactly $n^{+}$ independent solutions of $(3.15)$ which are in $L_{\overparen{C_{\lambda}}}^{2}$.

If $\mathcal{R}_{\lambda}(x) \nrightarrow 0$, then (3.47) implies that at least one eigenvalue of $\widehat{P_{\lambda}}(x)$ is bounded as $x$ tends to $b$, hence there are more than $n^{+}$independent solutions of (3.15) that are in $L_{\overparen{C_{\lambda}}}^{2}$. So statement $(i i)$ is false.

Theorem 5.1.3 yields: There are exactly $n^{-}$independent solutions of (2.1) that are in $L_{C_{\lambda}}^{2}$ if and only if $\mathbf{r}=0$, which means (2.1) being in the limit-point case.

The statement in Theorem 5.1.14 about the number of linearly independent solutions that lie in $L_{C_{\lambda}}^{2}$ in the limit-point case, is weaker than the corresponding statement in Theorem 5.1 .10 for the non-limit-point case. In section 6.3 below, using an augmentation method, we will obtain a nearly comparable assertion for the limit-point case in Lemma 6.3.3.

Since $C_{\lambda} \succcurlyeq A$, Theorem 5.1.14 yields

Corollary 5.1.15. If (2.1) or (3.15) is in the strict limit-point case, then (2.1) is in the limit-point case.

Furthermore Theorem 5.1.14 and Lemma 4.1.5 imply

Corollary 5.1.16. If $A \asymp C_{\lambda}$, then (2.1) is in the limit-point case if and only if (2.1) or (3.15) is in the strict limit-point case. 
We transfer the proof of [7, Chapter 9, Theorem 2.1] to show

Lemma 5.1.17.*2 If (2.1) and (3.15) are both in the weak limit-circle case, then all solutions of (2.1) are in $L_{A}^{2}$ for all $\lambda \in \mathbb{C}$ and therewith $R_{\lambda}$ is a compact operator.

Proof. Let $\lambda \in \mathbb{C}$ and let $y$ a solution of (2.1).

We choose $\lambda_{0} \in W_{V}$ (so that all columns of $Y_{\lambda_{0}}$ and of $Z_{\lambda_{0}}$ are in $L_{A}^{2}$ ) and $c \in[a, b$ ), such that for

$$
K:=\left(\max \left\{\left|\int_{c}^{b} \xi^{*} Y_{\lambda_{0}}^{*} A Y_{\lambda_{0}} \xi d x\right|,\left\|\int_{c}^{b} Y_{\lambda_{0}}^{*} A Y_{\lambda_{0}} d x\right\|_{\infty},\left\|\int_{c}^{b} J_{0} Z_{\lambda_{0}}^{*} A Z_{\lambda_{0}} J_{0}^{*} d x\right\|_{\infty}\right\}\right)^{1 / 2}
$$

holds $K^{2}<\frac{1}{2 n\left|\lambda-\lambda_{0}\right|}$, where $\|\cdot\|_{\infty}$ means the maximum norm in $\mathbb{C}^{n, n}$, and $\xi \in \mathbb{C}^{n}$ is as in Lemma 3.4.1 for the chosen function $y$.

For any $d \in[c, b), x \in[c, d]$, and $\Psi_{1}, \Psi_{2} \in L_{A}^{2}(c, d)$, we define

$$
\left\langle\psi_{1}, \psi_{2}\right\rangle_{x}:=\int_{c}^{x} \psi_{2}^{*}(s) A(s) \psi_{1}(s) d s
$$

and correspondingly $\|\cdot\|_{x}$.

In the following we use the notation $(\cdot)_{j}$ for the $j^{\text {th }}$ column. The Schwarz inequality in $L_{A}^{2}(c, x)$ yields

$$
\left|\left\langle y,\left(Z_{\lambda_{0}} J_{0}^{*}\right)_{j}\right\rangle_{x}\right| \leq\|y\|_{x}\left\|\left(Z_{\lambda_{0}} J_{0}^{*}\right)_{j}\right\|_{x} \leq K\|y\|_{x} \leq K\|y\|_{d}
$$

for $j \leq n$. Correspondingly we obtain

$$
\left|\left\langle Y_{\lambda_{0}} \xi, y\right\rangle_{d}\right| \leq K|| y \|_{d}
$$

Using (3.51), (5.7) and (5.6), we can estimate 


$$
\begin{aligned}
\|y\|_{d}^{2} & =\left\langle x \mapsto Y_{\lambda_{0}}(x) \xi-\left(\lambda-\lambda_{0}\right) Y_{\lambda_{0}}(x) J_{0} \int_{c}^{x} Z_{\lambda_{0}}^{*}(s) A(s) y(s) d s, y\right\rangle_{d} \\
& \leq K\|y\|_{d}+\left|\lambda-\lambda_{0}\right|\left|\left\langle x \mapsto Y_{\lambda_{0}}(x) J_{0} \int_{c}^{x} Z_{\lambda_{0}}^{*}(s) A(s) y(s) d s, y\right\rangle_{d}\right|^{x} \\
& =K\|y\|_{d}+\left|\lambda-\lambda_{0}\right|\left|\int_{c}^{d}\left(y^{*}(x) A(x) Y_{\lambda_{0}}(x)\right)\left(\int_{c}^{x}\left(Z_{\lambda_{0}} J_{0}^{*}\right)^{*}(s) A(s) y(s) d s\right) d x\right| \\
& =K\|y\|_{d}+\left|\lambda-\lambda_{0}\right|\left|\sum_{j=1}^{n} \int_{c}^{d}\left(y^{*}(x) A(x) Y_{\lambda_{0}}(x)\right)_{j}\left\langle y,\left(Z_{\lambda_{0}} J_{0}^{*}\right)_{j}\right\rangle_{x} d x\right| \\
& \leq K\|y\|_{d}+K|| y \|_{d}\left|\lambda-\lambda_{0}\right| \sum_{j=1}^{n} \int_{c}^{d}\left|y^{*}(x) A(x)\left(Y_{\lambda_{0}}(x)\right)_{j}\right| d x \\
& \leq K\|y\|_{d}+K\|y\|_{d}\left|\lambda-\lambda_{0}\right| \sum_{j=1}^{n}\|y\|_{d}\left\|\left(Y_{\lambda_{0}}\right)_{j}\right\| \|_{d} \\
& \leq K\|y\|_{d}+n K^{2}\left|\lambda-\lambda_{0}\right|\|y\|_{d}^{2} \leq K\|y\|_{d}+\frac{1}{2}\|y\|_{d}^{2} .
\end{aligned}
$$

Thus, $\|y\|_{d} \leq 2 K$ for every $d \in[c, b)$ and hence $y \in L_{A}^{2}[c, b)$. The result follows, since any solution of (2.1) lies in $L_{A}^{2}[a, c]$.

If all solutions of $(2.1)$ are in $L_{A}^{2}$, then $G_{\lambda}$, defined in (4.34), is a Hilbert-Schmidt kernel. Thus $R_{\lambda}$ is compact.

\subsection{Connection between the limit sets and the deficiency index of the maximal operator}

For this section we assume that Assumption 3 holds true, furthermore that for $\lambda \in W_{V}$ only the trivial solution of (2.1) vanishes in $L_{A}^{2}$.

We consider again the deficiency index $d_{\lambda}$ of the maximal operator for (2.1).

Corollary 4.5.4 states $d_{\lambda}=n^{-}+\operatorname{dim}\left[\Phi_{\lambda}\right]_{A}$ for $\lambda \in W_{V}$. Therewith, Lemma 5.1.1 and Theorem 5.1.6 show that $\lambda \mapsto d_{\lambda}$ is constant on $W_{V}$.

Now we assume $A \asymp C_{\lambda}$ (which, for example, holds true in the self-adjoint setting with the canonical choice of $V$ ).

Therewith $L_{A}^{2}$ equals $L_{C_{\lambda}}^{2}$ as a set of classes of functions. Thus, $d_{\lambda}=n^{-}+\operatorname{dim}\left[\Phi_{\lambda}\right]_{C_{\lambda}}$. 
Using Corollary 5.1.4, we obtain that $d_{\lambda}$ is equal to $n^{-}$plus the number of bounded eigenvalues of $P_{\lambda}$ and equal to the number of linearly independent solutions of (2.1), which lie in $L_{C_{\lambda}}^{2}$.

If $\mathcal{R}_{\lambda}(x) \nrightarrow 0$ as $x \rightarrow b$, then it is also equal to $n^{-}+\mathbf{r}$.

If $\mathbf{r}>0$ then (2.1) is in the non-limit-point case, whence $\mathcal{R}_{\lambda}(x) \nrightarrow 0$. Thus we obtain

$$
\mathbf{r}>0 \Longrightarrow d_{\lambda}=n^{-}+\mathbf{r}
$$

The latter equation shows a connection between the geometrical shape of the limit sets and the deficiency index of the maximal operator in the case $A \asymp C_{\lambda}$.

Now we withdraw the assumption $A \asymp C_{\lambda}$.

We still have $C_{\lambda} \succcurlyeq A$, whence any function $\Phi_{\lambda} \xi \in L_{C_{\lambda}}^{2}$ (for some $\xi \in \mathbb{C}^{n^{+}}$) also lies in $L_{A}^{2}$. Hence $d_{\lambda}=n^{-}+\operatorname{dim}\left[\Phi_{\lambda}\right]_{A} \geq n^{-}+\operatorname{dim}\left[\Phi_{\lambda}\right]_{C_{\lambda}}$. As above, we obtain correspondingly

$$
\mathbf{r}>0 \Longrightarrow d_{\lambda} \geq n^{-}+\mathbf{r}
$$

With $n^{-} \leq d_{\lambda} \leq n^{-}+n^{+}$we conclude

$$
n^{-}+\mathbf{r} \leq d_{\lambda} \leq n^{-}+n^{+} .
$$

This again shows a connection between the geometrical shape of the limit sets and the deficiency index of the maximal operator in the general case.

\subsection{Properties of the M-function}

As usual in the Titchmarsh-Weyl theory we have

Theorem 5.3.1. ${ }^{*_{1}} M$ is analytic on $W_{V}$.

Proof. For $\lambda \in W_{V}$, we first want to show the relation

$$
\Psi_{\lambda}=\Psi_{\mu}+(\lambda-\mu) R_{\lambda} \Psi_{\mu} .
$$

Since $\Psi_{\mu}$ solves $J \Psi_{\mu}^{\prime}=(\mu A+B) \Psi_{\mu}$, we obtain

$$
J \Psi_{\mu}^{\prime}=(\lambda A+B) \Psi_{\mu}+(\mu-\lambda) A \Psi_{\mu} .
$$

Equation (4.37) implies

$$
\Psi_{\mu}=(\mu-\lambda) R_{\lambda} \Psi_{\mu}+\Psi_{\lambda} \Xi_{1}+\Phi_{\lambda} \Xi_{2},
$$


for some matrices $\Xi_{1} \in \mathbb{C}^{n^{-}, n^{-}}, \Xi_{2} \in \mathbb{C}^{n^{+}, n^{-}}$, because $\left(\Psi_{\lambda} \mid \Phi_{\lambda}\right)$ is a fundamental matrix for (2.1). We pre-multiply with $\left(I_{n^{-}} \mid 0\right) E^{-1}$ and evaluate (5.12) at $a$. Using $\Psi_{\lambda}=\Theta_{\lambda}+\Phi_{\lambda} M(\lambda)$, furthermore $Y(a)=E$, and (4.36), we obtain $\Xi_{1}=I_{n^{-}}$.

Moreover pre-multiplication with $\zeta_{\lambda}^{*} J$ and use of (4.11) gives

$$
\begin{aligned}
{\left[\Psi_{\mu}, \zeta_{\lambda}\right]-\left[\Psi_{\lambda}, \zeta_{\lambda}\right]-(\mu-\lambda)\left[R_{\lambda} \Psi_{\mu}, \zeta_{\lambda}\right] } & =\left[\Phi_{\lambda}, \zeta_{\lambda}\right] \Xi_{2} \\
& =\left(\left[\Phi_{\lambda}, \eta_{\lambda}\right]+M(\lambda)\left[\Phi_{\lambda}, \chi_{\lambda}\right]\right) \Xi_{2} \\
& =-\Xi_{2} .
\end{aligned}
$$

With (4.11), again, we obtain

$$
\left[\Psi_{\lambda}, \zeta_{\lambda}\right]=\left(I_{n^{-}} \mid M(\lambda)\right)\left[Y_{\lambda}, Z_{\lambda}\right]\left(\begin{array}{c}
I_{n^{-}} \\
M(\lambda)
\end{array}\right)=\left(I_{n^{-}} \mid M(\lambda)\right)\left(\begin{array}{cc}
0 & -I_{n^{+}} \\
I_{n^{-}} & 0
\end{array}\right)\left(\begin{array}{c}
I_{n^{-}} \\
M(\lambda)
\end{array}\right)=0
$$

Using furthermore (4.31) and (4.38), we see that every column of the left-hand-side of (5.13) tends to zero as $x$ tends to $b$. Thus $\Xi_{2}=0$. This proves equation (5.11).

Therewith, using Theorem 4.2.3, we obtain:

$$
\begin{aligned}
M(\lambda) & =M_{0}+(\lambda-\mu) \int_{a}^{b} \zeta_{\mu}^{*} A \Psi_{\lambda} d x \\
& =M_{0}+(\lambda-\mu) \int_{a}^{b} \zeta_{\mu}^{*} A \Psi_{\mu} d x+(\lambda-\mu)^{2}\left\langle R_{\lambda} \Psi_{\mu}, \zeta_{\mu}\right\rangle_{L_{A}^{2}} .
\end{aligned}
$$

By Theorem 4.4.8, $R_{\lambda}$ is the resolvent of $L$ at $\lambda$, thus $\lambda \mapsto R_{\lambda}$ is analytic on $\rho(L) \supset W_{V}$ (cf. [15, III, Theorem 6.7]). Hence (5.15) shows that $\lambda \rightarrow M(\lambda)$ is analytic on $W_{V}$.

We can use the information of the proof of Theorem 5.3.1 to extend the $M$-function analytically on $\rho(L)$ :

Corollary 5.3.2. ${ }^{* 1}$ By (5.15), the definition

$$
M(\lambda):=M_{0}+(\lambda-\mu) \int_{a}^{b} \zeta_{\mu}^{*} A \Psi_{\mu} d x+(\lambda-\mu)^{2}\left\langle(L-\lambda)^{-1} \Psi_{\mu}, \zeta_{\mu}\right\rangle_{L_{A}^{2}}
$$

is an analytic extension of $M$ to $\rho(L)$.

Theorem 5.3.3.* ${ }^{* 3}$ If (2.1) is in the weak limit-circle case, then (5.16) defines a meromorphic extension of $M$ to the whole of $\mathbb{C}$. The poles of $M$ are eigenvalues of $L$.

If also (3.15) is in the weak limit-circle case, then every eigenvalue of $L$ is a pole of $M$.

Proof. In the weak limit-circle case, for $\lambda=\mu$ all solutions of (2.1) are in $L_{A}^{2}$.

Therewith, equations (4.34) and (4.35) imply that $(L-\mu)^{-1}=R_{\mu}$ is a Hilbert-Schmidt operator and thus compact. Hence the spectrum of $L$ consists only of isolated eigenvalues with finite algebraic multiplicity and $(L-\lambda)^{-1}$ is compact for all $\lambda \in \mathbb{C}$ except these eigenvalues. 
Meromorphicity of $\lambda \mapsto(L-\lambda)^{-1}$ with poles only at the eigenvalues of $L$, follows, for example, from [15, III, §5].

Equation (5.16) shows that any pole of $M$ is a pole of $\lambda \mapsto(L-\lambda)^{-1}$, and thus in the spectrum of $L$.

If also (3.15) is in the weak limit-circle case, Lemma 5.1.17 and a corresponding statement for the adjoint problem yield that for every $\lambda \in \mathbb{C}$, all solutions of (2.1) and of (3.15) are in $L_{A}^{2}$. $M$ has no poles in the resolvent set of $L$. Thus, (4.36) defines an extension of $R_{\lambda}$ to $\rho(L)$.

$R_{\lambda}$ is a bounded operator $L_{A}^{2} \rightarrow L_{A}^{2}$. With the same argumentation as for $\lambda \in W_{V}$, we obtain $R_{\lambda}=(L-\lambda)^{-1}$.

Let $\nu \in \mathbb{C}$ such that $M$ has no pole in $\nu$. Thus $M$ is bounded on a neighborhood $N$ of $\nu$. Theorem 3.4.2 (ii) and a corresponding statement for $Z_{\lambda}$ yield that $\lambda \mapsto\left\|\left(Y_{\lambda}\right)_{j}\right\|_{L_{A}^{2}}$ and $\lambda \mapsto\left\|\left(Z_{\lambda}\right)_{j}\right\|_{A}$ are locally bounded for any $j \leq n$. So the same holds for $\lambda \mapsto\left\|\left(\Phi_{\lambda}\right)_{j}\right\|_{A}$, $\lambda \mapsto\left\|\left(\Psi_{\lambda}\right)_{j}\right\|_{A}, \lambda \mapsto\left\|\left(\zeta_{\lambda}\right)_{j}\right\|_{A}$, and $\lambda \mapsto\left\|\left(\chi_{\lambda}\right)_{j}\right\|_{A}$, where $\Psi_{\lambda}=\Theta_{\lambda}+\Phi_{\lambda} M(\lambda)$ and $\zeta_{\lambda}=$ $\eta_{\lambda}+\chi_{\lambda} M(\lambda)^{*}$. We assume w.l.o.g. that these functions are bounded on $N$.

We want to show that $\lambda \mapsto\left\|R_{\lambda}\right\|_{L_{A}^{2} \rightarrow L_{A}^{2}}$ is bounded on $N$. Therefor let $f \in L_{A}^{2}$ with $\|f\|_{A}=1$. We set

$$
g_{\lambda}(x):=\Psi_{\lambda}(x) \int_{a}^{x} \chi_{\lambda}^{*}(y) A(y) f(y) d y
$$

and

$$
h_{\lambda}(x):=\Phi_{\lambda}(x) \int_{x}^{b} \zeta_{\lambda}^{*}(y) A(y) f(y) d y .
$$

Thus we have $R_{\lambda} f=g_{\lambda}+h_{\lambda}$ by (4.34) and (4.35). It suffices to show $\lambda \mapsto\left(\left\|g_{\lambda}\right\|\left\|_{A},\right\| h_{\lambda} \|\left.\right|_{A}\right)$ is bounded on $N$ with an upper bound independent of $f$. We show this assertion for $h_{\lambda}$. The proof for $g_{\lambda}$ is analogous.

$$
\begin{aligned}
& \left\|h_{\lambda}\right\|_{A}^{2}=\int_{a}^{b}\left(\int_{x}^{b} \bar{f}(y) A(y) \zeta_{\lambda}(y) d y \quad \Phi_{\lambda}^{*}(x) A(x) \Phi_{\lambda}(x) \quad \int_{x}^{b} \zeta_{\lambda}^{*}(y) A(y) f(y) d y\right) d x \\
& =\sum_{j, k=1}^{n^{-}} \int_{a}^{b}\left(\left(\int_{x}^{b} \bar{f}(y) A(y) \zeta_{\lambda}(y) d y\right)_{j}\left(\Phi_{\lambda}^{*}(x) A(x) \Phi_{\lambda}(x)\right)_{j, k}\left(\int_{x}^{b} \zeta_{\lambda}^{*}(y) A(y) f(y) d y\right)_{k}\right) d x \\
& =\sum_{j, k=1}^{n^{-}} \int_{a}^{b}\left(\left(\Phi_{\lambda}^{*}(x) A(x) \Phi_{\lambda}(x)\right)_{j, k}\left\langle\left(\zeta_{\lambda}\right)_{j}, f\right\rangle_{L_{A}^{2}(x, b)}\left\langle f,\left(\zeta_{\lambda}\right)_{k}\right\rangle_{L_{A}^{2}(x, b)}\right) d x \\
& \leq \sum_{j, k=1}^{n^{-}} \int_{a}^{b}\left(\left|\left(\Phi_{\lambda}^{*}(x) A(x) \Phi_{\lambda}(x)\right)_{j, k}\right|\left\|\left(\zeta_{\lambda}\right)_{j}\right\|_{L_{A}^{2}(x, b)}\left\|\left(\zeta_{\lambda}\right)_{k}\right\|_{L_{A}^{2}(x, b)}\right) d x \\
& \leq \sum_{j, k=1}^{n^{-}}\left\|\left(\zeta_{\lambda}\right)_{j}\right\|_{A}\left\|\left(\zeta_{\lambda}\right)_{k}\right\|_{A}\left\|\left(\Phi_{\lambda}\right)_{j}\right\|_{A}\left\|\left(\Phi_{\lambda}\right)_{k}\right\|_{A}
\end{aligned}
$$


The righthand side is bounded on $N$ as a function of $\lambda$. Thus we know, that

$$
\lambda \mapsto\left\|R_{\lambda}\right\|_{L_{A}^{2} \rightarrow L_{A}^{2}}=\left\|(L-\lambda)^{-1}\right\|_{L_{A}^{2} \rightarrow L_{A}^{2}}
$$

is bounded on a punctured neighborhood of $\nu$. This shows that $\nu$ is no eigenvalue of $L$.

For the cases $n^{+}=0$ or $n^{-}=0$, Theorem 5.3.1 and Corollary 5.3.2 bear no information, because $M=0_{n^{+}, n^{-}}$has no entries. But Theorem 5.3.3 with the stronger assumption yields $\rho(L)=\mathbb{C}$.

\subsection{Further spectral estimates}

This section consists of two parts.

In the first part $(2.1)$ is examined on $[\tilde{a}, b)$ for some $\tilde{a}>a$. This may yield better spectral estimates (cp. [6, Theorem 4.5]).

The second part assumes the strict limit-point case. By variation of the admissible matrix $V$, we also obtain further spectral estimates (cp. [5, Theorem 6.4]).

\subsubsection{Restriction of the problem to $[\tilde{a}, b) \subseteq[a, b)$}

To consider (2.1) on $[\tilde{a}, b)$, we need one constraint such that Assumption 2 (see page 59) remains true. Therefor we set

$$
\begin{aligned}
& \widehat{b}:=\sup \left\{\tilde{a} \in[a, b): \text { For all } f \in L_{A}^{2} \text { with } \operatorname{supp} f \subset[\tilde{a}, b), \operatorname{ess} \operatorname{supp} A R_{\mu} f \subseteq[a, \tilde{a}]\right. \\
& \text { and }\left[R_{\mu} f, \chi_{\mu}\right](\tilde{a})=0 \text { holds } f=0 \text { in } L_{A}^{2} . \\
& \text { Furthermore for all } g \in L_{A}^{2} \text { with } \operatorname{supp} g \subset[\tilde{a}, b), \operatorname{ess} \operatorname{supp} A \widehat{R}_{\mu} g \subseteq[a, \tilde{a}] \\
&\text { and } \left.\left[\widehat{R}_{\mu} g, \Phi_{\mu}\right](\tilde{a})=0 \text { holds } g=0 \operatorname{in} L_{A}^{2} \cdot\right\},
\end{aligned}
$$

where the essential support of a function $f$ is defined by

$$
\text { ess } \operatorname{supp}(f):=\bigcap\left\{{\overline{\left\{x \in D(f) \backslash N_{0}: f(x) \neq 0\right\}}}^{D(f)}: N_{0} \text { has measure } 0\right\} .
$$

As we will see $\tilde{a}<\widehat{b}$ is necessary and sufficient for Assumption 2 to hold, when restricting the problem to $[\tilde{a}, b)$. But, unless we can choose $\tilde{a} \geq c_{0}(\mu)$, another problem occurs with (2.10), the condition that provides eventually definiteness of $P_{\lambda}$ for all $\lambda$ in $W_{V}$.

Fortunately Corollary 5.4.3 below yields two criteria, which imply $\widehat{b}=b$. Furthermore if $P_{\lambda}(a)>0$ then we could set $c_{0}(\mu)=a$. 
Theorem 5.4.1. Let $\tilde{a} \in[a, \widehat{b})$. If we consider $(2.1)$ on $[\tilde{a}, b)$ and name $\widetilde{W}_{V}=\widetilde{W}_{V}^{\tilde{a}}$ the set which corresponds to $W_{V}$, we have the following result:

(i) $\widetilde{W}_{V} \supseteq W_{V}, \widetilde{W}_{V}=\widetilde{W}_{V}^{\tilde{a}}$ is increasing as a function of $\tilde{a}$.

(ii) If $\widehat{b}>c_{0}(\mu)$, then $\widetilde{W}_{V} \backslash \rho(L)$ consists at most of isolated points.

(iii) If furthermore, for $\lambda \in \widetilde{W}_{V} \backslash \rho(L)$, only the trivial solution of (2.1) vanishes in $L_{A}^{2}$, then $\lambda$ lies in $\sigma_{p}(L)$, the point spectrum of $L$.

In [6, Theorem 4.7] a corresponding result is shown in the limit-point case for second order Sturm-Liouville problems.

Proof. For the proof we also name all further corresponding quantities for the problem on $[\tilde{a}, b)$ with a tilde.

If we recall $W_{V}=\operatorname{Adm}_{V}+\Lambda_{V}$, Definitions (2.5) and (2.6) yield statement $(i)$.

Now we assume $\widehat{b}>c_{0}(\mu)$.

With $(i)$, we can furthermore assume w.l.o.g. $\tilde{a}>c_{0}(\mu)$ and that $\tilde{a}$ fulfills the premise in the braces of (5.17).

If $A=0$ a.e. on $[\tilde{a}, b)$, then both, (2.1) and (3.15) are in the weak limit-circle case, because any column of $Y_{\lambda}$ or of $Z_{\lambda}$ is continuous and $A \in L_{\text {loc }}^{1}[a, b)$. Lemma 5.1.17 yields that the whole spectrum of $L$ consists of isolated points in $\sigma_{p}(L)$.

So we can furthermore assume w.l.o.g. $A_{\mid[\tilde{a}, b]} \neq 0$ on a set of positive measure. This is the only fundamental requirement, which may be violated by considering the problem on $[\tilde{a}, b)$.

Let $\widetilde{E}:=Y_{\mu}(\tilde{a})$. We first show that $(V, \widetilde{E})$ is suitable for problem $(2.1)$ on $[\tilde{a}, b)$ (with $\left.\widetilde{n^{+}}=n^{+}, \tilde{n^{-}}=n^{-}\right)$.

Admissibility of $V$ is equivalent to $\widetilde{W}_{V}$ not being empty, which is given by $(i)$. Using the definition of $\widetilde{E}$, equation (2.8) yields

$$
\widetilde{P}_{V, \widetilde{E}}(\tilde{a})=\frac{1}{2} \Phi_{\mu}^{*}(\tilde{a}) V \Phi_{\mu}(\tilde{a})=P(\tilde{a})>0,
$$

since $\tilde{a}>c_{0}(\mu)$. Thus Remark 2.1.2 shows suitability of $(V, \widetilde{E})$.

Again by $(i)$ we know $\mu \in \widetilde{W}_{V}$. Since $Y_{\mu}$ solves $(2.1)$ and $Y_{\mu}(\tilde{a})=\widetilde{E}$, we obtain $\widetilde{Y_{\mu}}=\left.Y_{\mu}\right|_{[\tilde{a}, b)}$. Therewith, the corresponding relations hold for $\widetilde{\Theta_{\mu}}, \widetilde{\Phi_{\mu}}, \widetilde{Z_{\mu}}, \widetilde{\eta_{\mu}}$, and $\widetilde{\chi_{\mu}}$ by $(2.7),(3.20),(3.19)$ and (4.34). 
Using (2.11), we can conclude $\widetilde{D_{\mu}}(c)=D_{\mu}(c)$ for every $c \in[\tilde{a}, b)$. So by definition $\widetilde{D_{\mu}}(b)=D_{\mu}(b)$ and thus $M_{0} \in \widetilde{D_{\mu}}(b)$. Hence we can choose the same pair $\left(\mu, M_{0}\right)$ for the construction of the $M$-function $\widetilde{M}$. In particular, we obtain $\widetilde{M}(\mu)=M_{0}=M(\mu)$. With the above knowledge we obtain $\widetilde{\Psi_{\mu}}=\left.\Psi_{\mu}\right|_{[\tilde{a}, b)}, \widetilde{\zeta_{\mu}}=\left.\zeta_{\mu}\right|_{[\tilde{a}, b)}$, and $\widetilde{G_{\lambda}}=\left.G_{\lambda}\right|_{[\tilde{a}, b)}$.

We still need to show that Assumption 2 is fulfilled for the problem on $[\tilde{a}, b)$.

Therefor let $\tilde{f} \in L_{A}^{2}[\tilde{a}, b)$ such that $\tilde{y}:=\widetilde{R_{\mu}} \tilde{f}$ is zero in $L_{A}^{2}(\tilde{a}, b)$. We have to show $\tilde{f}=0$ in $L_{A}^{2}[\tilde{a}, b)$.

For the function

$$
f:[a, b) \rightarrow \mathbb{C}^{n}, f:=\left\{\begin{array}{l}
0 \text { on }(a, \tilde{a}) \\
\tilde{f} \text { on }[\tilde{a}, b)
\end{array}\right.
$$

we know that $\widetilde{R_{\mu}} \tilde{f}=R_{\mu} f_{\mid[\tilde{a}, b)}$ by (4.35). With (4.38) we obtain that $f$ fulfills the corresponding premise in the braces of (5.17). Thus, by assumption on $\tilde{a}$, we conclude $f=0$ in $L_{A}^{2}$ and hence $\tilde{f}=0$ in $L_{A}^{2}[\tilde{a}, b)$. A corresponding conclusion shows that $\widehat{R}_{\mu}$ is one-to-one.

(As we can see, the existence of such an $f \neq 0$ or $g \neq 0$ in $L_{A}^{2}(\tilde{a}, b)$, which fulfills the corresponding premise in the braces of (5.17), would violate Assumption 2 for the problem on $[\tilde{a}, b)$.)

All necessary assumptions are fulfilled for the problem, restricted on $[\tilde{a}, b)$. Hence all results of the previous chapters hold correspondingly true. The theory in chapter 4 yields an operator $\widetilde{L}$ with $\widetilde{W}_{V} \subseteq \rho(\widetilde{L})$.

We pick an arbitrary $\lambda \in \widetilde{W}_{V}$, and want to examine whether $\lambda$ is in the resolvent set of $L$ or not. We recall that the property $\lambda \in \rho(L)$ means that, for any $f \in L_{A}^{2}$, there exists a unique $g \in D(L)$ such that $(L-\lambda) g=f$ and the mapping $f \mapsto g$ is bounded.

So let $f \in L_{A}^{2}$.

In the following, for functions $\xi:[a, b) \rightarrow \mathbb{C}^{m_{1}, m_{2}}$ (with $m_{1}, m_{2} \in \mathbb{N}_{0}$ ), we use the notations

$$
\xi^{\vdash}:=\left.\xi\right|_{[\tilde{a}, b)}, \quad \xi^{\dashv}:=\left.\xi\right|_{[a, \tilde{a}]} .
$$

For $g_{2}:=\widetilde{R}_{\lambda} f^{\vdash}$ holds $g_{2} \in D(\widetilde{L})$ and $\widetilde{L} g_{2}=\lambda f^{\vdash}$, which means

$$
\begin{aligned}
& g_{2} \in L_{A}^{2}(\tilde{a}, b) \cap A C_{\mathrm{loc}}[\tilde{a}, b), \\
& {\left[g_{2}, \zeta_{\mu}\right](b)=\left[g_{2}, \widetilde{\zeta}_{\mu}\right](b)=0,} \\
& J g_{2}^{\prime}=(\lambda A+B) g_{2}+A f^{\vdash},
\end{aligned}
$$


and a boundary condition at $\tilde{a}$, which we are not interested in. For any $\xi_{2} \in \mathbb{C}^{n^{-}}$the function

$$
g_{2}+\widetilde{\Psi_{\lambda}} \xi_{2}
$$

has the same properties by (4.30), and for regularity reasons, every function with these properties, has the form (5.21).

For $x \in[a, \tilde{a}]$, we define $g_{1}(x):=\int_{a}^{\tilde{a}} G_{\lambda}(x, y) A(y) f(y) d y$.

Since $A \in L_{\text {loc }}^{1}$, the same calculations as for Corollary 4.3.2 and for (4.38) yield

$$
\begin{gathered}
g_{1} \in A C_{\mathrm{loc}}[a, \tilde{a}] \cap L_{A}^{2}(a, \tilde{a}), \\
{\left[g_{1}, \chi_{\mu}\right](a)=\left[g_{1}, \chi_{\lambda}\right](a)=0,} \\
J g_{1}^{\prime}=(\lambda A+B) g_{1}+A f^{\dashv} .
\end{gathered}
$$

Using (4.11), we obtain that for any $\xi_{1} \in \mathbb{C}^{n^{+}}$the function

$$
g_{1}+\Phi_{\lambda}^{\dashv} \xi_{1}
$$

has the same properties, and every function with these properties, has the form (5.25). lent:

By definition of $D(L)$, for any function $g:[a, b) \rightarrow \mathbb{C}^{n}$ the following assertions are equiva-

(i)

$$
g \in D(L) \text { and }(L-\lambda) g=f \text {. }
$$

(ii) $g^{\vdash}$ fulfills properties (5.18) to (5.20) and $g^{\dashv}$ fulfills properties (5.22) to (5.24).

So any solution of (5.26) has the form $g^{\dashv}=g_{1}+\Phi_{\lambda}^{\dashv} \xi_{1}, g^{\vdash}=g_{2}+\widetilde{\Psi_{\lambda}} \xi_{2}$ for some $\xi_{1} \in \mathbb{C}^{n^{+}}$, $\xi_{2} \in \mathbb{C}^{n^{-}}$. And vice versa any such function solves (5.26). The crucial point is continuity in $\tilde{a}$. We conclude:

There is exactly one function $g:[a, b) \rightarrow \mathbb{C}^{n}$ with $g \in D(L)$ and $(L-\lambda) g=f$ if and only if there exists exactly one $\xi=\left(\begin{array}{c}\xi_{1} \\ \xi_{2}\end{array}\right) \in \mathbb{C}^{n}$ such that

$$
\left(g_{1}+\Phi_{\lambda}^{\dashv} \xi_{1}\right)(\tilde{a})=\left(g_{2}+\widetilde{\Psi_{\lambda}} \xi_{2}\right)(\tilde{a}) .
$$

We define

$$
\Omega_{\lambda}:=\left(\Phi_{\lambda}(\tilde{a}) \mid-\widetilde{\Psi_{\lambda}}(\tilde{a})\right)=\left(\Phi_{\lambda}(\tilde{a}) \mid-\widetilde{E}\left(\begin{array}{c}
I_{n^{-}} \\
\widetilde{M}(\lambda)
\end{array}\right)\right)
$$


and $K:=-g_{1}(\tilde{a})+g_{2}(\tilde{a})$.

Therewith (5.27) is equivalent to $\Omega_{\lambda} \xi=K$, which is uniquely solvable if and only if $\Omega_{\lambda}$ is regular.

The knowledge $\rho(L) \subseteq W_{V}$ does not yield regularity of $\Omega_{\lambda}$ for $\lambda \in W_{V}$, it only implies uniqueness of a solution of (5.26) in $L_{A}^{2}$. But for $\lambda=\mu$, the above choice of $\widetilde{E}$ and $\widetilde{M}$ yields

$$
\Omega_{\mu}=\left(\Phi_{\mu}(\tilde{a}) \mid-\Psi_{\mu}(\tilde{a})\right)=Y_{\mu}(\tilde{a})\left(\begin{array}{cc}
0 & -I_{n^{-}} \\
I_{n^{+}} & -M(\mu)
\end{array}\right)
$$

where the right-hand side is a product of regular matrices. Theorems 5.3.1 and $3.4 .2(i)$ show that all entries of the right-hand side of (5.28) are analytic in $\lambda$ and thus this holds for $\Omega_{\lambda}$ and therewith also for its determinant. This is not zero for $\lambda=\mu$.

The set $\widetilde{W}_{V}$, where $\Omega$ is defined, is open and convex by Lemma 5.1.5, so $\lambda \mapsto \operatorname{det}\left(\Omega_{\lambda}\right)$ has only isolated zeros in $\widetilde{W}_{V}$.

Now we choose some $\lambda \in \widetilde{W}_{V}$ with $\operatorname{det}\left(\Omega_{\lambda}\right) \neq 0$.

We have already established that for every $f \in L_{A}^{2}$ there exists exactly one $g \in D(L)$ with $(L-\lambda) g=f$. Finally we have to show boundedness of $(L-\lambda)^{-1}$.

The restrictions $f \mapsto f^{\vdash}$ and $f \mapsto f^{\dashv}$ are bounded $L_{A}^{2} \rightarrow L_{A}^{2}(\tilde{a}, b)$ and $L_{A}^{2} \rightarrow L_{A}^{2}(a, \tilde{a})$, respectively.

Since $\lambda \in \rho(\widetilde{L})$, the mapping $f^{\vdash} \mapsto g_{2}=(\widetilde{L}-\lambda)^{-1} f^{\vdash}$ is bounded, too. This also holds for $f^{\dashv} \mapsto g_{1}$, because every column of $Y_{\lambda}$ and $Z_{\lambda}$ is in $L_{A}^{2}(a, \tilde{a})$.

The calculation

$$
\begin{aligned}
K=-g_{1}(\tilde{a})+g_{2}(\tilde{a}) & =-\int_{a}^{\tilde{a}} G_{\lambda}(\tilde{a}, y) A(y) f(y) d y+\int_{\tilde{a}}^{b} \widetilde{G}_{\lambda}(\tilde{a}, y) A(y) f(y) d y \\
& =-\Psi_{\lambda}(\tilde{a}) \int_{a}^{\tilde{a}} \chi_{\lambda}^{*}(y) A(y) f(y) d y+\widetilde{\Phi_{\lambda}}(\tilde{a}) \int_{\tilde{a}}^{b} \widetilde{\zeta}_{\lambda}^{*}(y) A(y) f(y) d y,
\end{aligned}
$$

shows that $f \mapsto K$ is bounded $L_{A}^{2} \rightarrow \mathbb{C}^{n}$, since $\chi_{\lambda}^{*} \in L_{A}^{2}(a, \tilde{a})$ and $\widetilde{\zeta_{\lambda}} \in L_{A}^{2}(\tilde{a}, b)$. Hence $f \mapsto \xi=\Omega_{\lambda}^{-1} K$ is bounded.

The canonical embeddings $L_{A}^{2}(a, \tilde{a}) \hookrightarrow L_{A}^{2}$ and $L_{A}^{2}(\tilde{a}, b) \hookrightarrow L_{A}^{2}$ are bounded.

Since $\Phi_{\lambda} \in L_{A}^{2}(a, \tilde{a})$ and $\widetilde{\Psi_{\lambda}} \in L_{A}^{2}(\tilde{a}, b)$, we can finally conclude

$$
f \mapsto(L-\lambda)^{-1} f= \begin{cases}g_{1}+\Phi_{\lambda} \xi_{1} & \text { on }[a, \tilde{a}], \\ g_{2}+\widetilde{\Psi_{\lambda}} \xi_{2} & \text { on }[\tilde{a}, b)\end{cases}
$$


is bounded $L_{A}^{2} \rightarrow L_{A}^{2}$. Thus we have $\lambda \in \rho(L)$, which holds true for all $\lambda \in \widetilde{W}_{V}$ with $\operatorname{det}\left(\Omega_{\lambda}\right) \neq 0$.

It remains to show assertion (iii) of this Theorem. So let $\lambda \in \widetilde{W}_{V} \backslash \rho(L)$. We assume that only the trivial solution of (2.1) is in the class of the null-functions of $L_{A}^{2}$. We have to show that $\lambda$ is in the point spectrum of $L$.

We know that $\Omega_{\lambda}$ is singular (otherwise $\lambda \in \rho(L)$ ). Hence there exists some nontrivial $\xi=\left(\begin{array}{l}\xi_{1} \\ \xi_{2}\end{array}\right) \in \mathbb{C}^{n}$ such that

$$
0=\Omega_{\lambda} \xi=\Phi_{\lambda}(\tilde{a}) \xi_{1}-\widetilde{\Psi_{\lambda}}(\tilde{a}) \xi_{2}
$$

This implies continuity of

$$
y:[a, b) \rightarrow \mathbb{C}^{n}, \quad y:= \begin{cases}\Phi_{\lambda} \xi_{1} & \text { on }[a, \tilde{a}], \\ \widetilde{\Psi_{\lambda}} \xi_{2} & \text { on }[\tilde{a}, b),\end{cases}
$$

which is a non-trivial solution of (2.1). Moreover $y \in D(L)$ with $L y=\lambda y$ by (4.52), (4.11), (4.30), and $\widetilde{\zeta}_{\mu}=\zeta_{\mu}^{\vdash}$. By assumption, $y$ is not zero in $L_{A}^{2}$, hence $\lambda$ is in the point spectrum of $L$.

Since $\tilde{a}$ was chosen arbitrarily in $[a, \widehat{b})$, Theorem 5.4.1 implies

Corollary 5.4.2. If $\widehat{b}>c_{0}(\mu)$ then

$$
\bigcup_{\tilde{a} \in[a, \widehat{b})} \widetilde{W}_{V}^{\tilde{a}} \backslash \rho(L) \text { consists at most of isolated points, }
$$

where $\widetilde{W_{V}^{\tilde{a}}}$ means $\widetilde{W}_{V}$ as in Theorem 5.4 .1 with respective choice of $\tilde{a}$.

It should be remarked that those isolated points may accumulate on the boundary of $\bigcup \widetilde{W}_{V}^{\tilde{a}}$.

The proof of Theorem 5.4.1 shows that $\tilde{a}$ fulfills the premise in the braces of (5.17) if and only if Assumption 2 holds for the restricted problem with the suitable pair $\left(V, Y_{\mu}(a)\right)$. Remarks 4.4.1 and 4.4.2 provide criteria which yield Assumption 2. If one of these criteria holds for some $\tilde{a}_{0} \in[a, b)$, then it holds for every $\tilde{a} \in\left[\tilde{a}_{0}, b\right)$. Hence we can conclude

Corollary 5.4.3. If eventually the premises of Remark 4.4.2 or the premises of Remark 4.4 .1 (together with the corresponding premises for $\widehat{R_{\mu}}$ ) are fulfilled, then $\widehat{b}=b$.

Proof. Let $\tilde{a} \in[a, b)$. If $A_{\mid[\tilde{a}, b)}>0$ on a set of positive measure, then the thoughts above show that $\tilde{a}$ fulfills the premise in the braces of (5.17). Otherwise any function $f \in L_{A}^{2}$ with $\operatorname{supp}(f) \in[\tilde{a}, b)$ is zero in $L_{A}^{2}$, whence $\tilde{a}$ fulfills the premise in the braces of (5.17) anyway. 
A second criterion to estimate $\widehat{b}$ from below is the following

Corollary 5.4.4. If $A>0$ on a set of positive measure, then

$$
\widehat{b} \geq \sup \left\{\tilde{a}_{0} \in[a, b): A_{\mid\left[\tilde{a}_{0}, b\right)}(x)>0 \text { on a set of positive measure }\right\} \text {. }
$$

Proof. Let $\tilde{a}_{0} \in[a, b)$ such that $A_{\mid\left[\tilde{a}_{0}, b\right)}(x)>0$ on a set of positive measure. We can choose some $\tilde{a} \in\left[\tilde{a}_{0}, b\right)$ such that for any continuous functions $f$ holds

$$
A f=0 \text { almost everywhere on }[\tilde{a}, b) \Longrightarrow f(\tilde{a})=0 .
$$

We show that $\tilde{a}$ fulfills the premises in the braces of (5.17) for corresponding functions $f$. The statement for corresponding functions $g$ can be shown analogously. Thus, $\widehat{b} \geq \tilde{a} \geq \tilde{a}_{0}$.

So let $f \in L_{A}^{2}$ with supp $f \subseteq[\tilde{a}, b)$ and $\operatorname{ess} \operatorname{supp}\left(A R_{\lambda} f\right) \subseteq[a, \tilde{a}]$. Then, by assumption on $\tilde{a}$, we obtain $R_{\lambda} f(\tilde{a})=0$. The function $y:=\left(R_{\lambda} f\right)_{\mid[a, \tilde{a}]}$ solves

$$
J y^{\prime}=(\lambda A+B) y+f=(\lambda A+B) y
$$

on $[a, \tilde{a}]$ and $y(\tilde{a})=0$. By regularity of this equation we conclude $y=\left(R_{\lambda} f\right)_{\mid[a, \tilde{a}]}=0$. Thus $R_{\lambda} f=0$ in $L_{A}^{2}$, hence $f=0$ in $L_{A}^{2}$.

\subsubsection{Examples}

We take a look at the examples on pages 23 to 30 .

In the case that $A$ has compact essential support, both (2.1) and (3.15) are in the weak limit-circle case, thus we already know that $L$ has only point spectrum. Theorem 5.4.1 does not yield any better spectral estimation. Therefore, in the following consideration of the examples, we assume that, for every $\tilde{a} \in[a, b), A_{\mid[\tilde{a}, b)} \neq 0$ on a set of positive measure.

In the self-adjoint case with the canonical choice of $V$, we know that $W_{V}$ is the upper, respectively the lower halfplane. This does not change when restricting the problem to $[\tilde{a}, b)$. Hence Theorem 5.4.1 yields no further information.

Now we consider the scalar examples, i.e. those examples that model the scalar equations

$$
\tau_{e}[v]:=p_{0} v+\sum_{k=1}^{m}(-1)^{k}\left(\left(p_{k} v^{(k)}\right)^{(k)}-\left(q_{k} v^{(k)}\right)^{(k-1)}-\left(r_{k} v^{(k-1)}\right)^{(k)}\right)=\lambda w v,
$$

respectively

$$
\begin{aligned}
\tau_{o}[v]: & =p_{0} v+(-1)^{m} i\left(s\left(s v^{(m)}\right)^{\prime}\right)^{(m)}+\sum_{k=1}^{m}(-1)^{k}\left(\left(p_{k} v^{(k)}\right)^{(k)}-\left(q_{k} v^{(k)}\right)^{(k-1)}-\left(r_{k} v^{(k-1)}\right)^{(k)}\right) \\
& =\lambda w v .
\end{aligned}
$$


With the additional assumption $w>0$ an $[a, b)$, we have shown that Assumption 2 holds by showing the premises of Remark 4.4.1. Thus Corollary 5.4.3 yields $\widehat{b}=b$. Hence Theorem 5.4.1, respectively Corollary 5.4 .2 can be applied with the result that there are only isolated points of the spectrum of $L$ within

$$
\bigcup_{\tilde{a} \in[a, b)} \widetilde{W}_{V}^{\tilde{a}}
$$

Using the assumption $w>0$ an $[a, b)$, on page 71 we have shown that, for every $\lambda \in \mathbb{C}$, only the trivial solution of $(2.1)$ vanishes in $L_{A}^{2}$.

By Theorem 5.4 .1 (iii), the isolated points of the spectrum of $L$ within $\bigcup_{\tilde{a} \in[a, b)} \widetilde{W}_{V}^{\tilde{a}}$ are in the point spectrum of $L$.

In all the scalar examples we have $A=\operatorname{diag}(w, 0, \ldots, 0)$ and therewith $\Lambda_{V}=\{\lambda \in \mathbb{C}$ : $\operatorname{Re}(\lambda \nu)>0\}$ for some constant $\nu \in \mathbb{C} \backslash\{0\}$ (which is the first entry of $-V J$; for the calculations see page 66). Restricting the problem to $[\tilde{a}, b)$ does not affect $\Lambda_{V}$. Thus, we only have to consider $\operatorname{Adm}_{V}$.

We estimate, for $\tilde{a} \in[a, b)$, the set $\widetilde{\operatorname{Adm}_{V}}$ in the three dimensional case with real leading coefficient function $s$. On page 29, we have calculated that $\operatorname{Adm}_{V}$ is the set of all $\lambda \in \mathbb{C}$ such that

$$
4 v^{2} \mathbf{R e}\left(-i \lambda w+i p_{0}\right) \mathbf{R e}\left(i p_{1}\right)-\left|v\left(q_{1}+\bar{r}_{1}\right)-i v_{0}\right|^{2} \geq 0
$$

almost everywhere on $[a, b)$ (where $v, v_{0}$ are entries of $V$, furthermore $p_{1}, q_{1}, r_{1}$, and $p_{0}$ are given functions of the scalar problem).

The same calculation on the restricted problem yields that $\widetilde{\operatorname{Adm}_{V}}$ is the set of all $\lambda \in \mathbb{C}$ such that (5.33) holds almost everywhere on $[\tilde{a}, b)$. Thus $\widehat{\operatorname{Adm}_{V}}$ may be a proper superset of $\operatorname{Adm}_{V}$.

Correspondingly, in item 3 on page 30, which is the even order example with $m=1$, we obtain that $\widehat{\mathrm{Adm}_{V}}$ is the set of all $\lambda \in \mathbb{C}$ such that the estimations in equation (2.49) hold on $[\tilde{a}, b)$.

\subsubsection{Variation of the admissible matrix $V$}

For the second part of this section we assume that (2.1) or (3.15) is in the strict limit-point case.

For another suitable pair $(\widetilde{V}, \widetilde{E})$ with $n^{+}(\widetilde{V})=n^{+}$and $W_{\widetilde{V}} \cap W_{V} \neq \emptyset$ we have the following results: According to Corollary 5.1.8 (and a corresponding statement for the adjoint problem), (2.1), respectively (3.15), remain in the strict limit-point case. The strict limit-point 
case of (2.1) or of (3.15) implies the limit-point case and thus there is no freedom in choosing an appropriate M-function for such a pair. Let us call it $\widetilde{M}$.

In the following lines, the other corresponding quantities for $(\widetilde{V}, \widetilde{E})$ are also labeled by a tilde.

Recall

$$
\Psi_{\lambda}=Y_{\lambda}\left(\begin{array}{c}
1_{n^{-}} \\
M(\lambda)
\end{array}\right), \quad \zeta_{\lambda}=Z_{\lambda}\left(\begin{array}{c}
1_{n^{+}} \\
M(\lambda)^{*}
\end{array}\right)
$$

which shows that $\Psi_{\lambda}$ and $\zeta_{\lambda}$ have full rank. Using (4.7) or (4.8), respectively, we see that there holds at least one of the statements (depending on whether (2.1) or (3.15) is in the strict limit-point case)

$$
\begin{aligned}
& \text { (i) } \widetilde{\Psi_{\lambda}}=\Psi_{\lambda} \Xi_{1}, \\
& \text { (ii) } \widetilde{\zeta_{\lambda}}=\zeta_{\lambda} \Xi_{2},
\end{aligned}
$$

for some regular matrices $\Xi_{1} \in \mathbb{C}^{n^{-}, n^{-}}$or $\Xi_{2} \in \mathbb{C}^{n^{+}, n^{+}}$, respectively.

If we furthermore assume $\widetilde{E}=E$ (which gives $\widetilde{Y}=Y, \widetilde{Z}=Z$ ), then (5.34) together with any of both statements in (5.35) imply

$$
\widetilde{M}=M \quad \text { on } \quad W_{V} \cap W_{\widetilde{V}}
$$

Let $\mathcal{W}_{V}$ the connected component of

$$
\bigcup_{\substack{(\widetilde{V}, E) \text { suitable } \\ n^{+}(\widetilde{V})=n^{+}}} W_{\widetilde{V}},
$$

which contains $W_{V}$. Since all $W_{\widetilde{V}}$ are open, with the above arguments we obtain, for all suitable pairs $\left(\widetilde{V}_{1}, E\right),\left(\widetilde{V}_{2}, E\right)$ with $n^{+}\left(\widetilde{V}_{1}\right)=n^{+}\left(\widetilde{V}_{2}\right)=n^{+}$and $W_{\widetilde{V}_{1}} \cap W_{\widetilde{V}_{2}} \cap \mathcal{W}_{V} \neq \emptyset$, that $\widetilde{M}_{\widetilde{V}_{1}}(\lambda)=\widetilde{M}_{\widetilde{V}_{2}}(\lambda)$ for $\lambda \in W_{\widetilde{V}_{1}} \cap W_{\widetilde{V}_{2}}$. Thus Theorem 5.3.1 yields

Corollary 5.4.5.*3 If (2.1) or (3.15) is in the strict limit-point case, then the definition

$$
M(\lambda):=\widetilde{M}(\lambda) \quad \text { for }(\widetilde{V}, E) \text { suitable with } n^{+}(\widetilde{V})=n^{+} \text {and } \lambda \in W_{\widetilde{V}},
$$

is a well-defined analytic extension of $M$ to $\mathcal{W}_{V}$.

Furthermore we can conclude

Theorem 5.4.6. If (2.1) or (3.15) is in the strict limit-point case, then $\mathcal{W}_{V}$ is a subset of $\rho(L)$. 
Proof. Let $\widetilde{V}$ admissible with $(\widetilde{V}, E)$ suitable, $n^{+}(\widetilde{V})=n^{+}$, and $W_{V} \cap W_{\widetilde{V}} \neq \emptyset$.

We choose $\widetilde{\mu} \in W_{V} \cap W_{\widetilde{V}}$ and $\widetilde{M}_{0} \in \widetilde{D}_{b}(\widetilde{\mu})$ to construct an $M$-function for $(\widetilde{V}, E)$ and therewith $\widetilde{R}_{\widetilde{\mu}}$. The considerations above show that there is no other choice but $\widetilde{M}_{0}=M(\widetilde{\mu})$. Since $\widetilde{Y}=Y$ and $\widetilde{Z}=Z$, equations (4.34) and (4.35) imply $\widetilde{R}_{\widetilde{\mu}}=R_{\widetilde{\mu}}$, in particular $\widetilde{R}_{\widetilde{\mu}}$ : $L_{A}^{2} \rightarrow L_{A}^{2}$ is one-to-one. The same holds for $\widetilde{\widehat{R}}_{\widetilde{\mu}}$, so Assumption 2 is fulfilled.

We obtain an operator $\widetilde{L}$, while Theorem 4.4 .8 yields $\widetilde{L}=L$.

The same Theorem implies $W_{\widetilde{V}} \subseteq \rho(\widetilde{L})=\rho(L)$.

Because all $W_{\widetilde{V}}$ are open, standard arguments yield the statement on $\mathcal{W}_{V}$.

We can use this knowledge and connect it with the theory in the first part of this section.

For the case that (2.1) or (3.15) is in the strict limit-point case, the proof of Theorem 5.4.6 even shows the following: For any suitable pair $(\widetilde{V}, E)$ with $n^{+}(V)=n^{+}$and $W_{\widetilde{V}} \subseteq \mathcal{W}_{V}$, Assumption 2 is fulfilled and the resulting differential operator $\widetilde{L}$ equals $L$.

Now we show that the quantity $\widetilde{b}$, that corresponds to $\widehat{b}$ for the suitable pair $(\widetilde{V}, E)$ with some choice of $\widetilde{\mu} \in W_{\widetilde{V}}$ equals $\widehat{b}$ : We have established that $\tilde{a} \in[a, b)$ fulfills the properties in the braces of (5.17) if and only if Assumption 2 holds true for problem (2.1) restricted to $[\tilde{a}, b)$ with the suitable pair $\left(V, Y_{\mu}(\tilde{a})\right)$. By definition, $\widehat{b}$ is the supremum of all these $\tilde{a}$.

The strict limit-point case of (2.1), respectively of (3.15), can not get lost by restricting the problem to $[\tilde{a}, b$ ) (for a detailed explanation of this statement see the proof of Theorem 5.4 .8 below). Furthermore $\widetilde{\mathcal{W}}_{V} \supseteq \mathcal{W}_{V}$ and $\widetilde{W_{\widetilde{V}}} \supseteq W_{\widetilde{V}}$, whence $\widetilde{\mathcal{W}}_{V} \cap \widetilde{W_{\widetilde{V}}} \neq \emptyset$ (and thus $\left.\widetilde{W}_{\widetilde{V}} \subseteq \widetilde{\mathcal{W}}_{V}\right)$. The same considerations as above yield that, if Assumption 2 holds true for the restricted problem with the suitable pair $\left(V, Y_{\mu}(\tilde{a})\right)$, then Assumption 2 holds true for the restricted problem with the suitable pair $\left(\widetilde{V}, Y_{\mu}(\tilde{a})\right)$. Thus $\widetilde{b} \geq \widehat{b}$, and by symmetry $\widetilde{\widehat{b}}=\widehat{b}$. If, furthermore, for every such suitable pair, we could assure $\widetilde{c}_{0}(\widetilde{\mu})<\widehat{b}$ (for some choice of $\widetilde{\mu} \in W_{\widetilde{V}}$ ), then Corollary 5.4.2 would yield an estimation of the spectrum of $L$ for the suitable pair $(\widetilde{V}, E)$. The estimation $c_{0}(\widetilde{\mu})<\widehat{b}$ surely holds true if $\widehat{b}=b$ or if $P_{\lambda}(a)>0$ (whence we can choose $\widetilde{c}_{0} \equiv a$ for all $\left.\widetilde{V}\right)$. We conclude

Corollary 5.4.7. Let

$$
\widehat{\mathcal{W}_{V}}:=\bigcup_{\widetilde{V} \in \mathcal{T}} \bigcup_{\tilde{a} \in[a, \widehat{b})} \widetilde{W}_{\widetilde{V}}^{\tilde{a}},
$$

where $\mathcal{T}$ consist of all admissible $\widetilde{V}$, such that $(\widetilde{V}, E)$ is suitable, $n^{+}(V)=n^{+}$and $W_{\widetilde{V}} \subseteq \mathcal{W}_{V}$. If $\left(\widehat{b}=b\right.$ or $\left.P_{\lambda}(a)>0\right)$ and at least one of the equations (2.1) or (3.15) is in the strict limit-point case, then $\widehat{\mathcal{W}_{V}} \backslash \rho(L)$ consist at most of isolated points.

In the following, we use the notation $\mathbb{S}^{n \times}$ for the set of all self-adjoint, regular matrices. 
We can go one step further:

Theorem 5.4.8. For $\tilde{a} \in[a, b)$ let $\mathcal{P}_{V}^{\tilde{a}}$ the connected component of

$$
\bigcup\left\{\widetilde{W_{\widetilde{V}}^{\tilde{a}}}: \widetilde{V} \in \mathbb{S}^{n \times}, n^{+}(\widetilde{V})=n^{+}, \text {and }\left(0 I_{n^{+}}\right) Y_{\mu}^{*}(\tilde{a}) \widetilde{V} Y_{\mu}(\tilde{a})\left(\begin{array}{c}
0 \\
I_{n^{+}}
\end{array}\right)>0\right\},
$$

which contains $\widetilde{W_{V}^{\tilde{a}}}$.

If $\widehat{b}>c_{0}(\mu)$ and at least one of the equations (2.1) or (3.15) is in the strict limit-point case, then

$$
\bigcup_{\tilde{a} \in\left[c_{0}(\mu), \widehat{b}\right)} \mathcal{P}_{V}^{\tilde{a}} \backslash \rho(L)
$$

consist at most of isolated points.

Proof. Let $\tilde{a} \in[a, \widehat{b})$. The proof is mostly analog to the proof of Theorem 5.4.1. With the same arguments we can assume w.l.o.g. that $A_{\mid[\tilde{a}, b)} \neq 0$ on a set of positive measure.

We need to show that $\mathcal{P}_{V}^{\tilde{a}}$ is increasing as a function of $\tilde{a}$.

As in the proof of Theorem 5.4.1, from its definition we see that $W_{\tilde{V}}^{\tilde{a}}$ is increasing as a function of $\tilde{a}$. It suffices to show that

$$
\widetilde{V} \in \mathbb{S}^{n \times}, \quad \widetilde{W}_{\tilde{V}}^{\tilde{a}} \neq \emptyset, \quad \text { and } \quad\left(0 I_{n^{+}}\right) Y_{\mu}^{*}(\tilde{a}) \widetilde{V} Y_{\mu}(\tilde{a})\left(\begin{array}{c}
0 \\
I_{n^{+}}
\end{array}\right)>0
$$

implies $\left(0 I_{n^{+}}\right) Y_{\mu}^{*}(\widetilde{d}) \widetilde{V} Y_{\mu}(\widetilde{d})\left(\begin{array}{c}0 \\ I_{n^{+}}\end{array}\right)>0$ for $\tilde{d}>\tilde{a}$

(5.38) implies that $\left(\widetilde{V}, Y_{\mu}(\tilde{a})\right)$ is suitable for the problem restricted on $[\tilde{a}, b)$, with $\widetilde{P}_{\mu}(\tilde{a})>0$, whence $\widetilde{P}_{\mu}$ is increasing by Lemma 2.2.4. Thus we obtain

$$
\left(0 I_{n^{+}}\right) Y_{\mu}^{*}(\widetilde{d}) \widetilde{V} Y_{\mu}(\widetilde{d})\left(\begin{array}{c}
0 \\
I_{n^{+}}
\end{array}\right)=2 \widetilde{P}_{\mu}(\widetilde{d})>0
$$

So $\mathcal{P}_{V}^{\tilde{a}}$ is increasing as a function of $\tilde{a}$. Therewith we can assume w.l.o.g. that $\tilde{a}$ fulfills the premise in the braces of (5.17) (which is necessary for Assumption 2 to hold).

With the suitable pair $\left(V, Y_{\mu}(\tilde{a})\right)$, as in the proof of Theorem 5.4.1, we obtain an operator $\widetilde{L}$ with resolvent set $\rho(\widetilde{L})$. In this proof we have used $\widetilde{W}_{V}^{\tilde{a}} \subseteq \rho(\widetilde{L})$, to show that in the set $\widetilde{W}_{V}^{\tilde{a}}$ only isolated points of the spectrum of $L$ may exist. The same proof even shows that in the open set $\rho(\widetilde{L})$ only isolated points of the spectrum of $L$ may exist. (Yet for obvious reasons, this statement could not be part of the statement of the Theorem.)

Thus it suffices to show $\widetilde{W_{\widetilde{V}}^{\tilde{a}}} \subseteq \rho(\widetilde{L})$ for any self-adjoint matrix $\widetilde{V}$ with $n^{+}(V)=n^{+}, \widetilde{W}_{\widetilde{V}}^{\tilde{a}} \subseteq \mathcal{P}_{V}^{\tilde{a}}$, and which fulfills (5.38). (For $W_{\widetilde{V}}^{\tilde{a}}=\emptyset$, the statement would be trivial.) 
Again, (5.38) implies that $\left(\widetilde{V}, Y_{\mu}(\tilde{a})\right)$ is suitable for the restricted problem.

All columns of $Z_{\lambda}$ and $Y_{\lambda}$ are continuous, $A \in L_{\text {loc }}^{1}$, thus all columns of $Z_{\lambda}$ and $Y_{\lambda}$ are in $L_{A}^{2}(a, \tilde{a})$. Hence the number of linearly independent solution of (2.1), respectively of (3.15), which are in $L_{A}^{2}$, equals the corresponding number for the restricted problem. Thus, by assumption and $n^{-}(\widetilde{V})=n^{-}$, at least one of the corresponding equations, $\widetilde{(2.1)}$ or $\widetilde{(3.15)}$, is in the strict limit-point case.

Theorem 5.4.6, applied to the restricted problem, yields $\widetilde{\mathcal{W}_{V}} \subseteq \rho(\widetilde{L})$. By definition of $\mathcal{P}_{V}^{a}$ and of $\mathcal{W}_{V}$ (on page 95), we obtain

$$
\widetilde{W_{\tilde{V}}^{\tilde{a}}} \subseteq \mathcal{P}_{V}^{\tilde{a}} \subseteq \widetilde{\mathcal{W}_{V}} \subseteq \rho(\widetilde{L})
$$

\subsubsection{Examples}

We like to apply Theorem 5.4.6, Corollary 5.4.7, and Theorem 5.4.8 to example 3 on page 30. This means

$$
J=J_{1}=\left(\begin{array}{ll} 
& -1 \\
1 &
\end{array}\right), \quad A=A_{1}=\left(\begin{array}{cc}
w & \\
& 0
\end{array}\right), \quad B=B_{1}=\frac{1}{p}\left(\begin{array}{cc}
-q r-p p_{0} & -q \\
r & 1
\end{array}\right) .
$$

Recall that for $Y=\left(y_{0}, y_{1}\right)^{T}$, equation $(2.1)$ is equivalent to $y_{1}=y_{0}^{[1]}$ and

$$
p_{0} y_{0}-\left(p y_{0}^{\prime}\right)^{\prime}-q y_{0}^{\prime}-\left(r y_{0}\right)^{\prime}=\lambda w y_{0} .
$$

We already know that any admissible matrix has the form

$$
V=\left(\begin{array}{cc}
v_{0} & \bar{v} \\
v & 0
\end{array}\right)
$$

where $v_{0}$ is real and $v \neq 0$. Such a matrix $V$ is not definite, whence $n^{+}=n^{-}=1$. Thus we will require that $\Phi_{\lambda}$ or $\chi_{\lambda}$ is not in $L_{A}^{2}$ to obtain the strict limit-point case for (2.1) or for (3.15), respectively (which is necessary to apply the desired Theorems).

The first entry of $-V J$ is $-\bar{v}$. We conclude that (see (4.58))

$$
\Lambda_{V}=\{\lambda \in \mathbb{C}: \operatorname{Re}(-\lambda \bar{v})>0\}
$$

1. We first examine the case that $B$ is self-adjoint (thus $p, p_{0}$ are real and $q=-\bar{r}$ ). 
We fix the initial matrix $E=E_{c}=\left(\begin{array}{cc}1 & -i \\ -i & 1\end{array}\right)$

For the canonical choice $V_{c}=i J_{1}=\left(\begin{array}{cc}0 & -i \\ i & 0\end{array}\right)$, we already know that $\Lambda_{V_{c}}$ is the lower halfplane, so is $W_{V_{c}}$. This is a subset of the resolvent set of the corresponding operator $L_{c}$.

We want to construct further admissible matrices $\widetilde{V}$ with rotated sets $W_{\widetilde{V}}$, for which $(\widetilde{V}, E)$ is suitable. We choose $\operatorname{Re} v=1$. Because $B$ is self-adjoint, equation (2.49), which is equivalent to $\lambda \in \operatorname{Adm}_{\tilde{V}}$, reduces to

$$
v_{0} \mathbf{R e} r+q r+p p_{0}-\boldsymbol{R e}(\lambda \bar{v}) p w-\frac{1}{4} v_{0}^{2} \succcurlyeq 0 .
$$

We assume $p w \succcurlyeq-q r-p p_{0}$ with a corresponding (preferably small) $l_{0} \in \mathbb{R}$ such that $l_{0} p w+q r+p p_{0} \succcurlyeq 0$. (If $q r+p p_{0} \succcurlyeq 0$, even $l_{0} \leq 0$ is possible.)

The choice $v_{0, k}:=0, v_{k}:=1+k i$ provides the matrix $V_{k}=\left(\begin{array}{cc}0 & 1-k i \\ 1+k i & 0\end{array}\right)$.

$\left(V_{k}, E_{c}\right)$ is suitable for $k>0$ by straightforward calculation and Remark 2.1.2, with

$$
\begin{gathered}
\lambda_{k}:=-\frac{l_{0}}{\left(1+k^{2}\right)}(1+k i) \in \operatorname{Adm}_{V_{k}}, \\
\Lambda_{V_{k}}=\left\{\lambda \in \mathbb{C}: \operatorname{Re}\left(-\lambda \bar{v}_{k}\right) \geq 0\right\}=\{\lambda \in \mathbb{C}:-k \operatorname{Im} \lambda-\operatorname{Re} \lambda>0\} .
\end{gathered}
$$

We conclude

$$
\lambda_{k}+\Lambda_{V_{k}} \subseteq W_{V_{k}} .
$$

By Theorem 5.4.6 the union of all these sets for $k>0$ is in the resolvent set of $L_{c}$.

Our estimation of the spectrum of $L_{c}$ would be even better if we could also choose $v$ with negative imaginary part. Therefor we additionally assume

$$
p w \succcurlyeq-\boldsymbol{R e}(r) \quad \text { and } \quad p w \succcurlyeq 1 \text {. }
$$

Let $l_{1}, l_{2} \in \mathbb{R}$ such that $l_{1} p w+\mathbf{R e}(r) \succcurlyeq 0$ and $l_{2} p w-1 \succcurlyeq 0$. (Again $l_{1}$ could be non-positive.)

For $k<0$ and $\varepsilon>0$ we obtain an admissible matrix $V_{k}^{\varepsilon}$ by choosing $v_{0, k, \varepsilon}:=\varepsilon-2 k$, $v_{k, \varepsilon}:=1+k i$.

$\left(V_{k}^{\varepsilon}, E_{c}\right)$ is suitable by Remark 2.1.2 with

$$
\begin{gathered}
\lambda_{k}^{\varepsilon}:=-\left(l_{0}+v_{0, k, \varepsilon} l_{1}+\frac{1}{4} v_{0, k, \varepsilon}^{2} l_{2}\right)\left(1+k^{2}\right)^{-1} v_{k, \varepsilon} \in \operatorname{Adm}_{V_{k}^{\varepsilon}}, \\
\Lambda_{V_{k}^{\varepsilon}}=\left\{\lambda \in \mathbb{C}: \operatorname{Re}\left(-\lambda \bar{v}_{k, \varepsilon}\right) \geq 0\right\}=\{\lambda \in \mathbb{C}:-k \operatorname{Im} \lambda-\operatorname{Re} \lambda>0\} .
\end{gathered}
$$


We conclude

$$
\lambda_{k}^{\varepsilon}+\Lambda_{V_{k}^{\varepsilon}} \subseteq W_{V_{k}^{\varepsilon}} \subseteq \rho(L) .
$$

This holds for every $\varepsilon>0$. We set

$$
\lambda_{k}:=\lambda_{k}^{0}=-\frac{\left(l_{0}-2 k l_{1}+k^{2} l_{2}\right)}{\left(1+k^{2}\right)}(1+k i) .
$$

By standard arguments we conclude that also the open set

$$
\lambda_{k}+\{\lambda \in \mathbb{C}:-k \operatorname{Im} \lambda-\operatorname{Re} \lambda>0\}
$$

is a subset of the resolvent set of $L$ for $k<0$, and therewith the union of these.

Now we assume that $\Phi_{\lambda}$ or $\chi_{\lambda}$ is not in $L_{A}^{2}$, so we have the strict limit-point case for (2.1) or for $(3.15)$.

If, by restriction of the functions on $[\tilde{a}, b)$ for some $\tilde{a} \in[a, b)$, the constants $l_{0}^{\tilde{a}}, l_{1}^{\tilde{a}}$, or $l_{2}^{\tilde{a}}$ could be chosen smaller, then the corresponding sets $\lambda_{k}^{\tilde{a}}+\Lambda_{V_{k}}$ are larger. Furthermore these are subsets of $\widehat{\mathcal{W}_{V}}$. By Corollary 5.4.7, in the union of these sets are only isolated points of the spectrum of $L_{c}$.

2. We consider the case $q=r=0$. So we are in the setting of [6] and of [5, example on page 425$]$.

Thus $B=\operatorname{diag}\left(-p_{0}, \frac{1}{p}\right)$. Again we have to consider matrices $V$ of the form (5.39).

With $q=r=0$, in view of (2.49), any choice of $V$ with $v_{0} \neq 0$ would not yield any larger set $\operatorname{Adm}_{V}$ than with $v_{0}=0$. Hence we only consider matrices of the form $V=\operatorname{codiag}(v, \bar{v})$. Multiplication of $V$ with a positive real factor does not change the result, so it suffices to consider the matrices

$$
V=V_{\varphi}:=\operatorname{codiag}\left(e^{i \varphi}, e^{-i \varphi}\right)
$$

for $\varphi \in[0,2 \pi)$. Therewith, equation (2.49) reduces to

$$
\boldsymbol{\operatorname { R e }}\left(e^{-i \varphi}\left(p_{0}-\lambda w\right)\right) \succcurlyeq 0 \text { and } \boldsymbol{\operatorname { R e }}\left(e^{-i \varphi} p\right) \succcurlyeq 0 .
$$

$\operatorname{Adm}_{V_{\varphi}}$ is the set of all $\lambda$, for which (5.42) holds. In [5] the existence of some $\lambda$ with the same properties is required.

By $(5.40), \Lambda_{V_{\varphi}}$ is the following halfplane

$$
\Lambda_{V_{\varphi}}=\{\lambda \in \mathbb{C}: \operatorname{Re}(-\lambda \bar{v}) \geq 0\}=\left\{\lambda \in \mathbb{C}: \operatorname{Re}\left(\lambda e^{-i \varphi}\right)<0\right\} .
$$


2.1 We want to consider the following example in [6, page 1251]:

$[a, b)=[1, \infty), \quad \arg (p)=\alpha$ on $[1, \infty), \quad p_{0}(x)=c_{1} x^{d_{1}}+i c_{2} x^{d_{2}}, \quad w(x)=x^{\omega}$,

for real constants $\alpha, c_{1}, c_{2}, d_{1}, d_{2}$, and $\omega$ with $\alpha \in\left[-\frac{\pi}{2}, \frac{\pi}{2}\right], c_{1}<0, c_{2} \leq 0, \quad d_{2}>d_{1}>\omega$. We exclude the cases $\alpha \in\left\{-\frac{\pi}{2}, \frac{\pi}{2}\right\}$ or $c_{2}=0$ (for simplicity and to assure the existence of an admissible matrix.)

The second condition in (5.42) reads $\varphi \in\left[\alpha-\frac{\pi}{2}, \alpha+\frac{\pi}{2}\right]$, (modulo $2 \pi$ ).

The first condition in $(5.42)$ reads

$$
\left(x \mapsto \boldsymbol{R e}\left(e^{-i \varphi}\left(c_{1} x^{d_{1}}+i c_{2} x^{d_{2}}-\lambda x^{\omega}\right)\right) \succcurlyeq 0,\right.
$$

which is equivalent to

$$
\boldsymbol{R e}\left(-\lambda e^{-i \varphi}\right) \geq x^{d_{1}-\omega}\left(-c_{1} \cos \varphi-c_{2} x^{d_{2}-d_{1}} \sin \varphi\right) \quad \text { for all } x \geq 1 .
$$

The right-hand side tends, as $x \rightarrow \infty$, to plus infinity for $\varphi \in[0, \pi)$ and to minus infinity for $\varphi \in(-\pi, 0)$, while the lefthand side is constant. Thus $\operatorname{Adm}_{V_{\varphi}}=\emptyset$ for $\varphi \in[0, \pi)$, but for $\varphi \in(-\pi, 0)$, equation (5.43) shows that $\operatorname{Adm}_{V_{\varphi}}$ is a halfplane.

We conclude that $V_{\varphi}$ is admissible exactly for $\varphi \in\left[\alpha-\frac{\pi}{2}, 0\right)$.

With $E:=\left(\begin{array}{ll}1 & 1 \\ 0 & i\end{array}\right)$, we obtain $P_{\lambda, \varphi}(1)>0$, for $\varphi \in(-\pi, 0)$, whence $\left(E, V_{\varphi}\right)$ is suitable for $\varphi \in\left[\alpha-\frac{\pi}{2}, 0\right)$.

We fix some $\varphi_{o} \in\left(\alpha-\frac{\pi}{2}, 0\right)$. For the resulting differential operator $L_{\varphi_{o}}$ we know $\mathrm{Adm}_{V_{\varphi_{o}}}+$ $\Lambda_{V_{\varphi_{o}}} \subseteq \rho\left(L_{\varphi_{o}}\right)$.

With $w>0$ on $[a, b)=[1, \infty)$, we have shown for all our scalar examples that $\widehat{b}=b$. We like to apply Corollary 5.4.2. For $\tilde{a} \in[1, \infty)$, we have $\widetilde{W}_{V_{\varphi_{0}}}^{\tilde{a}}=\widetilde{\operatorname{Adm}}_{V_{\varphi_{o}}}^{\tilde{a}}+\widetilde{\Lambda}_{V_{\varphi_{o}}}^{\tilde{a}}$, where $\widetilde{\Lambda}_{V_{\varphi_{0}}}^{\tilde{a}}=\Lambda_{V_{\varphi}}$. Hence we have to calculate $\bigcup_{\tilde{a} \in[1, \infty)} \widetilde{\operatorname{Adm}_{V_{\varphi_{0}}}^{\tilde{a}}}$.

For $\tilde{a} \in[1, \infty)$, by the first condition of $(5.43)$, we obtain that $\lambda \in \widetilde{\operatorname{Adm}}_{V_{\varphi_{0}}}^{\tilde{a}}$ if and only if

$$
\boldsymbol{R e}\left(-\lambda e^{-i \varphi}\right) \geq x^{d_{1}-\omega}\left(-c_{1} \cos \varphi_{o}-c_{2} x^{d_{2}-d_{1}} \sin \varphi_{o}\right) \quad \text { for all } x \geq \tilde{a} .
$$

Since the right-hand side tends to minus infinity, as $x \rightarrow \infty$, for every $\lambda \in \mathbb{C}$, there exists an $\tilde{a} \in[1, \infty)$ such that $\lambda \in \widetilde{\operatorname{Adm}_{V_{\varphi_{0}}}^{\tilde{a}}}$. Hence $\bigcup_{\tilde{a} \in[1, \infty)} \widetilde{\operatorname{Adm}}_{V_{\varphi_{0}}}^{\tilde{a}_{0}}=\mathbb{C}$.

Corollary 5.4.2 yields that the spectrum of $L_{\varphi_{o}}$ consists of isolated points.

Now we assume that $\Phi_{\lambda}$ or $\chi_{\lambda}$ is not in $L_{A}^{2}$, so we have the strict limit-point case for $(2.1)$ or for $(3.15)$. 
Thus, Theorem 5.4.6 yields

$$
\bigcup_{\varphi \in\left[\alpha-\frac{\pi}{2}, 0\right)}\left(\operatorname{Adm}_{V_{\varphi}}+\Lambda_{V_{\varphi}}\right) \subset \rho\left(L_{\varphi_{o}}\right) .
$$

Note, that $\Lambda_{V_{\varphi}}$ is a halfplane with rotation $\varphi$ relative to the left halfplane.

In [6], a corresponding differential operator is constructed. Its spectrum is estimated, in general, to be in a (possibly shifted and rotated) halfplane.

In the case that only one solution is square integrable (this is the strict limit-point case in this thesis), the spectrum is estimated to be a subset of some set $Q(a)$, which may be "smaller" than a halfplane. For this particular example, the estimation of $\mathbb{C} \backslash Q(a)$ are alike the left-hand side of (5.45).

2.2 We consider the following example to give an application for Theorem 5.4.8:

$$
[a, b)=[1, \infty), w \succcurlyeq\left|p_{0}(\cdot)\right|, \quad p(x)=-x^{d_{1}}-i c x^{d_{2}}, w(x)>0 \quad(\text { for } x \in[1, \infty)),
$$

for real constants $c, d_{1}$, and $d_{2}$, with $c>0$ and $d_{2}>d_{1}$.

Let $\beta(x):=\arg (p(x))=\pi+\arctan \left(c x^{d_{2}-d_{1}}\right)$. We have $\beta(x) \in\left(\pi, \frac{3}{2} \pi\right)$, and $\beta$ converges monotonically to $\frac{3}{2} \pi$, thus range $(\beta)=\left[\beta(1), \frac{3}{2} \pi\right)$. The second condition in (5.42) becomes

$$
\forall x \geq 1: \operatorname{Re}\left(e^{i(\beta(x)-\varphi)}\right) \geq 0,
$$

which is equivalent to $\varphi \in\left[\pi, \beta(1)+\frac{\pi}{2}\right]$ (modulo $\left.2 \pi\right)$.

The first condition in (5.42) becomes

$$
\boldsymbol{\operatorname { R e }}\left(-\lambda e^{-i \varphi}\right) w \geq \boldsymbol{R e}\left(-p_{0} e^{-i \varphi}\right),
$$

on $[1, \infty)$. With $w \succcurlyeq\left|p_{0}\right|$, we see that $(5.47)$ is solvable for every $\varphi$.

We conclude that $V_{\varphi}=\operatorname{codiag}\left(e^{i \varphi}, e^{-i \varphi}\right)$ is admissible exactly for $\varphi \in\left[\pi, \beta(1)+\frac{\pi}{2}\right]$. (5.47) shows that $\operatorname{Adm}_{V_{\varphi}}$ is a halfplane.

With $E:=\left(\begin{array}{ll}1 & i \\ 0 & 1\end{array}\right)$, we obtain $P_{\lambda, V_{\varphi}, E}(1)>0$, for $\varphi \in(\pi, 2 \pi)$, whence $\left(E, V_{\varphi}\right)$ is suitable for $\varphi \in\left(\pi, \beta(1)+\frac{\pi}{2}\right]$.

For fixed $\varphi_{o} \in\left(\pi, \beta(1)+\frac{\pi}{2}\right]$ we obtain an operator $L_{\varphi_{o}}$ with $\operatorname{Adm}_{V_{\varphi_{o}}}+\Lambda_{V_{\varphi_{o}}} \subseteq \rho\left(L_{\varphi_{o}}\right)$.

We want to apply Theorem 5.4.8. Therefor we assume, that $\Phi_{\lambda}$ or $\chi_{\lambda}$ is not in $L_{A}^{2}$, so we have the strict limit-point case for (2.1) or for (3.15). (If this was not the case, both 
problems would be in the weak limit-circle case, whence the whole spectrum of of $L_{\varphi_{o}}$ would consist of isolated eigenvalues.)

Again we know that $\widehat{b}=\infty$.

Theorem 5.4.8 yields that in $\widetilde{W}_{V_{\varphi}}^{\tilde{a}}$ only isolated points of the spectrum of $L_{\varphi_{o}}$ exist for all $\tilde{a} \in\left[c_{0}(\mu), \infty\right)$, and for all matrices $V_{\varphi}$ that are suitable for the problem restricted to $[\tilde{a}, b)$ such that $\widetilde{W}_{V_{\varphi}}^{\tilde{a}} \cap W_{V_{\varphi_{0}}} \neq \emptyset$ and for which holds

$$
\left(0 I_{n^{+}}\right) Y_{\mu}^{*}(\tilde{a}) V_{\varphi} Y_{\mu}(\tilde{a})\left(\begin{array}{c}
0 \\
I_{n^{+}}
\end{array}\right)>0 .
$$

(This matrix is $2 \widetilde{P}_{\mu, V_{\varphi}, Y_{\mu}(\tilde{a})}(\tilde{a})$ for the problem restricted to $[\tilde{a}, b)$ ).

First we calculate the set of matrices $V_{\varphi}$ that are admissible for the problem on $[\tilde{a}, b)$. Equation (5.46) becomes

$$
\forall x \geq \tilde{a}: \operatorname{Re}\left(e^{i(\beta(x)-\varphi)}\right) \geq 0,
$$

which is equivalent to $\varphi \in\left[\pi, \beta(\tilde{a})+\frac{\pi}{2}\right]$ (modulo $2 \pi$ ). If we recall $\beta(\tilde{a}) \rightarrow \frac{3}{2} \pi$, as $\tilde{a} \rightarrow \infty$, we see that, for every $\varphi \in\left[\pi, \frac{3}{2} \pi\right)$, there exists an $\tilde{a}>1$ such that (5.49) holds true.

For such $\varphi$, the set $\widetilde{\operatorname{Adm}}_{V_{\varphi}}^{\tilde{a}}$ is the set of all $\lambda$ that fulfill $(5.47)$ on $[\tilde{a}, \infty)$. As above we see that this is a closed halfplane. Thus $\widetilde{W}_{V_{\varphi}}^{\tilde{a}}$ is a halfplane by Lemma 5.1.5 .

Unfortunately not every admissible matrix is suitable for the restricted problem with $\widetilde{E}=$ $Y_{\mu}(\tilde{a})$. (We even require that the matrix corresponding to $P(a)$ is definite.)

For $\varphi_{1}:=\beta(1)+\frac{\pi}{2}$, though, we know $P_{\mu, V_{\varphi_{1}}, Y_{\mu}(1)}(1)>0$ and that $\left(V_{\varphi_{1}}, E\right)$ is admissible for the original problem, whence $P_{\mu, V_{\varphi_{1}}, Y_{\mu}(1)}$ is increasing. Thus, for $\tilde{a}>1$,

$$
0<2 P_{\mu, V_{\varphi_{1}}, Y_{\mu}(1)}(\tilde{a})=\left(\begin{array}{ll}
0 & I_{n^{+}}
\end{array}\right) Y_{\mu}^{*}(\tilde{a}) V_{\varphi_{1}} Y_{\mu}(\tilde{a})\left(\begin{array}{c}
0 \\
I_{n^{+}}
\end{array}\right) .
$$

By continuity reasons, we obtain

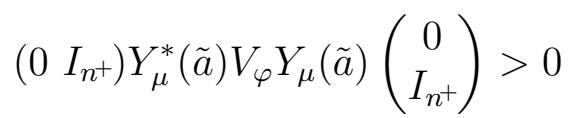

for all $\varphi$ in a neighborhood of $\varphi_{1}$. So the set of all matrices $V_{\varphi}$ that are suitable for the restricted problem and that fulfill (5.48), is larger than the set of all suitable matrices for the original problem. Therewith, for $\tilde{a}>1$, the set

$$
\bigcup\left\{\widetilde{W}_{V_{\varphi}}^{\tilde{a}}: V_{\varphi} \in \mathbb{S}^{n \times}, n^{+}\left(V_{\varphi}\right)=n^{+}, \widetilde{W}_{V_{\varphi}}^{\tilde{a}} \cap W_{V_{\varphi_{o}}} \neq \emptyset, \text { and }\left(0 I_{n^{+}}\right) Y_{\mu}^{*}(\tilde{a}) V_{\varphi} Y_{\mu}(\tilde{a})\left(\begin{array}{c}
0 \\
I_{n^{+}}
\end{array}\right)>0\right\}
$$

is larger than the same set with $\tilde{a}=1$. (Note that, again, the parameter $\varphi$ determines the rotation of the halfplane $\widetilde{W_{V_{\varphi}}^{\tilde{a}}}$.) There are only isolated points of the spectrum of $L_{\varphi_{o}}$ in the union of all these sets for $\tilde{a} \in[1, \infty)$. 


\section{Chapter 6}

\section{Final considerations}

In this chapter, with some linear algebra theory, we obtain an insight into various possibilities of choosing parameters (like V).

An augmentation method, which augments the problem to an equivalent problem of higher order, yields further miscellaneous results.

Finally we examine the one dimensional example (i.e. $n=n^{+}+n^{-}=1$ ).

Below we use the notation $\mathbb{S}^{n}$ for the set of all self-adjoint matrices in $\mathbb{C}^{n, n}$ (and again $\mathbb{S}^{n \times}$ shall name the set of all regular matrices in $\mathbb{S}^{n}$ ).

\subsection{On the choice of an admissible $V$}

Assumption 1 requires the existence of an admissible matrix $V$. The number of positive (respectively negative) eigenvalues of $V$ determine the sizes of nearly every matrix in this theory. If there are two admissible matrices $V_{1}$ and $V_{2}$ with $n^{+}\left(V_{2}\right) \neq n^{+}\left(V_{1}\right)$, we obtain two operators, for which the number of boundary conditions at $a$ (and maybe at $b$ ) are different. So it is a natural question, if, for given $A, B$, and $J$, such admissible matrices $V_{1}$ and $V_{2}$ may exist.

We will realize that, under certain conditions, for such matrices the sets $\mathrm{Adm}_{V_{1}}$ and $\mathrm{Adm}_{V_{1}}$ are disjoint or this may hold for $\Lambda_{V_{1}}$ and $\Lambda_{V_{2}}$.

We first need some linear algebraic results.

Lemma 6.1.1. If $K \in \mathbb{C}^{n, n}$ is singular, then $\operatorname{Re} K$ is not definite.

Proof. Let $0 \neq b \in \mathbb{C}^{n}$ with $K b=0$. Then $2 b^{*}(\boldsymbol{R e} K) b=b^{*} K b+b^{*} K^{*} b=0$. 
Lemma 6.1.2. Let $K \in \mathbb{C}^{n, n}$. For

$$
\mathcal{K}:=\left\{N \in \mathbb{S}^{n}: \operatorname{Re}(N K)>0\right\}
$$

holds

(i) $\mathcal{K}$ is an open wedge within $\mathbb{S}^{n}$, in particular $\mathcal{K}$ is convex.

(ii) For $N_{1}, N_{2} \in \mathcal{K}$ holds $n^{+}\left(N_{1}\right)=n^{+}\left(N_{2}\right)$.

Proof. $\mathcal{K}$ is open since both $\mathbf{R e}$ and multiplication with $K$, are continuous.

We note that, for $t>0$ and $N \in \mathcal{K}$, also $t N \in \mathcal{K}$. For $N_{1}, N_{2} \in \mathcal{K}$, by linearity we obtain $N_{1}+N_{2} \in \mathcal{K}$. Thus assertion $(i)$ follows.

For $j \in\{0, \ldots, n\}$ we define $\mathcal{M}_{j}:=\left\{N \in \mathbb{S}^{n \times}: n^{+}(N)=j\right\}$. These sets are obviously disjoint and open in $\mathbb{S}^{n}$. By Lemma 6.1 .1 we know that $\mathcal{K}$ is a subset of the union of these sets. Since $\mathcal{K}$ is connected within $\mathbb{S}^{n}$, it is a subset of $\mathcal{M}_{j}$ for some $j \leq n$. Thus assertion (ii) follows.

Lemma (6.1.2) with $K=\lambda J A$, and (2.6) yield

Corollary 6.1.3. If $A$ is positive definite on a set of positive measure, then for any admissible matrices $V_{1}, V_{2}$ for which there exists $\lambda \in \Lambda_{V_{1}} \cap \Lambda_{V_{2}}$ holds $n^{+}\left(V_{1}\right)=n^{+}\left(V_{2}\right)$.

We even know

Lemma 6.1.4. If the matrix $A$ is positive definite on a set of positive measure then for any admissible $V$ holds

$$
n^{+}(V) \in\left\{n^{+}( \pm i J)\right\}
$$

while $n^{+}(V)=n^{+}(i J)$ holds if there is some $\lambda \in \Lambda_{V}$ with $\operatorname{Im}(\lambda)<0$ and $n^{+}(V)=n^{+}(-i J)$ holds if there is some $\lambda \in \Lambda_{V}$ with $\operatorname{Im}(\lambda)>0$.

Proof. By Theorem 6.2.2 below any $\lambda \in \Lambda_{V}$ is non-real.

Let $\lambda$ in the lower half-plane. For $V_{1}:=i J$,

$$
-\boldsymbol{R e}\left(\lambda V_{1} J A\right)=-\boldsymbol{R e}(\lambda(i J) J A)=\operatorname{Re}((i \lambda) A)=-(\operatorname{Im}(\lambda)) A \succcurlyeq A
$$

shows $\lambda \in \Lambda_{V_{1}}$. Corollary 6.1.3 implies the assertion for any admissible $V$ with $\lambda \in \Lambda_{V}$.

The assertion for $\lambda$ in the upper half-plane can be shown analogously with $V_{2}:=-i J$. 
We need one more linear algebraic

Lemma 6.1.5. Let $K \in \mathbb{C}^{n, n}$. We define

$$
\begin{aligned}
\mathcal{K} & :=\left\{N \in \mathbb{S}^{n \times}: \operatorname{Re}(N K)>0\right\}, \\
\mathcal{K}_{0} & :=\left\{N \in \mathbb{S}^{n}: \operatorname{Re}(N K) \geq 0\right\} .
\end{aligned}
$$

(i) Either $\mathcal{K}$ is empty or $\mathcal{K}_{0}$ is the closure of $\mathcal{K}$ in $\mathbb{S}^{n}$.

(ii) If $\mathcal{K}$ is not empty, then for any regular matrices $N_{1}, N_{2} \in \mathcal{K}_{0}$ holds $n^{+}\left(N_{1}\right)=n^{+}\left(N_{2}\right)$.

Proof. Since $0 \in \mathcal{K}_{0}$, at least one of both assertions in $(i)$ is wrong. Thus it suffices to show: if $\mathcal{K}$ is not empty, then it is dense in $\mathcal{K}_{0}$, which is obviously closed in $\mathbb{S}^{n}$ by standard continuity arguments.

Now let $N_{0} \in \mathcal{K}_{0}$ and $\delta>0$. If $\mathcal{K}$ is not empty, since it is an open wedge by Lemma 6.1.2, there exists some $N \in \mathcal{K}$ with $\|N\|\|K\|<\delta$ (where $\|\cdot\|$ is the operator norm $\mathbb{C}^{n} \rightarrow \mathbb{C}^{n}$ ). By linearity $\operatorname{Re}\left(\left(N+N_{0}\right) K\right)>0$ and thus $N+N_{0} \in \mathcal{K}$. Hence statement $(i)$ follows.

For $N_{1}, N_{2} \in \mathcal{K}$, statement (ii) is shown in Lemma 6.1.2. The statement still holds for $N_{1}$, $N_{2} \in \overline{\mathcal{K}}=\mathcal{K}_{0}$ as long as no eigenvalue is zero, by continuity of the eigenvalues with respect to every matrix-norm.

Regarding assumptions (2.3) and (2.4) for admissibility, we obtain

Lemma 6.1.6. Let

$\widetilde{A d m}:=\left\{k \in \mathbb{C}: \exists U \in \mathbb{S}^{n \times}:-\boldsymbol{R e}(U J(k A+B))>0\right.$ on more than a set of measure zero $\}$.

For admissible $V_{1}, V_{2}$ with $A d m_{V_{1}} \cap A d m_{V_{2}} \cap \widetilde{A d m} \neq \emptyset$ holds $n^{+}\left(V_{1}\right)=n^{+}\left(V_{2}\right)$.

Proof. Let $k \in \operatorname{Adm}_{V_{1}} \cap \operatorname{Adm}_{V_{2}} \cap \widetilde{\operatorname{Adm}}$. For $x \in[a, b)$ we define

$$
\begin{aligned}
\mathcal{K}(x) & :=\left\{U \in \mathbb{S}^{n \times}:-\boldsymbol{\operatorname { R e }}(U J(k A(x)+B(x)))>0\right\}, \\
\mathcal{K}_{0}(x) & :=\left\{U \in \mathbb{S}^{n}:-\boldsymbol{\operatorname { R e }}(U J(k A(x)+B(x))) \geq 0\right\} .
\end{aligned}
$$

For a.e. $x \in[a, b)$ we have $V_{1}, V_{2} \in \mathcal{K}_{0}(x)$. Furthermore $\mathcal{K} \neq \emptyset$ on more than a set of measure zero. Hence there exists some $c \in[a, b)$, such that $\mathcal{K}(c) \neq \emptyset$ and $V_{1}, V_{2} \in \mathcal{K}_{0}(c)$. The statement follows by Lemma 6.1.5, using the regularity of $V_{1}$ and $V_{2}$.

If $A$ and $B$ have no common nulls-pace and $\operatorname{rank} A$ is large on a set of positive measure, the set $\widetilde{A d m}$ may be large. So this statement is a weaker version of Lemma 6.1.4. 
The previous statements in this section provide criteria according to which the set of admissible matrices is restricted. On the other hand, though, in the case that $B$ is selfadjoint on $[a, b)$, there is always the canonical choice of an admissible matrix:

Lemma 6.1.7. If $B$ is self-adjoint on $[a, b)$, then $V:= \pm i J$ is admissible with $0 \in A d m_{V}$ and $\mp i \in \Lambda_{V}$.

Proof. The formula $J J=-I_{n}$ and straightforward calculation gives

$$
C_{0, \pm i J}= \pm \mathbf{R e}(i B)=0
$$

and

$$
-\boldsymbol{R e}(\mp i( \pm i J J A))=A
$$

A little weaker premise is required in

Lemma 6.1.8. If $A \succcurlyeq-\operatorname{Im}(B)$ or $A \succcurlyeq \operatorname{Im}(B)$ then $V=-i J$, respectively $V=i J$ is admissible.

Proof. We only show the case $A \succcurlyeq-\mathbf{I m}(B)$. For $k>0$ such that $k A+\operatorname{Im}(B) \succcurlyeq 0$ we have

$$
C_{i k,-i J}=-\boldsymbol{\operatorname { R e }}(-i J J(i k A+B))=\boldsymbol{\operatorname { R e }}(k A-i B)=k A+\mathbf{I m}(B) \succcurlyeq 0
$$

and

$$
-\operatorname{Re}(i(-i J) J A)=A .
$$

Thus $i k \in \operatorname{Adm}_{V}$ and $i \in \Lambda_{V}$, whence $V=-i J$ is admissible.

We like to consider the scalar examples with regard to Lemma 6.1.8. We choose the even case where $A_{1}$ and $B_{1}$ are specified in (2.35) and (2.36).

To achieve $A_{1} \succcurlyeq \pm \mathbf{I m}\left(B_{1}\right)$, we can tighten the condition that $B$ is self-adjoint to

(6.3) $w \succcurlyeq \pm \operatorname{Im} p_{0}$ and for all $j \in\{1, \ldots, m\}$ holds $\bar{r}_{j}=-q_{j}, \pm \operatorname{Im} p_{j} \leq 0$ a.e. on $[a, b)$.

\subsection{On the wedges $\Lambda_{V}$ and the convex sets $W_{V}$}

In this section we examine the set $\Lambda_{V}$. It will turn out that two different circumstances may cause $\Lambda_{V}$ to remain within the upper or the lower half-plane.

The proof of the following linear algebraic Lemma is partially due to Ludwig Elsner. 
Lemma 6.2.1. For $K, P, N \in \mathbb{S}^{n}$ with $N>0$, the matrix $\operatorname{Im}(K P N)$ is either zero or indefinite, in particular it is not definite.

Proof. We can assume w.l.o.g. that $K$ is diagonal. Since $K$ and $P$ are self-adjoint, the diagonal entries are real and therewith so are the diagonal entries of $(K P)$, whence those entries of $\operatorname{Im}(K P)$ are zero. The trace of $\operatorname{Im}(K P)$, which is consequently zero, is the sum of all eigenvalues. Hence the statement holds for $N=I_{n}$.

For arbitrary positive definite $N \in \mathbb{S}^{n}$ let $\tilde{N}:=N^{1 / 2}$. We can calculate

$$
\operatorname{Im}(K P N)=\tilde{N} \operatorname{Im}\left(\left(\widetilde{N}^{-1} K \widetilde{N}^{-1}\right)(\tilde{N} P \widetilde{N})\right) \tilde{N}
$$

The righthand side is congruent to $\operatorname{Im}\left(\left(\widetilde{N}^{-1} K \widetilde{N}^{-1}\right)(\widetilde{N} P \widetilde{N})\right)$, which is is zero or indefinite, as already shown. Thus so is $\operatorname{Im}(K P N)$.

This simple Lemma leads to

Theorem 6.2.2. If $A$ is positive definite on a set of positive measure, then for arbitrary admissible $V$ holds

$$
\Lambda_{V} \cap \mathbb{R}=\emptyset
$$

Proof. Let $\lambda \in \mathbb{R}$ and let $\mathcal{J} \subset[a, b)$ the set, where $A$ is definite. We recall that $i J$ is self-adjoint. Using Lemma 6.2.1, we obtain for $x \in \mathcal{J}$ that

$$
-\boldsymbol{R e} \lambda V J A(x)=-\operatorname{Im}(i \lambda V J A(x))=-\mathbf{I m}(\lambda V)(i J) A(x)
$$

is not definite. (2.6) implies $\lambda \notin \Lambda_{V}$.

So if $A$ is definite, any of these open wedges $\Lambda_{V}$ is only a subset of the upper or the lower half-plane. On the other hand, in the case that $B$ is self-adjoint on $[a, b)$, we can achieve $\Lambda_{V}$ and $W_{V}$ to be any of both half-planes for the admissible choices of $V$ in Lemma 6.1.7:

Lemma 6.2.3. If $B$ is self-adjoint, then for $V:= \pm i J$ holds

$$
\Lambda_{V}=W_{V}=\{\lambda \in \mathbb{C}: \operatorname{Im} \lambda \lessgtr 0\} .
$$

Proof. We only show the case $V=-i J$. So let $\lambda \in \mathbb{C}$ with $y:=\operatorname{Im} \lambda>0$ and $x:=\operatorname{Re} \lambda$. The estimation

$$
-\boldsymbol{R e}(\lambda V J A)=\boldsymbol{R e}(i y i J J A)+\boldsymbol{R e}(x i J J A)=y \mathbf{R e} A-x \operatorname{Re}(i A)=y A \asymp A
$$

yields $\lambda \in \Lambda_{V}$ by (2.6). 
No $\lambda \in \mathbb{R}$ is in $\Lambda_{V}$, because

$$
-\boldsymbol{R e}(\lambda V J A)=\lambda \mathbf{R e}(i J J A)=\lambda \mathbf{R e}(-i A)=\lambda \mathbf{I m}(A)=0 \nsucc A .
$$

Since $\Lambda_{V}$ is connected, we obtain $\Lambda_{V}=\{\lambda \in \mathbb{C}: \operatorname{Im} \lambda>0\}$.

$0 \in \operatorname{Adm}_{V}$ by Lemma 6.1.7, thus $\operatorname{Adm}_{V} \supseteq \Lambda_{V}$. It suffices to show that any $k$ in the lower half-plane is not in $\mathrm{Adm}_{V}$. For such a $k$ holds:

$$
C_{k, V}=-\boldsymbol{\operatorname { R e }}((-i J) J(k A+B))=\operatorname{Re}(-i(k A+B))=\operatorname{Im}(k A)+\operatorname{Im} B=\operatorname{Im}(k) A \nsucceq 0 .
$$

Thus $k \notin \operatorname{Adm}_{V}$.

We have just discussed the shape of $\Lambda_{V}$. If $A>0$ on a set of positive measure, $\Lambda_{V}$ is contained in one of the halfplanes. Yet $W_{V}$, of which we know that it is a subset of $\rho(L)$, may be larger. Nevertheless we have to following

Lemma 6.2.4. If $A>0$ on a set of positive measure, then $W_{V}$ is contained in the (possibly shifted) upper or lower halfplane.

Proof. We already know that $\Lambda_{V}$ is contained in one of the halfplanes. We assume w.l.o.g. it is a subset of the upper halfplane. We show that $W_{V}$ is a subset of the upper halfplane plus some constant, which means $\operatorname{Im}\left(W_{V}\right)$ is bounded from below. It suffices to show that $\operatorname{Im}\left(\operatorname{Adm}_{V}\right)$ is bounded from below.

By assumption on the open set $\Lambda_{V}$, we can fix some $\lambda_{1}, \lambda_{2} \in \Lambda_{V}$ with $\operatorname{Im}\left(\lambda_{1}\right)=\operatorname{Im}\left(\lambda_{2}\right)=1$ and $k_{1}:=\operatorname{Re}\left(\lambda_{1}\right)<k_{2}:=\operatorname{Re}\left(\lambda_{2}\right)$.

We have $-\boldsymbol{R e}\left(\lambda_{l} V J A\right) \succcurlyeq A$ for $l \in\{1,2\}$, whence for a.e. $x \in\{\widetilde{x} \in[a, b): A(\widetilde{x})>0\}$ holds $-\boldsymbol{R e}\left(\lambda_{l} V J A(x)\right)>0$ for $l \in\{1,2\}$.

Let $\mathcal{J}$ the subset of $[a, b)$, where $A,-\mathbf{R e}\left(\lambda_{1} V J A\right)$, and $-\mathbf{R e}\left(\lambda_{2} V J A\right)$ are positive definite. $\mathcal{J}$ has positive measure.

We first show that, for $x \in \mathcal{J}$, the imaginary part of

$$
\mathcal{N}(x):=\left\{\lambda \in \mathbb{C}, C_{\lambda}(x) \geq 0\right\}
$$

is bounded from below. So let $x \in \mathcal{J}$.

For this proof, we use the abbreviation $\Xi:=\mathbf{R e}(V J B(x))$.

Any $\lambda_{0}=s i+t$ with $s, t \in \mathbb{R}$ lies in $\mathcal{N}(x)$ if and only if $C_{\lambda_{0}}(x) \geq 0$. This is equivalent to

$$
\Xi=\mathbf{R e}(V J B(x)) \leq-\boldsymbol{R e}\left(\lambda_{0} V J A(x)\right)=-s \mathbf{R e}(V(i J) A(x))-t \mathbf{I m}(V(i J) A(x)) .
$$

By Lemma 6.2.1, $\operatorname{Im}(V(i J) A(x))$ is either zero or indefinite. We distinguish these cases. 
First case: $\operatorname{Im}(V(i J) A(x))=0$.

We know $-\operatorname{Re}\left(\lambda_{1} V J A(x)\right)$ is positive definite, so there exists some $s_{0}<0$ such that $-s_{0} \operatorname{Re}\left(\lambda_{1} V J A(x)\right)<\Xi$. Hence for all $\lambda=i s+t$ with $s \leq s_{0}$

$$
\begin{aligned}
-s \boldsymbol{\operatorname { R e }}(V(i J) A(x))-t \operatorname{Im}(V(i J) A(x)) & =-s \mathbf{R e}(V(i J) A(x))-s k_{1} \mathbf{I m}(V(i J) A(x)) \\
& =-s \operatorname{Re}\left(\lambda_{1} V J A(x)\right) \\
& \leq-s_{0} \operatorname{Re}\left(\lambda_{1} V J A(x)\right)<\Xi,
\end{aligned}
$$

thus $\lambda_{0} \notin \mathcal{N}(x)$. So $\lambda \in \mathcal{N}(x)$ implies $\operatorname{Im}(\lambda)>s_{0}$.

Second case: $\operatorname{Im}(V(i J) A(x))$ is indefinite.

Then there exist $j_{1}<0<j_{2}$, such that for all $j \in \mathbb{R} \backslash\left[j_{1}, j_{2}\right]$ the matrix $j \operatorname{Im}(V(i J) A(x))-\Xi$ is not positive semi definite.

For $\lambda_{0}=s i+t$ with $s<-\frac{j_{2}-j_{1}}{k_{2}-k_{1}}$ we have $t-s k_{1}<j_{1}$ or $t-s k_{2}>j_{2}$.

For $l \in\{1,2\}$, using $\lambda_{l}=k_{l}+i$, we can estimate

$$
\begin{aligned}
C_{\lambda_{0}}(x) & =-\operatorname{Re}(V J B(x))-\operatorname{Re}\left(V J \lambda_{0} A(x)\right)=-\Xi-\operatorname{Re}\left(V J \lambda_{0} A(x)\right) \\
& <-\Xi-\operatorname{Re}\left(V J \lambda_{0} A(x)\right)-(-s) \operatorname{Re}\left(V J \lambda_{l} A(x)\right) \\
& =-\Xi-\operatorname{Re}\left(V J\left(t-s k_{l}\right) A(x)\right) \\
& =-\Xi+\left(t-s k_{l}\right) \operatorname{Im}(V(i J) A(x)) .
\end{aligned}
$$

At least once $\left(t-s k_{l}\right) \notin\left[j_{1}, j_{2}\right]$, whence the righthand sides is not positive semi definite. Thus $\lambda_{0} \notin \mathcal{N}(x)$. So $\lambda \in \mathcal{N}(x)$ implies $\operatorname{Im}(\lambda)>-\frac{j_{2}-j_{1}}{k_{2}-k_{1}}$.

Since the imaginary part of $\mathcal{N}(x)$ is bounded from below for every $x \in \mathcal{J}$, there is a function $f: \mathcal{J} \rightarrow \mathbb{R}$, such that for every $x \in \mathcal{J}$ and every $\lambda$ with $C_{\lambda}(x) \geq 0$ holds $\operatorname{Im}(\lambda) \geq f(x)$. Since $\mathcal{J}$ has positive measure, there exists some $f_{0} \in \mathbb{R}$, such that $f^{-1}\left(\left[f_{0}, \infty\right]\right)$ is not a set of measure zero. (Otherwise $\mathcal{J}=\cup\left\{f^{-1}([-j, \infty]): j \in \mathbb{N}\right\}$ would be of measure zero.)

For any $\lambda \in \operatorname{Adm}_{V}$ holds $C_{\lambda}(x) \geq 0$ for almost every $x \in[a, b)$, in particular for a.e. $x \in \mathcal{J}$. This finally implies $\operatorname{Im}(\lambda) \geq f_{0}$. Thus the Theorem follows.

\subsection{An augmentation method}

In this chapter we introduce a method to augment the problem to one of higher order which remains equivalent to the original problem. Though this seems to be of no real importance, it provides an insight into some questions of possibility. 
Let $m^{+}, m^{-} \in \mathbb{N} \cup\{0\}$. These are the dimensions by which we will augment the problem.

We assume we have chosen some suitable $(V, E)$ with corresponding $n^{+}=n^{+}(V), n^{-}=$ $n^{-}(V)$.

First we declare some abbreviations:

We set

$$
\widetilde{n}:=n+m^{-}+m^{+}, \quad \widetilde{n}^{-}:=n^{-}+m^{-}, \quad \tilde{n}^{+}:=n^{+}+m^{+} .
$$

For matrices $N \in \mathbb{C}^{n, n}, P^{-} \in \mathbb{C}^{m^{-}, m^{-}}, P^{+} \in \mathbb{C}^{m^{+}, m^{+}}, Q^{-} \in \mathbb{C}^{n^{-}, n^{-}}, Q^{+} \in \mathbb{C}^{n^{+}, n^{+}}$,

$\Theta \in \mathbb{C}^{n, n^{-}}, \Phi \in \mathbb{C}^{n, n^{+}}$and $T \in \mathbb{C}^{n^{-}, n^{+}}$we use the notations

$$
\begin{array}{rlrl}
{\left[N, P^{-}, P^{+}\right]} & :=\operatorname{diag}\left(P^{-}, N, P^{+}\right) \in \mathbb{C}^{\widetilde{n}, \widetilde{n}}, \\
\underline{\overline{|N|}} & :=\left[N, 0_{m^{-}}, 0_{m^{+}}\right], \\
\left(Q^{-}, P^{-}\right)^{-} & :=\left(\begin{array}{cc}
P^{-} & 0 \\
0 & Q^{-}
\end{array}\right) \in \mathbb{C}^{\widetilde{n}^{-}, \widetilde{n}^{-}}, \quad\left[\Theta, P^{-}\right]^{-}:=\left(\begin{array}{cc}
P^{-} & 0 \\
0 & \Theta \\
0 & 0
\end{array}\right) \in \mathbb{C}^{\widetilde{n}, \widetilde{n}^{-}}, \\
\left(Q^{+}, P^{+}\right)^{+} & :=\left(\begin{array}{cc}
Q^{+} & 0 \\
0 & P^{+}
\end{array}\right) \in \mathbb{C}^{\widetilde{n}^{+}, \widetilde{n}^{+},} \quad\left[\Phi, P^{+}\right]^{+}:=\left(\begin{array}{cc}
0 & 0 \\
\Phi & 0 \\
0 & P^{+}
\end{array}\right) \in \mathbb{C}^{\widetilde{n}, \widetilde{n}^{+},} \\
\overline{T \mid} & :=\left(\begin{array}{cc}
0 & 0 \\
T & 0
\end{array}\right) \in \mathbb{C}^{\widetilde{n}^{-}, \widetilde{n}^{+},} & \underline{\mid T^{*}}: & :=\left(\begin{array}{cc}
0 & T^{*} \\
0 & 0
\end{array}\right) \in \mathbb{C}^{\widetilde{n}^{+}, \widetilde{n}^{-}} .
\end{array}
$$

Note that in each of these abbreviative functions each first argument on the left side will be a function (or constant) of the original problem.

For matrices with adequate dimensions, the following holds:

$$
\begin{gathered}
{\left[\left(\begin{array}{cc}
Q^{-} & T \\
U & Q^{+}
\end{array}\right), P^{-}, P^{+}\right]=\left(\begin{array}{cc}
\left(Q^{-}, P^{-}\right)^{-} & \overline{T \mid} \\
\underline{\mid U} & \left(Q^{+}, P^{+}\right)^{+}
\end{array}\right),} \\
{\left[N, P^{-}, P^{+}\right]^{*}=\left[N^{*}, P^{-*}, P^{+*}\right],} \\
{\left[N, P^{-}, P^{+}\right]\left[\widetilde{N}, \widetilde{P^{-}}, \widetilde{P^{+}}\right]=\left[N \widetilde{N}, P^{-} \widetilde{P^{-}}, P^{+} \widetilde{P^{+}}\right],} \\
{\left[N, P^{-}, P^{+}\right]\left[\Phi, \widetilde{P^{+}}\right]^{+}=\left[N \Phi, P^{+} \widetilde{P^{+}}\right]^{+}, \quad \underline{|N|}\left[\Phi, P^{+}\right]^{+}=[N \Phi, 0]^{+},} \\
\left(Q^{+}, P^{+}\right)^{+} \underline{\mid U}=\underline{\mid Q^{+} U}, \quad \overline{T \mid}\left(Q^{-}, P^{-}\right)^{-}=\overline{T Q^{-} \mid},
\end{gathered}
$$




$$
\overline{T \mid} \underline{\mid U}=(T U, 0)^{-}, \quad \underline{\mid U} \overline{T \mid}=(U T, 0)^{+},
$$

$$
\left[\Phi, P^{+}\right]^{+} \underline{\mid U}=[\Phi U, 0]^{-} .
$$

We want to pose the augmented problem. Therefor let $J^{-} \in \mathbb{C}^{m^{-}, m^{-}}, J^{+} \in \mathbb{C}^{m^{+}, m^{+}}$such that $i J^{-}$and $i J^{+}$are unitary and self-adjoint. We mark the corresponding matrices for the augmented problem in this section with a tilde.

Let $\widetilde{A}:=\overline{|A|}, \widetilde{B}:=\overline{|B|}, \widetilde{J}:=\left[J, J^{-}, J^{+}\right]$. The formal augmented problem reads

$$
\widetilde{J} \widetilde{y}_{\lambda}^{\prime}(x)=(\lambda \widetilde{A}(x)+\widetilde{B}(x)) \widetilde{y}_{\lambda}(x)
$$

For $\widetilde{E}:=\left[E, I_{m^{-}}, I_{m^{+}}\right]$and $\widetilde{V}:=\left[V,-I_{m^{-}}, I_{m^{+}}\right]$, we obtain

Lemma 6.3.1. The pair $(\widetilde{V}, \widetilde{E})$ is suitable for the augmented problem with

$$
\begin{gathered}
n^{-}(\widetilde{V})=\widetilde{n}^{-}, \quad n^{+}(\widetilde{V})=\widetilde{n}^{+}, \\
\widetilde{A d m_{V}}=A d m_{V}, \quad \widetilde{\Lambda_{V}}=\Lambda_{V}, \quad \widetilde{C_{\lambda}}=\underline{\mid \overline{C_{\lambda} \mid}}, \\
\widetilde{Y_{\lambda}}=\left[Y_{\lambda}, I_{m^{-}}, I_{m^{+}}\right], \quad \widetilde{\Theta_{\lambda}}=\left[\Theta_{\lambda}, I_{m^{-}}\right]^{-}, \quad \widetilde{\Phi_{\lambda}}=\left[\Phi_{\lambda}, I_{m^{+}}\right]^{+}, \\
\widetilde{S_{\lambda}}=\left(S_{\lambda},-\frac{1}{2} I_{m^{-}}\right)^{-}, \quad \widetilde{P_{\lambda}}=\left(P_{\lambda}, \frac{1}{2} I_{m^{+}}\right)^{+}, \widetilde{T_{\lambda}}=\overline{T_{\lambda} \mid}, \quad{\widetilde{T_{\lambda}}}^{*}=\underline{T_{\lambda}^{*}}, \\
\widetilde{\mathcal{C}_{\lambda}}=\underline{\mid \mathcal{C}_{\lambda}}, \quad \widetilde{\mathcal{R}_{\lambda}}=\left(\mathcal{R}_{\lambda}, \frac{1}{2} I_{m^{-}}\right)^{-} .
\end{gathered}
$$

Corresponding statements hold for the respective functions of the adjoint problem. It should be mentioned that the shape of $\widetilde{Z_{\lambda}}, \widetilde{\eta_{\lambda}}$ and $\widetilde{\chi_{\lambda}}$ is as follows:

$$
\widetilde{Z_{\lambda}}=\left(\widetilde{\eta_{\lambda}} \mid \widetilde{\chi_{\lambda}}\right) \text { with } \widetilde{\eta_{\lambda}}=\left(\begin{array}{cc}
0 & 0 \\
\eta_{\lambda} & 0 \\
0 & -J^{+}
\end{array}\right) \text {and } \widetilde{\chi_{\lambda}}=\left(\begin{array}{cc}
J^{-} & 0 \\
0 & \chi_{\lambda} \\
0 & 0
\end{array}\right) \text {. }
$$


Proof. The calculations for $\widetilde{n}^{-}, \widetilde{n}^{+}, \widetilde{C_{\lambda}}, \widetilde{Y_{\lambda}}, \widetilde{\Theta_{\lambda}}$ and $\widetilde{\Phi_{\lambda}}$ are straightforward.

Therewith obviously $\widetilde{C_{\lambda, V}} \succcurlyeq 0$ if and only if $C_{\lambda, V} \succcurlyeq 0$ and thus holds $\widetilde{\operatorname{Adm}_{V}}=\operatorname{Adm}_{V}$.

Furthermore $\widetilde{V} \widetilde{J} \widetilde{A}=\underline{|V J A|}$ by (6.8), and so (2.6) yields $\widetilde{\Lambda_{V}}=\Lambda_{V}$. Since admissibility of $V$ is equivalent to these sets not being empty we conclude that $\widetilde{V}$ is admissible.

(2.18), (6.7), and (6.8) yield

$$
2\left(\begin{array}{cc}
\widetilde{S} & \widetilde{T} \\
\widetilde{T}^{*} & \widetilde{P}
\end{array}\right)=\widetilde{Y}^{*} \widetilde{V} \widetilde{Y}=\left[Y^{*} V Y,-I, I\right]=\left[2\left(\begin{array}{cc}
S & T \\
T^{*} & P
\end{array}\right),-I, I\right] .
$$

Hence (6.6) implies the statements for $\widetilde{S}, \widetilde{P}$ and $\widetilde{T}$.

Since $P(a) \geq 0$, we know $\widetilde{P}(a) \geq 0$. For any $\xi=\left(\begin{array}{l}\xi_{1} \\ \xi_{2}\end{array}\right) \in \mathbb{C}^{\widetilde{n}^{+}}$with $\xi_{1} \in \mathbb{C}^{n^{+}}, \xi_{2} \in \mathbb{C}^{m^{+}}$, which fulfills the premise for the definiteness assumption (2.10) for the augmented problem, (6.17) yields $\xi_{2}=0$ and $\xi_{1}$ fulfills the same premise for the original problem and therefore is zero. It follows that $(\widetilde{V}, \widetilde{E})$ is suitable.

The statements for $\widetilde{\mathcal{C}_{\lambda}}$ and $\widetilde{\mathcal{R}_{\lambda}}$ hold by $(2.24),(2.25),(6.10)$, and $(6.11)$.

We fix the suitable pair $(\widetilde{V}, \widetilde{E})$. With equations $(2.26),(6.10),(6.11),(6.17)$, and $(6.18)$ we obtain

$$
\widetilde{D_{\lambda}}(x) \supseteq\left\{\underline{\mid l}: l \in D_{\lambda}(x)\right\} .
$$

The choice $\widetilde{\mu}=\mu$ and $\widetilde{M}_{0}:=\mid M_{0} \in \widetilde{D_{\lambda}}(b)$ yields $\widetilde{M}=\mid M$ by straightforward (yet technical) calculations (the crucial equations in the construction of the $M$-function are (4.14) and (4.25)). This leads to

$$
\widetilde{\Psi_{\lambda}}=\left[\Psi_{\lambda}, I_{m^{-}}\right]^{-}=\left(\begin{array}{cc}
I_{m^{-}} & 0 \\
0 & \Psi_{\lambda} \\
0 & 0
\end{array}\right) \text { and } \widetilde{\zeta_{\lambda}}=\left(\begin{array}{cc}
0 & 0 \\
\zeta_{\lambda} & 0 \\
0 & -J^{+}
\end{array}\right)
$$

For $\tilde{f} \in L_{\widetilde{A}}^{2}[a, b)$ let $f:=\left(f_{m^{-}+1}, \ldots, f_{m^{-}+n}\right)^{T}$. If $\tilde{f}$ was not zero in $L_{\widetilde{A}}^{2}[a, b)$, then $f$ is not zero in $L_{A}^{2}$ by construction of $\widetilde{A}$. Again by straightforward calculation, using (4.34) and (4.35), we obtain

$$
\widetilde{R_{\lambda}} \widetilde{f}=(\underbrace{0, \ldots, 0}_{m^{-}}, R_{\lambda} f, \underbrace{0, \ldots, 0}_{m^{+}})^{T} \text { and } \widetilde{\widehat{R}_{\lambda}} \widetilde{f}=(\underbrace{0, \ldots, 0}_{m^{-}}, \widehat{R}_{\lambda} f, \underbrace{0, \ldots, 0}_{m^{+}})^{T},
$$

which shows that Assumption 2 of the augmented problem is fulfilled, as long as it is fulfilled in the original problem.

The theory finally yields the operator $\widetilde{L}$. With

$$
\begin{array}{r}
D(\widetilde{L})=\left\{\widetilde{y} \in L_{\widetilde{A}}^{2} \cap A C_{\mathrm{loc}}[a, b):{\widetilde{\chi_{\mu}}}^{*}(a) \widetilde{J} \widetilde{y}(a)=0=\lim _{x \rightarrow b} \widetilde{\zeta}_{\mu}^{*}(x) \widetilde{J} \widetilde{y}(x)\right. \\
\text { and } \left.\widetilde{J} \widetilde{y}^{\prime}-\widetilde{B} \widetilde{y}=\widetilde{A} \widetilde{f} \text {, for some } \widetilde{f}=: \widetilde{L} \widetilde{y} \in L_{\widetilde{A}}^{2}\right\} .
\end{array}
$$


Let $\mathcal{I}$ the projection

$$
\left([a, b) \rightarrow \mathbb{C}^{\widetilde{n}}\right) \rightarrow\left([a, b) \rightarrow \mathbb{C}^{n}\right), \quad \mathcal{I}\left(\left(f_{1}, \ldots, f_{\widetilde{n}}\right)^{T}\right):=\left(f_{m^{-}+1}, \ldots, f_{m^{-}+n}\right)^{T}
$$

The construction of $\widetilde{A}$ yields that $\mathcal{I}$ maps $L_{\widetilde{A}}^{2}$ onto $L_{A}^{2}$, bijectively, isometrically, and isomorphically.

Straight calculations yields that for any $\widetilde{y} \in D(\widetilde{L})$ and any $y \in D(L)$ holds

$\mathcal{I}(\widetilde{y}) \in D(L)$ with $L \mathcal{I}(\widetilde{y})=\mathcal{I}(\widetilde{L} \widetilde{y})$ and $\mathcal{I}^{-1} y \in D(\widetilde{L})$ with $\widetilde{L} \mathcal{I}^{-1}(y)=\mathcal{I}^{-1}(L y)$. (Recall that the assumptions on $\widetilde{y}$ to be in $D(\widetilde{L})$ is posed for one representative of this element $\widetilde{y}$, which is a class of functions.)

Thus we obtain

\section{Corollary 6.3.2.}

$$
\widetilde{L}=\mathcal{I}^{-1} L \mathcal{I} \quad \text { and } \quad L=\mathcal{I} \widetilde{L} \mathcal{I}^{-1}
$$

as operators in $L_{\widetilde{A}}^{2}$, respectively in $L_{A}^{2}$.

By (6.15) we do not have any better approximation of the spectrum of $L$. Yet the augmentation method shows some things:

With an appropriate choice of $m^{-}, m^{+}, J^{-}$and $J^{+}$, such that $n^{-}(i \widetilde{J})=n^{+}(i \widetilde{J})$ and $\widetilde{n}^{-}=\widetilde{n}^{+}$, and with an appropriate basis transform (such that $\widetilde{J}=\operatorname{codiag}\left(I_{m^{-}},-I_{m^{-}}\right)$), the augmented problem fulfills the assumptions in [5], furthermore $\mathcal{U}_{2 n}:=-\widetilde{V} \widetilde{J}$ is a suitable matrix for the theory therein fulfilling all necessary assumptions. Thus the augmentation method may transform the original problem into an even order problem (where even further assumptions are fulfilled).

Since $\widetilde{P}=\left(P, \frac{1}{2} I_{m^{+}}\right)^{+}$, we know that in the augmented problem, additionally $m^{+}$eigenvalues of $\widetilde{P}(x)$ remain bounded as $x$ tends to $b$. Thus Lemma 5.1.2 states that in the augmented problem, $m^{+}$more linearly independent solutions of (6.13) exist that are in $L_{\widetilde{C_{\lambda}}}$.

Yet $\widetilde{C_{\lambda}}=\overline{\left|C_{\lambda}\right|}$ and thus $\mathcal{I}$ maps $L_{\widetilde{C_{\lambda}}}^{2}$ onto $L_{C_{\lambda}}^{2}$ isometrically and isomorphically. For any solution $\widetilde{y} \in L_{\widetilde{C_{\lambda}}}^{2}$ of the augmented version of (2.1) we know $\mathcal{I} \widetilde{y}$ is a solution of (2.1). So those $m^{+}$further linearly independent solutions are not linearly independent within $L_{\widetilde{C_{\lambda}}}^{2}$. This shows that we really have to distinguish between linearly independence as functions $[a, b) \rightarrow \mathbb{C}^{n}$ and linearly independence in $L_{C_{\lambda}}^{2}$.

Another conclusion is the following: If both $m^{-}$and $m^{+}$are not zero, (6.17) and a corresponding statement for $\widetilde{P}$ shows that of both, $\widetilde{P}(x)$ and $\widetilde{P}(x)$, at least one eigenvalue remains bounded (as $x$ tends to $b$ ). Using Lemma 5.1.2 and an analogous statement for the adjoint 
problem, Theorem 5.1.14 yields that (2.1) is in the non-limit-point case. Thus we can change from limit-point case to non-limit-point case without changing the problem essentially (in view of Corollary 6.3.2).

Since in the non-limit-point case we have better approximations of the number of linearly independent solutions of $(2.1)$ that are in $L_{C_{\lambda}}^{2}$, we can use the augmentation method to obtain

Lemma 6.3.3. Let $(V, E)$ suitable for (2.1). We augment the problem by one dimension, choosing $m^{+}:=0, m^{-}:=1, J^{-}:=i$.

For $\tilde{\mathbf{r}}$, the value that corresponds to $\mathbf{r}$ (see (5.3)) for the augmented problem, holds: There are exactly $n^{-}+\tilde{\mathbf{r}}$ independent solutions of (2.1) that are in $L_{C_{\lambda}}^{2}$.

Proof. Using $\widetilde{\mathcal{R}_{\lambda}}=\left(\mathcal{R}_{\lambda}, \frac{1}{2}\right)^{-}$, equation (3.47) yields that at least one eigenvalue of $\widetilde{P}(x)$ remains bounded as $x$ tends to $b$. Thus there are more than $\widetilde{n}^{+}$independent solutions of the augmented version of (3.15) that are in $L_{\widetilde{C_{\lambda}}}^{2}$ by the dual version of Lemma 5.1.2 for the augmented problem.

Hence, if the augmented problem is in the limit-point case, Theorem 5.1.14 implies that there are exactly $\widetilde{n}^{-}=1+n^{-}$solutions of the augmented version of $(2.1)$ that are in $L_{\widetilde{C_{\lambda}}}^{2}$. Furthermore holds $\tilde{\mathbf{r}}=0$ in the limit-point case by definition.

If the augmented problem is in the in the non-limit-point case, Theorem 5.1.10 yields that there are exactly $\widetilde{n}^{-}+\tilde{\mathbf{r}}=n^{-}+1+\tilde{\mathbf{r}}$ solutions of the augmented version of (2.1) that are in $L_{\widetilde{C_{\lambda}}}^{2}$.

In both cases there are exactly $n^{-}+1+\tilde{\mathbf{r}}$ solutions of the augmented version of (2.1) that are in $L_{\widetilde{C_{\lambda}}}^{2}$.

The number of linearly independent solutions of (2.1) that are in $L_{C_{\lambda}}^{2}$ is exactly one less, than the corresponding number in the augmented problem (with the further solution $y \equiv(1,0, \ldots, 0)^{T}$, which is even zero in $\left.L_{\widetilde{C_{\lambda}}}^{2}\right)$. Hence the statement follows.

If the original problem is in the limit-point case, then the geometrical quantity $\mathbf{r}$ does not yield the amount of linearly independent solutions of (2.1) (in view of Theorem 5.1.10). After appropriate augmentation, the resulting quantity $\tilde{\mathbf{r}}$ does by Lemma 6.3.3. 


\subsection{The case $n=1$}

We finally consider the case that $n$ is one.

The one-dimensional problem has the advantage that we can calculate the solutions of (2.1) explicitely. Even this rather simple case offers an insight into some questions of possibility.

With $a:=0, b:=\infty, J:=-i, A \in L_{\text {loc }}^{1}\left([0, \infty), \mathbb{R}_{0}^{+}\right), B \in L_{\text {loc }}^{1}([0, \infty), \mathbb{C})$, problem $(2.1)$ reads

$$
-i y^{\prime}=(\lambda A+B) y
$$

The alternative choice $J=i$ would create a similar problem.

The only possible choices for $V$ (up to an irrelevant positive constant) is \pm 1 so $\left(n^{+}, n^{-}\right) \in$ $\{(1,0),(0,1)\}$.

Therewith

$$
\Lambda_{V}=\{\lambda \in \mathbb{C}: \operatorname{Re}( \pm i \lambda A) \succcurlyeq A\}=\{\lambda \in \mathbb{C}: \mp \operatorname{Im}(\lambda A) \succcurlyeq A\}=\{\lambda \in \mathbb{C}: \operatorname{Im}(\lambda) \lessgtr 0\},
$$

the lower, respectively the upper half-plane. Since

$$
C_{k, V}=\operatorname{Re}( \pm i(k A+B))=\mp \operatorname{Im}(k A+B),
$$

we have

$$
\operatorname{Adm}_{V}=\{k \in \mathbb{C}: \mp(\operatorname{Im}(k) A+\operatorname{Im}(B)) \succcurlyeq 0\} .
$$

Thus Assumption 1 reads

$$
A \succcurlyeq \operatorname{Im}(B) \quad \text { or } \quad A \succcurlyeq-\operatorname{Im}(B) \text {. }
$$

Assumption 2 holds always true by Remark 4.4.2.

In view of Theorem 5.4.1, we furthermore have $\widehat{b}=\infty$ by Corollary 5.4.3.

We distinguish three possible cases:

$$
\begin{array}{lll}
\text { case 1: } A \succcurlyeq-\operatorname{Im}(B) & \text { and } & A \succcurlyeq \mathbf{I m}(B), \\
\text { case 2: } A \succcurlyeq-\operatorname{Im}(B) & \text { and } & A \succcurlyeq \mathbf{I m}(B), \\
\text { case 3: } A \succcurlyeq-\operatorname{Im}(B) & \text { and } & A \succcurlyeq \mathbf{I m}(B) .
\end{array}
$$

By Remark 2.1.4, the choice $E=1$ does not restrict the generality of our examination; using additionally Lemma 2.1.3, we see that for an admissible $V$, the pair $(V, E)$ is suitable.

$Y_{\lambda}$ solves $Y_{\lambda}^{\prime}=i(\lambda A+B) Y_{\lambda}$ and $Y_{\lambda}(0)=1$. Thus we have for all $c \in[0, \infty)$ 


$$
\begin{aligned}
Y_{\lambda}(c) & =\exp \left(i \int_{0}^{c} \lambda A+B d x\right) \\
& =\exp \left(i \int_{0}^{c} \operatorname{Re}(\lambda) A+\boldsymbol{R e}(B) d x\right) \quad \exp \left(-\int_{0}^{c} \operatorname{Im}(\lambda) A+\mathbf{I m}(B) d x\right) .
\end{aligned}
$$

The formal adjoint problem (3.15) reads

$$
z_{\lambda}^{\prime}=i(\bar{\lambda} A+\bar{B}) z_{\lambda}
$$

Remark 6.4.1. Since $\operatorname{Im}(\bar{B})=-\operatorname{Im}(B)$, for the adjoint problem, the cases 1 and 2 interchange. Furthermore $\widehat{V}=-V$ by (3.16).

For $V \in\{ \pm 1\}$, by (3.18) and (3.17) we obtain $J_{0}=V$ and $\widehat{E}=i V$. Thus we have for all $c \in[0, \infty)$

$$
Z_{\lambda}(c)=i V \exp \left(i \int_{0}^{c} \operatorname{Re}(\lambda) A+\mathbf{R e}(B) d x\right) \quad \exp \left(\int_{0}^{c} \mathbf{I m}(\lambda) A+\mathbf{I m}(B) d x\right) .
$$

First we assume $A \succcurlyeq-\operatorname{Im}(B)$, so we are in case 1 or 3 .

Let $k_{-1}:=\inf \{k \in \mathbb{R}: k A+\operatorname{Im}(B) \succcurlyeq 0\}$.

The choice $V:=-1$ is admissible with $\left(n^{+}, n^{-}\right)=(0,1)$ and

$$
\operatorname{Adm}_{-1}=\left\{\lambda \in \mathbb{C}: \operatorname{Im} \lambda \geq k_{-1}\right\} .
$$

So we have

$$
W_{-1}=\left\{\lambda \in \mathbb{C}: \operatorname{Im} \lambda>k_{-1}\right\} .
$$

With $n^{+}=0$ and $n^{-}=1$ we obtain $\Theta_{\lambda}=Y_{\lambda}, \Phi_{\lambda}=0_{1,0}, \eta_{\lambda}=0_{1,0}$ and $\chi_{\lambda}=Z_{\lambda}$.

Because $P_{\lambda,-1}(c) \in \mathbb{C}^{n^{+}, n^{+}}=\mathbb{C}^{0,0}$ we have $P_{\lambda,-1}=0_{0,0}$ on $[0, \infty)$. This no eigenvalues.

Thus no eigenvalue of $P_{\lambda,-1}$ is bounded. By Lemma 5.1.2 there is a non-trivial solution of (2.1) that lies in $L_{C_{\lambda}}^{2}$. So $Y_{\lambda} \in L_{C_{\lambda}}^{2} \subseteq L_{A}^{2}$ for $\lambda \in W_{-1}$. We know the explicit shape of $Y_{\lambda}$, so this can already be checked in (6.24) with fundamental analysis means, using

$$
\operatorname{Im}(\lambda) A+\operatorname{Im}(B)=k_{-1} A+\operatorname{Im}(B)+\left(\operatorname{Im}(\lambda)-k_{-1}\right) A \succcurlyeq\left(\operatorname{Im}(\lambda)-k_{-1}\right) A \succcurlyeq A
$$


Since $\emptyset \neq D_{\lambda,-1}(\infty) \subseteq \mathbb{C}^{n^{+}, n^{-}}$we can conclude $D_{\lambda,-1}(\infty)=\mathbb{C}^{n^{+}, n^{-}}=\left\{0_{0,1}\right\}$, so only the limit-point case occurs with $M \equiv 0_{0,1}$, and hence

$$
\begin{aligned}
\Psi_{\lambda} & =\Theta_{\lambda}+\Phi_{\lambda} M=Y_{\lambda}+0=Y_{\lambda}, \\
\zeta_{\lambda} & =\eta_{\lambda}+\chi_{\lambda} M^{*}=\eta_{\lambda}=0_{1,0} .
\end{aligned}
$$

With (4.36) and (4.35), we obtain

$$
\begin{aligned}
& R_{\lambda} f(x)=Y_{\lambda}(x) \int_{0}^{x} \overline{Z_{\lambda}}(y) A(y) f(y) d y \\
& \widehat{R}_{\lambda} f(x)=Z_{\lambda}(x) \int_{x}^{\infty} \overline{Y_{\lambda}}(y) A(y) f(y) d y .
\end{aligned}
$$

The domain of definition of the operator $L_{-1}$ is given by

$$
D\left(L_{-1}\right)=\left\{y \in L_{A}^{2} \cap A C_{\mathrm{loc}}[0, \infty): \exists f \in L_{A}^{2}: A f=-i y^{\prime}-B y \text { a.e. and } y(0)=0\right\}
$$

Before considering further results, we examine the other possible choice of $V$.

We now assume $A \succcurlyeq \operatorname{Im}(B)$, so we are in case 2 or 3 .

Let $k_{1}:=\sup \{k \in \mathbb{R}:-k A-\operatorname{Im}(B) \succcurlyeq 0\}$.

The choice $V:=1$ is admissible with $\left(n^{+}, n^{-}\right)=(1,0)$ and

$$
\operatorname{Adm}_{1}=\left\{k \in \mathbb{C}: \operatorname{Im} \lambda \leq k_{1}\right\}
$$

So we obtain

$$
W_{1}=\left\{\lambda \in \mathbb{C}: \operatorname{Im} \lambda<k_{1}\right\}
$$

With these values of $n^{+}$and $n^{-}$we obtain $\Theta_{\lambda}=0_{1,0}, \Phi_{\lambda}=Y_{\lambda}, \eta_{\lambda}=Z_{\lambda}$ and $\chi_{\lambda}=0_{1,0}$.

Again (2.1) is in the limit-point case, now with $D_{\lambda, 1}(\infty)=\mathbb{C}^{n^{+}, n^{-}}=\left\{0_{1,0}\right\}$. Hence $M \equiv 0_{1,0}$ on $W_{1}$ and therewith

$$
\begin{aligned}
\Psi_{\lambda} & =\Theta_{\lambda}+\Phi_{\lambda} M=0_{1,0}, \\
\zeta_{\lambda} & =\eta_{\lambda}+\chi_{\lambda} M^{*}=\eta_{\lambda}=Z_{\lambda} .
\end{aligned}
$$


For $\lambda \in W_{1}$ holds

$$
\begin{aligned}
P_{\lambda, 1} & =Y_{\lambda}^{*} V Y_{\lambda}=\overline{Y_{\lambda}} Y_{\lambda}=\exp \left(-2 \int_{0}^{c} \operatorname{Im}(\lambda) A+\operatorname{Im}(B) d x\right) \\
& =\exp \left(\nu \int_{0}^{c} A d x\right) \quad \exp \left(2 \int_{0}^{c}-k_{1} A-\operatorname{Im}(B) d x\right),
\end{aligned}
$$

with $\nu:=-2\left(\operatorname{Im}(\lambda)-k_{1}\right)>0$.

Lemma 5.1.2 yields that $Y_{\lambda} \in L_{C_{\lambda}}^{2}$ if and only if $P_{\lambda, 1}(c)$ is bounded as $c$ tends to infinity. Both integrands (and therewith both brackets) on the right-hand side of (6.31) are nonnegative. Therewith we obtain

Remark 6.4.2. $Y_{\lambda} \in L_{C_{\lambda}}^{2}$ if and only if $A, \operatorname{Im}(B) \in L^{1}(0, \infty)$.

If this is not the case, we only know that $Y_{\lambda}$ is not in $L_{C_{\lambda}}^{2}$. The theory does not state that $Y_{\lambda}$ may not be in $L_{A}^{2}$. So we can distinguish three sub-cases:

$$
\begin{array}{ll}
\text { sub-case } \mathbf{a}: & Y_{\lambda} \in L_{C_{\lambda}}^{2}, \\
\text { sub-case b: } & Y_{\lambda} \in L_{A}^{2} \text { and } Y_{\lambda} \notin L_{C_{\lambda}}^{2}, \\
\text { sub-case c: } & Y_{\lambda} \notin L_{A}^{2},
\end{array}
$$

for some $\lambda \in W_{1}$. (By Theorem 5.1.6, the actual choice of this $\lambda \in W_{1}$ is irrelevant.)

In case 3, using $A \succcurlyeq-\operatorname{Im} B$ and (6.21), we obtain for $\lambda \in W_{1}$

$$
C_{\lambda, 1}=-\operatorname{Im}(\lambda) A-\operatorname{Im}(B) \preccurlyeq A,
$$

which shows $C_{\lambda, 1} \asymp A$. Hence only sub-cases $\mathbf{a}$ and $\mathbf{c}$ are possible in case $\mathbf{3}$.

The operator $L_{1}$ has the domain of definition

$D\left(L_{1}\right)=\left\{y \in L_{A}^{2} \cap A C_{\mathrm{loc}}[0, \infty): \exists f \in L_{A}^{2}: A f=-i y^{\prime}-B y\right.$ a.e. and $\left.\lim _{x \rightarrow \infty} Z_{\mu}(x) y(x)=0\right\}$.

Using (4.62), in sub-case $\mathbf{c}$ we obtain

$$
D\left(L_{1}\right)=\left\{y \in L_{A}^{2} \cap A C_{\mathrm{loc}}[0, \infty): \exists f \in L_{A}^{2}: A f=-i y^{\prime}-B y \text { a.e. }\right\} .
$$

In case 3.c (where also $L_{-1}$ exists), this is a proper superset of $D\left(L_{-1}\right)$.

On the intersection of their domains, both operators are equal. Thus we obtain 
Corollary 6.4.3. In case 3.c, the operator $L_{1}$ is a proper extension of $L_{-1}$ and hence their resolvent sets are disjoint.

We have not yet shown that all these cases are really possible. To fill this gap, we regard some simple concrete examples.

For case 1 we could choose $A(x):=1$ and $B(x):=i x$.

For case 2.a, the choices $A(x):=(x+1)^{-3}$ and $B(x):=-i(x+1)^{-2}$ will do by Remark 6.4.2.

The choice $A(x):=(x+1)^{-4}$ and $B(x):=-i(x+1)^{-1}$ is obviously in case 2.

Remark 6.4.2 yields $Y_{\lambda} \notin L_{C_{\lambda}}^{2}$, since $\operatorname{Im}(B) \notin L^{1}(0, \infty)$.

With this choice of $A$ and $B$, we obtain $k_{1}=1$. Thus we have $\lambda=0 \in W_{1}$. For this $\lambda$, we can calculate

$$
\left|Y_{\lambda}(c)\right|=e^{\int_{0}^{c}-\operatorname{Im}(B) d x}=e^{\int_{0}^{c}(x+1)^{-1} d x}=e^{\log (c+1)-0}=c+1
$$

This shows that $Y_{\lambda} \in L_{A}^{2}$, so this is case $\mathbf{2 . b}$.

For case 2.c let $A(x):=1$ and $B(x):=-i(x+1)$. Again case 2 is obvious with $k_{1}=1$. For $\lambda=0 \in W_{1}$, the estimation

$$
\left|Y_{\lambda}(c)\right|=e^{\int_{0}^{c}-\operatorname{Im}(B) d x}=e^{\int_{0}^{c} x+1 d x} \geq e^{c}
$$

shows $Y_{\lambda} \notin L_{A}^{2}$.

The choice $B:=0$ yields case 3 . Remark 6.4 .2 shows that both sub-cases 3.a and 3.c can occur, depending on whether $A$ is in $L^{1}(0, \infty)$ or not.

These simple examples show that $A \nsucc C_{\lambda}$ is possible and even the number of linearly independent solutions of (2.1) that are in $L_{A}^{2}$ may differ from the corresponding number for $L_{C_{\lambda}}^{2}$.

Our theory yields the estimation $W_{V} \subseteq \rho(L)$. The following shows that even equality may hold. For this we want $\frac{\operatorname{Im}(B)}{A}$ to be constant, which yields case $\mathbf{3}$, furthermore we need sub-case 3.c.

For example let $A(x):=1, B(x):=x^{2}$ on $[0, \infty)$. Because $A \notin L^{1}$, this is subcase 3.c.

We obtain $k_{-1}=k_{1}=0$. Thus $W_{-1}$ is the upper, $W_{1}$ the lower half-plane. So the upper half-plane is a subset of the resolvent set of $L_{-1}$, the lower half-plane is a subset of the 
resolvent set of $L_{1}$. Corollary 6.4 .3 yields the assertion.

A weaker assumption would be that $\frac{\operatorname{Im}(B)}{A}$ is bounded and convergent to some $\tilde{k}$. This also is case 3 and, if $A$ is not in $L^{1}(0, \infty)$, sub-case 3.c.

Let $\tilde{a}>0$. We want to apply Theorem 5.4.1. For this we set

$$
\begin{aligned}
& \widetilde{k}_{-1}^{\tilde{a}}:=\inf \{k \in \mathbb{R}: k A+\operatorname{Im}(B) \geq 0 \text { a.e. on }[\tilde{a}, \infty)\} \text { and } \\
& \widetilde{k}_{1}^{\tilde{a}}:=\sup \{k \in \mathbb{R}:-k A-\operatorname{Im}(B) \geq 0 \text { a.e. on }[\tilde{a}, \infty)\} .
\end{aligned}
$$

As for the original problem we conclude

$\widetilde{W}_{-1}^{\tilde{a}}=\left\{\lambda \in \mathbb{C}: \operatorname{Im}(\lambda)>\widetilde{k}_{-1}^{\tilde{a}}\right\}$ and $\widetilde{W}_{1}^{\tilde{a}}=\left\{\lambda \in \mathbb{C}: \operatorname{Im}(\lambda)<\widetilde{k}_{1}^{\tilde{a}}\right\}$.

The assumption on $\frac{\operatorname{Im}(B)}{A}$ yields that $\widetilde{k}_{1}^{\tilde{a}}$ and $\widetilde{k}_{-1}^{\tilde{a}}$ converge to $\tilde{k}$ (as $\tilde{a}$ tends to infinity).

Recall $\widehat{b}=\infty$. Theorem 5.4.1 (respectively Corollary 5.4.2) yields that there are at most isolated points of the spectrum of $L_{-1}$ in $\{\lambda \in \mathbb{C}: \operatorname{Im}(\lambda)>\tilde{k}\}$ and there are at most isolated points of the spectrum of $L_{1}$ in $\{\lambda \in \mathbb{C}: \operatorname{Im}(\lambda)<\tilde{k}\}$.

Using Corollary 6.4.3 again, we can conclude that $\tilde{k} i$ plus the lower half-plane is in the spectrum of $L_{-1}$ and $\tilde{k} i$ plus the upper half-plane is in the spectrum of $L_{1}$.

We can even go further. If we examine the proof of Theorem 5.4.1, we realize that $\lambda$ is an isolated point in $\widetilde{W}_{V}^{\tilde{a}} \backslash \rho(L)$ only if the matrix $\Omega_{\lambda}$ in (5.28) is singular. This matrix however, independent of $V \in\{ \pm 1\}$, equals $Y_{\lambda}(\tilde{a}) \neq 0$ and thus is regular. We conclude

Corollary 6.4.4. In case 3.c, if $\frac{\operatorname{Im} B(x)}{A(x)}$ converges to some $\tilde{k}($ as $x \rightarrow \infty)$, then

$$
\begin{aligned}
\rho\left(L_{-1}\right) & =\{\lambda \in \mathbb{C}: \operatorname{Im}(\lambda)>\tilde{k}\} \quad \text { and } \\
\rho\left(L_{1}\right) & =\{\lambda \in \mathbb{C}: \operatorname{Im}(\lambda)<\tilde{k}\} .
\end{aligned}
$$

We can use Theorem 5.4.1 and the above considerations (that there are no isolated points in $\left.\widetilde{W_{V}^{\tilde{a}}} \backslash \rho(L)\right)$, to conclude generally in case $\mathbf{1}$ or $\mathbf{3}$ :

$$
\rho\left(L_{-1}\right) \supset \bigcup_{\tilde{a} \in[0, \infty)} \widetilde{W}_{-1}^{\tilde{a}}
$$

and in case $\mathbf{2}$ or $\mathbf{3}$ :

$$
\rho\left(L_{1}\right) \supset \bigcup_{\tilde{a} \in[0, \infty)} \widetilde{W}_{1}^{\tilde{a}}
$$

Recall the shape of $\widetilde{W}_{-1}^{\tilde{a}}$ and the definition of $\widetilde{k}_{-1}^{\tilde{a}}$ above (respectively of $\widetilde{W}_{1}^{\tilde{a}}$ and $\widetilde{k}_{1}^{\tilde{a}}$ ). Thus if $A$ and $B$ are such, that $\frac{\operatorname{Im}(B)}{A}$ converges to plus or minus infinity, the corresponding 
operator has empty spectrum. An example would be $A=1$ and $B(t)=-i t$ on $[0, \infty)$. This is case 1. The domain of $L_{-1}$ is given in (6.29) with $L y:=-i y^{\prime}-B y$ for $y \in D\left(L_{-1}\right)$.

The following consideration is independent of the case $(\mathbf{1}, \mathbf{2}$ or $\mathbf{3})$ :

If both problems $\left((6.20)\right.$ and (6.25)) are in the weak limit-circle case, (this means $Y_{\lambda}$ and $Z_{\lambda}$ are in $L_{A}^{2}$ for some $\lambda \in W_{1}$, respectively $\left.\lambda \in W_{-1}\right)$, then Theorem 5.3.3 and the extraordinary $M$ function (with no entries) yield that the spectrum of the corresponding operator $\left(L_{1}\right.$, respectively $L_{-1}$ ) is empty. 


\section{Table of symbols}

GENERAL FUNCTIONS, SETS AND RELATIONS

$\operatorname{codiag}\left(z_{1}, \ldots, z_{j}\right):=\left(\begin{array}{cc} & z_{j} \\ z_{1} & \end{array}\right)$

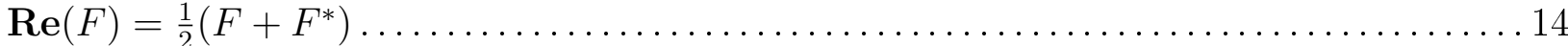

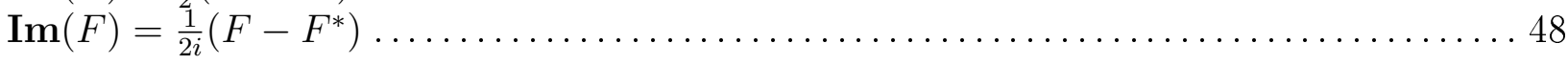

ess $\operatorname{supp}(f)=\bigcap\left\{{\overline{\left\{x \in D(f) \backslash N_{0}: f(x) \neq 0\right\}}}^{D(f)}: N_{0}\right.$ has measure 0$\} \ldots \ldots \ldots \ldots . . .87$

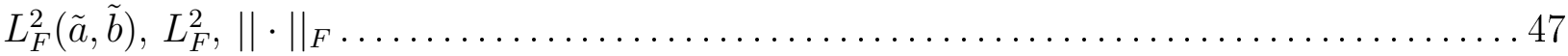

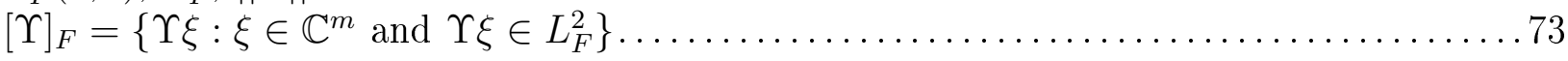

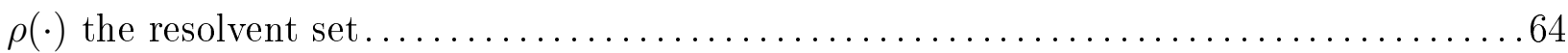

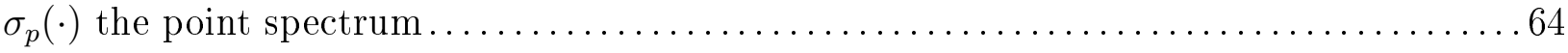

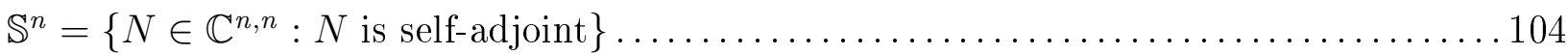

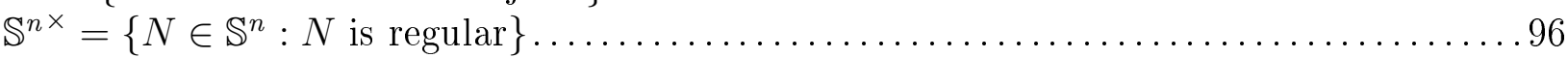

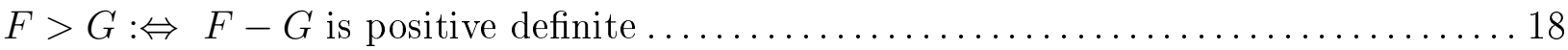

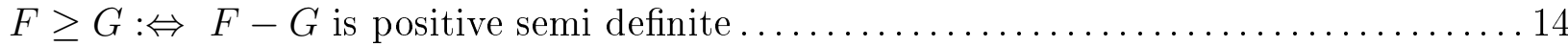

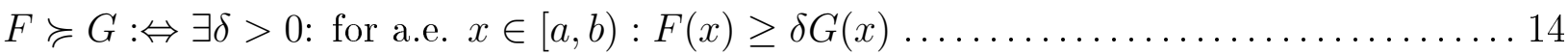

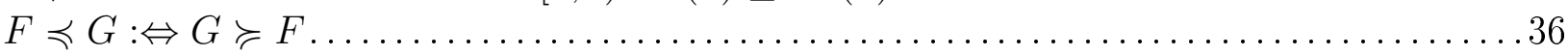

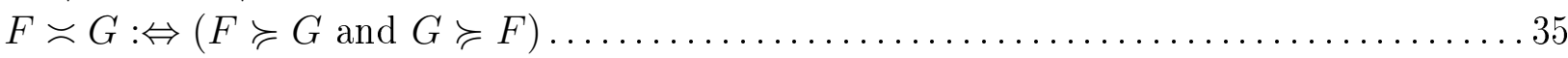

\section{RELATIONS OF THIS THESIS}

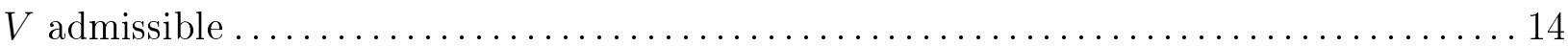

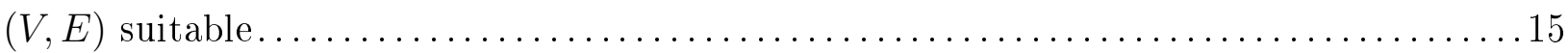


FUNCTIONS AND CONSTANTS

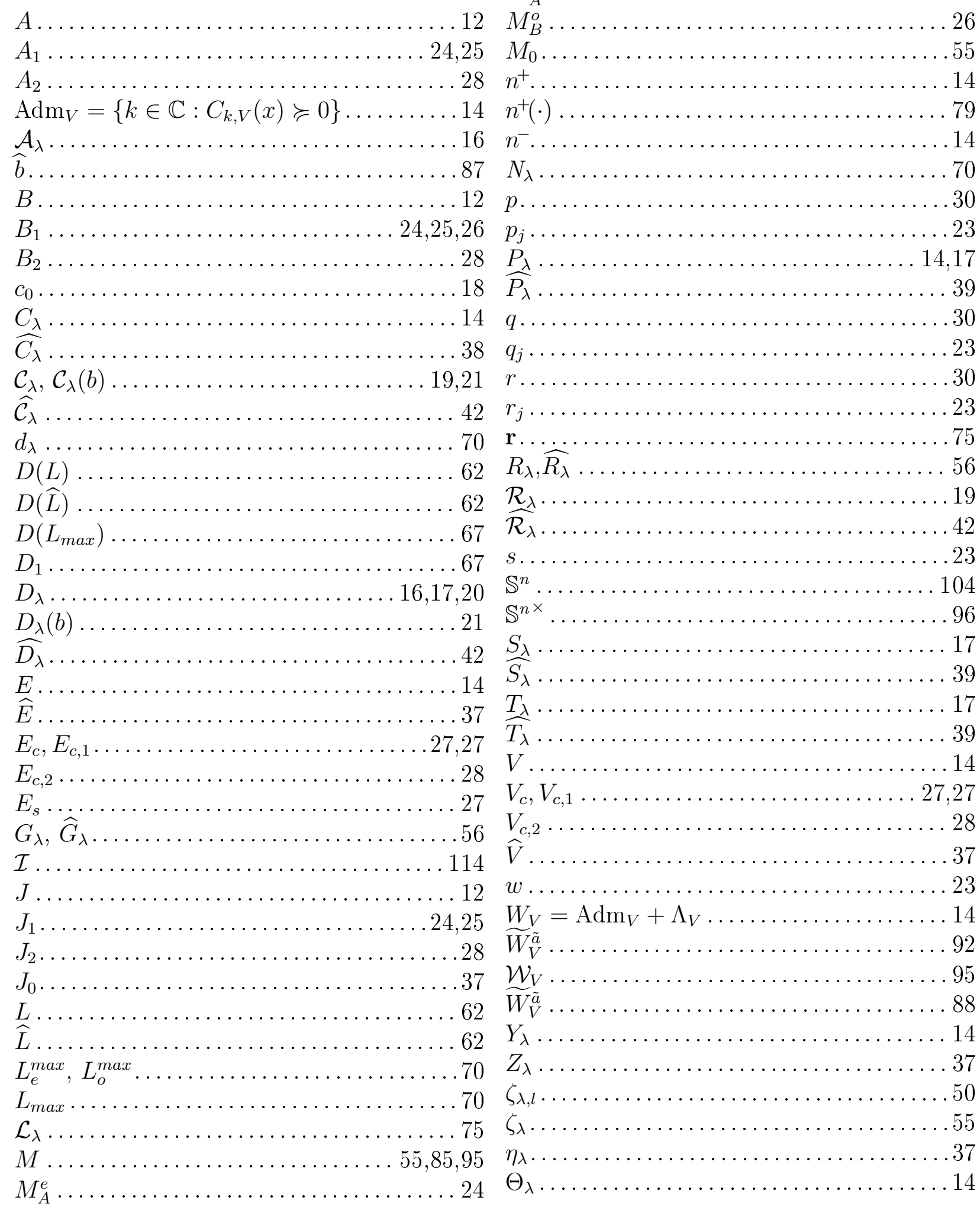




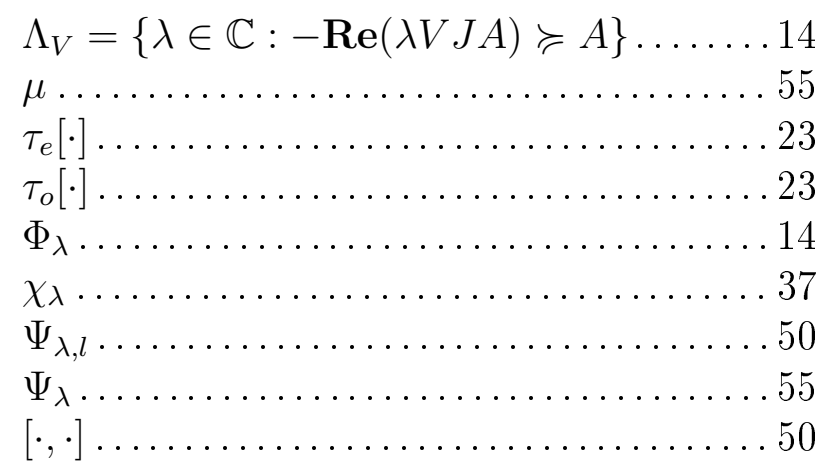

\section{CASES}

strict limit-point case . .............. 79

limit-point case .................. 78

$(\mathbf{r}$-)non-limit-point case $\ldots \ldots \ldots \ldots \ldots .78$

weak limit-circle case.................. 79

limit-circle case ................... 78

full limit-circle case ................ 78 


\section{Bibliography}

[1] F.V. Atkinson. Discrete and continuos boundary value problems. Akademic Press, New York, 1964.

[2] H. Bart. Meromorphic operator valued functions. Mathematisch Centrum Amsterdam, 1973.

[3] H. Behnke, D. Hinton. Transformation theory of symmetric differential expressions. Advances in Differential Equations, Volume 11, Number 6 (2006), 601-626.

[4] Bourbaki. Algebra I. Springer Verlag, Berlin, 1989.

[5] B.M. Brown, W.D. Evans and M. Plum. Titchmarsh-Sims-Weyl theory for complex hamiltonian systems. Proc. London Math. Soc., London 2003.

[6] B.M. Brown, D.K.R. McCormack, W.D. Evans and M. Plum. On the spectrum of secondorder differential operators with complex coefficients. Proc. Royal Society London, 1999.

[7] Earl A. Coddington and Norman Levinson. Theory of ordinary differential equations. McGraw-Hill, 1955.

[8] N. Dunford, J.T. Schwartz. Linear operators, Part II. Interscience, New York, 1963.

[9] W.N. Everitt. Integral-square, analytic solutions of odd-order, formally symmetric differential equations. Proc. London Math. Soc. (3) 25 (1972) 156-182.

[10] W.N. Everitt. Singular differential equations II: some even-order cases. Quart. J. Math. Oxford (2) 18 (1976) 13-32.

[11] W.N. Everitt, K. Kumar. On the Titchmarsh-Weyl theory of ordinary symmetric differential expressions I. The general theory. Nieuw Arch. Wisk. (3) 24 (1976) 109-145.

[12] D. Hinton, A. Schneider. Titchmarsh-Weyl coefficients for odd-order linear hamiltonian systems. Journal of Spectral Mathematics and its Applications, Volume 1, 2005.

[13] D.B. Hinton, J.K. Shaw. On Titchmarsh-Weyl $M(\lambda)$-functions for Linear Hamiltonian systems. J. Differential Eqs. 40 (1981) 316-342 
[14] D.B. Hinton, J.K. Shaw. On boundary value problems for Hamiltonian systems with two singular endpoints. SIAM J. Math. Anal. 15 (1984) 272-286

[15] T. Kato. Pertubation theory for linear operators, 2nd edn. Springer, Heidelberg, 1980.

[16] A.M. Krall. $M(\lambda)$ theory for singular Hamiltonian systems with one singular endpoint. Siam J. Math. Anal. 20 (1989), 664-700.

[17] M.A. Naimark. Linear differential operators. Frederik Ungar Publishing Co., New York, 1968.

[18] M.A. Naimark. Linear differential operators II. Frederik Ungar Publishing Co., New York, 1968.

[19] A.R. Sims. Secondary conditions for linear differential operators of the second order. J. Mathematics and Mechanics Ann. Mat. Pure 6 (1957), 247-285.

[20] E.C. Titchmarsh. Eigenfunction expensions associated with second-order differential equations. Claredon Press, Oxford, 1946.

[21] P.W. Walker. A vector-matrix formulation for formally symmetric ordinary differential equations with applications to solutions of integrable-square. J. London Math. Soc. (2) 9 (1974) 151-159.

[22] W. Walter. Ordinary differential equations. Springer Verlag, New York, 1998.

[23] H. Weyl. Über gewöhnliche Differentialgleichungen mit Singularitäten und die zugehörigen Entwicklungen. Math. Ann. 68 (1910), 220-269.

[24] A. Zettl. Formally self-adjoint quasi-differential operators. Rocky Mountain J. Math. 5 (1975) 453-474. 